\title{
Mechanisms of pronuclear migration in mammalian zygotes
}

\author{
Dissertation \\ for the award of the degree \\ "Doctor rerum naturalium" (Dr.rer.nat.) \\ of the Georg-August-Universität Göttingen \\ within the doctoral program \\ Molecular Biology of Cells
}

of the Göttingen Graduate Center for Neurosciences, Biophysics, and Molecular Biosciences (GGNB)

in the Georg-August University School of Science (GAUSS)

Submitted by

Julia Uraji (née Franke)

born in Bad Homburg v.d. Höhe, 9. February 1993

Göttingen, July 2019 


\section{Thesis Committee}

Dr. Melina Schuh, Department of Meiosis, Max-Planck-Institute for Biophysical Chemistry

Prof. Dr. Sigrid Hoyer-Fender, Johann-Friedrich-Blumenbach-Institute of Zoology and AnthropologyDevelopmental Biology, Göttingen Center for Molecular Biosciences.

PD Dr. Roland Dosch, Institute of Human Genetics, University Medical Center Göttingen

\section{Members of the Examination Board}

First Reviewer: Dr. Melina Schuh, Department of Meiosis, Max-Planck-Institute for Biophysical Chemistry

Second Reviewer: Prof. Dr. Sigrid Hoyer-Fender, Johann-Friedrich-Blumenbach-Institute of Zoology and Anthropology-Developmental Biology, Göttingen Center for Molecular Biosciences.

\section{Further members of the Examination Board:}

PD Dr. Roland Dosch, Institute of Human Genetics, University Medical Center Göttingen

Dr. Dieter Klopfenstein, Department of Biophysics, Third Institute of Physics, Georg-August-Universität Göttingen

Dr. Peter Lenart, Research Group Cytoskeletal Dynamics in Oocytes \& Live-cell Imaging Facility, MaxPlanck-Institute for Biophysical Chemistry

Dr. Ufuk Günesdogan, Department of Developmental Biology, Göttingen Center for Molecular Biosciences, Georg-August-Universität Göttingen

Date of the oral examination: $22^{\text {nd }}$ August 2019 


\section{TABLE OF CONTENTS}

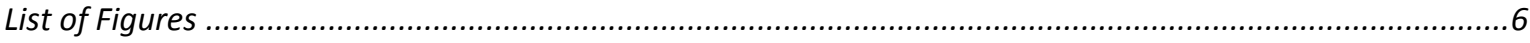

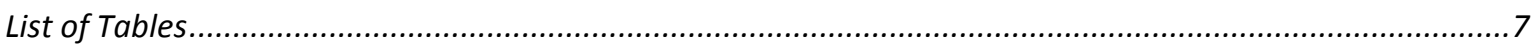

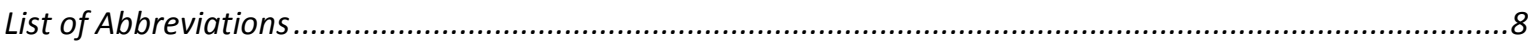

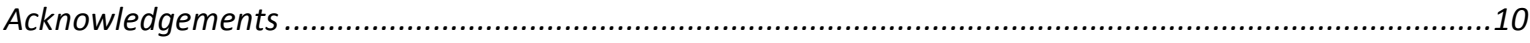

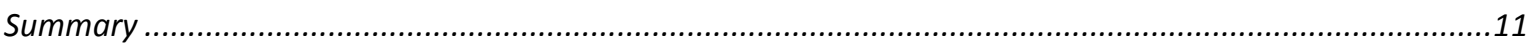

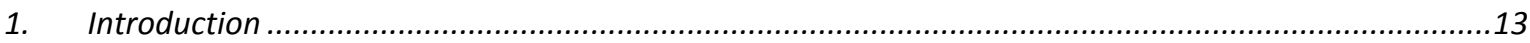

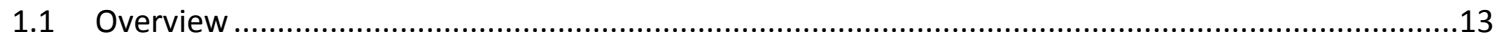

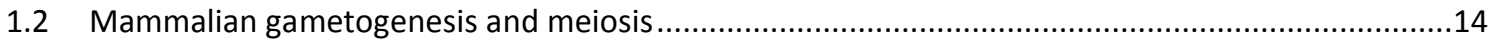

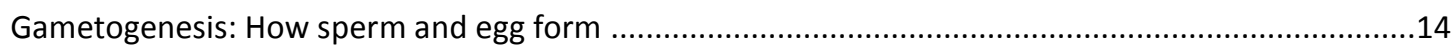

Mammalian spermatogenesis and male meiosis.......................................................................14

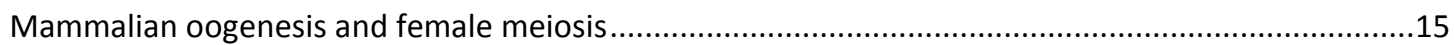

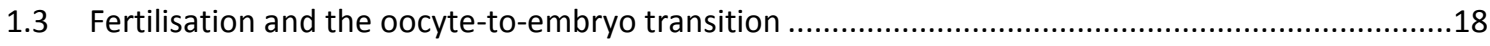

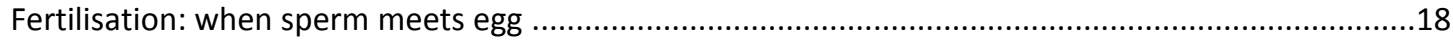

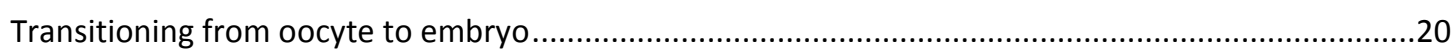

1.4 The mechanisms of (pro)nuclear migration in different contexts ................................................22

The role of microtubules and centrosomes in nuclear migration .................................................23

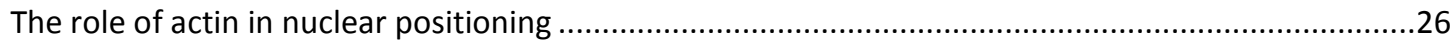

Nuclear migration without a centrosome: mouse oocytes and zygotes .......................................29

A novel set of tools to investigate pronuclear migration in mouse zygotes........................................31

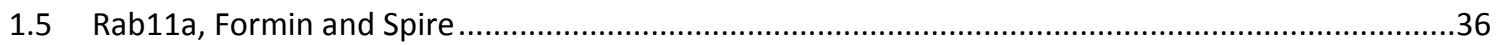

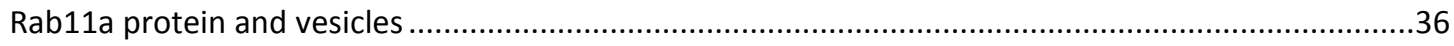

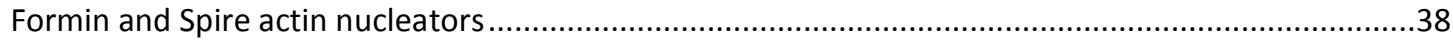

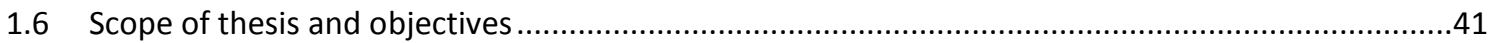

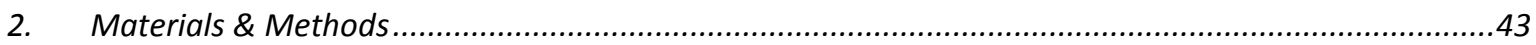

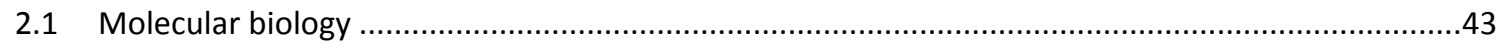

Cloning of new expression constructs for live imaging in mouse zygotes.........................................43

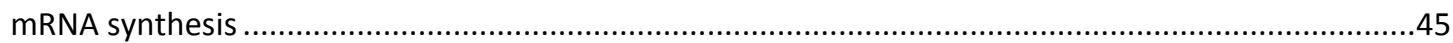

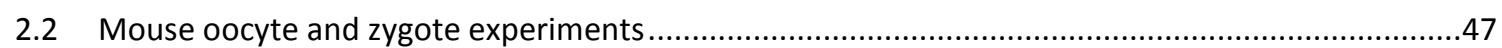

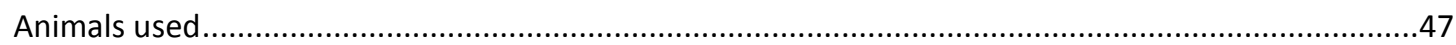

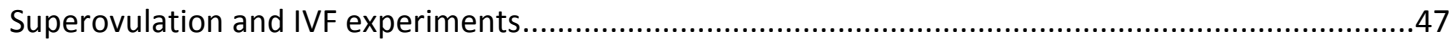

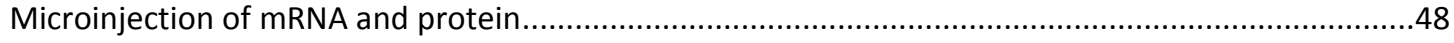

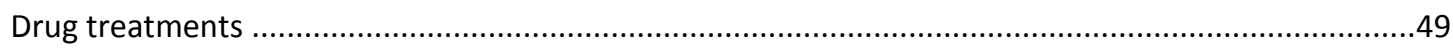

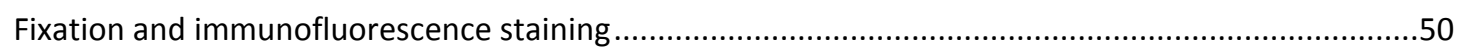

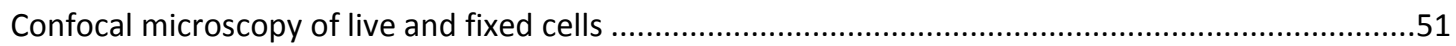




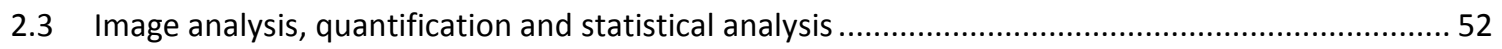

3D-tracking and quantitative analysis of pronuclear migration ................................................... 52

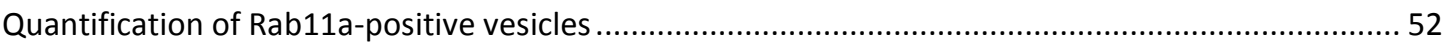

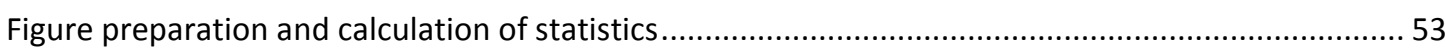

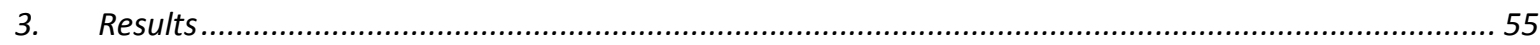

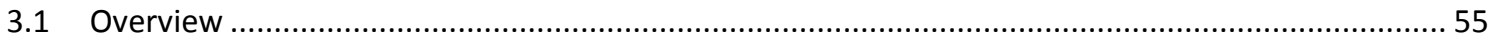

3.2 Rab11a is required for the early, fast migration of the male pronucleus ................................... 55

3.3 Rab11a-positive vesicles are present in the early, but not the late, mouse zygote........................60

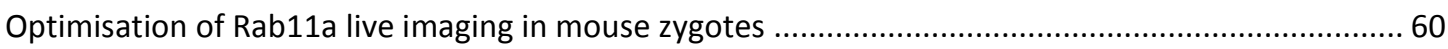

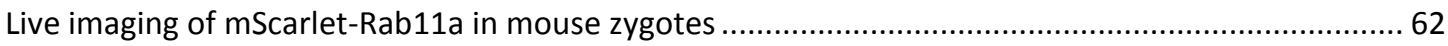

Testing different antibodies for Rab11a and related Spire and Formin actin nucleators.................... 65

Optimising Rab11a immunofluorescence staining and imaging conditions in mouse zygotes .............68

Confirming co-localisation of Rab11a antibody staining and Rab11a-GFP in fixed zygotes ................. 71

Immunofluorescence staining of Rab11a in zygotes at different time points after insemination ........ 74

Rab11a-positive vesicles are absent in MII oocytes and zygotes with perturbed Rab11a function ...... 78

3.4 Rab11a and actin nucleators accumulate adjacent to the forming male pronucleus ................... 83

Live imaging of mScarlet-Rab11a in high temporospatial resolution at the forming pronuclei .............83

Live imaging of mScarlet-Rab11a at the male pronucleus with Rab11a perturbations........................ 88

Optimisation of Spire2 and Formin-2 live imaging in the mouse zygote ......................................... 90

Co-expression of Rab11a and Spire2 at the male pronucleus .................................................... 93

Live imaging of mClover3-Spire2 in high temporospatial resolution at the forming pronuclei............ 95

3.5 The functional role of actin nucleation on the fast migration of the male pronucleus ................... 99

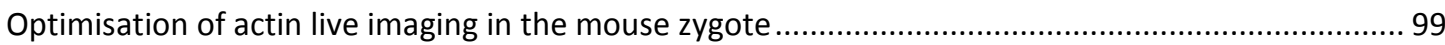

Spire2-Formin-2 interaction is required for fast migration of the male pronucleus ........................ 103

mScarlet-Rab11a and mClover3-Spire2 accumulation by the male pronucleus require Spire2-Formin-2

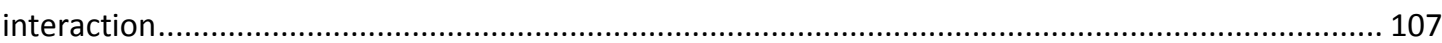

Overexpression of Spire2 elongates fast migration of pronuclei................................................. 110

3.6 Fast migration of pronuclei and enrichment of Spire2, Rab11a are not dependent on sperm entry site, nor the fertilisation cone

The female pronucleus exhibits fast migration and mScarlet-Rab11a, mClover3-Spire2 accumulation when it forms close to the cortex

Fast pronuclear migration is possible without actomyosin contractions of the fertilisation cone...... 119

Spire2 accumulation at the forming male pronucleus persists when actomyosin contractions are

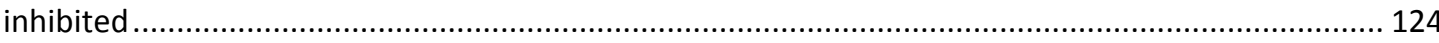

3.7 Actin is absolutely essential for pronuclear migration in later pronuclear migration .................. 128

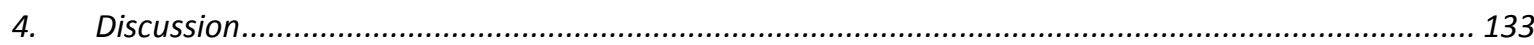

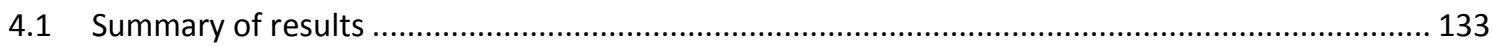


Rab11a, Spire2 and Formin-2 are present at and required for the fast, early pronuclear migration away from the cortex

Fast pronuclear migration away from the cortex is not dependent on sperm entry site or the fertilisation cone

Actin is absolutely essential for early and late pronuclear migration

4.2 A new model for pronuclear migration in mouse zygotes ......................................................138

4.3 Open questions \& future experiments to test the model ......................................................143

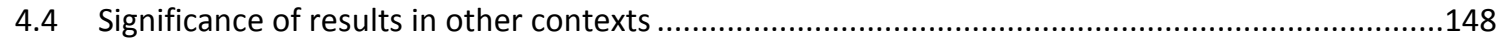

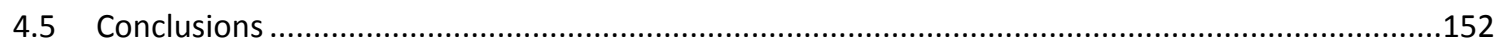

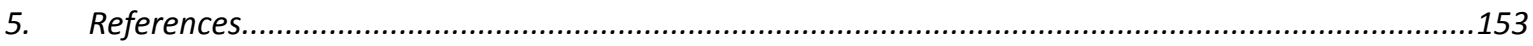

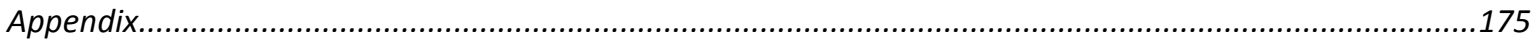




\section{LIST OF FIGURES}

Figure 1: Overview of mammalian pre-implantation development and oocyte maturation........................... 17

Figure 2: The role of microtubules, actin and intermediate filaments in nuclear positioning..........................23

Figure 3: 3D-tracking pronuclear migration in mouse zygotes over time. .................................................. 32

Figure 4: brefeldin A treatment perturbs the early, fast migration of the male pronucleus. ............................. 34

Figure 5: Rab11a is required for the early, fast migration of the male pronucleus. .......................................58

Figure 6: Optimisation of mScarlet-Rab11a mRNA concentration and imaging conditions for live-cell imaging of

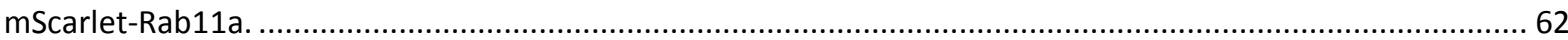

Figure 7: Live imaging of mScarlet-Rab11a from fertilisation to the first mitotic division..................................65

Figure 8: Antibody testing of Formin-2, Spire1/2, and Rab11a antibodies in GV oocytes..............................68

Figure 9: Optimisation of Rab11a immunofluorescence staining and imaging conditions.............................. 70

Figure 10: Co-localisation of EGFP-Rab11a and Rab11a immunofluorescence staining................................. 73

Figure 11: Immunofluorescence staining and quantification of Rab11a-positive vesicles in zygotes at different time points after insemination. 78

Figure 12: Immunofluorescence staining of MII oocytes, brefeldin A-treated and SNAP-Rab11a ${ }^{\mathrm{S} 25 \mathrm{~N}}$-expressing mouse zygotes.

Figure 13: Live imaging of mScarlet-Rab11a in high temporospatial resolution at male and female pronuclei. 87

Figure 14: Live imaging of mScarlet-Rab11a at the male pronucleus with Rab11a perturbations. 89

Figure 15: Optimisation of mEGFP-Spire2, mCherry-Spire2, and Formin-2-EGFP live imaging in mouse zygotes. 92

Figure 16: Co-expression of mScarlet-Rab11a and EGFP-Spire2 at the male pronucleus. .95

Figure 17: Live imaging of mClover3-Spire2 in high temporospatial resolution at male and female pronuclei. . 97

Figure 18: Optimisation of different actin probes for live imaging in mouse zygotes. ................................... 102

Figure 19: Spire2-Formin-2 interaction is required for fast migration of the male pronucleus. 106

Figure 20: mScarlet-Rab11a and mClover3-Spire2 accumulation by the male pronucleus require Spire2-Formin2 interaction 110

Figure 21: Overexpression of mEGFP-Spire2 elongates fast migration of pronuclei. 114

Figure 22: The female pronucleus exhibits fast migration and mScarlet-Rab11a, mClover3-Spire2 accumulation when it forms close to the cortex. 119

Figure 23: Fast pronuclear migration is possible without actomyosin contractions of the fertilisation cone... 124

Figure 24: mClover3-Spire2 accumulation persists when actomyosin contractions are inhibited with ML-7 ... 127

Figure 25: Late pronuclear migration requires F-actin......................................................................... 130

Figure 26: A new model for pronuclear migration in mouse zygotes. ........................................................ 142

Figure 27: Cytochalasin D treatment completely perturbs pronuclear migration in mouse zygotes. ............... 175

Figure 28: Nocodazole treatment can make the female pronucleus form closer to the cortex.................... 176

Figure 29: Co-expression of mScarlet-Spire2 and Formin-2-EGFP mRNA. ........................................... 177

Figure 30: Fast phase of male pronuclear migration is elongated by SNAP-Spire2 Overexpression................ 177 


\section{LIST OF TABLES}

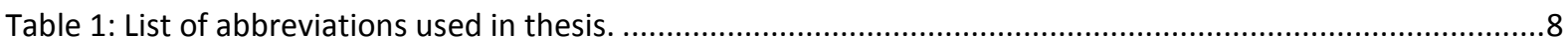

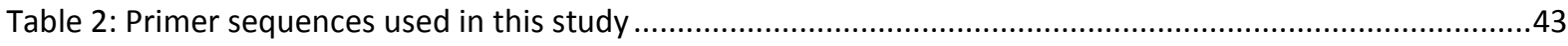

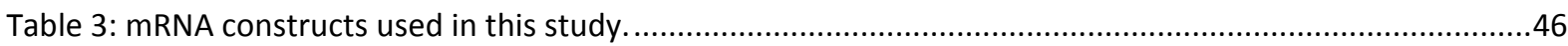

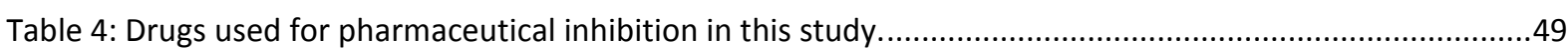

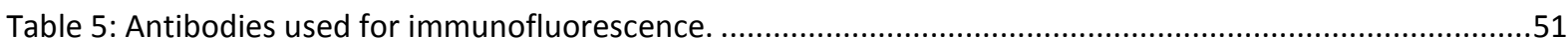




\section{LIST OF ABBREVIATIONS}

Table 1: List of abbreviations used in thesis. Note that due to varying nomenclature between genes and protein, as well as different species, names are listed in the nomenclature of the protein as used in the relevant literature, unless otherwise stated.

\begin{tabular}{|c|c|}
\hline Abbreviation & Full name \\
\hline (db)cAMP & (Dibutyryl) Cyclic adenosine monophosphate \\
\hline (ds)DNA & (double-stranded) Deoxyribonucleic acid \\
\hline$(m)$ (E)GFP & (mouse) (Enhanced) Green fluorescent protein \\
\hline (m)RNA & (messenger) Ribonucleic acid \\
\hline ADF & Actin-depolymerizing factor / Cofilin \\
\hline APC & Adenomatous Polyposis Coli \\
\hline $\mathrm{APC} / \mathrm{C}$ & Anaphase-promoting complex/cyclosome \\
\hline ARF(1p) & ADP ribosylation factor (1 protein) \\
\hline ARP2/3 & Actin-related protein $2 / 3$ \\
\hline ATP & Adenosine triphosphate \\
\hline BMP & Bone morphogenetic protein \\
\hline BRG1 & Brahma-related gene 1 \\
\hline $\mathrm{Ca}^{2+}$ & Calcium ions \\
\hline CaMKII & $\mathrm{Ca}^{2+} / \mathrm{calmodulin}$-dependent protein kinase II \\
\hline CD9 & Cluster of differentiation protein 9 \\
\hline CDC42 & Cell division control protein 42 homolog \\
\hline CSF & Cytostatic factor \\
\hline DISC1 & Disrupted in schizophrenia 1 protein \\
\hline DYRB-1 & Dynein light chain roadblock 1 \\
\hline EMI2 & Early mitotic inhibitor 2 \\
\hline $\mathrm{FH}(1 / 2)$ & Formin homology domain (1/2) \\
\hline FHOD1 & Formin homology domain-containing protein 1 \\
\hline Fmn2 & Formin-2 (gene) \\
\hline GAP & GTPase-activating/accelerating proteins \\
\hline GDF & GDI displacement factor \\
\hline GDI & Guanosine nucleotide dissociation inhibitor \\
\hline GDP & Guanosine diphosphate \\
\hline GEF & Guanine nucleotide exchange factor \\
\hline GTP(ase) & Guanosine triphosphate (-ase) \\
\hline GV & Germinal vesicle \\
\hline hpi & Hours post insemination \\
\hline ICSI & Intra-cytoplasmic sperm injection \\
\hline $\mathrm{IP}_{3}$ & Inositol $(1,4,5-)$ trisphosphate \\
\hline IVF & In vitro Fertilisation \\
\hline KASH & Klarsicht, ANC-1, Syne homology \\
\hline KIND & Kinase non-catalytic C-lobe domain \\
\hline $\mathrm{LH}$ & Luteinising hormone \\
\hline LINC & Linker of Nucleoskeleton and Cytoskeleton \\
\hline LIS-1 & Lissencephaly-1 \\
\hline $\mathrm{M} / \mathrm{GO} / 1 / 2 / \mathrm{S}$ & Stage of cell cycle - Mitosis, Gap 0/1/2, Synthesis phases \\
\hline MAPK & Mitogen-activated protein kinases \\
\hline mDia (1/3) & Mouse Diaphanous homolog (1/3) \\
\hline $\mathrm{Ml} / \mathrm{MII}$ & Meiosis I/II \\
\hline ML-7 & Selective inhibitor of myosin light chain kinase \\
\hline MLCK & Myosin light-chain kinase \\
\hline MRCK & Myotonic dystrophy kinase-related Cdc42 binding kinase \\
\hline MTOC & Microtubule organizing centre \\
\hline
\end{tabular}




\begin{tabular}{|l|l|}
\hline NEBD (in figures) & Nuclear Envelope Breakdown \\
\hline PCR & Polymerase Chain Reaction \\
\hline PGC (7) & Primordial germ cell (protein 7, aka Dppa3/Stella) \\
\hline PLC & Phospholipase C zeta \\
\hline PLK1 & Serine/threonine-protein / polo-like kinase 1 \\
\hline PN (in figures) & Pronucleus \\
\hline Rab (7/11a/11b/25) & Ras-related protein (7/11a/11b/25) \\
\hline RAD9A & RAD9 checkpoint clamp component A \\
\hline Ran & Ras-related Nuclear protein \\
\hline RILP & Rab-interacting lysosomal protein \\
\hline SAMP1 & Spindle associated membrane protein 1 / transmembrane protein 201 \\
\hline Sec7 & Secretory ARF-family GEFs \\
\hline SNARE & Soluble N-ethylmaleimide-sensitive factor activating protein (SNAP) Receptor \\
\hline Sry & Sex-determining region Y \\
\hline SUN (-1/2) & Sad1 an UNC-84 (protein 1/2) \\
\hline TAN & Transmembrane actin-associated nuclear lines \\
\hline TET3 & Ten-eleven translocation methylcytosine dioxygenase 3 \\
\hline UNC-83 & Un-coordinated nuclear migration protein -83 \\
\hline WH2 & Wiskott-Aldrich homology 2 \\
\hline ZP $(1 / 2 / 3 / 4)$ & Zona pellucida (protein 1/2/3/4) \\
\hline ZYG-12 & Zygote defective protein 12 \\
\hline & \\
\hline
\end{tabular}




\section{ACKNOWLEDGEMENTS}

I would firstly like to thank my group leader, Melina Schuh, for giving me the opportunity to carry out my doctoral studies in the department of Meiosis at the Max-Planck Institute for Biophysical Chemistry, and to spend the last months at the Bourn Hall Clinic in England.

Secondly, I would like to thank my project supervisor and collaborator Kathleen Scheffler for her guidance and team-work. Before I joined this project, you established the system and made observations without which my contributions would not have been possible. I have learned a lot, about both the scientific and personal, from our teamwork and discussions.

Further thanks are owed to the entire department of Meiosis for a productive work environment and fruitful discussions, as well fantastic and frequent birthday cakes. In particular, I would like to thank Ann-Sophie, Kata, Kathleen, Ina, Ida and Vera for always lending an ear and being great friends. I would also like to thank our technicians Tina, Silke and Lydia, as well as our lab manager Lena, without whom our lab could not function. Lena, thank you also for your support while I've been in Bourn, and for offering your time to proof-read this thesis. Further thanks for proof-reading are owed to Kay, Andrea and Kata.

I would like to thank all of the staff at the Bourn Hall Clinic, in particular the embryology team, the nurses, admin staff, Donna and Andrea next-door, and of course the patients, as well as the Norwich clinical team. A huge thanks is owed to Bianka Seres, who established this satellite lab during her PhD time, and who taught me so much both here and back in Germany. Also, I would like to thank Kay Elder and Martyn Blaney for making this research possible; Kay, thank you for your advice and guidance during my time in Bourn.

I am grateful to my second and third thesis advisory committee members, Sigrid Hoyer-Fender and Roland Dosch, for the supervision and useful advice during our meetings throughout the years. I would also like to thank the other members of my defence committee, Dieter Klopfenstein, Peter Lenart and Ufuk Günesdogan.

This project was funded by the Max Planck Society, and other projects I was part of were funded by ERC grant \#337415 as well as the Lister Research Prize.

Outside of the scientific bubble, I would also like to thank my family and close friends for the longdistance support. Alex - thank you for the never-ending support, for taking my mind off work on the weekends, and recharging my 9V Block Batterie. Lastly, I would like to give myself a pat on the back the last three years weren't easy, but I stuck through. 


\section{SUMMARY}

In sexually reproducing species, life begins with the fusion of egg and sperm to form the one-cell embryo, called the zygote. Maternal and paternal DNA form the female and male pronuclei, and must migrate to each other to allow the unison of genetic information and the first mitotic division. This pronuclear migration relies on microtubules and microtubule-related motors in many studied systems. However, canonical centrosomes are absent in the mouse zygote. Interestingly, studies suggest that actin is essential for pronuclear migration in this centrosome-free system (Chaigne et al., 2016; Maro et al., 1984). However, the precise temporospatial dynamics of pronuclear migration, as well as the role of specific cytoskeletal players, remained unknown. A postdoctoral scientist in our laboratory, Kathleen Scheffler, established a protocol to culture and image mouse zygotes throughout pronuclear migration. Using this system, she discovered that the male pronucleus forms closer to the cortex than the female pronucleus, and shows a fast inward migration in the first hour after pronuclear formation. Her preliminary results indicated that actin vesicles may be important for this fast migration, and I joined the project to investigate the mechanisms of this first fast pronuclear migration in mouse zygotes, with a focus on Rab11a vesicles and related actin nucleators. This was achieved using a combination of observational and functional experimental approaches. Perturbation of Rab11a inhibited the fast migration of the male pronucleus. Live and fixed cell imaging was optimised and used to describe the localisation of Rab11a throughout zygotic development and pronuclear migration, revealing that large Rab11a vesicles appear after fertilisation, and disappear soon after pronuclear formation. Next, high temporospatial imaging of mScarlet-Rab11a revealed an accumulation adjacent to the male pronucleus during its formation. A similar accumulation of actin nucleators Spire2 and Formin-2 was also found using live imaging. Inhibition and overexpression approaches confirmed a functional role of these proteins in the fast migration of the male pronucleus away from the cortex; inhibition of the Formin-2/Spire2 interaction with $\mathrm{FH} 2$ resulted in a slower initial migration, and overexpression of Spire2 elongated the fast migration phase. Interestingly, when the maternal DNA was induced to form a pronucleus close to the cortex, accumulations of mScarletRab11a and mClover3-Spire2 were also observed by the forming female pronucleus. Furthermore, when actomyosin contractions of the fertilisation cone were inhibited with ML-7, fast pronuclear migration was still possible. Lastly, addition of cytochalasin D after pronuclear formation inhibited any further migration towards the centre of the zygote, indicating F-actin to be important for later pronuclear migration through other mechanisms than Rab11a and Spire. Overall, this study was able to implicate a functional role of Rab11a and related actin nucleators in early pronuclear migration in mouse zygotes, contributing to our understanding of pronuclear migration mechanisms in the absence of canonical centrosomes. 
This page is intentionally left blank 


\section{INTRODUCTION}

\subsection{Overview}

I had the privilege of working on several different projects during my doctoral studies. In the first year, I investigated the role of the protein Pcm-1 in spindle assembly of mouse oocytes; however, due to loss of specificity of a commercial antibody, this project could not be continued, and I used the results to write my Master Equivalence Thesis. I then began working with Kathleen Scheffler on the project which will be the focus of this thesis: investigating pronuclear migration in mouse zygotes. The experiments targeted for publication were completed by October 2018 , and I then spent the rest of my doctoral studies at our satellite laboratory in Bourn Hall Clinic, England, where I managed the lab independently, continued to analyse data and write this thesis, and conducted experiments on human oocytes for a variety of projects in collaboration with other lab members back in Göttingen. The human oocyte projects are not yet close to completion, and my thesis is therefore focused on the project that occupied the majority of my time, and feel I contributed to the most.

The goal of my project has been to understand the mechanisms of pronuclear migration in mouse zygotes, a fundamental process of the early embryo. In this introduction, I will first set the scene by giving a brief background of sperm and egg development in mammals, with a focus on findings from the mouse model system. The next section will explore fertilisation, and the changes that occur in the zygote to allow early embryonic development. Pronuclear migration is a crucial process that must take place after fertilisation to allow the first mitotic division, and this is the focus of this thesis. Mechanisms of pronuclear migration have been explored in several organisms, and the findings from these will be described. However, as mouse zygotes do not appear to fit into this general model, other examples of nuclear positioning in non-zygote systems and the role of microtubules and actin will be described in more detail. As pronuclear migration in mice was not previously well understood, Kathleen Scheffler, a postdoctoral fellow in the Department of Meiosis, set out to establish a method of researching this process on a functional level. These methods and her early findings are fundamental to this project, and thus, these currently unpublished results will be shown as part of this section. When I joined the project, I studied the contributions of the actin cytoskeleton, with a focus on Rab11a vesicles and the actin nucleators Spire2 and Formin-2. Thus, the final section of the literature review will provide a background of the current knowledge about these structures and proteins, and methods to perturb them in vivo. Lastly, the biological questions and experimental objectives of this project will be clearly defined. 


\subsection{Mammalian gametogenesis and meiosis}

\section{Gametogenesis: How sperm and egg form}

The majority of eukaryotic species have offspring through symmetric sexual reproduction, which requires the fusion of haploid gametes to form a diploid, totipotent zygote (reviewed by Otto \& Lenormand, 2002). In the case of animal species, the male and female gametes are known as the sperm and egg respectively.

In mammals, both sperm and egg begin their development in the embryo, when BMP signals in the epiblast initiate differentiation of PGCs. PGCs proliferate and migrate, colonising the future gonad at around embryonic day E10.5-11.5 in the mouse embryo. From this point onwards, the PGCs are known as gonocytes in male, and oogonia in female mammals; they cluster and form germ cell nests. Both these germ cells and the surrounding somatic tissue will undergo male or female sex differentiation, to form the testis and spermatogonium, or ovary and oogonium respectively (reviewed by Larose et al. 2019). How and when these cells will form mature gametes ready for fertilisation differs between the sexes and in different species.

Importantly, at some point in their maturation, sperm and egg progenitor cells must undergo a specialised cell division termed meiosis, which in contrast to its somatic counterpart of mitosis, is a reductional process. This is because gametes must be haploid to form a diploid zygote when they fuse. To achieve this haploid state, one round of DNA replication is followed by two consecutive rounds of cell division. In meiosis I (MI), homologous chromosome pairs are segregated, followed by sister chromatid segregation in meiosis II (MII), leaving a single chromatid of each chromosome in the gamete. Due to these rounds of random segregation, which can result in many different combinations of chromosomes in the resulting gametes and embryos, meiosis is critical for greater genetic variety in sexually reproducing species. Furthermore, the meiotic process of recombination, which occurs between crossed-over homologous chromosomes in $\mathrm{Ml}$, further contributes to this potential variety. Thus, meiosis is key to ensure both genetic variety and euploidy in offspring of sexually reproducing species (reviewed by Hörandl 2013).

Even though both sperm and egg form from PGCs colonising the genital ridge and undergo meiosis, the process of spermatogenesis and oogenesis as well as the nature of their meiotic divisions differ greatly from each other; a brief overview of their development in mice will be given next.

\section{Mammalian spermatogenesis and male meiosis}

In male mouse embryos, sex determination is initiated by Sry expression at E10.5, which induces differentiation of Sertoli cells. Signals from Sertoli cells and possibly peritubular and Leydig cells induce 
PGCs to adopt a male fate in the forming testis at around E12.5-13.5 (reviewed by Spiller and Bowles, 2015). The germ cells localise close to the Sertoli cells in the seminiferous cords, soon to form the seminiferous tubules. Subsequently, the prospermatogonia arrest at G0 stage around E12.5-E14.5 (Western et al., 2008); a group of these prospermatogonia form the mitotic spermatogenic stem cell niche. In rodents, prospermatogonia remain in this GO arrest until after birth. The first round of spermatogenesis occurs shortly after birth with a specific subset of prospermatogonia developing to form spermatozoa by postnatal day $\mathrm{P} 5$, with subsequent rounds of spermatogenesis relying on the ever-dividing progenitor pool. Following this first round, spermatogenesis occurs in precisely timed stages, and cells in different stages are found spatially arranged along the length of the seminiferous tubules. Every 8.6 days, the transition from A-type spermatogonia of the progenitor pool to A1 spermatogonia occurs. Each subsequent stage also takes 8.6 days, meaning there is a well-timed 'wave' of spermatogenesis through the seminiferous epithelium. As such, after 8.6 days, the A1 spermatogonia will enter meiosis, while the next cells from the progenitor pool transition to A1 spermatogonia, and so forth. Spermatocytes undergo two rounds of meiosis to eventually form four haploid spermatids each, after a total of five transition steps, each 8.6 days apart (reviewed by Griswold, 2016). During spermiogenesis, spermatids elongate, grow tails, and undergo several other maturation steps, including replacement of histones with protamines during the condensation of the DNA. Spermatozoa are then transported to and stored in the epididymis, where they become motile and wait for ejaculation (reviewed by O'Donnell, 2014). Spermatogenesis and spermiogenesis consistently occurs throughout the life of male animals, from puberty until death.

\section{Mammalian oogenesis and female meiosis}

After PGCs have colonised the forming female gonad in the mouse embryo, they begin to enter meiosis at approximately E13.5. They arrest at the diplotene stage of prophase I between E17.5 and shortly after birth (Borum, 1961), and remain in this arrest as so-called GV oocytes until induced to continue their maturation in the post-pubescent female mammal (MacLennan et al., 2015). These GV oocytes are stored in their ovarian follicles, where they continue to grow. During this growth, the zona pellucida also forms as a glycoprotein matrix surrounding the oocyte. Towards the end of their growth, transcription comes to a halt, and only already-present mRNA is expressed from this point onwards until zygotic genome activation (Bouniol-Baly et al., 1999). The LH peak leading up to ovulation results in a decrease in CAMP in the oocyte, leading to its release from dictyate arrest (Tsafriri et al., 1996), and initiation of female meiosis. The nuclear envelope breaks down, and the first meiotic spindle begins to form. Microtubules attach to and align the homologous chromosome pairs, and the bipolar spindle migrates towards the cortex. Anaphase and cytokinesis of MI thus occurs at approximately the same time as ovulation of the egg from the follicle. In this first meiotic division extrusion, half of the 
homologous chromosomes are segregated into a very small cell called the first polar body. The oocyte then arrests in metaphase of MII, in which the second meiotic spindle has formed and aligned the remaining sister chromatids at the metaphase plate near the oocyte's cortex (reviewed by Mogessie et al., 2018). MII is completed, and the second polar body released, upon an increase in calcium signalling induced by sperm entry (Miao et al., 2012). The fertilised egg, or zygote, thus contains two haploid pronuclei, female and male, the remaining maternal chromosomes having been extruded into the polar bodies, although these will quickly degrade (Miao et al., 2004). An overview of the process of oocyte maturation and pre-implantation development is shown in Figure 1; female meiosis is shown in Figure 1B.

Thus, a key difference between male and female mammalian meiosis is that while spermatogenesis results in four functional spermatozoa from one parent cell, oogenesis produces only one oocyte per progenitor cell, with the remaining chromosomes segregated into two polar bodies through highly asymmetric cytokinesis. These asymmetric divisions are thought to ensure that maternal stores in the form of proteins, mRNAs, and metabolites in the egg are sufficient for early embryonic development, as well as to avoid fertilisation of multiple cells within one zona pellucida (reviewed by Brunet and Verlhac, 2011). Furthermore, these asymmetric divisions have been suggested to ensure spatial separation of the sperm DNA and meiotic spindle following fertilisation (Panzica and McNally, 2018). Also, as opposed to their male counterparts, female mammals have a limited reproductive lifespan, with oocyte maturation and ovulation taking place only from puberty until menopause. 
a

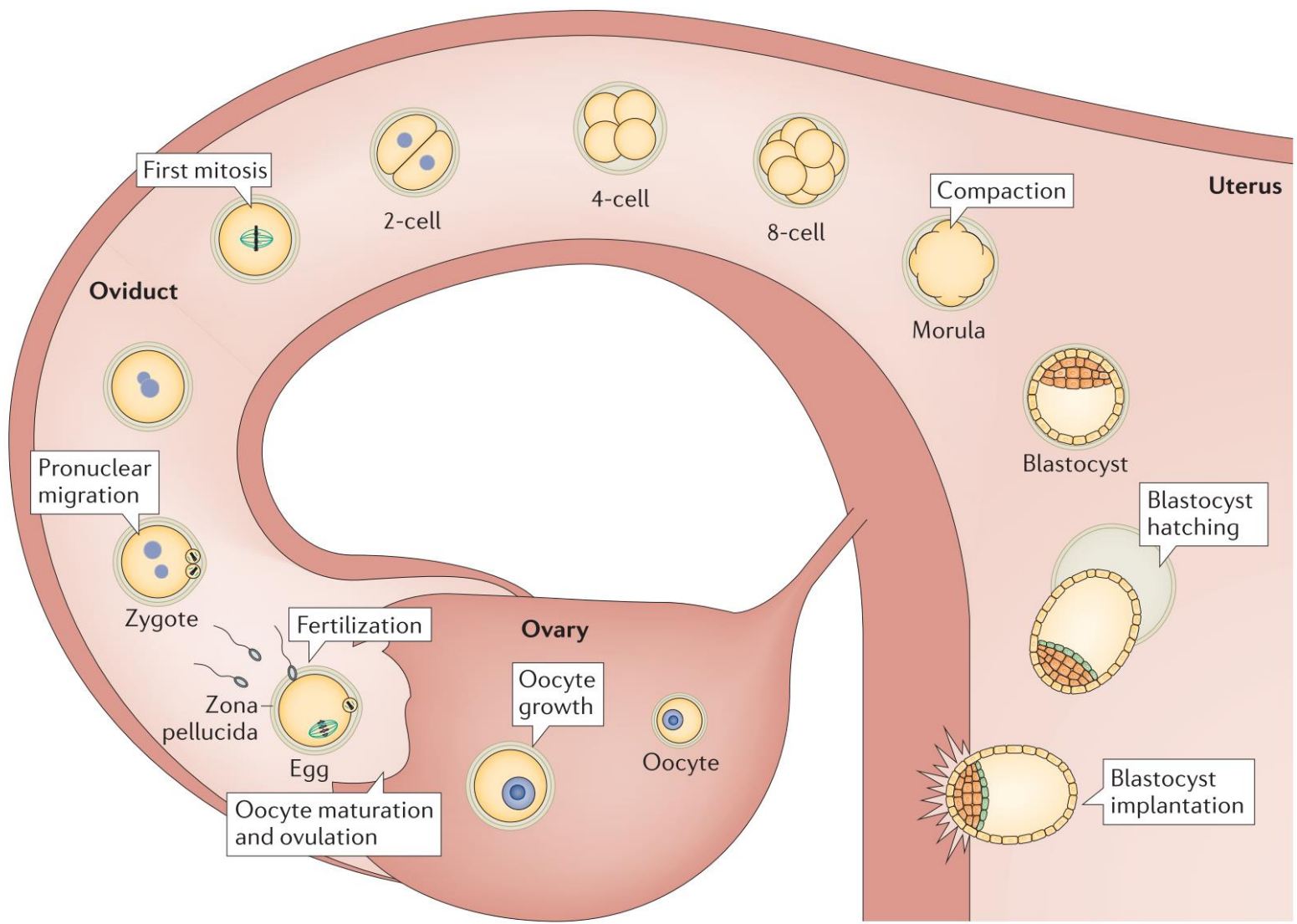

b

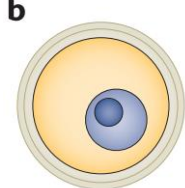

Prophase arrest

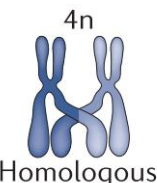

chromosome recombination

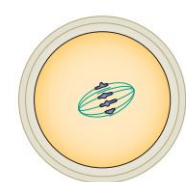

Spindle assembly

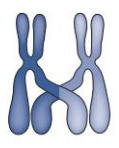

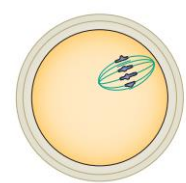

Spindle relocation

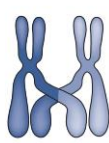

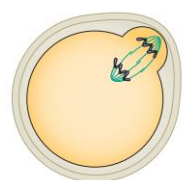

First polar body extrusion

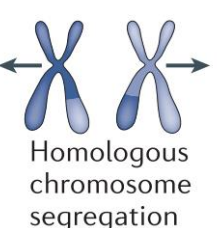

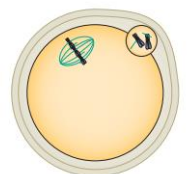

Metaphase II arrest

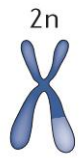

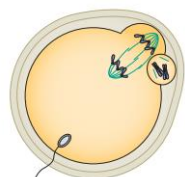

Second polar body extrusion

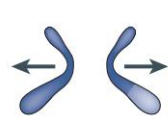

Sister chromatid segregation

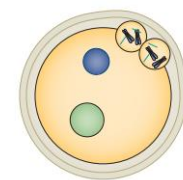

Pronuclei formation

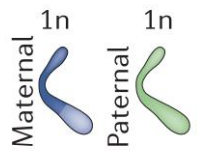

Haploid pronuclei

Figure 1: Overview of mammalian pre-implantation development and oocyte maturation. (A) Progression of preimplantation development from ovulation until blastocyst implantation. (B) Oocyte maturation and female meiosis from prophase arrest to fertilisation and pronuclear formation. From (Clift and Schuh, 2013). 


\subsection{Fertilisation and the oocyte-to-embryo transition}

When an egg is fertilised by a sperm, two highly specialised gametes fuse to form a totipotent zygote, which goes on to give rise to all tissues of the embryo and extraembryonic tissues; an overview of early embryonic development is shown in Figure 1A. For this process to occur successfully, several aspects must be tightly controlled, beginning with monospermic fertilisation, subsequent egg activation, and the many cellular processes that must switch during the cell's transition from an oocyte to early embryo. A summary of these processes, and recent findings that have contributed to this knowledge, will be given in this section.

\section{Fertilisation: when sperm meets egg}

In mammals, ovulation and sexual intercourse resulting in ejaculation of semen into the female genital tract must take place in the same window of time to allow in vivo fertilisation. The ovulated egg is surrounded by a glycoprotein matrix called the zona pellucida, as well as a cloud of somatic cumulus cells in a hyaluronic acid jelly. This cumulus-oocyte complex travels through the oviduct towards the uterus. Following ejaculation of hundreds of millions of sperm, only tens or hundreds reach the egg; during this journey, they undergo capacitation and thus gain the ability to fertilise an egg only after ejaculation (reviewed by Sakkas et al., 2015). The sperm's surface contains hyaluronidases, probably to aid in the sperm's passage through the cumulus cells surrounding the egg to reach the zona pellucida (Kimura et al., 2009). The zona pellucida is an extracellular glycoprotein coat surrounding the mammalian egg; in mouse eggs, it has a thickness of approximately $7 \mu \mathrm{m}$. It consists of several different glycoproteins; in the mouse, three zona pellucida proteins have been identified, ZP1-3, ranging in molecular weight from 60-200 kDa (Bleil and Wassarman, 1980). ZP2 and ZP3 form an array that is cross-linked by ZP1. In humans, a fourth ZP4 has also been found (Lefièvre et al., 2004). These proteins are thought to lead to species specificity in sperm-egg binding, although the level of specificity is variable throughout the animal kingdom (Bedford, 1981). Furthermore, the zona pellucida is critical for preventing polyspermy, as an orchestrated sequence of events modulates its structure. First, the sperm recognises the egg membrane through its surface protein Izumo1 (Inoue et al., 2005), which binds the egg's receptor Juno (Bianchi et al., 2014); this sperm-egg membrane fusion is furthermore dependent on CD9, which may act as a partner of Juno (Chalbi et al., 2014; Jegou et al., 2011; Miyado et al., 2008). During meiotic progression, cortical granules translocate from the oocyte's centre to the plasma membrane in a Rab11a- and myosin-Vb-dependent manner (Cheeseman et al., 2016). These cortical granules and microvilli at the egg surface are excluded from the region directly overlying the MII spindle, to avoid the sperm DNA being captured by the spindle in meiosis II (Deng et al., 2003). A recent report found that the cortical granules modify the zona pellucida following fertilisation; after a sperm binds to the $\mathrm{N}$-terminus of $\mathrm{ZP} 2$, there is a rapid, transient block to sperm 
penetration as zinc sparks are observed, and ovastacin is released from cortical granules (Tokuhiro and Dean, 2018). Ovastacin subsequently cleaves ZP2, rendering the zona pellucida non-permissive for other sperm (Bleil et al., 1981; Burkart et al., 2012; Tokuhiro and Dean, 2018).

Once the sperm has bound the zona pellucida, it releases its acrosome, a specialised vesicle at the tip of the sperm head. The acrosome contains various enzymes that allow the fusion of sperm and egg membranes. With the membranes fused, a cascade of events leads to an increase of free calcium ions and onset of $\mathrm{Ca}^{2+}$ oscillations in the zygote. The very first calcium oscillation initiates at the site of sperm entry and moves through the cell, with subsequent transients becoming more uniform throughout the cell and the period increases from approximately every 11 minutes to 31 minutes (Deguchi et al., 2000). On a mechanistic level, PLC is released from the sperm head into the egg, which in turn induces release of $\mathrm{Ca}^{2+}$ from the endoplasmic reticulum through $\mathrm{IP}_{3}$ (Miyazaki et al., 1992, 1993; Stricker, 1999). This activates CaMKII, which together with PLK1 phosphorylates EMI2, targeting it for degradation (Hansen et al., 2006; Rauh et al., 2005). EMI2 maintains metaphase II arrest of mature eggs through CSF and inhibiting the APC/C (Shoji et al., 2006). Through its degradation, APC/C is activated, while cyclin B and securin are destroyed (Madgwick et al., 2006), resulting in completion of MII and extrusion of the second polar body (reviewed by Bianchi and Wright, 2016; Clift and Schuh, 2013). Interestingly, calcium oscillations continue after polar body extrusion, ceasing only at the onset of interphase, when pronuclei form and pronuclear migration begins (Jones et al., 1995).

Following sperm entry, an actin-rich protrusion, known as the fertilisation cone, forms directly overlaying the paternal DNA and remains there until pronuclei have migrated inwards (Maro et al., 1984). Injection of sperm chromatin into MII oocytes revealed that the formation of this actin enrichment is dependent on the distance between the chromatin and overlying cortex, and requires Ran GTPase (Deng et al., 2007) as well as MAPK and MLCK (Deng et al., 2005). Interestingly, sperm DNA injected under the cortex of MII oocytes is able to induce ectopic polar body extrusion if it is modified to form a spindle (Deng and Li, 2009). The fertilisation cone contracts rhythmically, inducing cytoplasmic motions correlated with the above-mentioned calcium oscillations. Fluorescent markers of actin and myosin-II localise to the shoulders, or base, of the fertilisation cone, and their intensities oscillate with fertilisation cone contractions, indicating that these are actomyosin contractions. Furthermore, treatment of zygotes with MLCK inhibitor ML-7 perturbs these contractions as well as decreasing the protrusion of fertilisation cones. Actomyosin contractions of the fertilisation cone end at the formation of pronuclei (Ajduk et al., 2011). 


\section{Transitioning from oocyte to embryo}

Once the sperm has entered the egg and the polar body has been extruded, the zygote must undergo several cellular changes to allow further embryonic development. The most critical changes are those at the level of the chromatin, and those occurring in the cytoplasm.

On the chromatin level, DNA is first repackaged and undergoes epigenetic reprogramming, followed by zygote genome activation. In the sperm, DNA is tightly condensed by protamines, which are replaced by maternal histones, initially by the histone $\mathrm{H} 3.3$ after fertilisation, then later the canonical histone H3 (Torres-Padilla et al., 2006). Indeed, the histone chaperone Hira is required for male pronucleus formation and development past the zygote stage (Lin et al., 2014). As the pronuclei form, both maternal and paternal chromosomes undergo decondensation, which is dependent on maternal factors. Until recently, specific factors had not been identified, but it is now known that the DNA damage response factor RAD9A plays a role in this decondensation. Mice with an oocyte-specific knock-out of Rad9a are severely subfertile, as DNA replication and pronuclear formation in the zygotes was delayed enough to cause G2/M checkpoint arrest (Huang et al., 2019). Furthermore, as the sperm DNA is highly methylated when it enters the cell (Kobayashi et al., 2012), selective demethylation of paternal DNA must occur (Mayer et al., 2000; Oswald et al., 2000). Although the mechanisms of this fast and active demethylation are not fully understood, it is known that TET3 is a critical protein during this demethylation; Tet3 $\%$ mice are not able to develop normally (Gu et al., 2011). The presence of PGC7 (Stella) on maternal chromatin protects it from this demethylation (Nakamura et al., 2012). Later, during DNA replication before each early embryonic division, passive DNA demethylation occurs on both parental genomes (Rougier et al., 1998). Importantly, the zygotic genome is slowly reactivated after the oocyte has been transcriptionally silent since the end of its growth phase. In the zygote, some transcription occurs from the paternal genome (Aoki et al., 1997), including ribosomal RNA transcription (Lin et al., 2014), although the main activation of transcription occurs in the 2-cell embryo. The genome is activated through maternal factors, notably BRG1, without which transcription of the 2-cell embryo is decreased by $30 \%$, and development fails past the 2 -cell stage (Bultman et al., 2006). As maternal factors deposited in the oocyte prior to its transcriptional quiescence are critical in controlling chromatin remodelling and genome activation, translation and activation of these factors must be well-regulated, but not all mechanisms relating to this tight control are fully understood (reviewed by Clift and Schuh, 2013; Schulz and Harrison, 2019)

In addition to chromatin remodelling, the cytoskeleton must also undergo several changes in its transition from oocyte to embryo. While the majority of the cytoskeleton is contributed by the oocyte, one important feature that the sperm brings to the zygote besides the paternal chromosomes, in many species, is the centriole-containing centrosome. This canonical centrosome is the main microtubule 
organising centre (MTOC) in mitotic cells, and consists of a pair of centrioles surrounded by the pericentriolar material. In many animal species, with exceptions such as starfish, centrioles are lost during oocyte maturation, although proteins of the pericentriolar material often remain. The sperm, on the other hand, retains the centriole in most species (reviewed by Manandhar et al., 2005). As a result, the sperm contributes functioning centrioles in most species including humans; human zygotes already have centriole-containing mitotic spindles at the time of the first embryonic division (Sathananthan et al., 1991). Interestingly, in mouse gametes, centrioles are lost during both oocyte and sperm maturation (Schatten et al., 1985), meaning that neither sperm nor egg contribute centrioles to the zygote (reviewed by Hoyer-Fender, 2012). Thus, the transition from meiotic to mitotic spindle assembly is not immediate in mouse zygotes. In mouse oocytes, the meiotic spindle is nucleated by acentriolar MTOCs (Clift and Schuh, 2015; Schuh and Ellenberg, 2007). Indeed, the first mitotic divisions, including that of the zygote, appear to rely mostly on these aMTOCs. Slowly, the amount of aMTOCs decreases and the shape of the spindle gradually changes, until typical mitotic and centriole-containing spindles are observed in the mouse blastocyst (Courtois et al., 2012).

Before the spindle assembles, pronuclei must first reach the centre; correct pronuclear migration is required to unite maternal and paternal DNA in the centre of the zygote, as well as for correct spindle formation around them, and thus is critical for the first mitotic division (Reichmann et al., 2018; Simerly et al., 1995). Importantly, this process of pronuclear migration is also dependent on the spermderived centrioles in most species, including humans (reviewed by Clift and Schuh, 2013). In mouse zygotes, however, pronuclear migration in the absence of sperm asters must depend on other cytoskeletal mechanisms. While evidence exists to indicate that actin plays an essential role (Chaigne et al., 2016; Maro et al., 1984; will be discussed in detail later), the exact mechanisms of how the pronuclei are able to migrate to the centre in mouse zygotes are not well understood. To provide background for the mechanisms that exist to position nuclei, the next section will first describe how microtubule and actin cytoskeletons contribute to nuclear positioning events in a variety of biological contexts, including pronuclear migration in other species. The current state of knowledge of nuclear positioning in mouse oocytes and zygotes will then be summarised, leading to the open questions that are the basis of this thesis. 


\subsection{The mechanisms of (pro)nuclear migration in different contexts}

The mechanisms that allow nuclei to position themselves and the cytoskeletal structures involved have been studied in a variety of species and biological processes (reviewed by Bone and Starr, 2016; Dupin and Etienne-Manneville, 2011; Lee and Burke, 2018; Lele et al., 2018; Reinsch and Gönczy, 1998). Understanding the mechanisms that have been shown to be important in pronuclear migration of other species, as well as in other biological contexts, can be useful in deciding upon the pathways that may be investigated in pronuclear migration of mouse zygotes, and provide context for any later findings. Thus, the role of cytoskeletal elements in the localisation of nuclei, such as microtubules and centrosomes, as well as the actin cytoskeleton, will be the focus of this next section; an overview is provided in Figure 2. It should be noted that, to date, little evidence of a direct role of intermediate filaments in nuclear positioning exists (reviewed by Dupin and Etienne-Manneville, 2011, and shown in Figure $\mathbf{2 C}$ ), and therefore, these will not be discussed in this section.

A Microtubule-dependent nucleus positioning

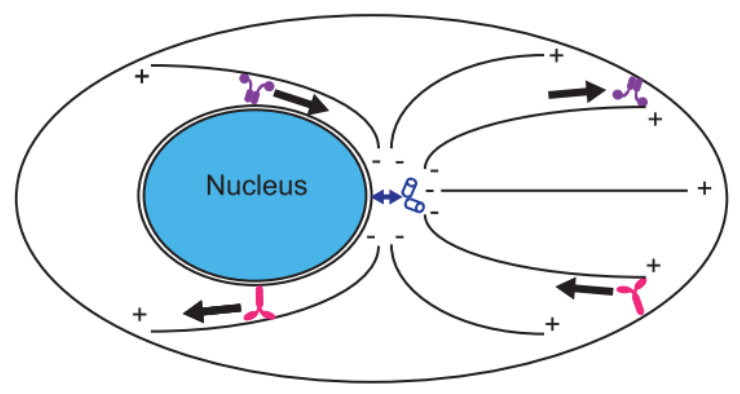

B Actin-dependent nucleus positioning

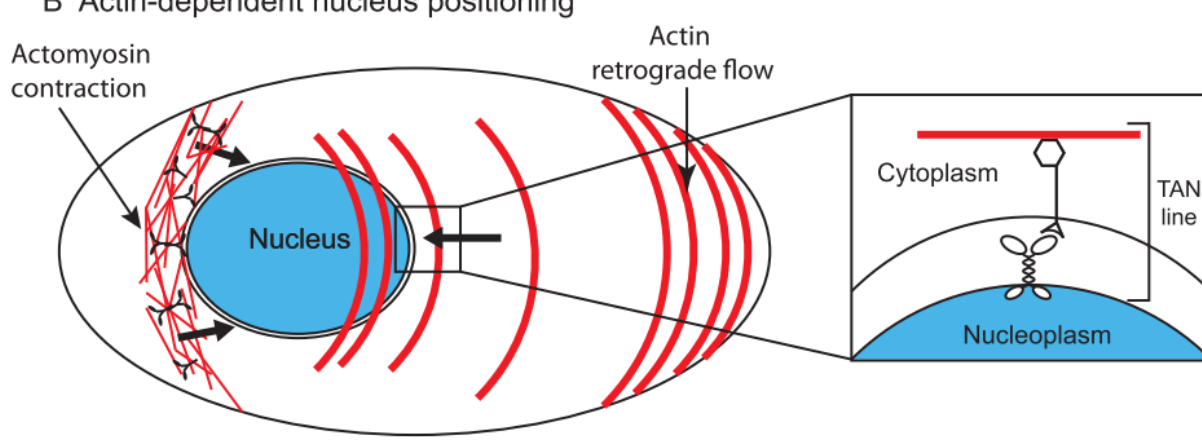

C Intermediate filament-dependent nucleus coupling

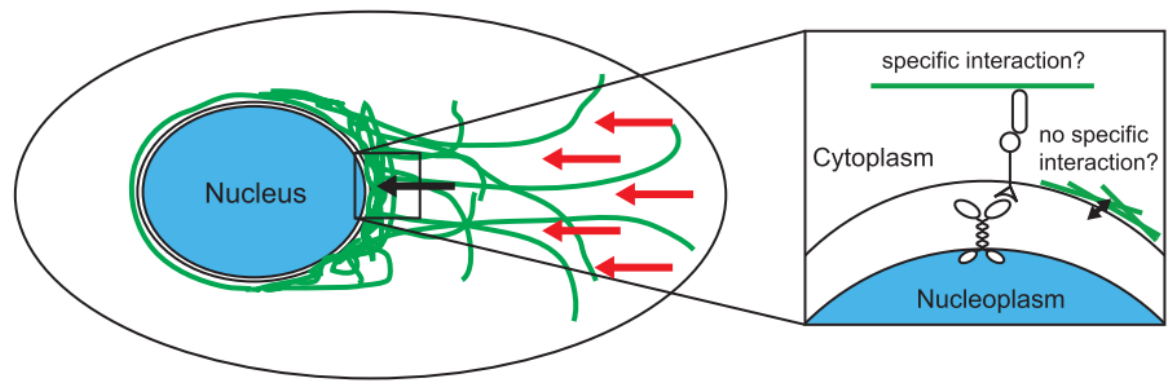

Legends:

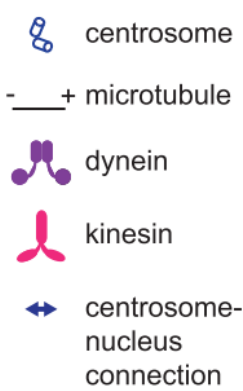

actin

retrograde moving fiber

\myosin II

sun2

9 nesprin-2
- cytoplasmic intermediate filament

\& sun $1 / 2$

† nesprin-3

^ plectin

$\longrightarrow$ actin-dependent 
Figure 2: The role of microtubules, actin and intermediate filaments in nuclear positioning. Forces exerted on the nucleus (blue oval) are depicted by black arrows. Models of (A) microtubule-dependent nucleus positioning, (B) Actin-dependent nucleus positioning and (C) Intermediate filament-dependent nucleus coupling. Legend for key players on right-hand side. Examples of $(\mathbf{A})$ and $(\mathbf{B})$ are described below. From (Dupin and Etienne-Manneville, 2011).

\section{The role of microtubules and centrosomes in nuclear migration}

Microtubules are one of the three main filamentous subsystems of the cytoskeleton. They consist of heterodimers of $\alpha$ - and $\beta$-tubulin, which self-assemble in the presence of ATP. Thirteen of these polymers assemble to form hollow filaments, which have two ends; this polarity is critical to their function. At the minus-end, which is often associated with an MTOC such as the centrosome, $\alpha$-tubulin subunits form the end of the filament; this end has only a low rate of polymerisation. At the plus-end or microtubule end of the filament, $\beta$-tubulin subunits are exposed, and polymerisation can occur at a much faster rate (reviewed by Akhmanova and Steinmetz, 2015; Brouhard and Rice, 2018). Microtubule polymerisation and dynamics are regulated through a vast number of structures and proteins, including Microtubule-Associated-Proteins (MAPs), and, as such, can form complex structures and perform a vast number of functions across cell types (reviewed by Goodson and Jonasson, 2018). Motor proteins such as dynein and kinesins can move along microtubules, thus transmitting pulling or pushing forces to facilitate intracellular transport, cell shape changes, and many other processes (reviewed by Shelley, 2015). Importantly for nuclear migration, these forces can be coupled to the nucleus via LINC complexes, which contain KASH- and SUN-domain proteins that localise to the outer and inner nuclear envelope respectively, and interact in the perinuclear space. In recent years, the composition of LINC complexes has emerged as a critical point of regulation of cellular dynamics (reviewed by Chang et al., 2015a).

In many classical model systems, microtubules and their associated MTOCs, motors and LINC complexes have been found to be key players in nuclear positioning, both in the totipotent zygote and in specialised cells; an overview of the key players and main mechanisms found are shown in Figure 2A. Pronuclei migrate towards each other and to the centre in a microtubule- and dyneindependent manner in the zygotes of many species (reviewed by Clift and Schuh, 2013). The beststudied model organism for such a microtubule-dependent pronuclear migration is the nematode Caenorhabditis elegans (reviewed by Oegema and Hyman, 2006).

Following $C$. elegans fertilisation, the sperm-derived pronucleus sits at the posterior cortex, in close association with the sperm aster, while the female pronucleus usually sits anteriorly in the embryo (Albertson, 1984). The female pronucleus migrates towards the male pronucleus, first slowly and later with a higher velocity (O'Connell et al., 2000). The male pronucleus itself migrates away from the cortex in close association with the sperm aster (Albertson, 1984; O'Connell et al., 2000), possibly by 
a pulling mechanism (Kimura and Onami, 2005). Note that pronuclei are not fully centred, as the first division of $C$. elegans embryos is asymmetric. The migration of both pronuclei is microtubuledependent (Strome and Wood, 1983), although some evidence exists that cortical flows and actomyosin-dependent forces are also important for migration of pronuclei (Goulding et al., 2007; Hird and White, 1993). The microtubule-dependent pathway has been explored in great detail over the last few decades. Pronuclear migration in the $C$. elegans zygote requires LINC complexes to transmit forces and connect the nucleus and cytoplasm. The KASH protein ZYG-12 and SUN-protein SUN-1, together with LIS-1 target dynein to pronuclei (Cockell et al., 2004; Gönczy et al., 1999; Malone et al., 2003). The association between dynein and the nucleus requires the DYRB-1 subunit of the dynein light-chain, as well as Rab7 and RILP (Kimura and Kimura, 2011). It is possible that actin is also involved in this process to allow microtubules to push off the actin cortex, as perturbation of the Arp2/3 complex leads to slowed-down centration of pronuclei (Xiong et al., 2011). Together, these proteins allow correct pronuclear positioning prior to asymmetric division. Importantly, further studies of this nematode have highlighted the importance of the identity of LINC complexes, as dynein is involved in several nuclear migration events of $C$. elegans, not only in pronuclear migration. Interestingly, distinct sets of cargo adaptors have been identified as playing an important role in different developmental events, likely to regulate the exact function of dynein. For example, in the development of hyp7 cells, where nuclear migration has also been well studied, a different KASH protein, UNC-83, interacts with several proteins to regulate the functions of dynein and kinesin-1, and thus facilitate nuclear migration (Fridolfsson et al., 2010). This is a great example of how the intricate details of the identity of LINC complexes can differ between cells in the same organism to allow control of nuclear positioning.

In several other animal species, ranging from classical model systems to large mammals, sperm asters, microtubules and/or dynein have been implicated in zygote pronuclear migration. This includes the sea urchin Lytechinus variegate (Bestor and Schatten, 1981; Chambers, 1939; Schatten and Schatten, 1981; Tanimoto et al., 2016), the sand dollar Clypeaster japonicus (Hamaguchi and Hiramoto, 1986), the fruit fly Drosophila melanogaster (reviewed by Loppin et al., 2015), the zebrafish Danio rerio (Lindeman and Pelegri, 2012; Wühr et al., 2010), the jelly fish Beroe ovata (Rouvière et al., 1994), the frog Xenopus laevis (Stewart-Savage and Grey, 1982; Wühr et al., 2010), the pig Suf scrofa domesticus (Kim et al., 1997), the cow Bos taurus (Navara et al., 1994; Payne et al., 2003), the rhesus monkeys Macaca mulatta (Payne et al., 2003), and Homo sapiens (Simerly et al., 1995). Even though several details of the exact mechanism are still lacking in many of these, such as which LINC complexes or motor proteins are required for the force transmission to position nuclei, the basic mechanisms appear to be highly conserved. Indeed, a general model for pronuclear migration has been proposed, 
in which the sperm aster nucleates long microtubules which push off the cortex, centring the male pronucleus, while pulling the female pronucleus towards the male pronucleus with dynein and/or kinesin (Clift and Schuh, 2013). This model may even apply to non-animal species; for example, in brown algae, the sperm brings an aster and the male pronucleus migrates towards the centre in a microtubule-dependent, actin-independent manner. However, one notable difference was observed in this model organism: the female pronucleus formed at the centre, and therefore did not migrate (Swope and Kropf, 1993).

While pronuclear migration has been established as dependent on microtubules in many species, it is important to note that other nuclear positioning events also rely on microtubules, LINC complexes and/or dyneins and kinesins. For example, experiments investigating eye development of Drosophila melanogaster were some of the first to reveal the functions of LINC complexes in connecting nuclei to the cytoskeleton. The developing eye disc consists of several hundred ommatidia, subunits of photoreceptor and pigment cells, as well as cone cells. During the progression of the morphogenetic furrow, nuclei migrate first basally and then apically within cells (Fischer-Vize and Mosley, 1994; Tomlinson, 1985). This migration has been shown to rely on microtubules and the minus-end-directed motor dynein, which direct the nuclei towards MTOCs at the apical end of the photoreceptor cells (Fan and Ready, 1997). The interaction between the nuclei and microtubule-dynein network relies on KASH- and SUN-proteins, Klarsicht and Klaroid, and nuclear lamin; without this interaction, nuclei lose their association to the apically-positioned MTOCs and remain basal (Kracklauer et al., 2007; MosleyBishop et al., 1999; Patterson et al., 2004). Another model system that has been important in understanding nuclear positioning is the fission yeast Schizosaccharomyces pombe, in which several nuclear migration events depend on microtubules, MTOCs, and minus-end-directed motors. During interphase, the nucleus is centred by microtubules, whose minus-ends are tightly associated with the nucleus through the spindle pole body, the main MTOC of fission yeast; their plus-ends grow dynamically and push against the cortex (Drummond and Cross, 2000; Tran et al., 2001). This interphase centration is dependent on the kinesin-14 Klp2, a minus-end-directed motor. Interestingly, nuclear positioning direction is cell-cycle dependent, and this kinesin is inhibited for correct anaphase and telophase positioning away from the centre (Mana-Capelli et al., 2012). During the reproductive cycle of S. pombe, haploid cells fuse, and their nuclei must congress for karyogamy to occur. This nuclear migration requires microtubules and two minus-end-directed motors; kinesin-14 Klp2, and dynein (Scheffler et al., 2015).

Clearly, many nuclear positioning events rely on microtubules. However, as previously mentioned, mouse oocytes and sperm, and, as a result, mouse zygotes, lack centrioles. Instead of fitting into the sperm-aster-dependent pronuclear migration model described above, actin appears to be the key 
player in mouse nuclear positioning of oocytes and zygotes; the known details will be described in a later section. Although actin has not been implicated in pronuclear migration of any other species studied, several examples of its importance for nuclear positioning in other contexts exist. Some of these cellular processes will be summarised next, as a background to the mechanisms that may allow actin to function in nuclear positioning.

\section{The role of actin in nuclear positioning}

Actin is a major component of the eukaryotic cell cytoskeleton, present in two forms: globular G-actin monomer, and polymeric filamentous F-actin, also known as microfilaments. Microfilaments do not consist of hollow tubes like microtubules, but rather form thinner filaments with a helical twist. However, they are similar to microtubules in that they also show a structural polarity. During nucleation, dimers and trimers of G-actin are first formed spontaneously as bound ATP is hydrolysed; after this initial nucleation, rapid elongation can occur at the plus or barbed end of the filament, while the minus or pointed end shows slower rates of elongation (reviewed by Blanchoin et al., 2014). Several proteins can facilitate this initial polymerisation, and these so-called actin nucleators fall into three categories: the Arp2/3 complex is responsible for branched F-actin networks as part of the "convergent elongation model"; the Formin family of actin nucleators promotes straight, or unbranched, filament growth in what is known as the "tip nucleation model"; and the third group, known as tandem-monomer-binding nucleators, includes the Spire and APC families of proteins, which contain tandem G-actin-binding motifs (reviewed by Firat-Karalar and Welch, 2011). These different families of actin nucleators may also interact with each other, such as Spire and Formin nucleators (Montaville et al., 2014; Pfender et al., 2011), which will be discussed in more detail later. Other actin binding proteins may further affect the dynamics of the actin cytoskeleton, e.g. by capping barbed ends of filaments (Capping protein), severing existing filaments (ADF/Cofilin), or binding G-actin monomers (Profilin) (reviewed by Pollard, 2016). Another subset of actin binding proteins, tropomyosins, can influence myosin recruitment onto actin filaments (reviewed by Pollard, 2016). Myosins are a superfamily of motor proteins that move along actin tracks for a wide range of cellular processes; they were initially found in muscle cells, where actomyosin contractions allow muscle contractions. However, actomyosin contractions have also been found in several other cell types, such as in the fertilisation cone of zygotes as previously mentioned, and they also have a function in intracellular transport along actin tracks (reviewed by Hartman and Spudich, 2012). Lastly, several KASH proteins have been found to contain actin binding domains, and thus, actin may influence nuclear positioning by direct interaction with the nucleus through LINC complexes and retrograde actin flows, or through actomyosin contractions (reviewed by Chang et al., 2015a); an overview of these is shown in Figure 2B. 
Actin has been found to be important in both inhibiting and facilitating migration of nuclei in certain contexts. One well-studied example of its involvement in nuclear migration is during wound healing of cell monolayers. In the intact monolayer, nuclei are centred within their cells. In response to wounding and in the presence of serum, fibroblasts migrate to the wound edge. During this migration, their nuclei are positioned to the rear of the migrating cell. Interestingly, the centrosome remains central as the nucleus migrates, sitting between the nucleus and the leading edge of the cell (reviewed by Chang et al., 2016). Indeed, instead of microtubules positioning the nucleus, transmembrane actinassociated nuclear (TAN) lines have been found to be important for this process (reviewed by Luxton et al., 2011). The first observation in this context was that Cdc42, MRCK, myosin and actin were required for a retrograde actin flow that positioned the nucleus; treatment with blebbistatin or cytochalasin D, or injection of dominant-negative $C d c 42^{\top 17 N}$ or MRCK-CPC/TM proteins, all individually perturbed nuclear migration to the rear of the cell. Taken together, these results implicated myosin-II function in this nuclear migration. On the other hand, dynein and other microtubule-related proteins were not required for nuclear position, but instead for maintenance of the centrosome at the centre (Gomes et al., 2005). Another report found that nesprin-2, a KASH protein, as well as SUN2, were required for rearward migration of nuclei in migrating fibroblasts; overexpression of the KASH domain of nesprin-2, nesprin-2G siRNA inhibition, and SUN2 depletion all inhibited the migration, while reexpression of nesprin-2G was able to rescue the siRNA phenotype. Furthermore, fluorescently tagged nesprin-2G constructs and endogenous SUN2 showed linear arrays co-localizing with actin cables, which were dependent on actin and myosin-II; these arrays were named TAN lines (Luxton et al., 2010). More recent reports have focused on the direct links between the nucleus, TAN lines, and the actin cables by investigating interactions of the LINC complexes at the nucleus and actin cables. Anchorage of nesprin-2G-SUN2 TAN lines was found to require several proteins of the nuclear envelope: Lamin A (Folker et al., 2011), Samp1 (Borrego-Pinto et al., 2012), and Emerin (Chang et al., 2013). To connect the LINC complex TAN lines to the actin cables, two different actin-binding or nucleating proteins have been investigated. First, the diaphanous formin FHOD1 was found to be required for nuclear migration in wound healing, although its silencing still allowed rearward actin flows, indicating that multiple connectors were required for force transmission (Kutscheidt et al., 2014). Indeed, the actin-binding protein Fascin has also been found to facilitate the connection between nesprin-2G-SUN2 TAN lines and the actin cytoskeleton (Jayo et al., 2016). Finally, this nuclear positioning has been found to be required for positioning of this centrosome and thus orientation of cell migration (Chang et al., 2015b). Fibroblast wound healing has thus been a useful model to explore how actin can facilitate nuclear migration through LINC complexes. 
Actin has also been found to facilitate nuclear migration through actomyosin contractions in several other migrating cell types, in particular various neuronal precursor cells. However, until now, TAN lines as described above have not been found in these systems, and in general, these processes have not yet been described in as much detail. During migration of cortical interneurons in rodent brains, the nucleus migrates to the leading process of the cell, in contrast to the rearward movement described in wound healing. F-actin and myosin II accumulations were described behind the nucleus (Bellion et al., 2005; Schaar and McConnell, 2005). More recently, actin was shown to cycle dynamically in close proximity to the nucleus, at times forming a cup-like structure. Myosin was found to show a similar localisation, and inhibition of actomyosin contractions with blebbistatin inhibited actin accumulation and nuclear migration. The same study found that depolymerisation of microtubules with nocodazole did not impact neuronal migration, but freezing of the microtubule network with Taxol did, indicating that a dynamic microtubule network is required for actomyosin-induced forces to translocate the nucleus to the leading edge of the cell (Martini and Valdeolmillos, 2010). Furthermore, actin-related proteins DISC1, Girdin, Akt, and mDia1 and mDia3 have all been found to be involved in this actindependent nuclear movement (Shinohara et al., 2012; Steinecke et al., 2014). Similar to these migrating neuronal cells, blebbistatin also inhibited apical-to-basal interkinetic neuronal migration in progenitor cells of the ventricular zone, as well during delamination of cells in the subventricular zone of the developing mouse brain (Schenk et al., 2009), implicating actomyosin contractions in these nuclear migration events. Finally, neural precursor cells of the neocortex must migrate along radial glial fibre cells, and their centrosomes and nuclei move continuously during this migration. In this process, dynein and LIS1 were found to be crucial for both centrosome and nuclear migration, while myosin-II was only required to push the nucleus, and not the centrosome, forward (Tsai et al., 2007). Evidently, actin and myosin are implicated in several different nuclear migration processes, although not all have been described in great detail.

In other processes, actin-dependent nuclear positioning has been described as independent of both actomyosin contractions and LINC complexes. In the syncytial Drosophila embryo, an oocyte is surrounded by 15 interconnected nurse cells, which synthesise RNA and protein for the developing oocyte. These products are "dumped" into the cytoplasm of the oocyte towards the end of its maturation. However, for this dumping to be possible, nuclei of the nurse cells must be positioned away from the interconnecting ring canals in order to avoid blocking the connection to the oocyte (Spradling, 1993). Indeed, research found that actin cables were present and required to keep the nuclei from clogging the ring canals (Cooley et al., 1992; Robinson and Cooley, 2002). The LINC complex proteins found to be important in Drosophila eye development mentioned previously, Klarsicht and Klaroid, as well as another KASH-protein, Msp-300, were not found to be essential for 
this process (Technau and Roth, 2008). It should be noted that it is still possible that undiscovered and thus untested LINC complex proteins, or indeed myosin, could still potentially be involved in this process; however, no such links have been found to date. Until recently, the conclusion from these results was that actin cables formed ladder-like structures required for maintaining nuclei in their position, as opposed to actively positioning them (Dupin and Etienne-Manneville, 2011; Guild et al., 1997). However, advances in live cell imaging allowed a more recent study to challenge this conclusion, instead proposing that actin cables act as inward-facing filopodia-like structures, and actively move the nuclei to their position away from the ring canals. These actin cables were found to elongate into the cytoplasm, towards the nucleus of the cell from the sides closest to the ring canals, thus pushing the nucleus away, with the perinuclear plus ends in close association with the nucleus. Furthermore, this study described a perinuclear actin network in nurse cells that was present before actin cable elongation, although the exact function and interaction with actin cables could not be tested (Huelsmann et al., 2013). This example highlights that developments in live cell microscopy can allow new insights into the dynamics and functions of actin in nuclear positioning, although functional tests of specific actin structures are still limited.

\section{Nuclear migration without a centrosome: mouse oocytes and zygotes}

Although some examples of actin-related nuclear positioning have been identified, zygotic pronuclear migration in the majority of studied species relies on microtubules and centrosomes. As stated previously, rodent oocytes and zygotes lack canonical centrosomes, and therefore must utilise other cytoskeletal networks to control nuclear positioning.

Actin is involved in several processes in the maturing mouse oocyte, including vesicle trafficking, centring the GV nucleus, spindle migration to the cortex, polar body extrusion, as well as accuracy of chromosome segregation (reviewed by Uraji et al., 2018). In particular, the function of actin in controlling the position of DNA first to the centre in nuclear positioning, and later to the cortex in spindle migration, is of interest in the context of nuclear migration.

During the growth of GV oocytes, the nucleus moves from an off-centred location to the centre of the immature oocyte prior to nuclear envelope breakdown (Brunet and Maro, 2007). A recent study investigated the mechanisms at play in more detail (Almonacid et al., 2015). Oocytes from mice with a knock-out of the actin nucleator Formin-2 show an off-centred oocyte, a phenotype that is rescued with overexpression of Formin-2. However, oocytes with an already-centred nucleus and treated with actin depolymerising drugs showed no off-centring of the nucleus, indicating that the actin cytoskeleton nucleated by Formin-2 is only required for centration, and not maintenance of this position. Live-cell imaging with GFP-tagged Utrophin and subsequent tracking of actin nodes/vesicles 
revealed an activity gradient; vesicles moved with higher local velocity at the periphery of the cell than in the centre. Inhibition of actin vesicle movement with a dominant-negative form of their associated motor protein myosin-Vb blocked GV centration. Perturbation of microtubules with nocodazole, and myosin-II with ML-7, did not affect nucleus centration in mouse oocytes. Thus, it was proposed that this vesicle activity gradient creates a pressure gradient and thus a propulsion force on the nucleus, centring it in an actin-dependent but microtubule- and myosin-independent manner (Almonacid et al., 2015, 2018).

While Formin-2 and actin vesicles appear to be critical for centring the nucleus in GV oocytes, they act to facilitate spindle migration to the cortex during MI. As the GV nucleus is centred at nuclear envelope breakdown, the spindle forming around the chromosomes must migrate to the cortex in order for asymmetric division to occur. Similar to nuclear migration in many contexts, spindle migration in mitotic cells is largely dependent on microtubules (reviewed by McNally, 2013). However, spindle relocation to the cortex in mouse oocytes has been found to require a Formin-2-, Spire2- and Rab11adependent dynamic actin cytoskeleton (Dumont et al., 2007a; Holubcová et al., 2013; Pfender et al., 2011; Schuh, 2013; Schuh and Ellenberg, 2008), as well as a pulling force along the actin filaments exerted by myosin-II (Holubcová et al., 2013; Schuh and Ellenberg, 2008). More specifically, Rab11a vesicles sit at the nodes of the actin network and travel along the filaments towards the periphery in a myosin-Vb dependent manner (Schuh, 2013), while also acting to sequester actin nucleators Formin2 and Spire2 (Holubcová et al., 2013). Formin-2 and Spire2, in turn, work together to nucleate the actin cytoskeleton. Perturbation of any of these elements - Rab11a with a dominant-negative variant or brefeldin A, or Formin-2 or Spire1/2 with siRNA - perturbed spindle relocation to the cortex and altered the dynamics of the cytoplasmic actin network (Holubcová et al., 2013; Pfender et al., 2011). Thus, these three actin-related proteins work together to establish a dynamic actin mesh, which is used by myosin-II to pull the spindle to the cortex (Schuh and Ellenberg, 2008). Spindle migration has also been found to be dependent on cortical thickening nucleated by Arp2/3, exclusion of myosin-II from the cortex, and subsequent cortical softening (Chaigne et al., 2013; Larson et al., 2010), as well as possible Formin-2 accumulations and Arp2/3-dependent cytoplasmic streaming (Li et al., 2008; Sun et al., 2011; Yi et al., 2013). Thus, several different actin-related mechanisms are at work during the migration of the meiotic spindle to the cortex.

In addition to what is known about actin-dependent DNA migration events in oocytes, some reports have investigated pronuclear migration in mouse zygotes. Here, microtubules may be involved with correct pronuclear formation (Schatten et al., 1985, 1989), but the actin cytoskeleton appears to be the main driver of pronuclear migration. Depolymerising microtubules with nocodazole had only a mild effect on centration of pronuclei (Reichmann et al., 2018), but treatment with the F-actin- 
disrupting drugs cytochalasin $D$ and latrunculin-A completely inhibited pronuclear migration and spindle centration (Chaigne et al., 2016; Chew et al., 2012; Maro et al., 1984; Schatten et al., 1986). Similarly, perturbation of the actin-regulating subcortical maternal complex (SCMC) caused asymmetric spindle positioning and division in mouse zygotes, although pronuclear migration was not directly investigated in this study (Yu et al., 2014). The most recent study into spindle centration in mouse zygotes (Chaigne et al., 2016) contained several interesting observations, in addition to confirming that the actin cytoskeleton, in general, was essential for pronuclear migration. First of all, inhibition of myosin- $\mathrm{Vb}$, the motor protein involved with the transportation of actin (Rab11a) vesicles, also perturbed pronuclear migration, although it also caused some morphogenic defects of the cells. Next, live cell imaging of EGFP-Utrophin in mouse zygotes indicated that actin was enriched between the cell surface and pronuclei, although this was not confirmed in fixed cells. From these experiments, it has been proposed that a gradient of actin vesicles, as opposed to an activity gradient as in the GV oocyte, is required for pronuclear migration; more vesicles are present between the pronucleus and the cortex than between the pronucleus and the centre of the zygote, thus creating a pushing force on the pronuclei (Almonacid et al., 2018; Chaigne et al., 2016). However, localisation of actin vesicles was not confirmed in either live or fixed cells beyond EGFP-Utrophin imaging, and was not tracked over time. Thus, the precise localisation and function of actin vesicles and nucleators, and their mechanistic role in pronuclear migration, had not been fully investigated. In general, an investigation of mouse pronuclear migration with high temporospatial resolution was also lacking, as previous experiments focused on fixed cells or imaging of live cells only at the beginning and/or end of pronuclear migration.

\section{A novel set of tools to investigate pronuclear migration in mouse zygotes}

With this in mind, Kathleen Scheffler began her postdoctoral project in the Department of Meiosis in 2015. Her goal was to characterise pronuclear migration in mouse zygotes in high detail, and I joined the project in December 2017 to help achieve this goal. At the time of writing this thesis, our collected results have been prepared into a manuscript, but are not yet published. Therefore, this next introduction section will summarise the unpublished results Kathleen Scheffler had at the time I joined the project to give sufficient background of the methods used and cytoskeletal players of interest. Note that some figures from the prepared manuscript will be shown here, while others will be shown in the Appendix as indicated.

Kathleen Scheffler first established a method to track pronuclear migration from shortly after fertilisation, through pronuclear formation and migration, until after nuclear envelope breakdown. As this protocol was also used for several results shown in this thesis, it is described in detail in the Methods section. In short, MII oocytes were collected from the oviducts of superovulated mice, 
injected with mRNA to express H2B-mCherry (DNA) and MyrGFP (membrane). During the expression time of 3 hours, cauda were collected from male mice and the sperm was capacitated. IVF was set up following expression and capacitation, and zygotes were washed out from sperm after two hours of insemination. Zygotes were then imaged every 15 minutes, with a z-stack of $28 \times 3 \mu \mathrm{m}$, overnight (18 hours). Resulting images were reconstructed in 3D using IMARIS software, allowing tracking of pronuclei relative to the centre of zygote, as well as calculations of velocities. The results of these initial experiments are shown in Figure 3.

A

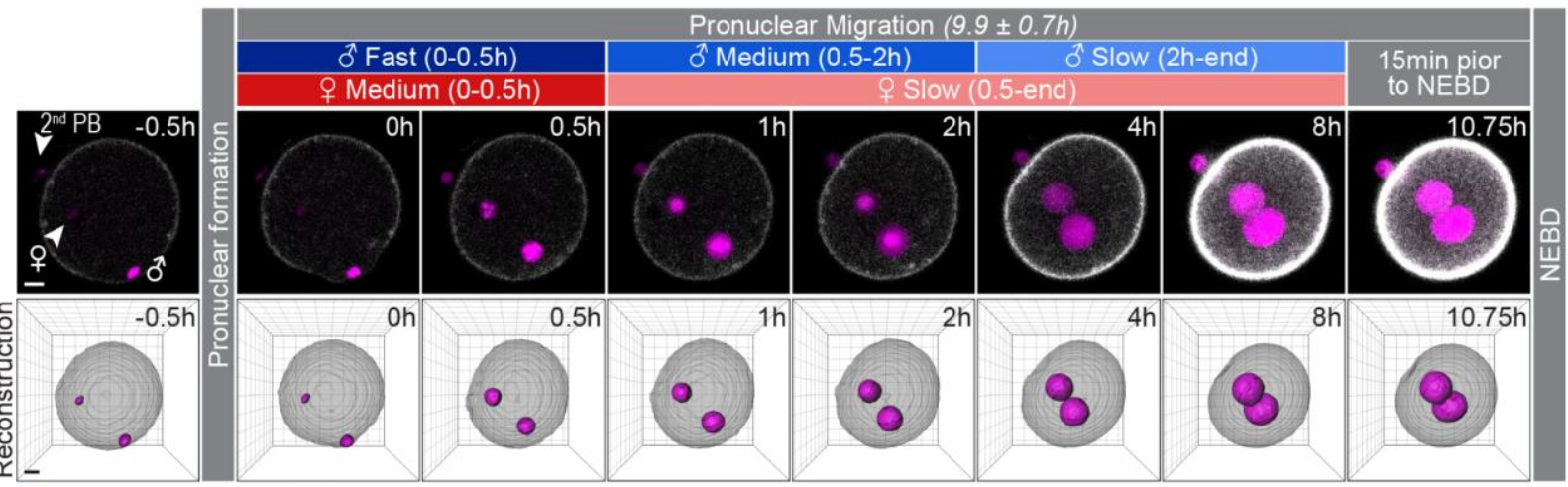
H2B-mCherry (DNA) $\square$ MyrGFP (membrane)

$\mathrm{B}$

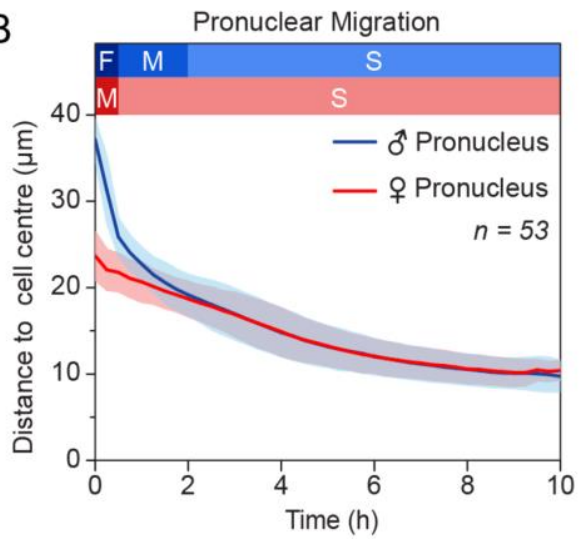

C

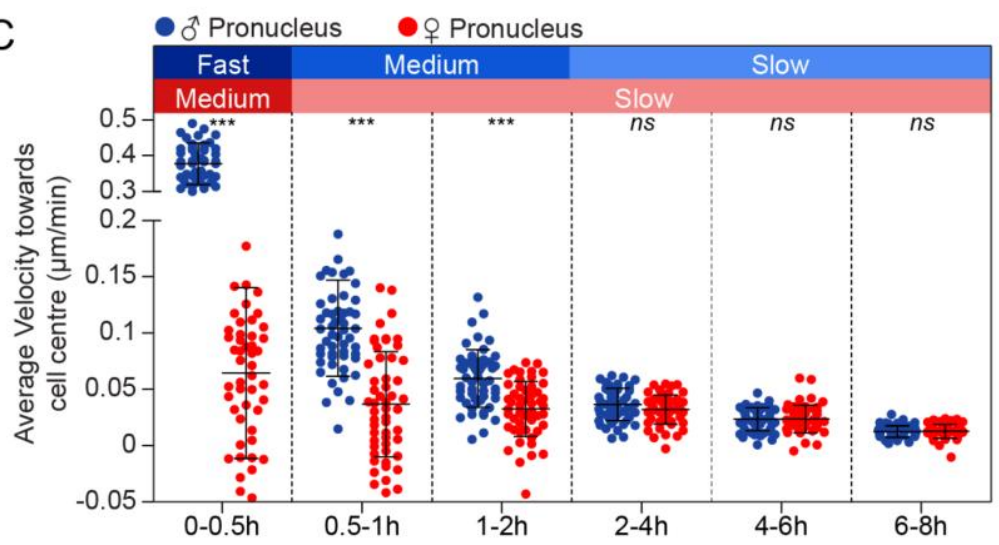

Figure 3: 3D-tracking pronuclear migration in mouse zygotes over time. (A) Three-dimensional time-lapse images (upper panel) and iso-surface reconstruction (lower panel) of pronuclei (H2B-mCherry, magenta, z-projection of 28 section, every $3 \mu \mathrm{m}$ ) and the cell surface (MyrGFP, grey, single z-section) in live mouse zygotes. Female ( $)$ ) and male ( $\left.0^{\prime \prime}\right)$ genomes are shown before pronuclear formation (time-point $O h)$. Movement speeds are classified as fast $(F,>0.1 \mu \mathrm{m} / \mathrm{min})$, medium $(M, 0.05-$ $0.1 \mu \mathrm{m} / \mathrm{min}$ ) and slow $(S,<0.05 \mu \mathrm{m})$. Scale bar, $10 \mu \mathrm{m}$. (B) The mean distance of male ( $O$, blue) and female $(q$, red) pronuclei to zygote centre during pronuclear migration was calculated from data sets as shown in (A). Movement speeds are classified as in (A). Total number of analysed zygotes specified in italics was pooled from five independent experiments. Standard deviation shown as shaded areas. (C) Statistical plots of average velocities of male ( $O$, blue) and female ( $q$, red) pronuclei during pronuclear migration calculated from data sets shown in (B). Movement speeds are classified as in (A). Statistical plots show mean (horizontal black lines), and standard deviation (whiskers). Two-tailed student's t-test was used to test for significance. Adapted from figure prepared for the unpublished manuscript; experiments and analysis done by Kathleen Scheffler. 
Representative confocal images of a zygote over time are shown in Figure 3A. The results of these were quantified, and distances of pronuclei relative to the cell centre were plotted over time in Figure 3B. The first derivative of these results, i.e. the average velocity of pronuclei in different time frames, was calculated and plotted in Figure $\mathbf{3 C}$. Note that the same types of quantification were done in several results figures shown later, with the figure layout and colour scheme adapted for my thesis. One clear observation made from these experiments was that the male pronucleus formed much closer to the cortex (or further from the zygote's centre) than the female pronucleus. This made sense, as the sperm entry site was at the cortex, while the female pronucleus formed wherever polar body extrusion had left the remaining maternal DNA, usually closer to the centre of the cell. Strikingly, this imaging with high temporal resolution revealed that the male pronuclei migrated faster in the first two hours after pronuclear formation than female pronuclei, as shown in the confocal images of Figure $3 \mathrm{~A}$ from $0-2 \mathrm{~h}$, and quantified in Figure $3 \mathrm{~B}$ and $\mathrm{C}$. After the initial two hours, the pronuclei move slowly towards the centre at similar velocities.

Kathleen Scheffler then confirmed the known effect of cytochalasin D as described above using this set-up; the results are shown in Figure $\mathbf{2 7}$ in the appendix. As mentioned, cytochalasin D was known to perturb pronuclear migration, and this was confirmed; two female pronuclei formed as polar body extrusion failed, and these, along with the single male pronucleus, all showed only very limited, if any, migration towards the centre throughout the time of imaging. Kathleen Scheffler also investigated the contribution of microtubules to pronuclear migration by treating zygotes with $1 \mu \mathrm{M}$ nocodazole, as shown in Figure $\mathbf{2 8}$ in the appendix. One interesting observation that is relevant to this thesis is that female pronuclei would often form closer to the cortex in zygotes treated with nocodazole than in the DMSO-treated controls. This could possibly be due to perturbation of the spindle remnant, which may function to keep the female DNA in place after polar body extrusion. Interestingly, as a result, the female pronuclei formed at the cortex and migrated with a high inward velocity in the first few hours, similar to male pronuclei in control cells. Thus, we had a method to induce formation of female pronuclei at the cortex, which will be relevant later. Note that in these experiments late migration of pronuclei was not perturbed significantly, but Kathleen Scheffler later found that this lower concentration of nocodazole did not perturb all microtubules, while a concentration of $10 \mu \mathrm{M}$ nocodazole did; as a result of treatment at the higher concentration, pronuclei moved slower in the later phases of pronuclear migration (data not shown). This higher concentration was therefore also used in experiments shown in the results section of this thesis.

In order to determine which specific actin-related mechanisms may be important for pronuclear migration, Kathleen Scheffler also perturbed formation of recycling vesicles with brefeldin $A$, as previously carried out in oocytes (Holubcová et al., 2013). These results are shown in Figure 4. As can 
be seen in both the confocal images and quantifications, the main effect of brefeldin A treatment was that the male pronuclei, still forming at the cortex, showed a slower initial migration into the centre than male pronuclei in methanol-treated cells. Another observation from these experiments was that brefeldin A perturbed structures inside the cell, since MyrGFP-positive clusters were seen in the cytoplasm (indicated by yellow arrow). Possibly as a result of this, male and female pronuclei were not able to reach as close to the centre in brefeldin A treated cells as in methanol-treated cells. Since brefeldin A causes disassembly of the Golgi network, it is likely that certain structures were aggregating in the centre of the cell, blocking pronuclear migration. Importantly however, these results implied that a brefeldin A-related target functions specifically during the early, fast migration of pronuclei away from the cortex, as only migration of male pronuclei was affected significantly.
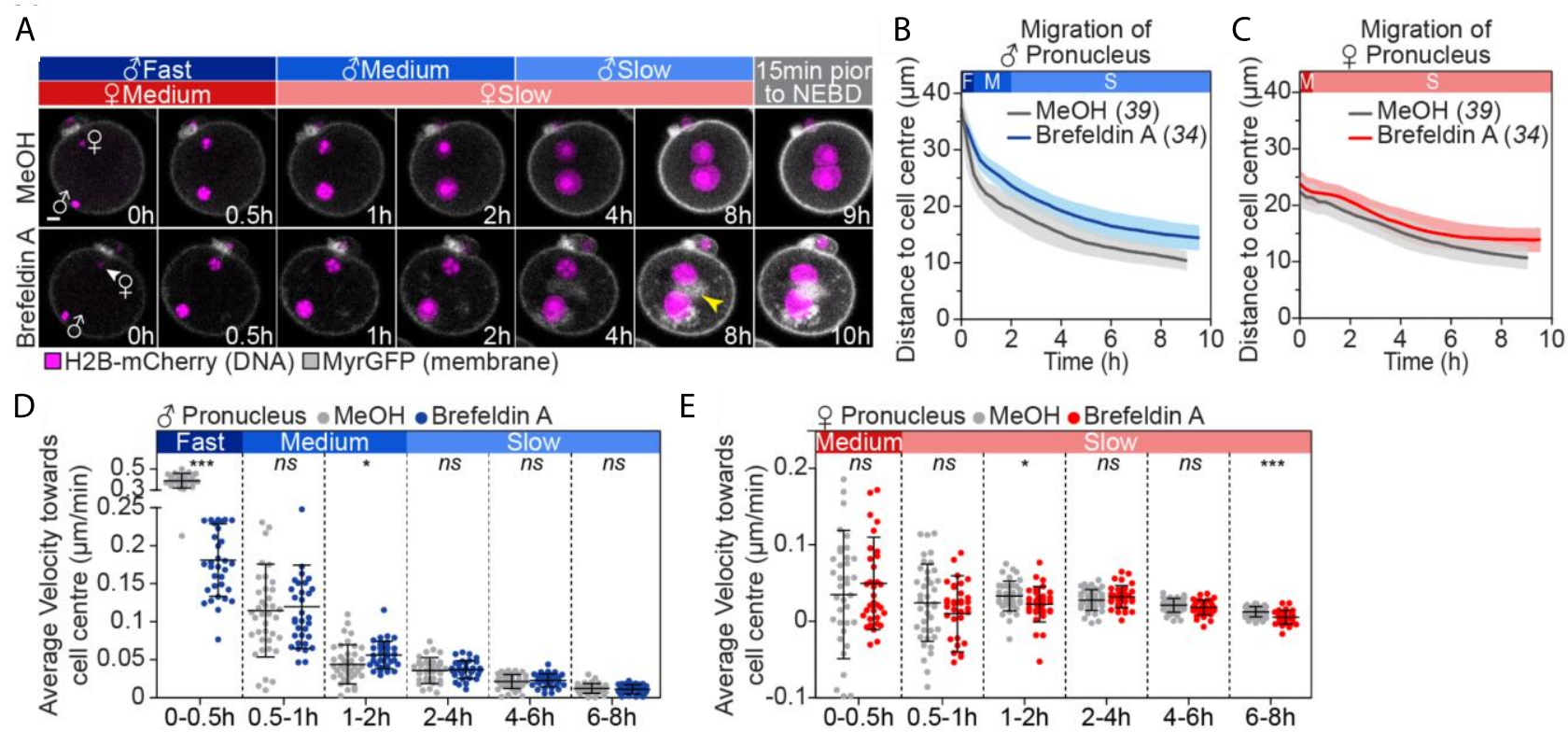

Figure 4: Brefeldin A treatment perturbs the early, fast migration of the male pronucleus. (A) Three-dimensional time-lapse images of pronuclei (H2B-mCherry, magenta) and the cell surface (MyrGFP, white) in methanol (MeOH)- or brefeldin Atreated $\left(4 \mu \mathrm{g} / \mathrm{mL}\right.$ ) zygotes. Female ( $f$ ) and male ( $\left.\sigma^{2}\right)$ genomes are shown at pronuclear formation (time-point Oh). Movement speeds are classified as in Figure 3. The yellow arrowhead marks the increasing accumulation of membranous material in the cell centre, which acts as a barrier for pronuclei to reach cell centre. Scale bar, $10 \mu \mathrm{m}$. (B, C) The mean distance (thick line) of male $(O,(B))$ and female $(f,(C))$ pronuclei to zygote centre during pronuclear migration $\mathrm{MeOH}$-or brefeldin A-treated zygotes was calculated from data sets as shown in (A). Movement speeds are classified as in Figure 3. The total number of analysed zygotes specified in italics was pooled from three independent experiments. Standard deviation shown as shaded areas. $(D$, E) Statistical plots of average velocities of male $(O, D)$ and female $(q, E)$ pronuclei during pronuclear migration in $\mathrm{MeOH}$ - or brefeldin A-treated zygotes calculated from (B) and (C). Movement speeds and statistical plots are classified as in Figure 3. Two-tailed student's $t$-test was used to test for significance. Adapted from figure prepared for the unpublished manuscript; experiments and analysis done by Kathleen Scheffler. 
Taken together, these initial results indicated that two separate mechanisms may be at play to allow correct pronuclear migration into the centre in acentrosomal mouse zygotes - one actin-dependent mechanism active during the early, fast migration of pronuclei, and possibly actin-related vesicles; the second mechanism more dependent on microtubules, and possibly vesicle-independent actin structures. Thus, we then split the project, although overlap remained as we progressed. Kathleen Scheffler focused mainly on the later stages of pronuclear migration, including the function of microtubules for this late, slow migration. My focus became the early, fast migration, in particular of the male pronucleus. As brefeldin A is known to perturb vesicle formation, and Rab11a is a known regulator of actin-related vesicles in oocytes, my focus became to investigate the role of actin, specifically actin-related Rab11a vesicles and actin nucleators during this migration. The next section of this introduction will provide a background for the specific actin-related proteins and functions investigated in this thesis, and the tools available to perturb them. 


\subsection{Rab11a, Formin and Spire}

\section{Rab11a protein and vesicles}

Many types of proteins are involved in the formation of vesicles, such as cytosolic coat molecules for budding, SNARE complexes that control membrane fusion, the microtubule and actin networks on which they travel, and the motor proteins that facilitate their transport along these tracks. Another class of proteins has been identified that is responsible for vesicle identity, and their subsequent interactions, dynamics and functions: Rab GTPases. They are members of the Ras superfamily of small G proteins, and around 70 different Rab GTPases have been identified in humans. They are found either in their GTP-bound, usually active form, or their GDP-bound, usually inactive form. Rab protein function is regulated by several other classes of proteins, making their functions very versatile and dynamic. GEFs and GAPs regulate exchange of GDP to GTP and back, while GDls prevent GDP dissociation from the Rab GTPase, and are crucial for several functions such as regulating chaperoning, mediating delivery, and recycling. GDIs, in turn, are displaced from Rab proteins by GDFs. All of these regulatory steps in combination with the variety of Rab proteins involved in a vast number of cellular events lead to a high level of complexity in vesicle functions (reviewed by Stenmark, 2009).

Rab11 GTPases are a small sub-family of Rab proteins involved in targeting vesicles from the recycling endosome to the plasma membrane. Several Rab11 proteins have been described, notably Rab11a, Rab11b, and Rab25. They share high sequence homology, and are sometimes found to have very similar functions in cells, such as Rab11a and Rab25 in canine kidney cell apical recycling and transcytosis (Wang et al., 2000b). However, in other cell types, different Rab11 proteins show distinct localisation and function, such as in rabbit epithelial cells (Lapierre et al., 2003). In general, Rab11a is the best-studied of the Rab11 family proteins. Rab11a is a GTPase with a length of 216 amino acids and an atomic mass of $24 \mathrm{kDa}$. Reports reveal that Rab11a is responsible for targeting vesicles containing many different cargos to the plasma membrane, whether physiological or in disease state. For example, while Rab11a-knockout mice die as embryos, those with conditional knock-outs for the intestine live one week past birth, with defects in apical protein transport. On the other hand, a conditional Rab11a-knockout in the brain causes no obvious defects (Sobajima et al., 2015). In disease, Rab11a is a host factor for infections of the influenza virus, aiding in transport of the viral ribonucleoprotein complex to the plasma membrane (Amorim et al., 2011; Eisfeld et al., 2011), as well as being required for correct parasitic cytokinesis (Agop-Nersesian et al., 2009). As mentioned previously, Rab proteins can aid in targeting vesicles to the correct cytoskeletal tracks or motor proteins. Experimental evidence has long suggested that myosin- $\mathrm{Vb}$, which travels on actin tracks, is involved with Rab11dependent recycling endosome transport (Roland et al., 2007; Schuh, 2013; Wang et al., 2008b). Recently, an in vitro study revealed that Rab11a, through the adaptor Rab11FIP2, binds to the globular 
tail domain of myosin- $\mathrm{Vb}$ and thus modulates its motor function (Ji et al., 2019). It therefore makes sense that Rab11 has been implicated in various actin-related processes. Indeed, the studies relating to spindle migration in the mouse oocyte mentioned in the previous section revealed that Rab11a not only travels along actin tracks in a myosin-Vb-dependent manner, but by sequestering actin nucleators at the vesicle surface, actively modulates the actin cytoskeleton (Holubcová et al., 2013; Schuh, 2013). Interestingly, a recent in vitro study of the human protein variants found that Rab11 interacts with Spire2 not directly but through myosin-V, forming a Spir-2:MyoVa:Rab11 complex. The Spir-2 binding pocket of myosin-Va appears to be conserved in myosin-Vb, and Spir-2 may drive Rab11 activity (Pylypenko et al., 2016). Whether such a Spir2:MyoVb:Rab11 complex forms in vivo and in mouse remains to be clarified. A function of Rab11 as an actin remodelling protein has also been shown in other systems, such as during Drosophila furrow formation (Riggs et al., 2003), Drosophila wing development (Bhuin and Roy, 2011), and in human T-cell activation through Rac-1 (Bouchet et al., 2016).

Two main types of perturbations have been used for published studies investigating Rab11a: the pharmacological method of brefeldin A treatment, which was also used by Kathleen Scheffler as mentioned above, and overexpression of mutant Rab11a variants.

Brefeldin $A$ is a fungal toxin known to inhibit protein trafficking and the secretory pathway at the level of the Golgi and endoplasmic reticulum (reviewed by Pelham B, 1991), although the exact mechanisms unravelled later (reviewed by Nebenfuhr et al., 2002). In the Golgi, Sec7-type GEFs bind to and activate ARF GTPases by catalysing the exchange of GDP and GTP (Jackson and Casanova, 2000; Spang et al., 2001). Arf1p is a protein involved in coat protein recruitment for vesicle formation. Brefeldin A binds to the transitory Sec7d-Arf1-GDP complex, stabilising this confirmation and blocking activation of Arf1p (Robineau et al., 2000). Thus, coat protein recruitment fails, and the Golgi membrane collapses into the endoplasmic reticulum membrane, releasing its enzymes, while the trans-Golgi network merges with endocytic pathway components (Sciaky et al., 1997). As a result, Golgi trafficking fails, and several cellular processes are perturbed. Importantly, brefeldin A has previously been used to perturb spindle migration in mouse oocytes (Wang et al., 2008a), and more specifically to inhibit mCherry-Rab11a-positive vesicle formation, thus affecting the dynamic actin cytoskeleton (Holubcová et al., 2013). Thus, brefeldin A is one tool used to perturb Rab11a function in animal cells, as well as other cellular processes.

In order to study Rab11a function more specifically, several different Rab11a mutant variants have been described, notably Rab11 $a^{525 \mathrm{~N}}$ and Rab11a ${ }^{\mathrm{Q} 70 \mathrm{~L}}$. Rab11 $\mathrm{a}^{\mathrm{S25N}}$ is a dominant-negative variant; as a result of an exchange of serine 25 to asparagine, this Rab11a variant prefers the GDP-bound, inactive 
state. On the other hand, Rab11a $a^{Q 70 L}$ is a constitutively active form of Rab11a, preferring the GTPbound state. Thus, these two variants both perturb the cycling of Rab11a between its active and inactive form, and thereby perturb transferrin recycling to the plasma membrane in $\mathrm{CHO}$ and BHK-21 cells, with a stronger effect of the dominant-negative form than the constitutively active form (Ullrich et al., 1996). Rab11a ${ }^{525 N}$ has also been expressed in mouse oocytes. The first effect observed was a loss of transferrin transport (Schuh, 2013). It was then observed that the actin network became static, and the meiotic spindle was unable to migrate to the cortex, which was specific compared to dominant-negative variants of other Rab proteins (Holubcová et al., 2013). Thus, Rab11a ${ }^{\mathrm{S} 25 \mathrm{~N}}$ expression is an effective method to specifically perturb Rab11a function in cells.

\section{Formin and Spire actin nucleators}

As described above, the first step of actin filament polymerisation is nucleation, forming a complex of actin monomers. Different actin nucleators and actin-binding proteins can affect the rate, dynamics and direction of actin polymerisation. As Formin-2 and Spire1/2 actin nucleators have been found to co-localise with Rab11a in oocytes and modulate the actin cytoskeleton (Holubcová et al., 2013), they formed a part of the investigations described in this thesis.

Formins are a family of highly conserved proteins that have a prominent role in actin cytoskeleton organisation. Formins assemble into donut-shaped dimers, each subunit being able to bind two actin monomers for nucleation. They have two important domains; FH1 and FH2. FH1 contains a prolinerich region and promotes elongation by enabling the delivery of G-actin to the barbed end of the filament, while $\mathrm{FH} 2$ is a homodimeric domain at the $\mathrm{C}$-terminus of Formin proteins that stabilises actin dimers at nucleation (reviewed by Chesarone et al., 2010; Firat-Karalar and Welch, 2011). The first Formin gene to be discovered was Fmn1, located in the limb deformity locus, as its mutation leads to a failure in limb development in mice (Woychik et al., 1985, 1990). Since then, several Formin-related proteins have been found in a large variety of species. Notably, in Drosophila, cappuccino was described to be a maternal-effect fertility factor, as females with a mutation produced embryos with disorganised cellular structures and incorrect polarities (Emmons et al., 1995; Manseau and Schüpbach, 1989). A novel mammalian member of the cappuccino subfamily was subsequently found and named Formin-2. Formin-2 is a 1578-amino-acid-long, $167 \mathrm{kDa}$ heavy protein. Its FH1 domain has a particularly long proline motif compared to other Formins, and unlike Formin-1, appears to be expressed only in a single splice form. Furthermore, using in situ hybridisation, Fmn2 was found to be expressed in the mouse embryo brain (Leader and Leder, 2000). However, a knock-out of Fmn2 found no clear brain defects; rather, $\mathrm{Fmn}^{-/-}$female mice were found to be subfertile, suffering from recurrent pregnancy loss and low litter size of only 1-3 pups, if any. Closer inspection revealed gross deformations of embryos, as well as embryo reabsorption in the uterine decidua. As ovary transplants 
rescued this phenotype, the oocytes of $\mathrm{Fmn}^{-/}$mice were next investigated, and it was discovered that meiotic spindles in $\mathrm{MI}$ oocytes fail to position to the cortex, resulting in $\mathrm{MI}$ arrest or polyploid embryos (Leader et al., 2002). Subsequent studies revealed the that function of Formin-2 in the assembly of the dynamic actin mesh in mouse oocytes is to allow spindle migration to the cortex, as described above (Dumont et al., 2007a; Holubcová et al., 2013; Pfender et al., 2011; Schuh and Ellenberg, 2008). However, the function of Formin-2 in mouse zygotes had not been studied.

Spire proteins were originally discovered in Drosophila, and were the first members of a new, third category of actin nucleators; the tandem-monomer-binding nucleators (Quinlan et al., 2005). This group of nucleators bind and bring together G-actin monomers, driving actin filament nucleation through their $\mathrm{WH} 2$ domains, and has since been found to contain other members such as APC and leiomodin (reviewed by Firat-Karalar and Welch, 2011). Spire proteins contain a KIND domain and a cluster of four WH2 domains at their N-terminal (Bosch et al., 2007); the WH2 domain cluster is critical to allow high rates of actin nucleation, as it allows tight and stable binding of four actin monomers at a time (Ducka et al., 2010). In Drosophila, Spire and the Formin Cappuccino are required for establishing the dorsal-ventral and anterior-posterior axes in eggs and embryo (Manseau and Schüpbach, 1989); together, they assemble a cytoplasmic actin mesh (Dahlgaard et al., 2007). Two mammalian homologs of Spire proteins have been found, Spire1 and Spire2. Spire1 has a length of 598 amino acids, with an atomic mass of $68 \mathrm{kDa}$, while Spire2 is slightly larger, containing 718 amino acids and weighing $80 \mathrm{kDa}$. Using in situ hybridisation of mouse embryos, it was found that Spire1 showed a similar expression pattern to Fmn2 in various brain regions (Schumacher et al., 2004). Similar to Formin proteins, despite this localisation, a function for Spire proteins in mouse brain has not been described. However, siRNA of Spire1 and Spire2 revealed a functional role in oocyte spindle migration and polar body extrusion through regulation of the actin cytoskeleton together with Formin-2. While siRNA of Spire1 or Spire2 individually did not result in any obvious phenotype, co-inhibition of both caused a reduction of actin filaments in the cytoplasm, as well as defects in asymmetric spindle positioning and polar body extrusion, indicating that these two proteins act redundantly in oocytes. Furthermore, overexpression of Spire1, Spire2, and Fmn2 mRNA together showed a greater increase in actin assembly than individual overexpression combined, leading to the conclusion that these actin nucleators act synergistically as a functional unit to nucleate the actin cytoskeleton (Pfender et al., 2011). In HeLa cells, Formin and Spire actin nucleators have not only been shown to interact together in the cytoplasm, but also to assemble nuclear actin filaments in response to DNA damage (Belin et al., 2015). Unfortunately, the large size of Formin-2 has made analysis of its exact structural properties difficult, but crystal structures of partial domains has made it clear that Spire and Formin proteins interact directly at the Spire protein KIND domain, and at the FH2 domain of Formin-2 (Vizcarra et al., 
2011; Zeth et al., 2011). Finally, a more recent study showed the precise mechanism of Spire and Formin interaction in actin assembly in vitro. Spire proteins cap barbed ends of actin, recruiting Formin-2, which in turn releases the Spire protein. This leads to what was described as a "ping-pong"like procession, as Spire and Formin-2 continue to replace and recruit each other at the barbed end. Inhibition of this interaction in vivo by injecting the purified FH2 domain into mouse oocytes inhibited actin assembly (Montaville et al., 2014). Thus, the interaction of Spire and Formin actin nucleators in regulating actin assembly in mouse oocytes has been well studied, although no such studies so far existed in mouse zygotes.

In the mouse, Spire1/2 and Formin-2 have been inhibited in two ways; by genetic knock-out for Fmn2, (Leader et al., 2002), and by siRNA for both (Pfender et al., 2011). However, as our protocol for zygote imaging works best with superovulated mice from a hybrid mouse strain (see Methods for details), neither of these options are feasible; siRNA in oocytes requires oocytes that have been matured in vitro from the early follicular stage (Pfender et al., 2015). This, and the fact that a knock-down of Spire $1 / 2$ results in issues in spindle migration and cytokinesis of the polar body, means that such oocytes would not be well-suited for IVF. Similarly, the $\mathrm{Fmn}^{-/}$mouse strain is subfertile, as asymmetric division of oocytes is perturbed (Leader et al., 2002). Therefore, this system requires an acute method of perturbing Spire1/2 and Formin-2 function in zygotes, after spindle migration and polar body extrusion have occurred successfully. As mentioned, injecting the purified $\mathrm{FH} 2$ domain competitively inhibits interaction of Spire1/2 and Formin-2 at their KIND/FH2 domains, and this has been shown to thereby decrease actin nucleation in oocytes (Montaville et al., 2014). Thus, injection of purified $\mathrm{FH} 2$ is a plausible method to perturb Spire-Formin interactions in mouse zygotes. 


\subsection{Scope of thesis and objectives}

The focus of this project has been to investigate the mechanisms of pronuclear migration in mouse zygotes, with a specific focus on the early, fast migration of male pronuclei away from the cortex. The methods initially established by Kathleen Scheffler were used and optimised to investigate the localisation and function of Rab11a, Spire2, and Formin-2. The dominant-negative Rab11a ${ }^{\mathrm{S} 25 \mathrm{~N}}$ was first used to test the function of Rab11a specifically, as opposed to brefeldin A sensitive vesicles generally (Results section 3.2). Experimental set-ups were then newly optimised and/or fine-tuned to allow observation and quantification of Rab11a localisation throughout zygotic development, both in live and in fixed cells (Results section 3.3). High temporospatial resolution was optimised to investigate the localisation of fluorescently labelled Rab11a, Spire2, and Formin-2 at the forming male pronucleus (Results section 3.4). The function of Spire2 and Formin-2 actin nucleation was tested by investigating pronuclear migration and localisation of actin nucleators in the presence of purified $\mathrm{FH} 2$, as well as Spire2 overexpression (Results section 3.5). As the nature of this fast migration was of interest, the next set of experiments investigated whether the sperm entry site or fertilisation cone were relevant to this fast migration. Female pronuclei were induced to form at the cortex with nocodazole treatment, and the localisation of fluorescently labelled Rab11a and Spire2 was analysed. Actomyosin contractions of the fertilisation cone were also perturbed with the MLCK inhibitor ML-7, and pronuclear migration as well as actin nucleator localisation as a result of this perturbation were scored (Results section 3.6). Finally, to distinguish whether actin was important only for this fast, early migration, or also important later, the effect of acute cytochalasin D treatment on pronuclear migration after the fast migration was investigated (Results section 3.7). These experiments, along with further experiments carried out by Kathleen Scheffler on the slower migration in later stages of the zygote, will be taken together to summarise our new findings about the mechanisms of pronuclear migration in mouse zygotes. The discussion section will consider and review these results in the context of the current state of knowledge. 
Mechanisms of pronuclear migration in mammalian zygotes: Introduction

This page is intentionally left blank 


\section{MATERIALS \& METHODS}

\subsection{Molecular biology}

\section{Cloning of new expression constructs for live imaging in mouse zygotes}

New constructs for mRNA synthesis and expression in oocytes and zygotes were cloned as part of this project; I subcloned pGEMHE-mScarlet-Rab11a and pGEMHE-mClover3-Spire2, changing the fluorophore from existing plasmids (Pfender et al., 2011; Schuh, 2013), and also replaced the backbone of the commercial Actin-VHH-TagGFP chromobody (Chromotek) with the pGEMHE backbone (Liman et al., 1992) for high expression mRNA synthesis. Standard molecular biology protocols were used (Green and Sambrook, 2012). All primers used for PCR reactions and sequencing are shown in Table 2.

Table 2: Primer sequences used in this study. Primer name and sequence. All oligos were ordered from Sigma, and sequencing was done by Microsynth Seqlab. F=Forward, $R=$ Reverse.

\begin{tabular}{|l|l|}
\hline Primer name & Primer sequence \\
\hline mScarlet-Rab11a-GIBSON-F & GCTGTACAAGCTCGAGCTCAGCATGGGCACCCGCGACGAC \\
\hline mScarlet-Rab11a-GIBSON-R & CCGTCGACTGCAGAATTCGATTAGATGTTCTGACAGCACTGCACCTTTG \\
\hline mClover3-F & TATAGCTAGCGCCACCATGGTGAG \\
\hline mClover3-R & TATACTCGAGACTTGTACAGCTCGTCCATG \\
\hline CMV promoter-F & CGCAAATGGGCGGTAGGCGTG \\
\hline SV40-R & CCTCTACAAATGTGGTATGG \\
\hline Actin-VHH-TagGFP-GIBSON-F & GGTCTCAGATCTCGAGCTCAATGGCTCAGGTGCAGCTG \\
\hline Actin-VHH-TagGFP-GIBSON-R & CCGTCGACTGCAGAATTCGATTACCTGTACAGCTCGTCC \\
\hline pGEMHE sequencing-F & AAGGCGATTAAGTTGGGTAACG \\
\hline pGEMHE sequencing-R & TCGGGTGTTCTTGAGGCTGG \\
\hline
\end{tabular}

All PCR reactions were set up using the Phusion polymerase kit (NEB), in a $50 \mu \mathrm{L}$ reaction with $1 \mathrm{X}$ Phusion buffer, $200 \mu \mathrm{M}$ dNTPs, $500 \mathrm{nM}$ of each forward and reverse primers, $100 \mathrm{ng}$ plasmid DNA, and $0.5 \mu \mathrm{L}$ (1 unit) Phusion DNA Polymerase. The PCR program was run with following settings: Initial denaturation $95^{\circ} \mathrm{C} 3$ minutes; $40 \mathrm{x}$ cycle of denaturation $95^{\circ} \mathrm{C} 30$ seconds, variable annealing temperatures $\left(64.5-68^{\circ} \mathrm{C}\right) 30$ seconds, extension $72^{\circ} \mathrm{C} 30$ seconds; final extension $72^{\circ} \mathrm{C} 5$ minutes; $10^{\circ} \mathrm{C}$ hold.

For pGEMHE-mScarlet-Rab11a, GIBSON assembly (Gibson et al., 2009) was used. Primers (mScarletRab11a-GIBSON-F and -R) were designed using the NEBuilder (NEB) to synthesise a PCR product of Rab11a. The PCR reaction was set up as above with mScarlet-Rab11a-GIBSON-Forward and -Reverse 
primers and pGEMHE-mCherry-Rab11a (Schuh, 2013) as DNA source, with an annealing temperature of $68^{\circ} \mathrm{C}$. In parallel, a digest of $p G E M H E-m S c a r l e t$ vector (cloned by Shiya Cheng in the lab from Addgene plasmids) $(2 \mu \mathrm{g})$ with HindIII-HF (NEB) in a $50 \mu \mathrm{L}$ reaction with CutSmart buffer (NEB) was set up for 1 hour at $37^{\circ} \mathrm{C}$. PCR product and digest were run on a $1 \%$ agarose (Sigma)/TBE gel for 25 minutes, and bands cut out and eluted with Gel Extraction kit (QIAGEN). The GIBSON assembly mixture was set up using a 1:3 vector:insert ratio and at least $100 \mathrm{ng}$ of vector DNA, with $10 \mu \mathrm{L}$ GIBSON Assembly Master mix (NEB) in $20 \mu \mathrm{L}$ total volume, incubated at $50^{\circ} \mathrm{C}$ for 1 hour.

For $p G E M H E-m C l o v e r 3-S p i r e 2, m C l o v e r 3$ was amplified using PCR from the $p G E M H E-m C l o v e r 3$ vector (cloned by Shiya Cheng in the lab from Addgene plasmids) with mClover3-F and -R primers and an annealing temperature of $68^{\circ} \mathrm{C}$, followed by digestion with Nhel-HF and Xhol (both NEB) in a $50 \mu \mathrm{L}$ reaction with CutSmart buffer (NEB) for 1 hour at $37^{\circ} \mathrm{C}$. The $m$ Cherry sequence was cut out from the plasmid pGEMHE-mCherry-Spire2 (Pfender et al., 2011) using Nhel-HF and Xhol in a $50 \mu \mathrm{L}$ reaction with CutSmart buffer (NEB) for 1 hour at $37^{\circ} \mathrm{C}$, followed by 1 hour of CIP treatment (NEB). Next, the digested mClover3 PCR product and pGEMHE-Spire2 vector were run on a $1 \%$ agarose/TBE gel, and the correct bands were eluted from the gel using a Gel Extraction kit (QIAGEN). Ligation was set up using a 1:3 vector:insert ratio and at least $100 \mathrm{ng}$ of DNA of vector DNA, with $2 \mu \mathrm{L}$ ligase buffer and $1 \mu \mathrm{L}$ T4 ligase (NEB) in a $20 \mu \mathrm{L}$ reaction for 1 hour at $37^{\circ} \mathrm{C}$. A vector-only control ligation mix was also set up with the same conditions.

The Actin-VHH-TagGFP chromobody plasmid was purchased from Chromotek. However, as the full backbone sequence was not available, the original plasmid was sent for sequencing using the standard CMV promoter forward primer, and SV40 reverse primer (Seqlab). The resulting sequence was used in the NEBuilder tool to design primers (Actin-VHH-TagGFP-GIBSON-F and -R) for GIBSON assembly of the entire coding region into the pGEMHE vector. The PCR reaction was set up using Actin-VHHTagGFP-GIBSON-F and -R primers, and the purchased Actin-VHH-TagGFP plasmid, with an annealing temperature of $64.5^{\circ} \mathrm{C}$. In parallel, a digest of the pGEMHE vector $(2.5 \mu \mathrm{g})$ with HindIII-HF (NEB) in a $50 \mu \mathrm{L}$ reaction with CutSmart buffer (NEB) was set up for 1 hour at $37^{\circ} \mathrm{C}$. PCR product and digest were run on a $1 \%$ agarose/TBE gel for 25 minutes, and bands cut out and eluted with Gel Extraction kit (QIAGEN). Note that other bands were also found for PCR product, as we did not know the full background sequence, but the correct band size (1200 bp) was cut out and eluted. The GIBSON assembly mixture was set up using a 1:3 vector:insert ratio and at least $100 \mathrm{ng}$ of DNA of vector DNA, with $10 \mu \mathrm{L}$ GIBSON Assembly Master mix (NEB) in $20 \mu \mathrm{L}$ total volume, incubated at $50^{\circ} \mathrm{C}$ for 1 hour.

All resulting plasmids were transformed into STELLAR competent cells (Clontech), the cells plated and cultured overnight in LB medium (homemade) with $100 \mu \mathrm{g} / \mathrm{mL}$ Ampicillin (Sigma). Several colonies 
were picked for each plasmid, followed by an overnight culture, miniprep (QIAGEN), and sequencing of the clones (Seqlab) using forward and reverse primers for pGEMHE to sequence the coding region. Following confirmation of correct cloning by sequencing, $200 \mathrm{~mL}$ bacterial inoculations were set up, and a Midiprep was done using a kit suited for subsequent in vitro transcription (PureLink, Invitrogen).

\section{mRNA synthesis}

mRNA was synthesised in vitro for later injection into oocytes and zygotes. A list of mRNA constructs used in this study are shown in Table 3. mRNA synthesis was performed using the HiScribe T7 ARCA kit (NEB), following manufacturer's instructions. In short: All bench spaces and equipment were kept RNAse-free. Plasmid DNA (10-15 $\mu \mathrm{g})$ was linearised with $5 \mu \mathrm{L}$ Ascl restriction enzyme (NEB) and $1 \mathrm{X}$ CutSmart Buffer (NEB) in $200 \mu \mathrm{L}$ total volume at $37^{\circ} \mathrm{C}$ for 1-2 hours. Linearised DNA was extracted using phenol/chloroform and precipitated in $100 \%$ ethanol with $80 \mathrm{mM}$ sodium acetate. After centrifugation at $4^{\circ} \mathrm{C}$, pellet was washed in $70 \%$ ethanol, re-centrifuged, and dried. The pellet was resuspended in $6 \mu \mathrm{L}$ RNAse free $\mathrm{H}_{2} \mathrm{O}$ and concentration measured by Nanodrop. A $40 \mu \mathrm{L}$ reaction mix using HiScribe T7 ARCA mRNA kit (NEB) was set up according to kit instructions using $4 \mu \mathrm{g}$ of linearised DNA; incubated for 4 hours at $40^{\circ} \mathrm{C}$. TURBO DNAse (Thermo Fisher) treatment was subsequently performed at $37^{\circ} \mathrm{C}$, mRNA was PolyA tailed (NEB) for 30 minutes at $37^{\circ} \mathrm{C}$ and mRNA was cleaned up using NucleoSpin RNA Clean-Up kit (Macherey-Nagel). Uncapped RNA was dephosphorylated using Antarctic phosphatase (NEB) at $37^{\circ} \mathrm{C}$ for 30 minutes. The mRNA was extracted using chloropane and precipitated in $100 \%$ ethanol at $-80^{\circ} \mathrm{C}$ overnight. After centrifugation at $4{ }^{\circ} \mathrm{C}, 70 \%$ ethanol wash, and re-centrifugation, the pellet was completely dried and re-suspended in $11 \mu \mathrm{L}$ RNAse free $\mathrm{H}_{2} \mathrm{O}$. Concentration was measured using a Nanodrop ${ }^{\mathrm{TM}}$ (Thermo Scientific). 
Table 3: mRNA constructs used in this study. mRNA was synthesised from plasmids. Table shows name of mRNA; their use in experiments (for live imaging of a structure of interest and/or overexpression/perturbation); and end concentration of mRNA used for microinjection into oocytes. All constructs were in pGEMHE vector backbones.

\begin{tabular}{|l|l|l|}
\hline mRNA name & Structure/use & End concentration injection (ng/ $\mu \mathrm{L})$ \\
\hline H2B-mCherry & Chromosomes & 10 \\
\hline H2B-mEGFP & Chromosomes & 20 \\
\hline MyrGFP & Membrane & $150-300$ \\
\hline SNAP-Rab11a ${ }^{\text {S25N }}$ & Dominant negative & 300 \\
\hline SNAP-Rab11aWT & Control for Rab11a ${ }^{\text {S25N }}$ & 300 \\
\hline mScarlet-Rab11a & Rab11a + vesicles & For tests: 150-400; Final: 300 \\
\hline EGFP-Rab11a & Rab11a + vesicles & 300 \\
\hline mEGFP-Spire2 & Spire2 & Low: 150; High: 400; Very High: 1200 \\
\hline mCherry-Spire2 & Spire2 & Low: 150; High: 400 \\
\hline mClover3-Spire2 & Spire2 & 150 \\
\hline Formin-2-EGFP & Formin-2 & 1000 \\
\hline EGFP-UtrCH & F-actin & $200-800$ \\
\hline mClover3-UtrCH & F-actin & 400 \\
\hline Actin-VHH-TagGFP & Actin chromobody & 2000 \\
\hline
\end{tabular}




\subsection{Mouse oocyte and zygote experiments}

\section{Animals used}

Mice were maintained and handled in the MPI-BPC animal facility (BTL) according to the standards of the Federation for Laboratory Animal Science Associations (FELASA) and the German national authorities, in a pathogen-free environment. For all superovulation experiments, 8-12-week-old wildtype C57BL/6J x CBA/CaOlaHsd F1 mice were used. For some test experiments, GV oocytes were also used from this and the $129 /$ Sv strain.

\section{GV oocyte isolation}

GV oocytes were collected for a number of test experiments as described previously (Schuh and Ellenberg, 2007). To retrieve oocytes, 8-12-week-old mice were sacrificed by $\mathrm{CO}_{2}$ and/or cervical dislocation, and ovaries were dissected and collected in HEPES-buffered M2 medium containing $250 \mu \mathrm{M}$ dbcAMP (Sigma) to block meiotic progression of oocytes. Ovaries were punctured with hypodermic needles to release GV oocytes, which were stripped of cumulus cells.

In the case of Formin-2 antibody tests, GV oocytes were collected from 129/Sv wild-type and Formin2 knock-out mice (Leader et al., 2002) and fixed. For Spire1/2 and Rab11a antibody tests, GV oocytes were collected from $\mathrm{C} 57 \mathrm{BL} / 6 \mathrm{~J} \times \mathrm{CBA} / \mathrm{CaOlaHsd} \mathrm{F} 1$ mice, washed through nine drops and incubated in either methanol (control) or brefeldin A for one hour before fixation. To test actin probes for live imaging, GV oocytes were collected from C57BL/6J x CBA/CaOlaHsd F1 mice and injected with actin probe of interest. For ML-7 co-treatment tests, GV oocytes were collected from C57BL/6J $x$ $\mathrm{CBA} / \mathrm{CaO}$ laHsd F1 mice, washed through four drops and incubated in medium containing ML-7.

\section{Superovulation and IVF experiments}

Superovulation and IVF experiment conditions were optimised and established by Kathleen Scheffler (unpublished). 8-12-week-old C57BL/6J x CBA/CaOlaHsd F1 females were injected with 7.5 IU pregnant mare serum gonadotropin (PMSG, THP Medical Products) followed by 7.5 IU human chorionic gonadotropin (hCG, Intervet) 48 hours later. After 13 hours, mice were sacrificed and their oviducts carefully dissected and transferred into HEPES-buffered MEM- $\alpha$ medium (Gibco) with $5 \%$ foetal bovine serum (FBS, Gibco), 4 mM NaHCO3 (Sigma), 20 mM HEPES (Gibco), 0.075 g/L Potassium Penicillin-G (Sigma) and $0.05 \mathrm{~g} / \mathrm{L}$ Streptomycin Sulphate (Sigma). MII oocytes were collected from oviducts with a hypodermic needle.

Unless otherwise stated, culture dishes were prepared by covering droplets of medium in $35 \mathrm{~mm}$ culture dishes (Falcon) with paraffin oil (Merck Millipore), and equilibrating the dishes prior to use. When only temperature needed to equilibrate, dishes were prepared and incubated at $37^{\circ} \mathrm{C}$ 
15 minutes prior to use; in the case of $\mathrm{CO}_{2}$-dependent medium, dishes were equilibrated overnight at $37^{\circ} \mathrm{C}, 5 \% \mathrm{CO} 2$, ambient oxygen.

MIl oocytes were cultured and microinjected in HEPES-buffered MEM- $\alpha$ medium. After microinjection, MII oocytes were washed through 9 droplets and incubated in $\mathrm{CO}_{2}$-dependent MEM- $\alpha$ medium with 5\% FBS, 26 mM NaHCO3, 0.075 g/L Potassium Penicillin-G, and 0.05 g/L Streptomycin Sulphate, for mRNA expression.

Sperm was collected from $\mathrm{C} 57 \mathrm{BL} / 6 \mathrm{~J} \times \mathrm{CBA} / \mathrm{CaOlaHsd}$ male mice (over 12 weeks old). Sperm was capacitated for two hours in HTF medium (Millipore) with $2 \mathrm{mM}$ Hypotaurine (Sigma), in four-well Nunc dishes (Thermo Fisher) in a humidified chamber. MII oocytes were subsequently washed into $40 \mu \mathrm{L}$ droplets of HTF medium, and in vitro fertilisation (IVF) was performed by adding 2-5 $\mu \mathrm{L}$ of capacitated sperm to the droplets. After two hours of IVF, zygotes were washed through nine droplets of HEPES-buffered M2 medium, and prepared for live-cell microscopy or immunofluorescence.

\section{Microinjection of mRNA and protein}

Microinjection was performed using the same set-up as described previously (Jaffe and Terasaki, 2004; Schuh and Ellenberg, 2007), into either MII oocytes or zygotes as described below.

In the case of fluorescently tagged proteins visualised in live imaging as well as expression of dominant-negative or wild-type proteins, mRNA mixes were prepared to yield desired end concentrations for each mRNA as listed in Table 3. Microinjections were conducted on a Zeiss Axiovert A1 inverted microscope with Narishige MO-202U micromanipulators, using an oil pump pressure system. Injection needles were pulled on a P1000 Capillary puller (Sutter Instruments) and loaded with Mercury (Sigma). Following MII oocyte retrieval from superovulated mice as described above, MII oocytes were moved into microinjection chambers filled with warm, HEPES-buffered MEM- $\alpha$ medium (see above). Approximately 5-7 pl of the desired mRNA mix was injected into the cytoplasm of each MII oocyte. After injections, oocytes were transferred to CO2-dependent MEM- $\alpha$ medium (see above) and incubated at $37^{\circ} \mathrm{C}, 5 \% \mathrm{CO} 2$, ambient oxygen, for at least 3 hours of expression before IVF.

In addition to mRNA-expressed DNA and actin markers, SiR-DNA and SiR-Actin (both Spirochrome) were used in some experiments. For SiR-DNA, zygotes were washed and imaged in M2 medium containing $250 \mathrm{nM}$ SiR-DNA, made from a $1 \mathrm{mM}$ stock in DMSO. For SiR-Actin, a $1 \mathrm{mM}$ stock was made with DMSO, and dilutions of 1:250 (4 $\mu \mathrm{M})$. 1:25 (40 $\mu \mathrm{M})$ and undiluted (1 mM) were injected directly into the cytoplasm of the oocyte.

To inhibit Formin-2/Spire2 interactions as described previously (Montaville et al., 2014), $5 \mathrm{pl}$ of 5$6 \mathrm{mg} / \mathrm{mL}$ purified FH2-6xHis protein $(51 \mathrm{kDa})$, or MBP-6xHis (43 kDa) as a control, in buffer $(20 \mathrm{mM}$ 
potassium phosphate $\mathrm{pH} 7.4,300 \mathrm{mM} \mathrm{NaCl}, 0.1 \mathrm{mM}$ EDTA and $5 \%$ sucrose) containing $0.03 \% \mathrm{NP40}$ and $5 \mu \mathrm{g} / \mathrm{mL}$ Dextran Alexa Flour 680 were injected into the cytoplasm of freshly fertilised zygotes, after the two hours of IVF were completed.

\section{Drug treatments}

For functional experiments, drugs were used to perturb function of different structures; these are listed in Table 4. After IVF, zygotes were washed through nine droplets of the M2 medium containing the respective drug or control, using separate pipette capillaries for drug and control. Depending on the experiment, zygotes were then either maintained in this medium until fixation, or placed in an imaging dish with the corresponding drug or control added to the medium.

Table 4: Drugs used for pharmaceutical inhibition in this study. Table shows structure or protein targeted by drug, vehicle control and dilution factor, final concentration used in medium, and the supplier used.

\begin{tabular}{|l|l|l|l|l|}
\hline Drug name & Structure/protein disrupted & Vehicle control (dilution) & Concentration used & Supplier \\
\hline Cytochalasin D & F-actin & DMSO (1:1000) & $5 \mu \mathrm{g} / \mathrm{mL}$ & Sigma \\
\hline Nocodazole & Microtubules & DMSO (1:1000) & $10 \mu \mathrm{M}$ & Sigma \\
\hline brefeldin A & Vesicle formation & Methanol (1:1000) & $4 \mu \mathrm{g} / \mathrm{mL}$ & Sigma \\
\hline ML-7 & MLCK & DMSO (1:333) & $30 \mu \mathrm{M}$ & Sigma \\
\hline
\end{tabular}

For nocodazole treatment, it should be noted that when nocodazole was added too soon after IVF, the maternal DNA would often fall apart into individual chromosomes and distribute, as opposed to staying together as a cluster by the spindle remnant (Deng and Li, 2009; Schatten et al., 1985) For this reason, there was a slight difference in how nocodazole experiments were set up compared to other drug experiments; after IVF, zygotes would be incubated for approximately 45 minutes to 1 hour in M2 medium prior to putting them into media containing the drug or control and onto the microscope, as opposed to washing into the drug and setting up imaging straight away after IVF.

For ML-7 treatment, culture and imaging dishes could not be covered with oil, as this would cause extraction of the drug. Therefore, zygotes were washed through four large drops of freshly-pipetted, uncovered M2 medium containing ML-7 or DMSO. Zygotes were then placed in diagonally-opposing wells of a four-well imaging dish (CellVis), filled with $400 \mu \mathrm{L} \mathrm{M2}$ medium with either ML-7 or DMSO. The remaining two wells were filled with water-soaked tissues. The zygotes were surrounded by GV oocytes, which were used to check ML-7 function by confirming polar body formation failed in the presence of ML-7. The dish was sealed with silicone grease throughout imaging. Note than ML-7 treatment was also attempted with ethanol:water (1:1) as a solvent instead of DMSO, but under this condition, zygote development was poorer. 


\section{Fixation and immunofluorescence staining}

For immunofluorescence staining, oocytes and zygotes were fixed at desired stages, e.g. at GV, MII, or zygotes at specific hours post insemination (hpi). Pre-permeabilisation solution (0.25\% Triton-X-100 (Sigma) $/ \mathrm{ddH}_{2} \mathrm{O}$ ) was used unless stated otherwise. Pre-permeabilisation and fixative solution (100 mM Hepes, $50 \mathrm{mM}$ EGTA, $10 \mathrm{mM} \mathrm{MgSO}_{4}$, 2\% Formaldehyde, 0.2\% Triton-X-100) were prewarmed to $37^{\circ} \mathrm{C}$ prior to fixation for 10 minutes. Oocytes/zygotes were placed into prepermeabilisation solution for 5 seconds, and subsequently fixed for $25-30$ minutes at $37^{\circ} \mathrm{C}$. They were then washed three times in PBT (PBS with $0.3 \%$ Triton- $\mathrm{X}$ ) and incubated overnight at $4^{\circ} \mathrm{C}$ for extraction and further permeabilisation. Cells were blocked in PBT-BSA (3-12\% BSA) for $0.5-2$ hours at room temperature, and incubated in primary antibodies either two hours at room temperature or overnight at $4^{\circ} \mathrm{C}$. They were then washed three times through PBT-BSA before being incubated in the appropriate secondary antibodies and/or dyes at room temperature for 2 hours. Lastly, oocytes and zygotes were washed three times through PBT-BSA, followed by three times through PBS for imaging. All antibody and dye solutions were diluted in PBT-BSA as listed in table $\mathbf{5}$ below. Fixed oocytes were stored long-term in PBS with $0.03 \%$ sodium azide. 
Table 5: Antibodies used for immunofluorescence. Table includes name used in this thesis (if applicable), host organism, supplier and product number, dilution used for immunofluorescence, and the exact epitope targeted or clone used, if known. Cyan: primary antibodies; Lavender: secondary antibodies; Beige: dyes and stains.

\begin{tabular}{|c|c|c|c|c|c|c|}
\hline $\begin{array}{l}\text { Target } \\
\text { protein/species }\end{array}$ & $\begin{array}{l}\text { Name in } \\
\text { thesis }\end{array}$ & $\begin{array}{l}\text { Host } \\
\text { species }\end{array}$ & Origin/Supplier & $\begin{array}{l}\text { Product } \\
\text { Number }\end{array}$ & Dilution used & $\begin{array}{l}\text { Epitope/Clone } \\
\text { (if known) }\end{array}$ \\
\hline Formin-2 & $\mathrm{Ab}-\mathrm{F} 1$ & Rabbit & Gift from Eugen Kerkhoff & N/A & $1: 50$ & N/A \\
\hline Formin-2 & $A b-F 2$ & Mouse & Santa Cruz & $\begin{array}{l}\text { Sc- } \\
376787\end{array}$ & $1: 50$ & $\begin{array}{l}\text { Human Formin- } \\
2 \text { AA520-619 }\end{array}$ \\
\hline Spire1 & $\mathrm{Ab}-\mathrm{S} 1$ & Rabbit & Gift from Eugen Kerkhoff & N/A & $1: 50$ & $\begin{array}{l}\text { Human Nup62 } \\
\text { AA24-178 }\end{array}$ \\
\hline Spire2 & $A b-S 2$ & Rabbit & Gift from Eugen Kerkhoff & N/A & $1: 50$ & N/A \\
\hline Spire1/2 & $\mathrm{Ab}-\mathrm{S3}$ & Rabbit & Gift from Eugen Kerkhoff & N/A & $1: 50$ & N/A \\
\hline Rab11 & $A b-R 1$ & Rabbit & Cell Signalling Technology & 5589 & $1: 50$ & $\begin{array}{l}\text { Human Rab11 } \\
\text { N-terminal }\end{array}$ \\
\hline Rab11a & $A b-R 2$ & Rabbit & Thermo Fisher & $71-5300$ & $1: 50$ & $\begin{array}{l}\text { Human Rab11a } \\
\text { C-terminal }\end{array}$ \\
\hline Rab11a & $A b-R 3$ & Rabbit & Abcam & Ab65200 & $\begin{array}{l}\text { For tests: } \\
\text { 1:300-1:8000 } \\
\text { End dilution: } \\
\text { 1:2000 }\end{array}$ & $\begin{array}{l}\text { Human Rab11a } \\
\text { C-terminal }\end{array}$ \\
\hline $\begin{array}{l}\alpha \text {-rabbit lgG } \\
\text { A488 }\end{array}$ & N/A & Goat & Thermo Fisher & A11034 & $1: 400$ & Rabbit IgG \\
\hline $\begin{array}{l}\alpha \text {-mouse IgG } \\
\text { A488 }\end{array}$ & N/A & Goat & Thermo Fisher & A11029 & $1: 400$ & Mouse IgG \\
\hline $\begin{array}{l}\alpha \text {-mouse IgG } \\
\text { A546 }\end{array}$ & N/A & Goat & Thermo Fisher & A11003 & $1: 400$ & Mouse IgG \\
\hline $\begin{array}{l}\text { Hoechst } 33342 \\
\text { (DNA) }\end{array}$ & N/A & N/A & Thermo Fisher & 62249 & $1: 1000$ & dsDNA \\
\hline
\end{tabular}

\section{Confocal microscopy of live and fixed cells}

Confocal imaging of live and fixed oocytes and zygotes was done using Zeiss LSM 800 or LSM 880 systems mounted with 40X water immersion objectives, incubator boxes, Piezo stage adapters and inserts, and Argon lasers. All live imaging was performed at $37^{\circ} \mathrm{C}$ and ambient oxygen/carbon dioxide, imaging of fixed samples at room temperature. Oocytes were placed in small droplets of either M2 medium (live) or PBS (fixed) covered in paraffin oil in glass-bottom dishes (MatTek), unless otherwise stated. For live imaging, fastest scanning speed and low laser settings $(<0.1 \%)$ were used, with the exception of Airyscan imaging of actin probes. For fixed cells, slower scanning speeds, a smaller pinhole and higher z-resolution were used. Exact details of z-resolution and, where relevant, time resolution, are found in the figure legends. Laser settings were adjusted to signal strength, so that the signal was neither saturated nor underexposed. 


\subsection{Image analysis, quantification and statistical analysis}

\section{D-tracking and quantitative analysis of pronuclear migration}

Analysis protocol for pronuclear migration tracking was optimised by Kathleen Scheffler (unpublished). For zygotes imaged using H2B-mCherry (DNA) and MyrGFP (membrane), cell surface and pronuclei were reconstructed in 3D using Imaris (Bitplane) software. Zygote shape was reconstructed based on MyrGFP signal of the membrane, using the Imaris Cell Module to make isosurfaces. Pronuclei position was tracked over time using the Imaris tracking function on iso-surfaces; pronuclei shape and identity were verified manually.

The distance of pronuclei from centre of the zygote were calculated using the centres of homogenous mass in Microsoft Excel. Average velocities were calculated by first calculating instantaneous velocities for each pronucleus at each time point, and then averaging these values within a specified time interval, using Microsoft Excel.

Time point of pronuclear formation for live imaging was defined as the last time point before the volume of the pronucleus increased significantly as DNA began decondensing. For the majority of experiments, this time point was set as time point 0 , with the exception of when cytochalasin $D$ or DMSO were added after onset of imaging, in which case time of drug addition was set as time point 0.

\section{Quantification of Rab11a-positive vesicles}

In live-cell experiments of zygotes expressing mScarlet-Rab11a mRNA, vesicles were counted manually in the lower half of the zygote in ZEN Black software, using the "Event Marker" tool to mark spots counted.

In fixed zygotes stained with Ab-R3, vesicles were first counted manually as above, and then counted automatically using IMARIS spot detection function. For this, a segment of $25 \times 25 \times 16.5 \mu \mathrm{m}^{3}$ around the central plane of the cell, avoiding regions overlapping with pronuclear positions, was used as a region of interest of each zygote. As the pronuclei were larger and more central in later time points $\mathrm{hpi}$, in some cases two regions of $17.73 \times 17.73 \times 16.5 \mu \mathrm{m}^{3}$ were used. Spots were detected using the region growing option of the spot detection tool, using an estimated diameter of $0.3 \mu \mathrm{m}$, but using no volume or quality threshold, thus detecting all background spots. All volumes with the identical, smallest volume of $0.0283 \mu \mathrm{m}^{3}$ were filtered out. The remaining spots were subsequently binned by size in Microsoft Excel, using bins of 1) $\left.>2 \mu \mathrm{m}^{3}, 2\right)>1 \mu \mathrm{m}^{3}$ to $\leq 2 \mu \mathrm{m}^{3}$, and 3) $>0.5 \mu \mathrm{m}^{3}$ to $\leq 1 \mu \mathrm{m}^{3}$. 


\section{Figure preparation and calculation of statistics}

For confocal images, ZEN Black and ZEN Blue were used to create Maximum Intensity Projections / Orthogonal Projections, as well as to create subset files. Images for figures were then prepared in ImageJ; contrast settings were adjusted to show areas of interest, but identical contrast settings were used for images of the same cell over time, or different cells from the same experiment. Where noted in figure legends, a gaussian blur of 1 px was applied and scale bars added.

In the results text, values are given as the arithmetic mean \pm one standard deviation, calculated in Microsoft Excel and/or Graphad/Prism. For charts, graphs, and statistical analysis, data were imported into GraphPad/Prism. Statistical analysis consisted of the following tests: Unpaired, two-tailed t-tests with Welch's correction when two groups of data were compared; One-Way ANOVA tests with Tukey's multiple comparison test for three or more groups of data; Two-tailed Fisher's exact test for contingency table $(2 \times 2)$ data. The resulting $p$-values were grouped as follows: not significant (n.s.) $p>0.05 ;{ }^{*} p \leq 0.05, * * p \leq 0.01, * * * p \leq 0.001$. 
Mechanisms of pronuclear migration in mammalian zygotes: Materials \& Methods

This page is intentionally left blank 


\section{RESULTS}

\subsection{Overview}

My project goal was to investigate mechanisms of fast pronuclear migration of the male pronucleus in mouse zygotes, with a focus on the function of Rab11a vesicles and related actin nucleators. To achieve this, I first optimised various conditions for live and fixed cell imaging, and then conducted a variety of observational and functional experiments using these methods in mouse zygotes. Here, both the troubleshooting experiments and final quantitative experiments will be shown; while the latter experiments have each been repeated at least three times with identical conditions, the former troubleshooting experiments were usually repeated only once or twice.

Due to complexity and timing of some experimental assays, and to keep number of animals used as low as possible, certain experiments were done together or in parallel with Kathleen Scheffler, which is always noted in the text and figure legends. To clearly distinguish between experiments that were solely my work and those conducted together, I will use "I" and "we" respectively in those sections.

\subsection{Rab11a is required for the early, fast migration of the male pronucleus}

In section 1.4 of the introduction, previous results from Kathleen Scheffler's experiments were shown, in which she found that brefeldin A inhibited the initial fast migration of the male pronucleus. As brefeldin A inhibits coat protein recruitment and thus vesicle formation in a variety of cell types (reviewed by Pelham B 1991), and has been shown previously to inhibit Rab11a vesicle formation and function in oocytes (Holubcová et al., 2013; Wang et al., 2008a), we wanted to test whether inhibition of Rab11a function would have the same effect as brefeldin $A$ on the fast migration of the male pronucleus. We therefore expressed a dominant-negative form of Rab11a, Rab11a ${ }^{525 N}$ fused to a SNAP domain, in mouse zygotes, and tracked their pronuclear migration using the imaging and analysis methods established previously by Kathleen Scheffler (see Introduction 1.4 and Methods 2.2). These results are shown in Figure 5.

Indeed, we found that in the presence of the dominant-negative SNAP-Rab11a ${ }^{525 N}$ protein, the male pronucleus migrated slower away from the cortex than when the wild-type SNAP-Rab11aWT was overexpressed at the same level. This can be seen in the live imaging panels shown in Figure 5A, where the cyan boxes highlight the position of the male pronuclei 30 minutes after pronuclear formation. While in the zygote expressing wild-type Rab11a, the male pronucleus formed at the cortex and quickly migrated several micrometres towards the centre, the male pronucleus in the zygote expressing dominant-negative SNAP-Rab11 $a^{S 25 N}$ mRNA formed at the cortex and was unable to migrate quickly towards the centre. Instead it grew in size while still being in close proximity to the 
cortex. At later time points, both the male and female pronuclei were able to migrate further towards the centre until nuclear envelope breakdown occurred.

This migration is quantified in Figure $\mathbf{5 B}$, where the trend described is quantitatively confirmed. The initial migration of the male pronucleus in the presence of SNAP-Rab11 $\mathrm{a}^{525 \mathrm{~N}}$ did not reach as close to the centre as in the zygotes in which the wild-type construct was expressed. In wild-type Rab11a expressing zygotes, the male pronuclei formed with a mean distance of $36.70 \pm 1.95 \mu \mathrm{m}$ from the centre; after 15 minutes, they had migrated to $28.98 \pm 3.40 \mu \mathrm{m}$ from the centre, and by 30 minutes to $24.58 \pm 2.20 \mu \mathrm{m}$ from the centre. Thus, in the first 30 minutes, they migrated an average of $12.12 \mu \mathrm{m}$. On the other hand, in the presence of SNAP-Rab11a ${ }^{525 \mathrm{~N}}$, male pronuclei formed with a mean distance of $34.95 \pm 1.83 \mu \mathrm{m}$ from the centre of the zygote; after 15 minutes, they were still $32.42 \pm 1.46 \mu \mathrm{m}$ from the centre ( $p<0.001$ compared to wild-type, Welch's t-test), and even after 30 minutes they had not migrated past $30.11 \pm 1.47 \mu \mathrm{m}$ from the centre ( $p<0.001$ compared to wild-type, Welch's t-test), a mean migration of $4.84 \mu \mathrm{m}$ in 30 minutes. At the last time point before nuclear envelope breakdown, the male pronucleus was on average $9.71 \pm 1.73 \mu \mathrm{m}$ from the centre in wildtype Rab11a expressing zygotes, and $12.88 \pm 3.61 \mu \mathrm{m}$ in SNAP-Rab11 $a^{S 25 N}$ mRNA expressing zygotes ( $p<0.001$, Welch's t-test). The female pronuclei shown on the right-hand side graph did not form as far from the centre (or close to the cortex) as the male pronuclei, whether wild-type or dominantnegative Rab11a was expressed in the zygote. In the wild-type expressing group, the female pronuclei formed with a mean distance from the centre of $23.71 \pm 2.90 \mu \mathrm{m}$, and before nuclear envelope breakdown had travelled to $10.67 \pm 2.13 \mu \mathrm{m}$ from the centre. In the dominant-negative group, the mean distance from the centre of the zygote at pronuclear formation was $23.71 \pm 2.76 \mu \mathrm{m}$, and $11.69 \pm 3.19 \mu \mathrm{m}$ at nuclear envelope breakdown, neither of which was significantly different from the wild-type.

To further analyse these data in a more quantitative manner, the average velocities in different time frames were calculated and are shown in Figure 5C. Here, the same time frames showed a significant difference between wild-type and dominant-negative Rab11a-expressing zygotes for male and female pronuclei; namely, the first 30 minutes of migration, when the average velocity was slower for both male and female pronuclei in the dominant-negative group, and the time frame from four to six hours post nuclear envelope breakdown, when the average velocity was faster in the dominant-negative group. In the first 30 minutes, the male pronucleus moved at a mean velocity of $0.40 \pm 0.08 \mu \mathrm{m} /$ minute towards the centre in the wild-type, and in the dominant-negative group the mean velocity was $0.16 \pm 0.06 \mu \mathrm{m} /$ minute $(p<0.001$, Welch's t-test); the female pronucleus moved at a mean velocity of $0.10 \pm 0.08 \mu \mathrm{m} /$ minute in the wild-type and $0.027 \pm 0.060 \mu \mathrm{m} /$ minute in the dominant-negative group ( $p<0.001$, Welch's t-test). In the time frame four to six hours post pronuclear 
formation, the male pronucleus showed an average velocity of $0.014 \pm 0.014 \mu \mathrm{m} /$ minute in the wildtype, and $0.026 \pm 0.015 \mu \mathrm{m} /$ minute in the dominant-negative group ( $p<0.001$, Welch's t-test); the female pronucleus moved $0.014 \pm 0.015 \mu \mathrm{m} /$ minute in the wild-type, and $0.023 \pm 0.019 \mu \mathrm{m} / \mathrm{minute}$ in the dominant-negative group ( $p=0.0155$, Welch's t-test). The remaining time frames did not show a significant difference between wild-type and dominant-negative Rab11a expression, neither for the male nor female pronuclei.

Overall, these results indicate a role of Rab11a in the early stages of pronuclear migration. In particular, the male pronucleus was unable to migrate as fast away from the cortex in the first 30 minutes when Rab11a function was perturbed; likely as a result of this, the male pronucleus was not as central by the time the nuclear envelope broke down as in wild-type cells. Quantification indicated that velocity of the female pronucleus was also significantly affected during the first 30 minutes, although the pronuclei were able to reach the centre as in wild-type cells by the end. During one time frame during later migration of both male and female pronuclei, the velocity was increased significantly, possibly as the pronuclei still had more distance to migrate to the centre than in wildtype-expressing zygotes by this point. Note that these experiments, which were done as part of training when I joined the project, were conducted a total of six times; one repetition of which was done by myself, the rest by Kathleen Scheffler.

As the results of this experiment indicate that not only vesicle formation in general, but specifically Rab11a has a function during the early stage of pronuclear migration, my next goal was to investigate the localisation of Rab11a in both live and immunofluorescence imaging. 
A

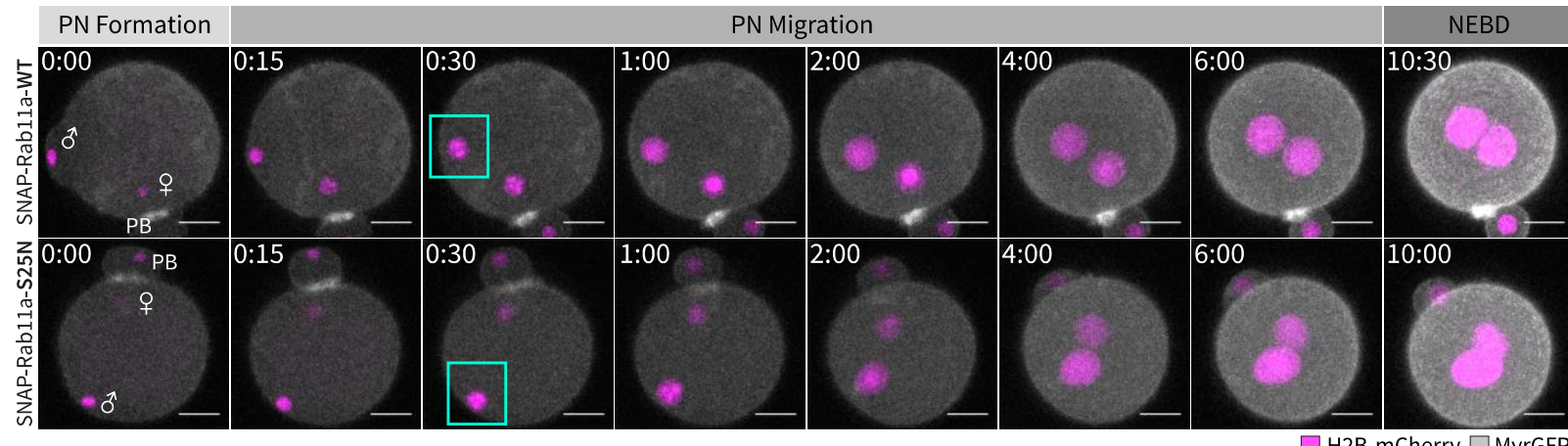

B

Migration of male pronucleus

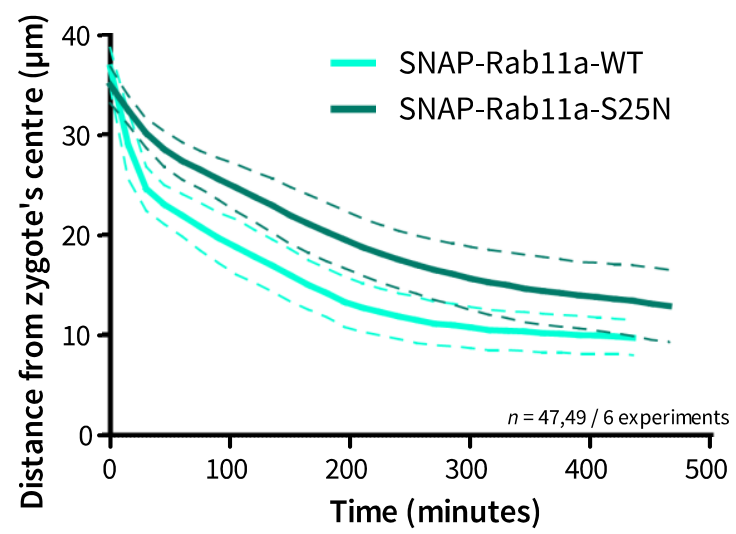

C
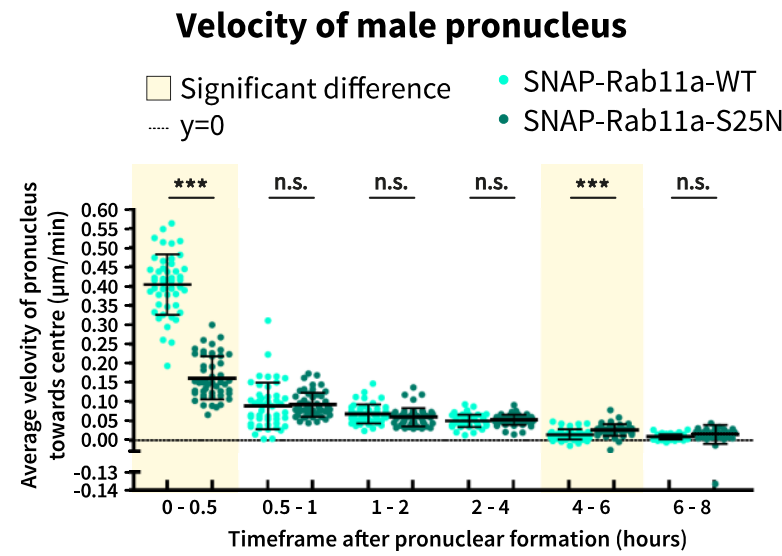

Migration of female pronucleus

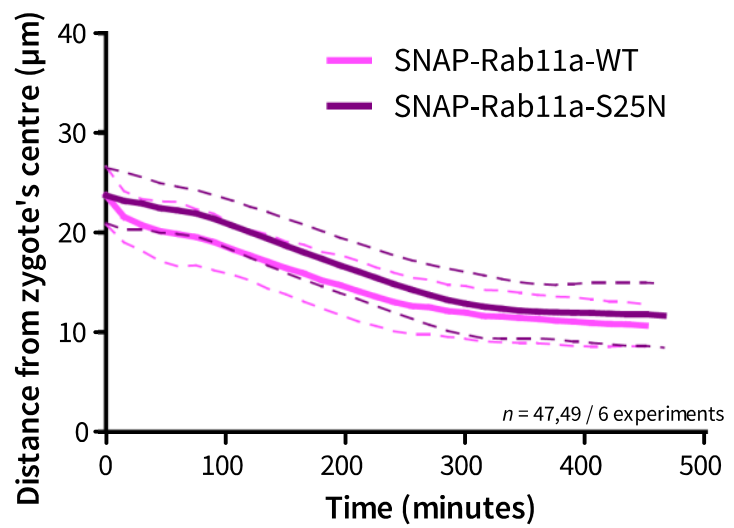

\section{Velocity of female pronucleus}

$\begin{array}{ll}\square \text { Significant difference } & \cdot \text { SNAP-Rab11a-WT } \\ \cdots-. . . & \cdot \text { SNAP-Rab11a-S25N }\end{array}$

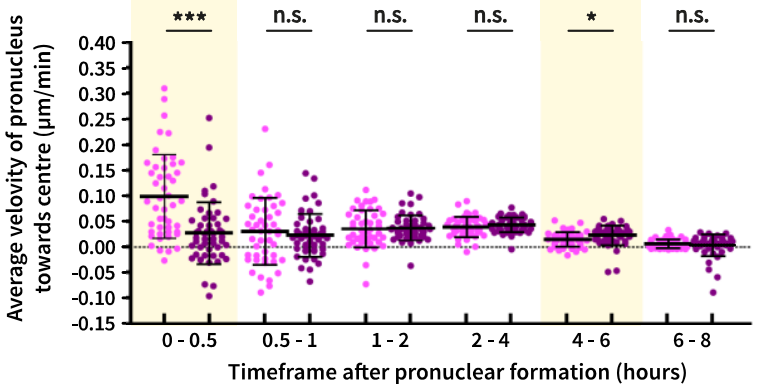

Figure 5: Rab11a is required for the early, fast migration of the male pronucleus. (A) Confocal images of live zygotes expressing wild-type SNAP-Rab11aWT or dominant-negative SNAP-Rab11a ${ }^{\mathrm{S} 25 \mathrm{~N}} \mathrm{mRNA}$, as well as MyrGFP (grey) as a membrane marker and H2B-mCherry (magenta) to visualise DNA. Cyan box highlights position of male pronucleus 30 minutes after pronuclear formation. Images taken every 15 minutes, every $3 \mu \mathrm{m}$ throughout the zygote. $P B=$ Polar body, $\sigma^{*}=$ male pronucleus $q=$ female pronucleus. Images shown are maximum intensity projections of the z-planes with a 1 px Gaussian blur filter applied. Images taken on Zeiss LSM-880 confocal microscope. Scale bar $=20 \mu \mathrm{m}$. Time shown in hours:minutes relative to pronuclear formation. Representative images; total number of zygotes $=47$ for SNAP-Rab11aWT, and 49 for SNAPRab11 $a^{525 N}$ from six experiments, where one repetition was conducted by myself, and the remaining five by Kathleen Scheffler; representative images are from my repetition. (B) Migration of female and male pronucleus over time. The live imaging data were reconstructed in $3 D$ using IMARIS software, and the relative distance of each pronucleus from the centre of the zygote was calculated and plotted over time. In the left graph, migration of male pronucleus is shown, and on the right, the female, each comparing zygotes expressing SNAP-Rab11aWT mRNA (light cyan and light magenta respectively), and SNAP-Rab11a ${ }^{\mathrm{S} 25 \mathrm{~N}}$ mRNA (dark turquoise and dark purple respectively); dashed lines = one standard deviation. (C) Velocity of male (left) and female (right) pronucleus in different time frames. The mean velocity of each pronucleus for each time frame was calculated and plotted so that each dot represents one pronucleus; the mean of all pronuclei for each time frame was calculated and is 
indicated by the black line with error bars signifying one standard deviation. The colour key is identical to (B). Note the differing $y$-axes for male and female pronuclei, and that the dotted black line shows where $y=0$. Shaded yellow area signifies time frame in which there is a significant difference between the SNAP-Rab11aWT and SNAP-Rab11aS25N groups. Statistical significance between conditions calculated with Unpaired, two-tailed t-test with Welch's correction. * $p \leq 0.05, * * p \leq 0.01, * * *$ $p \leq 0.001$. 


\subsection{Rab11a-positive vesicles are present in the early, but not the late, mouse zygote}

\section{Optimisation of Rab11a live imaging in mouse zygotes}

Before I joined the project, Kathleen Scheffler had imaged Rab11a live using EGFP-Rab11a mRNA in zygotes. Vesicles were visible, but the signal:noise ratio was not ideal; therefore, I cloned a new construct: pGEMHE-mScarlet-Rab11a, from which mScarlet-Rab11a mRNA was synthesised in vitro to be injected into and expressed in zygotes (see Methods 2.1 for cloning strategy), to see if this fluorophore would provide better images. I tested different concentrations of this new mRNA in zygotes in two preliminary experiments shown in Figure 6. MII oocytes were injected with different concentration of mScarlet-Rab11a mRNA, and imaged after fertilisation and before pronuclear formation, after a total of five hours of expression time. In the first experiment, I tested concentrations of $400 \mathrm{ng} / \mu \mathrm{L}$ and $300 \mathrm{ng} / \mu \mathrm{L}$ of the mRNA. In both zygotes shown, Rab11a-positive vesicles were clearly visible in the cytoplasm, as indicated by the cyan arrows. While setting up imaging I generally found that the Rab11a-positive vesicles were very dynamic in both number and size during this early zygotic stage. As can be seen in the images, the vesicles appeared brighter and larger in the zygote injected with $400 \mathrm{ng} / \mu \mathrm{L}$ mRNA concentration. However, as Rab11a is involved in many cellular processes, we wanted to keep overexpression to a minimum, while still allowing optimal imaging. Therefore, I decided to try even lower concentrations in the second experiment. In the images shown, brightness and contrast settings were kept the same for images taken together to allow fair comparison. Unfortunately, lower concentrations such as $200 \mathrm{ng} / \mu \mathrm{L}$ and $150 \mathrm{ng} / \mu \mathrm{L}$ made it very difficult to identify the Rab11a-positive vesicles above the noise, even with adjusted contrast settings; therefore, I decided to use the $300 \mathrm{ng} / \mu \mathrm{L}$ concentration in subsequent experiments. During these experiments, I also optimised laser power and other imaging settings such as pinhole and z-resolution to give a good signal while avoiding bleaching of the fluorescent protein, which I could then use in later experiments. For example, I first imaged the zygote every $1 \mu \mathrm{m}$ in $\mathrm{z}$ in Experiment 1 , then every $1.3 \mu \mathrm{m}$ in Experiment 2, and eventually used a z-resolution of $1.5 \mu \mathrm{m}$ for final experiments shown in later figures. 


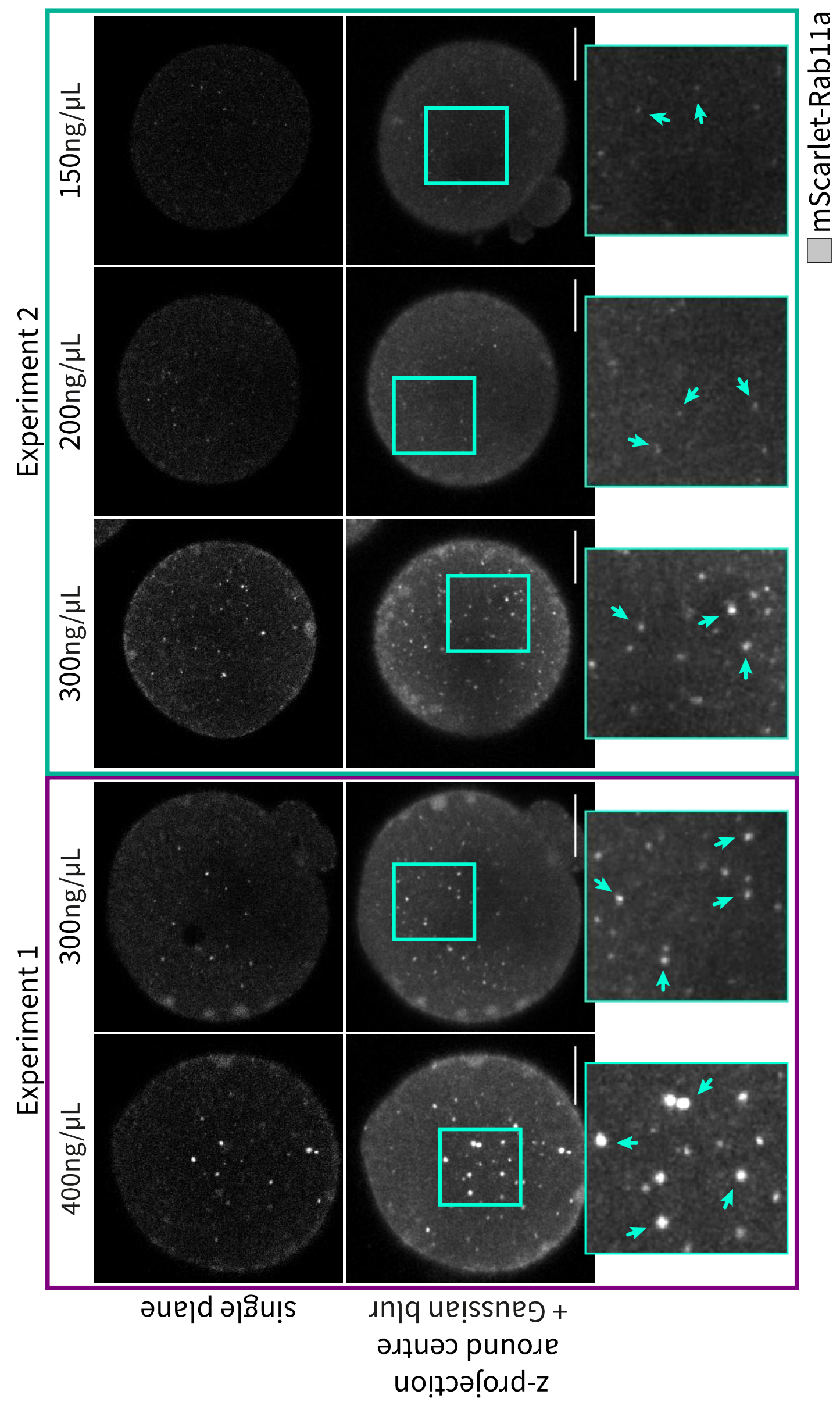

Page 61 of 177 
Figure 6: Optimisation of $m S$ carlet-Rab11a mRNA concentration and imaging conditions for live-cell imaging of mScarletRab11a. Different concentrations of mScarlet-Rab11a (grey) were expressed in two separate experiments; note that different imaging conditions such as laser power and scale were used between the two experiments, but were kept the same within each experiment. The single plane shows a central plane of the zygote, while the z-projection is a projection of 10 planes around the centre with a Gaussian blur of 1 px. In Experiment 1, one z-plane was 1 um thick, while in Experiment 2, one zslice corresponded to $1.3 \mu \mathrm{m}$. Representative images from each experiment are shown; a total of 7 zygotes were imaged per group in Experiment 1; in Experiment 2, 7 zygotes were imaged with $300 \mathrm{ng} / \mu \mathrm{L}, 5$ zygotes with $200 \mathrm{ng} / \mu \mathrm{L}$ dilution, and 4 zygotes with $150 \mathrm{ng} / \mu \mathrm{L}$ dilution. Images taken with a Zeiss LSM-800 confocal microscope. Scale bar $=20 \mu \mathrm{m}$.

\section{Live imaging of mScarlet-Rab11a in mouse zygotes}

Now that the mRNA concentration and imaging settings for live mScarlet-Rab11a imaging were optimised, mouse zygotes expressing mScarlet-Rab11a and H2B-mCherry mRNA could be imaged, to see how the presence and localisation of Rab11a-positive vesicles changed from fertilisation through pronuclear formation, migration, and up to nuclear envelope breakdown. The results from these experiments are shown in Figure 7.

Zygotes were imaged from fertilisation until nuclear envelope breakdown, with image acquisition every 15 minutes, using the mRNA and imaging conditions optimised before. The results are shown in panel Figure 7A. The cyan boxes show regions of interest in the cytoplasm or other area of the cell, shown with higher magnification below each time point. mScarlet-Rab11a-positive vesicles were seen in the cytoplasm during early time points, before pronuclear formation and at pronuclear formation, but were no longer observed in the cytoplasm after the pronucleus had formed. In some zygotes, an mScarlet-Rab11a enrichment could be seen at the cortical region adjacent to the male pronucleus just as it was forming and migrating away from the cortex, here shown at time point 00:15. This was seen in 6/12 zygotes (50\%) when imaging every 15 minutes with a z-resolution of $3 \mu \mathrm{m}$. After this early pronuclear migration, no vesicles were apparent in the cytoplasm until nuclear envelope breakdown. However, as expression levels increased and the signal became stronger and noisier, it also became more difficult to differentiate spots in the cytoplasm, and whether they were mScarlet-Rab11apositive vesicles, or simply background signal, as can be seen at the 6-and 10-hour time points.

Next, the number of vesicles over time was quantified by manual counting. The fluorescent signal was clearer and brighter closer to the objective than at the 'top' of the zygote, as at the top of the zygote, there was more cytoplasm and its lipid granules between the imaging plane and the objective. For this reason, only the half of each zygote closest to the objective was used for manual counting. The results of this manual counting can be seen in Figure 7B. A clear trend was observed; vesicles were present from fertilisation, with a mean of $42.83 \pm 14.17$ vesicles counted in the half zygote; this increased around pronuclear formation to $105.70 \pm 47.33$ vesicles, and then decreased again to $18.08 \pm 13.78$ vesicles within 30 minutes. Statistically, the increase from 30 minutes before pronuclear formation to the time point of pronuclear formation was significant $(p<0.001$, One-way Tukey's ANOVA), as was the 
drop from pronuclear formation to 30 minutes after ( $p<0.001$, One-way Tukey's ANOVA). After this time point, no statistically significant change was observed between subsequent time points anymore; only very few vesicles could be observed at the time points from 30 minutes to 5 hours. As mentioned above, the signal became stronger and noisier with time and to the human eye, no clear vesicles could be seen after 6 hours.

An additional observation that was made was that MII oocytes, in contrast to fertilised zygotes, did not show clear Rab11a-positive vesicles, as seen in Figure 7C. As the experimental focus at this point was to image zygotes, this observation was not followed up further using live imaging. However, this difference was later quantified between MII oocytes and zygotes using immunofluorescence imaging; see Figure 12.

These experiments showed that mScarlet-Rab11a-positive vesicles were present in fertilised zygotes, but not in MII oocytes, and that the number of vesicles observed peaked at the time point of pronuclear formation, and dropped to nearly zero soon after. Furthermore, in half of the zygotes imaged, an accumulation of mScarlet-Rab11a was seen adjacent to the forming male pronucleus. However, as these results were based on an overexpression of mScarlet-Rab11a, as well as manual counting for quantification, we wanted to confirm that the trend observed over time was also true of endogenous Rab11a-positive vesicles. For this, I next tested different antibodies for Rab11a, as well as for related Spire and Formin actin nucleators. 
\%
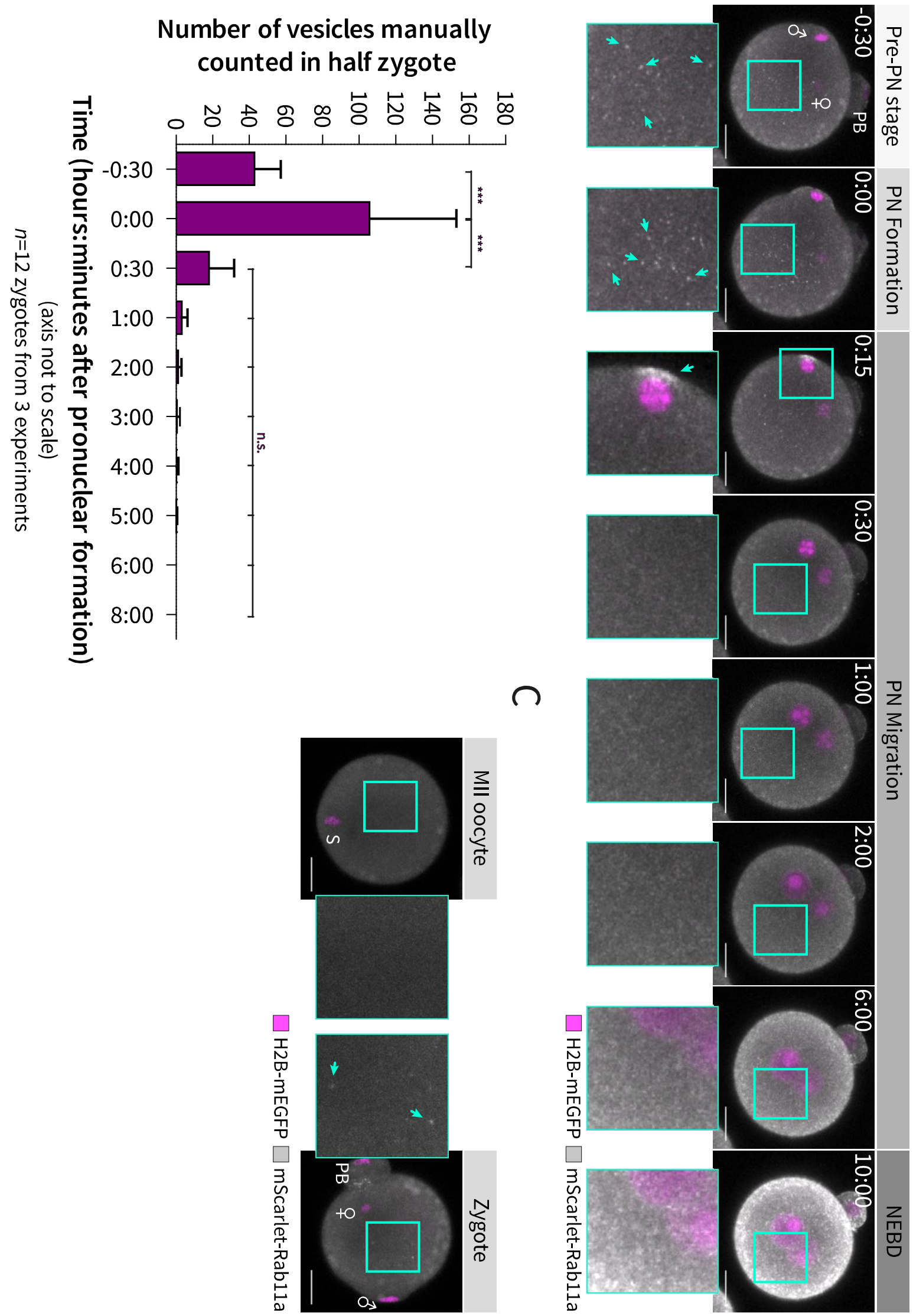
Figure 7: Live imaging of $\mathrm{mScarlet-Rab11a}$ from fertilisation to the first mitotic division. (A) Confocal images of a live, fertilised zygote expressing mScarlet-Rab11a (grey) and H2B-mEGFP (magenta) from pre-pronuclear (PN) formation stage, through PN formation (time point set as 0:00), PN migration, until nuclear envelope breakdown (NEBD); cyan boxes indicate region of interest zoomed in below each time point; cyan arrows indicate mScarlet-Rab11a-positive vesicles in cytoplasm, or in the case of 15 minutes post pronuclear formation, the accumulation of mScarlet-Rab11a in the cortical region adjacent to the forming male pronucleus. z-projection of 30x1.5 $\mu \mathrm{m}$ slices with Gaussian blur 1 px. Images taken with a Zeiss LSM-800 microscope. Scale bar $=20 \mu \mathrm{m}$. Time shown in hours:minutes relative to pronuclear formation. Representative images of a total of 12 zygotes from3 experiments are shown. (B) Number of vesicles manually counted in half zygote over time. The bottom half of each zygote was used to manually count vesicles at each shown time point relative to time point of pronuclear formation, set as 0:00. Black error bar = one standard deviation. Statistical significance calculated using One-way ANOVA with Tukey's multiple comparison test; * $p \leq 0.05, * * p \leq 0.01, * * * p \leq 0.001$. (C) Confocal images of live MIl oocyte and zygote expressing mScarlet-Rab11a (grey) and H2B-mEGFP (magenta); cyan boxes indicate region of interest zoomed in next to each zygote; cyan arrows indicate $m S$ carlet-Rab11a-positive vesicles in cytoplasm of zygote. $S=$ Spindle, $P B=$ Polar body, $\sigma^{7}=$ male pronucleus $q=$ female pronucleus. $z$-projection of $30 \times 1.5 \mu \mathrm{m}$ slices with Gaussian blur 1 px. Images taken with a Zeiss LSM800 microscope. Scale bar $=20 \mu \mathrm{m}$.

\section{Testing different antibodies for Rab11a and related Spire and Formin actin nucleators}

In the Department of Meiosis, several different antibodies against Formin-2, Spire1/2, and Rab11a/b were available; see Table 5 in the Methods section 2.2 for details. To see whether these antibodies could be useful for our experiments, I tested these antibodies in fixed GV oocytes, and for each antibody, oocytes were fixed in two different conditions; in wild-type control oocytes, and in those either lacking the protein of interest, or otherwise perturbing the function of the protein, so that a comparison would allow observation of a specific signal. In the case of Formin-2 antibody tests, oocytes were collected from mice with a $129 / \mathrm{Sv}$ background, either wild-type $\left(F m n 2^{+/+}\right)$or $F m n 2$ knock-out (Fmn2 ${ }^{-/}$) (Leader et al., 2002); a specific antibody should show a clear signal in the wild-type oocytes that is not present in the knock-out oocytes, as the target protein should not be present. In the case of Spire1/2 and Rab11a antibody tests, oocytes were collected from wild-type C57BL/J $\mathrm{x}$ CBA/J F1 mice, and incubated in either methanol or brefeldin A for one hour prior to fixation. For Spire1/2, brefeldin A has been reported to cause a decrease of Spire2-positive vesicles, and an increase of homogenous Spire2 levels in the cytoplasm of oocytes (Holubcová et al., 2013); a specific antibody would therefore be expected to stain vesicles in only the methanol-treated cells, and have an increased background in the brefeldin A-treated cells. For Rab11a, a decrease in the number of vesicles due to blocked vesicle formation has been described in oocytes treated with brefeldin $A$ (Holubcová et al., 2013). Thus, a specific antibody would be expected to allow observation of vesicles in the cytoplasm of methanol-treated oocytes, which are decreased or absent in the brefeldin Atreated oocytes. The fixation, staining and imaging protocol used can be seen in Methods section 2.2. Representative images are shown in Figure 8.

For Formin-2, two different antibodies were tested. For Ab-F1, a signal was observed throughout the cytoplasm with a very dotted or noisy pattern. There was a difference between the control oocytes and those from the $\mathrm{Fmn}_{2}{ }^{--}$mice; the noisy signal appeared darker in the knock-out oocytes, and there 
were also bright clusters in cytoplasm and around the cortex, indicated by the cyan arrows. However, no clear Formin-2-specific 'nodes' or other structures as described in live imaging of oocytes (Pfender et al., 2011) could be seen in this staining. For Ab-F2, the staining was less obvious in the cytoplasm, but very strong at the cortex. This cortical staining was decreased in $\mathrm{Fmn}^{-\%}$ oocytes, and clusters were seen in the cytoplasm, indicated by the cyan arrow in the lower Ab-F2 panels of Figure 8 . This likely indicates a cortical localisation of Formin-2 in oocytes, but no specific cytoplasmic structures could be observed. Therefore, these Formin-2 antibodies were not used any further.

In the case of Spire1/2, three different antibodies were tested. For Ab-S1, a fairly even signal was observed throughout the cytoplasm with a slightly stronger signal at the cortex, and no clear difference apparent between the methanol- and brefeldin A-treated oocytes, indicating this signal was not specific for Spire1 or Spire2. For Ab-S2 a more spot-like signal was observed throughout the cytoplasm, with no brighter signal at the cortex, and no clear difference between the methanol- and brefeldin A treated groups; possibly, the brefeldin A treated oocytes were slightly darker, but no clear Spire2-specific structures such as vesicles could be identified. For Ab-S3, there was an even signal throughout the cytoplasm, which was slightly stronger at the cortex. This was the same for methanoland brefeldin A-treated oocytes, showing no cytoplasmic increase as reported (Holubcová et al., 2013). However, in the methanol-treated group, some oocytes showed very small, weak spots in the cytoplasm (indicated by cyan arrow in upper Ab-S3 panels of Figure 8), which may correspond to Spire2-positive vesicles. However, these spots were small and hard to distinguish from the noise, and it was unclear if the signal was specific. Therefore, none of these Spire1/2 antibodies were used for further experiments.

For Rab11a, three different antibodies were tested. For Ab-R1, bright spots were detected throughout the cytoplasm in both methanol- and brefeldin A-treated samples; in fact, the spots appeared brighter in the brefeldin A-treated samples, making it unlikely that it was Rab11a vesicles that were targeted by the antibody. For Ab-R2, again bright spots were seen throughout the cytoplasm, with a slight increase at the cortex. However, no difference was seen between methanol- and brefeldin A-treated oocytes, indicating that this signal was not specific to Rab11a vesicles. For Ab-R3, there was an evenly distributed background signal throughout the cytoplasm, which was brighter at the cortex, in both methanol- and brefeldin A-treated oocytes. However, only in methanol-treated cells were small vesicle-like spots detected in the cytoplasm, indicated by the cyan arrows in the upper Ab-R3 panels in Figure 8. These spots were not present in the brefeldin A-treated oocytes, and were thus likely to correspond to endogenous Rab11a-positive vesicles. Therefore, we decided to continue optimising the conditions for this antibody, to see if it could be used to confirm our results of the live imaging. 

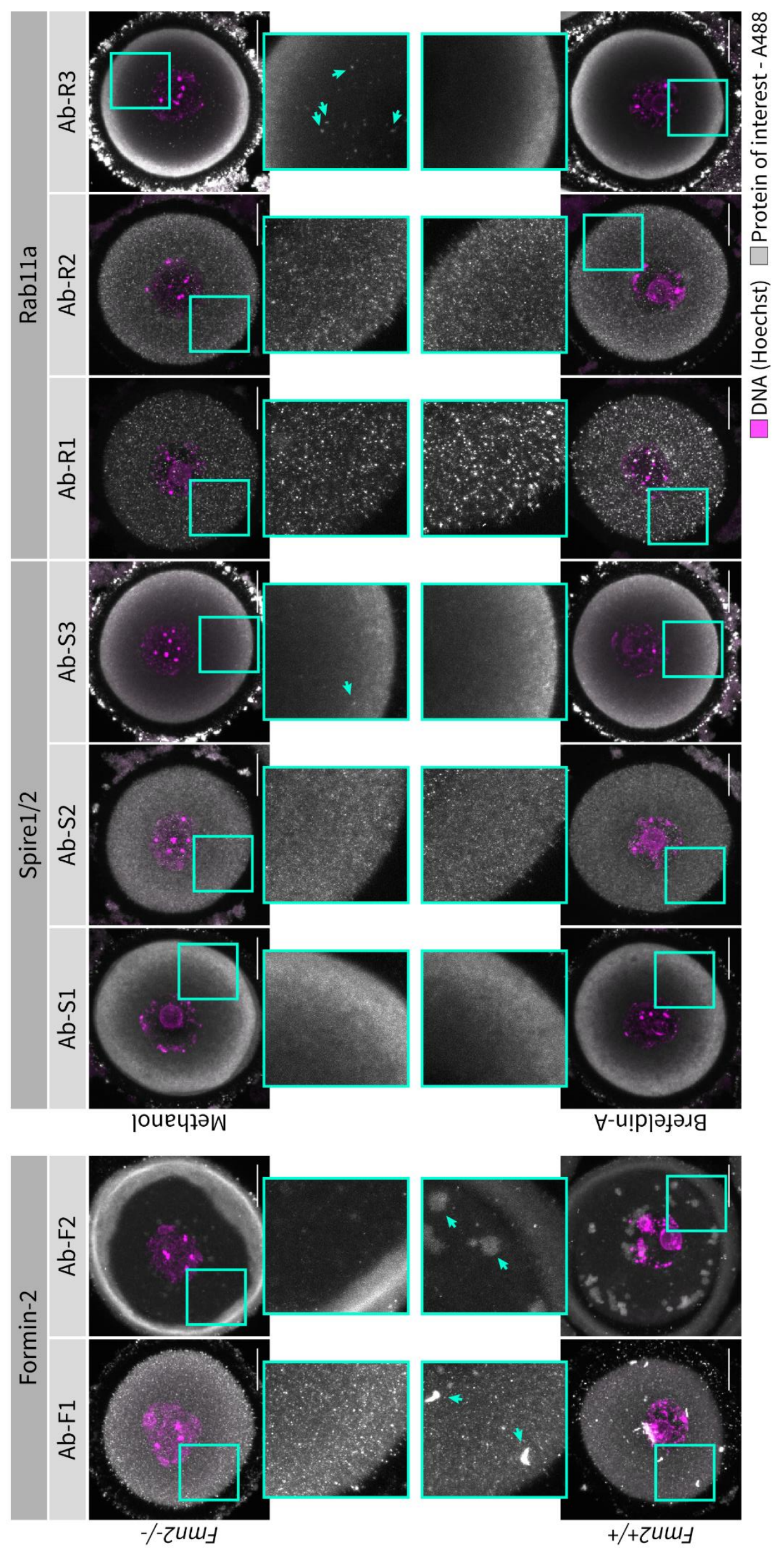

Page 67 of 177 
Figure 8: Antibody testing of Formin-2, Spire1/2, and Rab11a antibodies in GV oocytes. Confocal images of fixed GV oocytes stained with different antibodies for proteins of interest (grey) and Hoechst (DNA; magenta). For Formin-2 antibodies Ab-F1 and $\mathrm{Ab}$-F2, wild-type $\left(\mathrm{Fmn2}^{+/+}\right)$or Formin-2 knock-out (Fmn2 ${ }^{-/-}$) oocytes were used. For Spire1/2 (Ab-S1, Ab-S2, and AbS3) and Rab11a ( $A b-R 1, A b-R 2, A b-R 3)$, oocytes were incubated in either methanol or brefeldin $A$ for one hour prior to fixation. Cyan boxes indicate regions of interest amplified above/below; cyan arrows indicate structures of interest inside the oocyte. All antibodies were diluted 1:50 for these tests. Images are z-projections of $25 \times 1 \mu \mathrm{m}$ slices around the centre of the zygote. Images taken with a Zeiss LSM-880 microscope. Scale bar $=20 \mu \mathrm{m}$. Representative images are shown; a total of 10-15 zygotes were stained for each Formin-2 antibody and condition, and 3-5 oocytes were used for each Spire1/2 and Rab11a antibody and condition, though only 1-2 representative oocytes were imaged of each. Further information about the antibodies can be found in table 5.

\section{Optimising Rab11a immunofluorescence staining and imaging conditions in mouse zygotes}

As the Rab11a antibody Ab-R3 showed specific staining in GV oocytes, but also a strong cortical staining, my next goal was to optimise the conditions in mouse zygotes. The different steps of optimisation are shown in Figure 9.

The first step was to test different antibody concentrations, to see if a lower concentration would avoid the cortical staining while still allowing visualisation of the Rab11a-positive vesicles. This was done in two separate experiments, shown in Figure 9A. In the first experiment, zygotes were fixed $4 \mathrm{hpi}$, blocked in 3\% BSA in PBT, and subsequently stained with Ab-R3 dilutions ranging from 1:300 to 1:2000. The first observation was that Rab11a-positive vesicles were also observed in the cytoplasm of mouse zygotes, as in GV oocytes shown in Figure 8, indicated by the cyan arrows. The cortical staining seen in GV oocytes when using a 1:50 dilution was still present at 1:300 and 1:600 in mouse zygotes, with Rab11a-positive vesicles also visible in the cytoplasm. The cortical staining decreased in dilutions past 1:1000, with Rab11a-positive vesicles still visible in the cytoplasm. However, at these concentrations, very small spots were also visible throughout the cytoplasm, and it is unclear whether these spots correspond to smaller Rab11a-positive vesicles, or a non-specific background signal. Also note that the sperm tail, sometimes present at the zygote's cortex or in the cytoplasm, was also strongly stained with the antibody, and was therefore sometimes seen in the images.

To see if dilutions past 1:2000 improved this signal, in Experiment 2, zygotes were fixed $3.5 \mathrm{hpi}$. This was done instead of $4 \mathrm{hpi}$, as in the last experiment, it was observed that some pronuclei had already formed at $4 \mathrm{hpi}$, and for fair comparison, all zygotes should be in the pre-pronuclear stage. The zygotes were stained with $A b-R 3$ dilutions 1:2000, 1:5000, and 1:10000. While there were still bright Rab11apositive vesicles present in the 1:2000 group as in the first experiment, the 1:5000 and 1:10000 dilutions resulted in dimmer spots while still maintaining the smaller spots in the background, making it more difficult to distinguish clear vesicles. Therefore, a dilution of 1:2000 was used for subsequent experiments. 
Next, other parameters relating to the fixation, blocking and staining of the oocytes were tested, as shown in Figure 9B. For this, zygotes were fixed 3.5 hpi with or without pre-permeabilisation in $0.25 \%$ Triton-X, blocked in $3 \%, 6 \%$ or $9 \%$ BSA/PBT solution, and the secondary antibody used was either $\alpha-$ rabbit A546 or $\alpha$-rabbit A488. To cut down on conditions, the 3\% and $9 \%$ BSA groups were stained with the $\alpha$-rabbit A546 secondary antibody, while the 6\% BSA groups were stained with $\alpha$-rabbit A488. Rab11a-positive vesicles were visible in the cytoplasm of each group, but certain conditions were found to be favourable over others. First of all, the A488 fluorophore resulted in more small bright spots in the background compared to both the 3\% and 9\% BSA groups stained with A546, making the large vesicles harder to identify. For this reason, A546 was preferred as a secondary antibody fluorophore over A488. The higher BSA concentration (9\%) in the blocking solution further reduced the small spots in the background compared to the $3 \%$ solution, making the bright vesicles stand out more. The pre-permeabilisation had little obvious effect in the A546 groups, though it did reduce some background signal in the A488 group. As it did not have a negative effect, we decided to continue to use pre-permeabilisation.

The result of the final conditions used for Rab11a immunofluorescence imaging are shown in Figure 9C. This zygote was fixed $2 \mathrm{hpi}$, and is one zygote from an experiment also shown in Figure 11. As mentioned above, pre-permeabilisation was used prior to fixation, and the A546 fluorophore was used for the secondary antibody. One additional change was made compared to what was optimised in Figure $9 \mathrm{~B}$ - the $\mathrm{BSA}$ concentration in the blocking solution was further increased to $12 \%$, which resulted in an even better signal:noise ratio, as the vesicles appear very bright against an almost black background, though some small spots remain. These are the conditions used for all subsequent staining. 


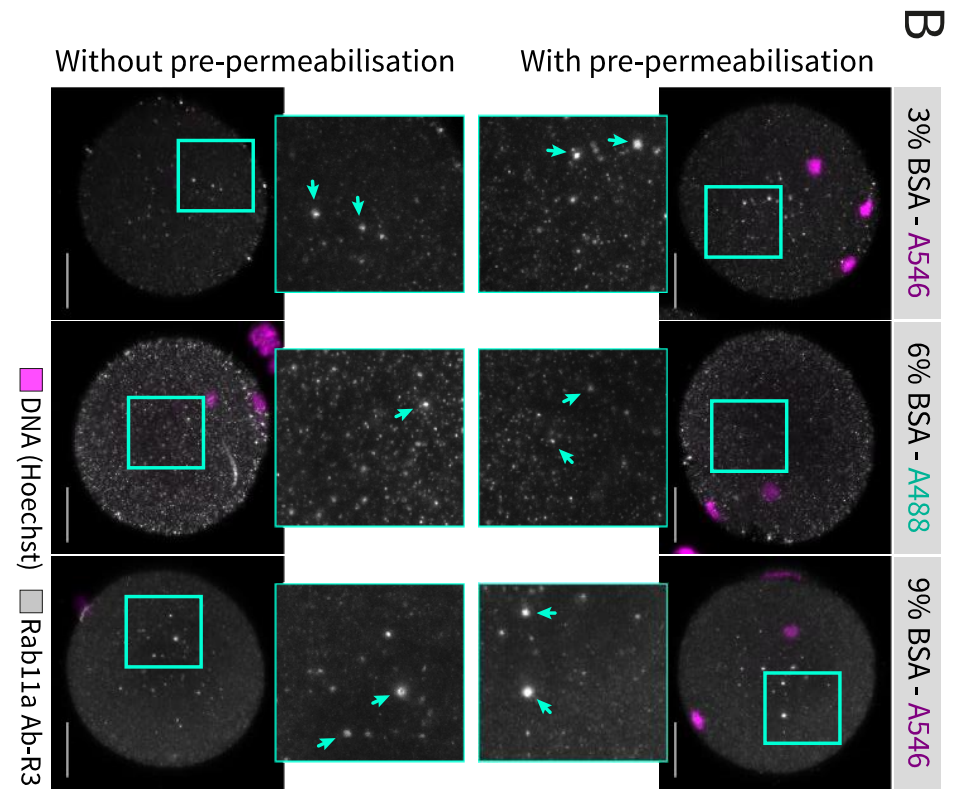

$\infty$
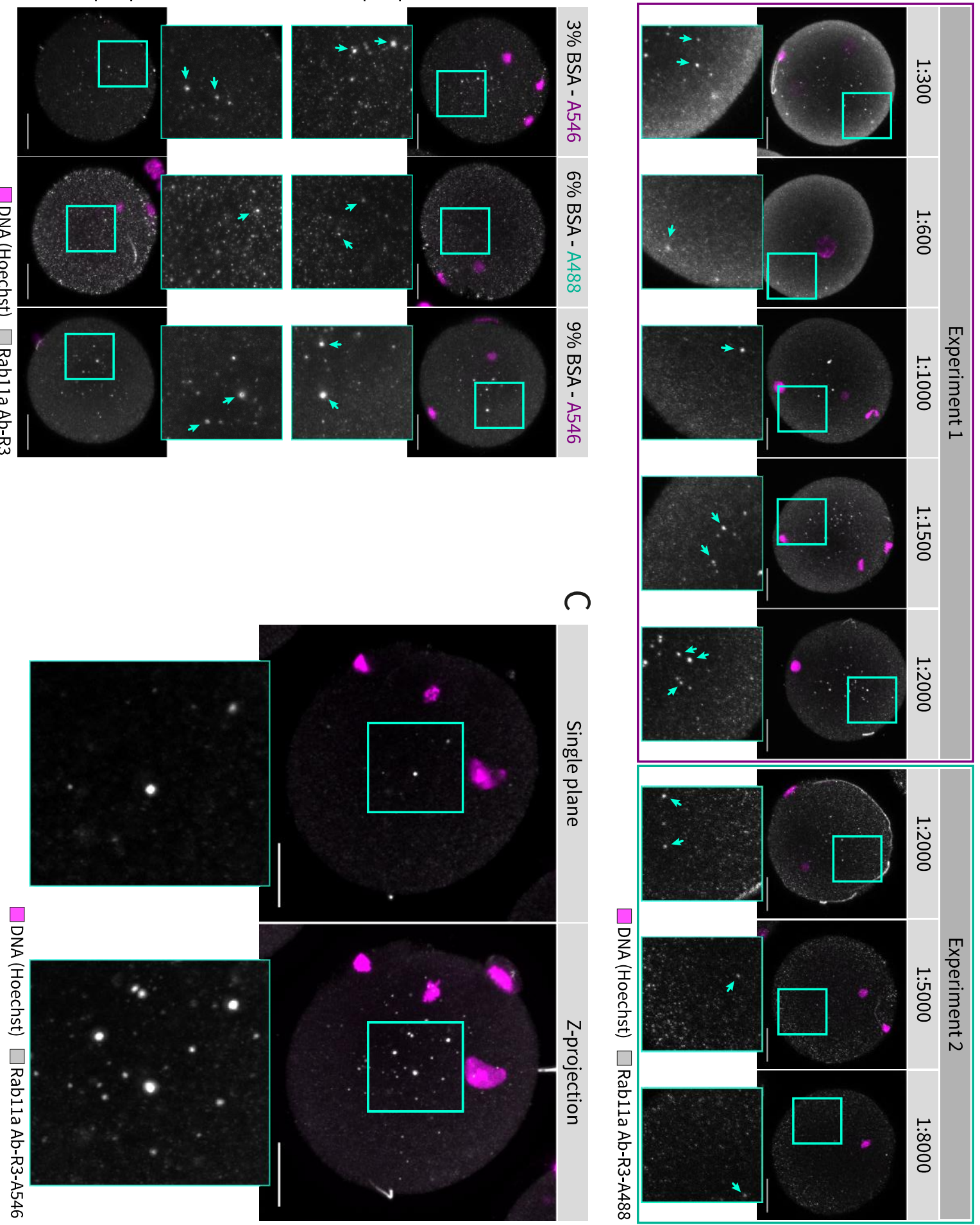

Figure 9: Optimisation of Rab11a immunofluorescence staining and imaging conditions. (A) Optimisation of Ab-R3 concentration in mouse zygotes. Zygotes were fixed 4 hpi (Experiment 1) and 3.5 hpi (Experiment 2), and subsequently stained using different dilutions of $A b-R 3$ (grey) and Hoechst (DNA; magenta) in two separate experiments. Cyan boxes indicate regions of interest amplified below; cyan arrows indicate Rab11a-positive vesicles inside the cytoplasm. Images are zprojections of $17 \times 1.5 \mu \mathrm{m}$ slices $(25.5 \mu \mathrm{m}$ total) around the centre of the zygote. Images taken with a Zeiss LSM-880 microscope. Scale bar $=20 \mu \mathrm{m}$. Representative images are shown, 2-3 zygotes were imaged per condition. (B) Optimisation of fixation, blocking and staining conditions. Zygotes were fixed 3.5 hpi and stained with Ab-R3 (grey) and Hoechst (DNA; magenta) with different conditions; they were either pre-permeabilised in $0.25 \%$ Triton-X or not, then blocked in either $3 \%$, $6 \%$ or $9 \%$ BSA in PBT, and the fluorophore used in the secondary antibody was either A546 (for 3\% and 9\% BSA) or A488 (for 
$6 \%$ BSA). Cyan boxes indicate regions of interest amplified below; cyan arrows indicate Rab11a-positive vesicles inside the cytoplasm. Images are z-projections of $17 \times 1.5 \mu \mathrm{m}$ slices (25.5 $\mathrm{mm}$ total) around the centre of the zygote. Images taken with a Zeiss LSM-800 microscope. Scale bar $=20 \mu \mathrm{m}$. Representative images are shown, 3-5 zygotes were imaged per condition. (C) Final conditions used for subsequent Rab11a immunofluorescence staining and imaging. Zygotes were pre-permeabilised and fixed 2 hpi, blocked in 12\% BSA, stained with Ab-R3 (grey) at a dilution of 1:2000 as well as Hoechst (DNA; magenta), and the A546 fluorophore secondary antibody was used. Cyan boxes indicate regions of interest amplified below. The single plane shows the central plane of the zygote; z-projections is of $17 \times 1.5 \mu \mathrm{m}$ slices (25.5 $\mu \mathrm{m}$ total) around the centre of the zygote. Images taken with a Zeiss LSM-880 microscope. Scale bar $=20 \mu \mathrm{m}$. Representative image is shown, a total of 8 zygotes were imaged in this group as part of an experimental series repeated 3 times for quantifications shown in Figure 11.

\section{Confirming co-localisation of Rab11a antibody staining and Rab11a-GFP in fixed zygotes}

Now that the conditions for Rab11a immunofluorescence were optimised, I wanted to confirm that the Rab11a-positive vesicles seen in live imaging by overexpression of mRNA corresponded to the vesicles observed in the immunofluorescence staining images. Furthermore, I wanted to see if the same accumulation that was seen in the cortical region adjacent to the male pronucleus when imaging mScarlet-Rab11a, as seen in Figure 7, could be observed by immunofluorescence staining. As the A561 laser was used to excite the A546 fluorophore of the secondary antibody, live zygotes expressing EGFP-Rab11a mRNA (as opposed to mScarlet-Rab11a) were observed under the confocal microscope after fertilisation, in individual oil-covered medium droplets on the imaging dish. As soon as the EGFPRab11a accumulation could be seen adjacent to the male pronucleus in a zygote, this cell was prepermeabilised and fixed immediately. The zygotes were then stained for Rab11a as optimised, and imaged. These results are seen in Figure 10, with two examples shown.

In Example 1, a z-projection of 17x1.5 $\mu \mathrm{m}$ around the centre of the zygote is shown. EGFP spots could still be observed in the cytoplasm, co-localising with the antibody signal, as indicated by the cyan arrows. This confirms that the "Rab11a-positive vesicles" are the same structures in live imaging using mRNA expression, and immunofluorescence using Ab-R3; however, it does not exclude possible side effects of overexpression of the mRNA.

In Example 2, a single plane of a zygote is shown in which the male pronucleus was forming at the cortex, and appeared to be in the stage in which mScarlet-Rab11a accumulation adjacent to the forming male pronucleus would have been observed in the region of interest (cyan box). No Rab11apositive signal was observed in this region, whether from the Ab-R3 antibody, or the fixed EGFP. However, as the EGFP was also maintained in the cytoplasm in this example, as seen in the region of interest marked by the magenta box and the cyan arrows, it is possible that this is a consequence of the fixation and permeabilisation, which may disrupt membrane structures, including a potential Rab11a accumulation in the cortex. The fixation methods were not optimised further to confirm this localised, cortical accumulation in fixed cells, and we instead later focused on further live imaging and functional approaches. 
Now that the antibody was confirmed to stain the same cytoplasmic vesicles as observed in live imaging, I wanted to confirm the trends observed in Figure 7, where vesicle number peaked around time of pronuclear formation, using immunofluorescence. 


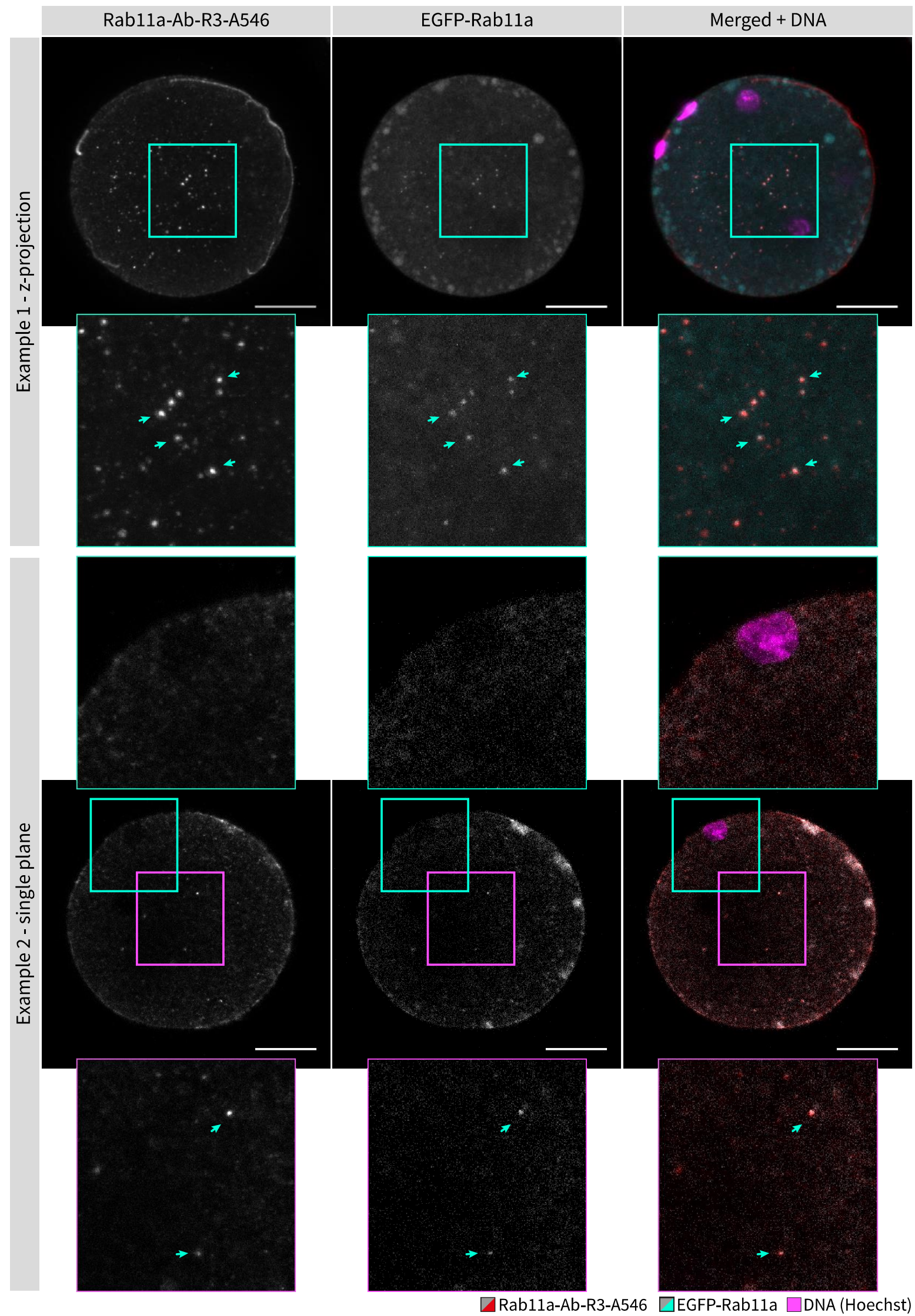

Figure 10: Co-localisation of EGFP-Rab11a and Rab11a immunofluorescence staining. Zygotes expressing EGFP-Rab11a mRNA were observed under a confocal microscope, and fixed at the time point of pronuclear formation, during which an accumulation of EGFP-Rab11a was observed adjacent to the male pronucleus. Fixed zygotes were stained with Ab-R3-A546 
(grey in left panels, red in right merged panels) and Hoechst (DNA; magenta in right merged panels), and these were imaged together with the fixed EGFP-Rab11a (grey in middle panels, cyan in right merged panels). Cyan and magenta boxes indicate regions of interest in the cytoplasm or at cortex zoomed-in above/below; cyan arrows indicate Rab11a-positive vesicles inside the cytoplasm. Example 1 shows a z-projection of $17 x 1.5 \mu \mathrm{m}$ slices $(25.5 \mu \mathrm{m}$ total) around the centre of the zygote; example 2 shows a single plane at which the forming male pronucleus is visible. Images taken with a Zeiss LSM-880 microscope. Scale $b a r=20 \mu \mathrm{m}$. Two representative examples of a total of seven zygotes from one experiment are shown.

\section{Immunofluorescence staining of Rab11a in zygotes at different time points after insemination}

In Figure 7, live imaging of mScarlet-Rab11a revealed that Rab11a-positive vesicles appeared at fertilisation, increased at pronuclear formation, and decreased again. I now wanted to confirm these results using the optimised Rab11a immunofluorescence staining. For this, zygotes were fixed at different time points after insemination and stained for Rab11a using Ab-R3. These results are seen in Figure 11. It is important to note that for the live imaging results seen in Figure 7, it was possible to set the time point of pronuclear formation for each zygote as time point 0 , and therefore see the dynamics of Rab11a-positive vesicles relative to pronuclear formation. However, for these fixation experiments, fixations were done relative to the time of insemination, not pronuclear formation. While no pronuclei had formed in the $2 \mathrm{hpi}$ and $3 \mathrm{hpi} \mathrm{groups,} \mathrm{pronuclei} \mathrm{began} \mathrm{forming} \mathrm{anywhere}$ between $3.5 \mathrm{hpi}$ and $5.5 \mathrm{hpi}$, resulting in heterogenous populations of zygotes in the $4 \mathrm{hpi}$ and $5 \mathrm{hpi}$ groups; after $6 \mathrm{hpi}$, all zygotes had formed pronuclei.

In Figure 11A, representative images from three experiments are shown. At $2 \mathrm{hpi}$, the zygote was freshly fertilised, and no polar body had formed yet. Rab11a-positive vesicles were already clearly visible in the cytoplasm, as seen in the magnified region of interest. At $3 \mathrm{hpi}$, the polar body extrusion had usually occurred, and a similar number of vesicles was seen in the cytoplasm as at $2 \mathrm{hpi}$. At $4 \mathrm{hpi}$, when some pronuclei began to form, the cytoplasm was filled with many Rab11a-positive vesicles, more than observed at $2 \mathrm{hpi}$ and $3 \mathrm{hpi}$. At $5 \mathrm{hpi}$, the zygotes showed very heterogenous results, and therefore two representative examples are shown. In the first, the pronuclei were only just forming, and many vesicles were seen in the cytoplasm. In the second, the pronuclei had already formed, and only very faint spots were seen in the cytoplasm. After this time point, no more or only very few clear vesicles could be detected in the cytoplasm. This qualitative trend - that vesicles increase up to pronuclear formation, then decrease in number to near zero - was the same as observed in live imaging of mScarlet-Rab11a (Figure 7).

Next, these results were quantified. At first, a manual counting approach of the bottom half of the zygote was used, as was done for the live imaging; the results are seen in Figure 11B. Indeed, this quantification confirmed the same trend with similar values as counted in the live imaging shown in Figure 7. A One-Way ANOVA test was done to identify statistical significance of this dataset, which revealed a significant difference between groups overall $(p<0.001)$; Tukey's multiple comparison test 
allowed further comparison between the different time points. Before pronuclear formation, a mean number of $28.24 \pm 10.62$ vesicles were counted at $2 \mathrm{hpi}$, and $35.53 \pm 23.59$ vesicles were counted at $3 \mathrm{hpi}$, which did not show significant difference ( $p>0.05$, One-way Tukey's ANOVA). From 3 hpi to $4 \mathrm{hpi}$, there was a significant increase to a mean of $99.38 \pm 50.68$ vesicles $(p<0.001$, One-way Tukey's ANOVA). The decrease from $4 \mathrm{hpi}$ to $5 \mathrm{hpi}$ to $57.09 \pm 62.62$ was also statistically significant $(p<0.001$, One-way Tukey's ANOVA), and the high standard deviation at 5 hpi reflects the fact that the zygotes at this time point were in different stages relative to pronuclear formation. The further drop from $5 \mathrm{hpi}$ to $6 \mathrm{hpi}$ to $18.13 \pm 27.05$ vesicles manually counted per half zygote was statistically significant $(p<0.01$, One-way Tukey's ANOVA). After $6 \mathrm{hpi}$, no statistically significant change was observed between subsequent time points anymore; only very few vesicles could be observed in these time points, if any. These results are very similar to those seen in the live imaging experiments, as Rab11apositive vesicles increased from fertilisation to pronuclear formation, and decreased again afterwards to near zero. However, there were several issues with the manual counting approach, as a possible bias was difficult to exclude; the samples could not be counted 'blindly' as the stage of pronuclear formation and migration was always obvious to the counter. Also, while no more large, bright spots were seen in most zygotes after pronuclear formation, some faint spots remained, and it was unclear how to classify these in the manual count. For this reason, I optimised automatic spot detection using IMARIS Software.

The exact settings used for the IMARIS Spot detection can be found in Methods Section 2.3. In short, a section of $25 \times 25 \times 16.5 \mu \mathrm{m}(x, y, z$ respectively) around the centre of the zygote was analysed in IMARIS. All spots were first detected, regardless of volume. As the software detected background noise as spots that all had the same volume of $0.0283 \mu \mathrm{m}^{3}$, these minimum-volume spots were filtered out. What was left were spots ranging from $0.037 \mu \mathrm{m}^{3}$ to the largest spot detected at $26.13 \mu \mathrm{m}^{3}$. The total number of spots detected for each time point of fixation are shown in Figure 11C. Over 300 spots were detected at each time point, both before, during, and after pronuclear formation, even when no Rab11a-positive vesicles could be seen with the human eye. It is likely that this was due to the spotlike background signal of the antibody, and therefore, further sorting was necessary.

These spots were next binned according to volume, as can be seen in Figure 11D. The largest bin pool, $>2 \mu \mathrm{m}^{3}$, was chosen based on the largest and brightest Rab11a-positive vesicles, very obvious to the human eye. The next bin pool, $>1 \mu \mathrm{m}^{3}$ to $\leq 2 \mu \mathrm{m}^{3}$, was chosen as the group still visible to the human eye, but not as clearly, and thus may have on occasion been missed in the manual count. The smallest bin pool, $>0.5 \mu \mathrm{m}^{3}$ to $\leq 1 \mu \mathrm{m}^{3}$, included the group of vesicles that were difficult to classify in the manual count as they were only marginally brighter or larger than background spots. As seen in the resulting bar chart, the trend observed in all of these binned groups is very similar to what was seen in the 
manual count of same fixed cells shown in Figure $11 \mathrm{~B}$ as well as the live imaging manual count in Figure 7, though due to a smaller volume of the zygote used for this quantification, the exact values are lower. None of the groups show a significant difference between the $2 \mathrm{hpi}$ and $3 \mathrm{hpi}$ groups ( $p>0.05$, One-way Tukey's ANOVA). Between $3 \mathrm{hpi}$ and $4 \mathrm{hpi}$, all groups show a statistically significant increase; the largest group of spots, $>2 \mu \mathrm{m}^{3}$, increase from a mean of $5.95 \pm 4.62$ spots at $3 \mathrm{hpi}$ to $15.58 \pm 8.11$ spots at $4 \mathrm{hpi}\left(\mathrm{p}<0.001\right.$, One-way Tukey's ANOVA); the middle group of spots, $>1 \mu \mathrm{m}^{3}$ to $\leq 2 \mu \mathrm{m}^{3}$, sees an increase in the mean number of spots from $6.68 \pm 4.11$ spots to $13.50 \pm 5.03$ spots ( $p<0.01$, One-way Tukey's ANOVA); the group of spots ranging in volume from $>0.5 \mu \mathrm{m}^{3}$ to $\leq 1 \mu \mathrm{m}^{3}$ also show a statistically significant increase from $13.32 \pm 8.39$ spots at $3 \mathrm{hpi}$ to $21.38 \pm 9.38$ spots at $4 \mathrm{hpi}$ $(p<0.05$, One-way Tukey's ANOVA). While the smaller two groups do not show a significant difference between the $4 \mathrm{hpi}$ and $5 \mathrm{hpi}$ groups, there is a difference seen in the group of spots above a volume of $2 \mu \mathrm{m}^{3}$, which decreases to $8.32 \pm 11.29$ spots ( $\mathrm{p}<0.01$, One-way Tukey's ANOVA). Between $5 \mathrm{hpi}$ and $6 \mathrm{hpi}$, all groups show a significant drop in number of spots detected; the largest group of spots, $>2 \mu \mathrm{m}^{3}$, decrease to a mean of $1.86 \pm 4.26$ spots at $6 \mathrm{hpi}(\mathrm{p}<0.01$, One-way Tukey's ANOVA); the middle group of spots, $>1 \mu \mathrm{m}^{3}$ to $\leq 2 \mu \mathrm{m}^{3}$, sees a decrease in the mean number of spots from $12.14 \pm 12.84$ spots at $5 \mathrm{hpi}$ to $5.48 \pm 4.63$ spots at $6 \mathrm{hpi}(\mathrm{p}<0.01$, One-way Tukey's ANOVA); the group of spots from $>0.5 \mu \mathrm{m}^{3}$ to $\leq 1 \mu \mathrm{m}^{3}$ show a significant drop from $27.14 \pm 12.83$ spots at $5 \mathrm{hpi}$ to $15.90 \pm 9.01$ spots at $6 \mathrm{hpi}(\mathrm{p}<0.001$, One-way Tukey's ANOVA). This smallest group of spots is the only one to show a further significant drop between $6 \mathrm{hpi}$ and $8 \mathrm{hpi}$, down to $7.43 \pm 8.05$ spots detected ( $p<0.05$, One-way Tukey's ANOVA); between $8 \mathrm{hpi}$ and $10 \mathrm{hpi}$, none of the binned groups of spots show any statistically significant difference.

Overall, the results in all of these volume groups resemble the trend from the manual count of both fixed and live cells shown previously. It is, of course, possible that this analysis method and binning still excluded smaller spots that were true Rab11a-positive vesicles, or even included some spots that were not true vesicles. Therefore, we cannot exclude the possible existence of smaller Rab11a-positive vesicles, nor assume that all spots detected, especially those in the smallest volume group, reflect true Rab11a-positive vesicles. Therefore, we cannot be certain if the number of vesicles counted in the region of interest is exact. However, as this automatic counting confirms the same trend already seen with the human eye, it is likely that the overall trend is true.

Next, I wanted to use this optimised staining and imaging to confirm the difference in vesicle number between MII oocytes and fertilised zygotes, as well as to investigate how the two different Rab11a perturbation methods used previously - brefeldin A and the dominant-negative Rab11a $a^{525 N}$ - affected the endogenous levels of these Rab11a-positive vesicles in zygotes. 

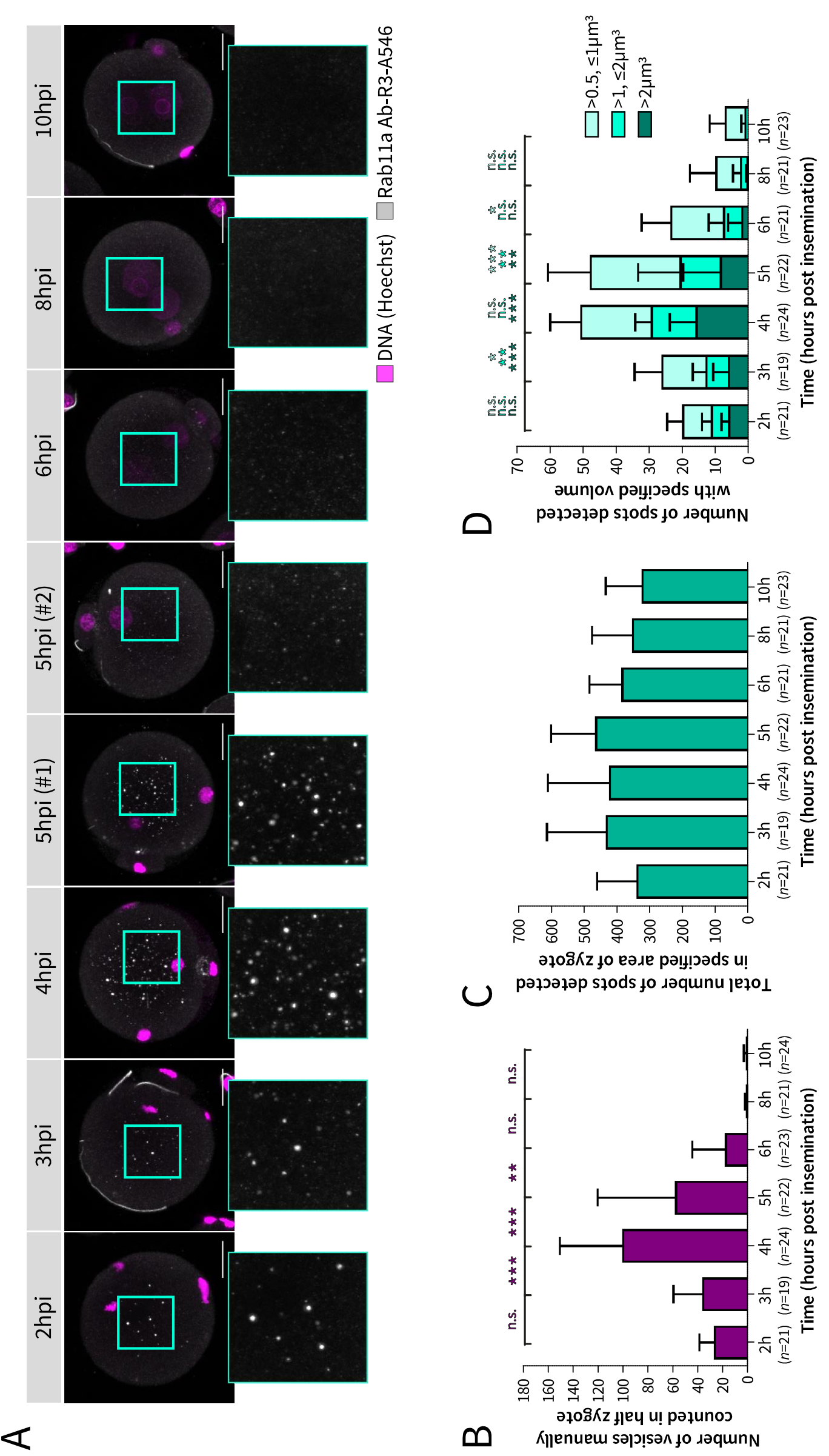
Figure 11: Immunofluorescence staining and quantification of Rab11a-positive vesicles in zygotes at different time points after insemination. (A) Confocal images of zygotes fixed at different time points, stained with Rab11a-Ab-R3 (grey) and Hoechst (DNA; magenta); cyan boxes indicate region of interest zoomed in next to each zygote. z-projection of $17 \times 1.5 \mu \mathrm{m}$ slices. Images taken with a Zeiss LSM-880 microscope. Scale bar $=20 \mu \mathrm{m}$. Representative images from a total of 3 experiments are shown; n-numbers for each time point are shown under the respective bar. (B) Number of vesicles manually counted in half zygote over time. The bottom half of each zygote was used to manually count vesicles. Black error bar = one standard deviation. Statistical significance between time points calculated using One-way ANOVA with Tukey's multiple comparison test; * $p \leq 0.05,{ }^{* *} p \leq 0.01,{ }^{* * *} p \leq 0.001$. (C) Total number of spots above a volume of $0.0283 \mu \mathrm{m}^{3}$ detected in a $25 \times 25 \times 16.5 \mu \mathrm{m}^{3}$ central volume of each zygote, automatically detected using IMARIS Spot detection function. Black error bar = one standard deviation. (D) Results from (C) were binned according to volume of detected spots into three categories; $>2 \mu^{3}{ }^{3}$ (dark cyan), $>1 \mu \mathrm{m}^{3}$ to $\leq 2 \mu \mathrm{m}^{3}$ (medium cyan) and $>0.5 \mu \mathrm{m}^{3}$ to $\leq 1 \mu \mathrm{m}^{3}$ (light cyan). Black error bar = one standard deviation. Statistical significance between time points calculated using One-way ANOVA with Tukey's multiple comparison test; * $p \leq 0.05, * *$ $p \leq 0.01, * * * 0 \leq 0.001$.

\section{Rab11a-positive vesicles are absent in MII oocytes and zygotes with perturbed Rab11a function}

Now that Rab1la antibody staining and subsequent automatic spot quantification had been optimised, I wanted to confirm an observation made but not quantified with live imaging of mScarletRab11a in Figure 7; that MII oocytes contained no (or only few) Rab11a-positive vesicles, compared to vesicle-containing early zygotes. The results of this are shown in Figure 12A and B. Fixed MII oocytes along with zygotes fixed 3 hpi were stained with Rab11a-Ab-R3 (grey) and Hoechst (DNA; magenta). After imaging, spots were automatically detected and binned as for Figure 11; representative images from 3 experiments are seen in Figure 12A, and the quantification in Figure 12B. As is evident in both the images shown and from the quantification, MIl oocytes had significantly fewer spots in all specified volume groups than zygotes $3 \mathrm{hpi}$. For the largest spot volume group of $>2 \mu \mathrm{m}^{3}$, no vesicles were detected in any MII oocytes, while a mean of $6.26 \pm 4.94$ vesicles were detected in the zygotes ( $p<0.001$, Welch's t-test). In the medium spot volume group of $>1 \mu \mathrm{m}^{3}$ to $\leq 2 \mu \mathrm{m}^{3}$, again zero vesicles were detected in the MII oocytes, while $6.70 \pm 4.12$ spots of this volume were detected in the zygote ( $p<0.001$, Welch's t-test). In the smallest volume group of $>0.5 \mu \mathrm{m}^{3}$ to $\leq 1 \mu \mathrm{m}^{3}, 3.07 \pm 2.72$ spots were detected in the MII oocyte, and $14.22 \pm 7.37$ were detected in the zygote $(p<0.001$, Welch's t-test). This confirms the side observation made in live imaging of mScarlet-Rab11a that the large Rab11apositive vesicles seen in early zygotes are not present prior to fertilisation.

In this project, two functional approaches to disrupting Rab11a function were used; first of all, brefeldin A was used previously by Kathleen Scheffler, and was also used for my part of the project later on. Secondly, expression of a dominant-negative Rab11a variant, $\mathrm{S} 25 \mathrm{~N}$, was used as an alternate way to disrupt Rab11a function. Both of these methods have been previously described to disrupt Rab11a localisation in oocytes expressing fluorescently tagged Rab11a imaged live (Holubcová et al., 2013), but this had not been confirmed in zygotes, and not looking at endogenous Rab11a levels using immunofluorescence as opposed to overexpression of mRNA. Therefore, the effect of brefeldin A

(Figure $12 \mathrm{C}$ and $\mathrm{D}$ ) and expression of SNAP-Rab11a ${ }^{S 25 N}$ mRNA (Figure 12E and F) on Rab11a-positive 
vesicles detected in immunofluorescence was tested. For this, zygotes incubated in methanol or brefeldin A, or expressing wild-type or dominant-negative SNAP-Rab11a mRNA, were fixed $3 \mathrm{hpi}$, then stained, imaged and analysed as established.

In Figure 12C, representative images from 3 experiments treating zygotes with methanol or brefeldin A are shown. While Rab11a-positive vesicles were present in the cytoplasm of the methanol-treated zygotes, no such bright spots were observed in the brefeldin A-treated zygotes. The quantification shown in Figure 12D confirms this; all three groups of spots with different volumes were decreased significantly in the brefeldin A group. For the largest spot volume group of $>2 \mu \mathrm{m}^{3}$, a mean of $6.26 \pm 4.94$ spots were detected in the methanol-treated zygotes, compared to $0.04 \pm 0.21$ spots in brefeldin A-treated zygotes ( $p<0.001$, Welch's t-test). In the medium spot volume group of $>1 \mu^{3}$ to $\leq 2 \mu \mathrm{m}^{3}, 6.70 \pm 4.12$ spots of this volume were detected in the methanol-treated zygotes, compared to $0.30 \pm 0.63$ spots in the brefeldin A-treated zygotes ( $p<0.001$, Welch's t-test). In the smallest volume group of $>0.5 \mu \mathrm{m}^{3}$ to $\leq 1 \mu \mathrm{m}^{3}$, a mean of $14.22 \pm 7.37$ spots were detected in the methanol-treated zygotes compared to $6.09 \pm 5.42$ in the brefeldin A-treated zygotes ( $p<0.001$, Welch's t-test).

In Figure 12E, representative images from 3 experiments with zygotes expressing either wild-type SNAP-Rab11a ${ }^{W T}$ or dominant-negative SNAP-Rab11 ${ }^{S 25 N}$ mRNA are shown. As in methanol-treated zygotes, Rab11a-positive vesicles were visible in the cytoplasm of the zygotes expressing SNAP$R a b 11 a^{W T}$, but no such bright spots were seen in the SNAP-Rab11a $a^{S 25 N}$ expressing zygotes. The quantification shown in Figure $12 \mathrm{~F}$ shows that all three groups of spots with different volumes were decreased significantly in the dominant-negative group. For the largest spot volume group of $>2 \mu^{3}$, a mean of $9.65 \pm 6.44$ spots were detected in the zygotes expressing wild-type Rab11a, compared to $0.05 \pm 0.21$ spots in zygotes expressing dominant-negative Rab11a ( $p<0.001$, Welch's t-test). In the medium spot volume group of $>1 \mu \mathrm{m}^{3}$ to $\leq 2 \mu \mathrm{m}^{3}, 7.13 \pm 4.22$ spots of this volume were detected in the SNAP-Rab11aWT expressing zygotes, compared to $0.27 \pm 0.77$ spots in the SNAP-Rab11 ${ }^{\text {S25N }}$ expressing zygotes ( $p<0.001$, Welch's t-test). In the smallest volume group of $>0.5 \mu \mathrm{m}^{3}$ to $\leq 1 \mu \mathrm{m}^{3}$, a mean of $12.17 \pm 7.30$ spots were detected in the wild-type Rab11a zygotes compared to $1.05 \pm 2.59$ in the dominant-negative group ( $p<0.001$, Welch's t-test).

These results indicate that treatment with brefeldin A and expression of SNAP-Rab11a ${ }^{S 25 N}$ mRNA, which both disrupt the early fast migration of the male pronucleus, disrupt the same Rab11a-positive vesicles that we detect in our early zygotes, but were not detected in later zygotes or in MII oocytes.

Finally, all of these observations support the statement made previously that the spots detected with a volume of $>0.5 \mu \mathrm{m}^{3}$ to $\leq 1 \mu \mathrm{m}^{3}$ were likely a mixture of true Rab11a-positive vesicles and some nonspecific background spots. There was a statistically significant difference between the perturbed and 
control cells as well as MII oocytes and zygotes, indicating that a large portion of spots detected in this group were biologically relevant in this process. However, while in MII oocytes, no spots of the larger volumes were found, the number of these small spots detected was not zero, making it possible that some background spots are being detected with this size. However, it still cannot be excluded that these spots are smaller Rab11a-positive vesicles which are indeed present in MII oocytes and are resistant to brefeldin A and Rab11a ${ }^{525 \mathrm{~N}}$ perturbation.

After these different immunofluorescence experiments, the next focus of the project was to continue optimising live imaging of Rab11a and other structures in higher temporospatial resolution, to further investigate its possible role in early pronuclear migration. 
A

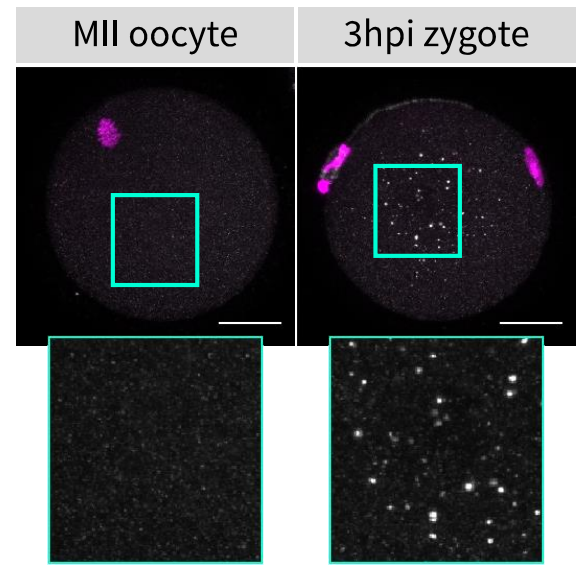

$\square$ DNA (Hoechst) $\square$ Rab11a Ab-R3-A546

C

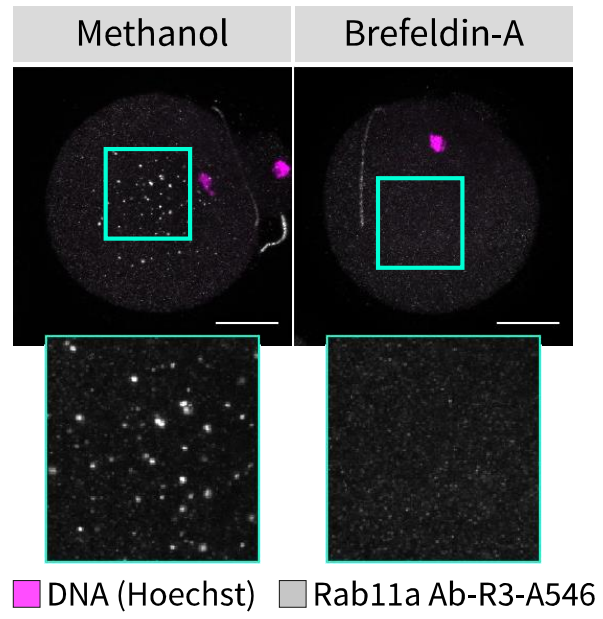

$\mathrm{E}$

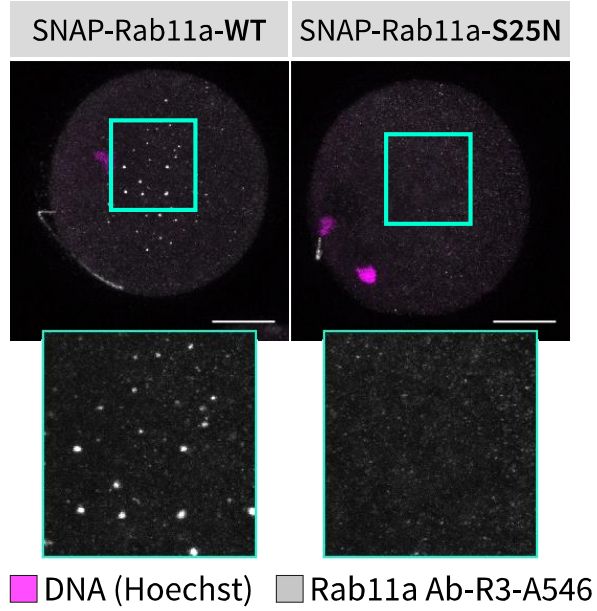

B

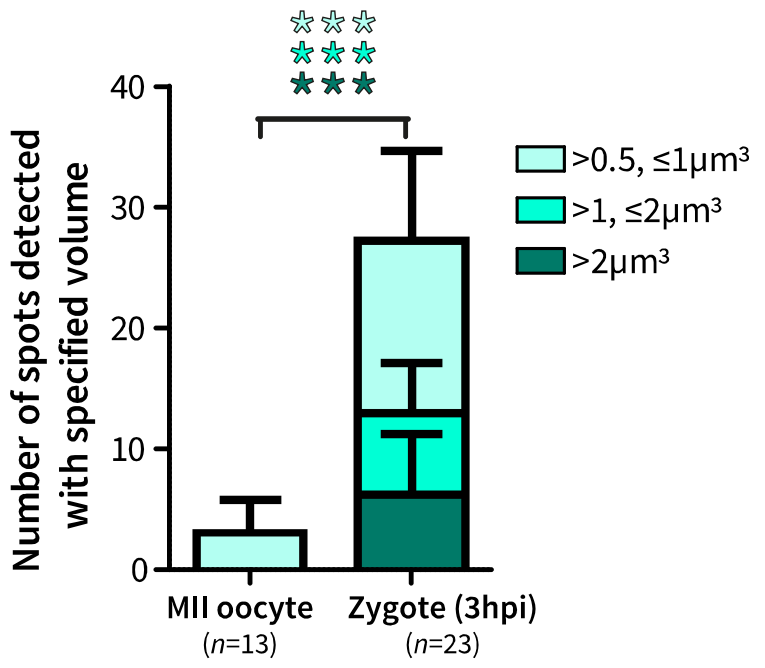

D

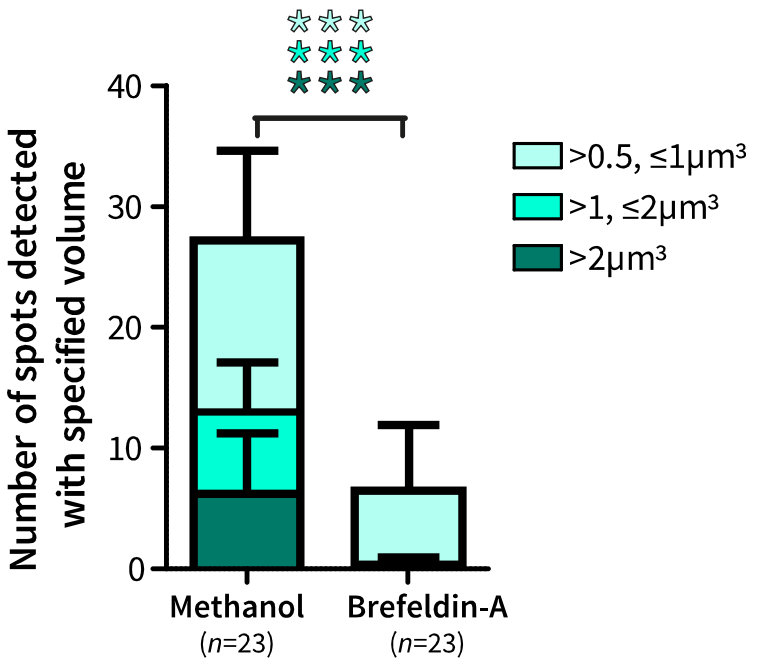

F

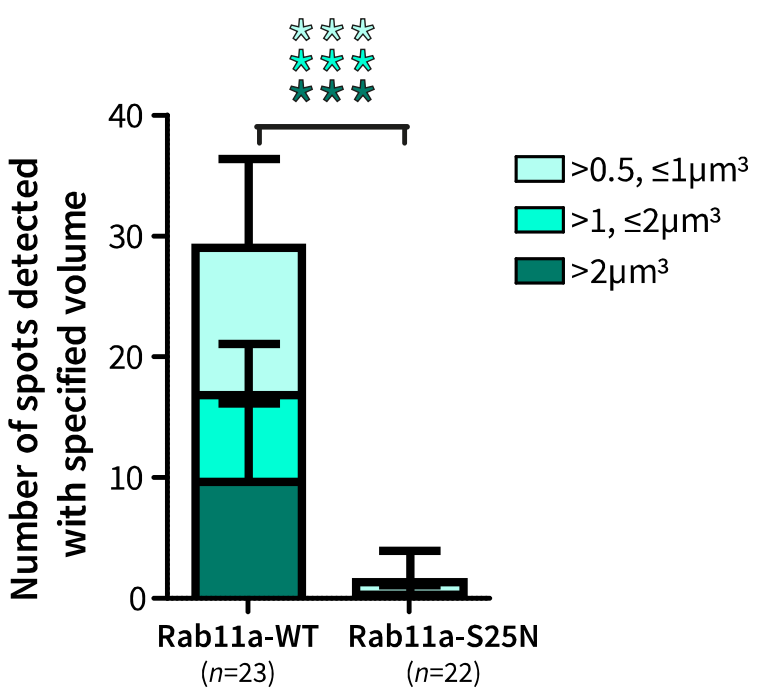

Figure 12: Immunofluorescence staining of MII oocytes, brefeldin A-treated and SNAP-Rab11a ${ }^{S 25 N}$-expressing mouse zygotes. (A) Confocal images of fixed MIl oocytes and zygotes 3 hpi stained with Rab11a-Ab-R3 (grey) and Hoechst (DNA; magenta); cyan boxes indicate region of interest zoomed in below each zygote. z-projection of $17 \times 1.5 \mu \mathrm{m}$ slices. Images taken with a Zeiss LSM-800 microscope. Scale bar $=20 \mu \mathrm{m}$. Representative images from a total of 3 experiments are shown; $\mathrm{n}$ numbers for each condition are shown under the respective bar. (B) Vesicles in MIl oocytes and zygotes 3 hpi were automatically detected using IMARIS spot detection function and were binned according to volume of detected spots into three categories; $>2 \mu \mathrm{m}^{3}$ (dark cyan), $>1 \mu \mathrm{m}^{3}$ to $\leq 2 \mu \mathrm{m}^{3}$ (medium cyan) and $>0.5 \mu \mathrm{m}^{3}$ to $\leq 1 \mu \mathrm{m}^{3}$ (light cyan). Black error bar = one standard deviation. Statistical significance between conditions calculated with Unpaired, two-tailed t-test with Welch's 
correction; * $p \leq 0.05, * * p \leq 0.01, * * * p \leq 0.001$. (C) Confocal images of fixed zygotes 3 hpi treated with either methanol or brefeldin A, stained with Rab11a-Ab-R3 (grey) and Hoechst (DNA; magenta); cyan boxes indicate region of interest zoomed in below each zygote. z-projection of $17 \times 1.5 \mu \mathrm{m}$ slices. Images taken with a Zeiss LSM-800 microscope. Scale bar $=20 \mu \mathrm{m}$. Representative images from a total of 3 experiments are shown; $\mathrm{n}$-numbers for each condition are shown under the respective bar. (D) Vesicles in zygotes treated with methanol and brefeldin A were automatically detected using IMARIS spot detection function and were binned according to volume of detected spots into three categories; $>2 \mu m^{3}$ (dark cyan), $>1 \mu m^{3}$ to $\leq 2 \mu m^{3}$ (medium cyan) and $>0.5 \mu \mathrm{m}^{3}$ to $\leq 1 \mu \mathrm{m}^{3}$ (light cyan). Black error bar = one standard deviation. Statistical significance between conditions calculated with Unpaired, two-tailed t-test with Welch's correction; * $p \leq 0.05, * * p \leq 0.01, * * * p \leq 0.001$. (E) Confocal images of fixed zygotes 3 hpi expressing either SNAP-Rab11aWT or SNAP-Rab11a ${ }^{\mathrm{S} 25 \mathrm{~N}}$ mRNA, stained with Rab11a-Ab-R3 (grey) and Hoechst (DNA; magenta); cyan boxes indicate region of interest zoomed in below each zygote. z-projection of $17 \times 1.5 \mu \mathrm{m}$ slices. Images taken with a Zeiss LSM-800 microscope. Scale bar $=20 \mu \mathrm{m}$. Representative images from a total of 3 experiments are shown; $\mathrm{n}$-numbers for each condition are shown under the respective bar. (F) Vesicles in zygotes expressing wild-type or dominant-negative Rab11a were automatically detected using IMARIS spot detection function and were binned according to volume of detected spots into three categories; $>2 \mu \mathrm{m}^{3}$ (dark cyan), $>1 \mu \mathrm{m}^{3}$ to $\leq 2 \mu \mathrm{m}^{3}$ (medium cyan) and $>0.5 \mu \mathrm{m}^{3}$ to $\leq 1 \mu \mathrm{m}^{3}$ (light cyan). Black error bar = one standard deviation. Statistical significance between conditions calculated with Unpaired, two-tailed t-test with Welch's correction; * $p \leq 0.05,{ }^{* *} p \leq 0.01,{ }^{* * *} p \leq 0.001$. 


\subsection{Rab11a and actin nucleators accumulate adjacent to the forming male pronucleus}

\section{Live imaging of mScarlet-Rab11a in high temporospatial resolution at the forming pronuclei}

In Figure 7, three observations were made: Firstly, that MII oocytes did not contain large Rab11apositive vesicles as observed in zygotes; secondly, that these Rab11a-positive vesicles were present from fertilisation until shortly after pronuclear formation, when they peaked; and thirdly, that in some zygotes, an accumulation of mScarlet-Rab11a was seen at the cortical region adjacent to the forming male pronucleus. The former two observations were able to be confirmed by immunofluorescence in the last sections, while the latter was not confirmed in zygotes fixed at the time point of pronuclear formation (shown in Figure 10). This could have been for two reasons; either, this accumulation was real and possibly functional, but simply was not maintained under the fixation and permeabilisation protocol we used; or, it was a possible artefact of mRNA overexpression. Therefore, the rest of this thesis focused on finding alternative ways to investigate this accumulation, with a variety of observational and functional techniques.

First of all, I wanted to better characterise the mScarlet-Rab11a accumulation seen when imaging mScarlet-Rab11a by imaging it with a higher temporospatial resolution. In Figure 7, whole zygotes were imaged with a z-resolution of $3 \mu \mathrm{m}$ and time resolution of 15 minutes. Now, I wanted to optimise imaging settings to visualise the area surrounding the forming male pronucleus, in particular the cortical region directly overlaying it, and also compare this to the area surrounding the forming female pronucleus. For this, regions of $46.98 \times 46.98 \mu \mathrm{m}$, zoomed into the pronuclei of zygotes expressing mScarlet-Rab11a mRNA, were imaged every 3 minutes with a z-resolution of $1.5 \mu \mathrm{m}$. A few difficulties were encountered during this optimisation. First of all, the best way to see this cortical region and enrichment was when the sperm entry site and thus male DNA was located exactly in the central plane of the zygote at the cortex, as opposed to at the top or bottom of the zygote. Even when it was only slightly above the central plane, and therefore further away from the objective, the signal intensity of the mScarlet-Rab11a was weakened enough to make observation of the accumulation difficult. Another issue was that the zygotes were often rotating before pronuclear formation, especially as some zygotes had still-swimming sperm stuck in between the zona pellucida and the plasma membrane. This meant that in some cases, imaging had to be stopped, the zygote repositioned, and imaging continued again. The timing of pronuclear formation varied greatly between zygotes, meaning that when multiple zygotes were imaged, and some had already formed while others were still rotating, not all zygotes imaged would result in useful images. Lastly, while most male DNA after sperm entry would be located directly adjacent to the cortex, other sperm entered deeper into the oocyte, meaning it would not be lying right at the cortex. As Kathleen Scheffler showed in her initial experiments shown in the Introduction section 1.4, the male pronuclei that formed deeper inside the 
cell would often not show the initial fast migration that male pronuclei showed when they formed closer to the cortex. While these zygotes were initially excluded from imaging, later on, they were imaged to see how this would affect mScarlet-Rab11a accumulation. Thus, cortical male pronuclei as well as female pronuclei were imaged in 4 separate experiments, while male pronuclei forming deeper were imaged in only one experiment. These results are shown in Figure $\mathbf{1 3 .}$

In Figure 13A, the area of a zygote expressing mScarlet-Rab11a surrounding the male pronucleus is shown. Both the single z-plane of the centre of the male DNA, as well as a z-projection of $15 \times 1.5 \mu \mathrm{m}$ around this centre is shown. This male pronucleus formed close to the cortex, and a fertilisation cone was visible prior to pronuclear formation at time points $-0: 06$ and 0:00. In the third panel, the pronucleus had begun forming, and therefore, the last time point before pronuclear formation was

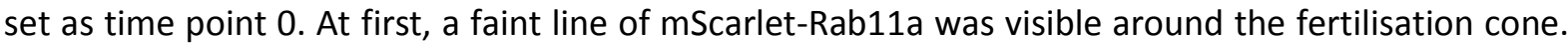
As the pronucleus forms, a bright accumulation was seen at 0:12, which was no longer visible after the pronucleus had migrated quickly away from the cortex at 0:21. Another observation that could be made from these higher time-resolution images was that the cytoplasmic Rab11a-positive vesicles appeared to be highly dynamic before and around pronuclear formation - there were some vesicles visible at $-0: 06$, fewer at 0:00, and many more at 0:03. At 0:12, when the accumulation by the cortex appeared, the cytoplasmic vesicles were not visible, but they were visible again at 0:21. These oscillations were not the focus of these experiments, and the imaging conditions did not allow quantification. However, the general observation that vesicle number oscillated with a period of approximately 3-6 time points (9-18 minutes), was made. As had already been shown in Figure 7, Rab11a-positive vesicles were no longer visible within 30 minutes after pronuclear formation, though these time points are not shown here.

In Figure 13B, the area of a zygote expressing mScarlet-Rab11a surrounding the female pronucleus and the polar body (PB) is shown. In the cytoplasm, highly dynamic vesicles were observed, which fluctuated in number and intensity over time. Also, some accumulation was apparent at the site of polar body extrusion and cytokinesis, but no obvious accumulation was seen by the female pronucleus as it formed and began its slower migration towards the centre.

In Figure 13C, the area of a zygote expressing mScarlet-Rab11a surrounding a male pronucleus that formed deeper inside the zygote is shown. Again, vesicles were seen in the cytoplasm, though fewer than in the other two examples shown. As the pronucleus formed with some distance to the cortex, no obvious mScarlet-Rab11a accumulation could be seen. This is a representative example of only four of these types of 'deep' male pronuclei with varying distances from the cortex. One of these, which formed a little closer to the cortex, showed some mScarlet-Rab11a accumulation. 
The quantification of these results is shown in Figure 13D. For each imaged zygote, a judgement was made by eye whether or not accumulation could be seen. As explained above, not all male pronuclei formed at the perfect angle to the objective to make this accumulation appear as bright as in the example shown in Figure 13A. However, bias was avoided as much as possible by repeating this quantification three times for each zygote, so that "yes" or "no" could be decided for each sample. In the case of male pronuclei forming close to the cortex, mScarlet-Rab11a enrichment could be seen in $30 / 37$ (81.08\%) of cases. No female pronuclei $(0 / 18 ; p<0.001$, two-tailed Fisher's exact test compared to male pronuclei), which all formed deeper inside the cell, showed such an accumulation. Of the male pronuclei that formed deeper inside the cell, $1 / 4$ (25\%; $p=0.0388$, two-tailed Fisher's exact test compared to male pronuclei) showed mScarlet-Rab11a enrichment at the cortical region adjacent to it.

In these results, we saw that higher temporospatial imaging revealed mScarlet-Rab11a enrichment adjacent to the majority of forming male pronuclei, but not female pronuclei, and not in most male pronuclei that form further from the cortex, possibly depending on distance from the cortex at pronuclear formation. They also showed that this imaging and its analysis were not always straightforward, but the observations made were nonetheless interesting and required further investigation. 


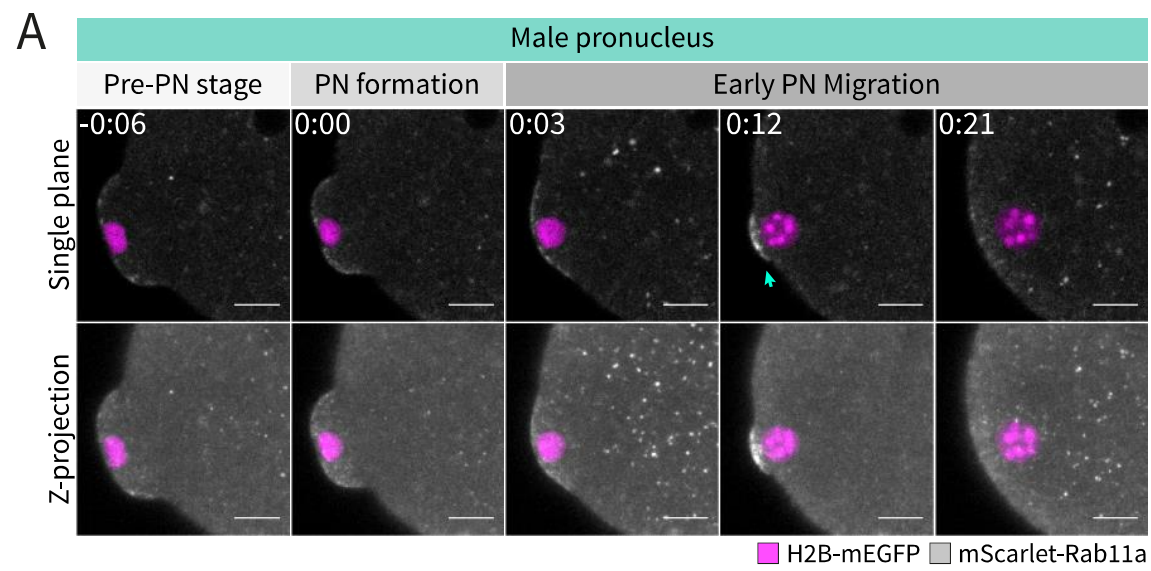

B

Female pronucleus

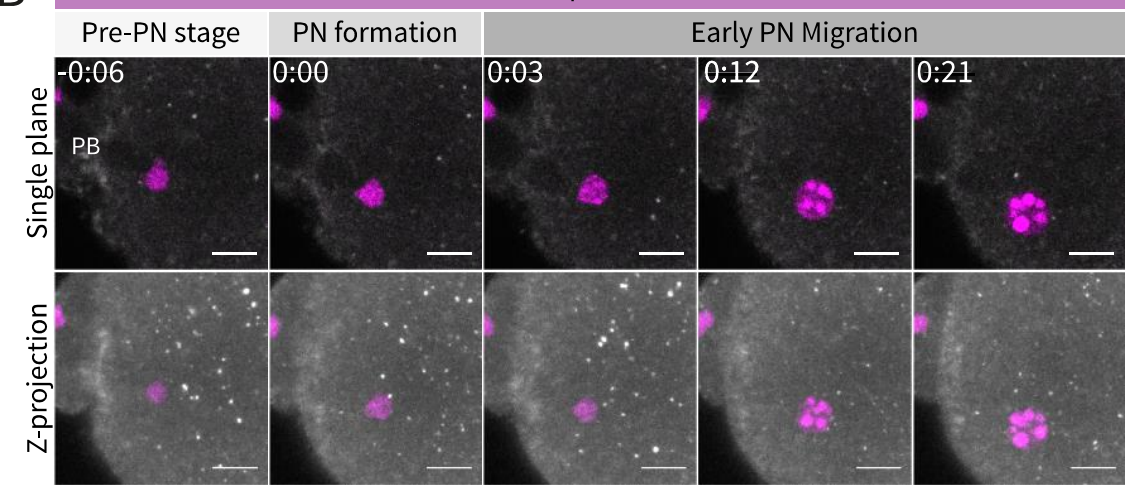

$\square$ H2B-mEGFP $\square$ mScarlet-Rab11a

C

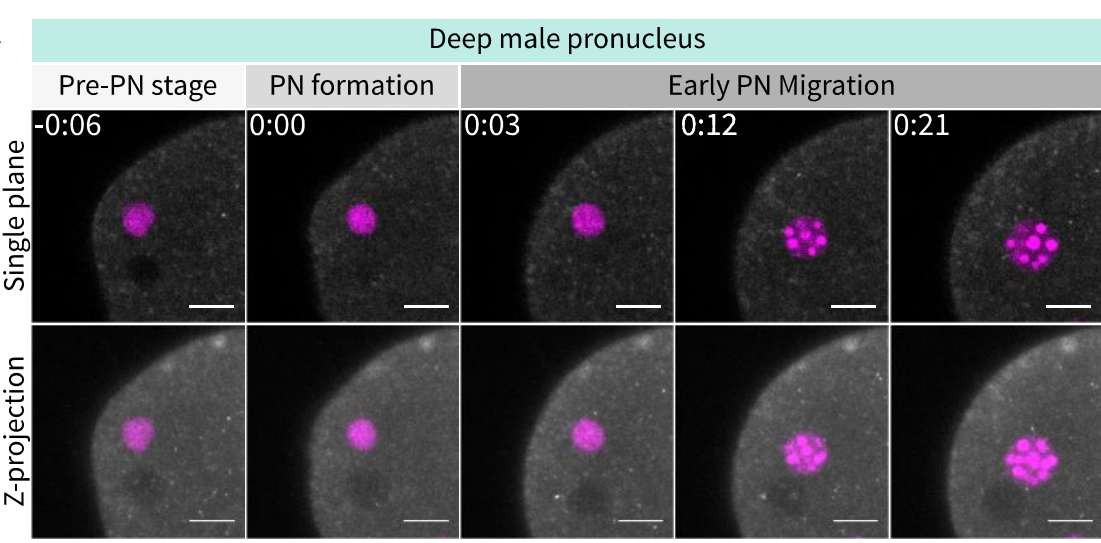

$\square$ H2B-mEGFP $\square$ mScarlet-Rab11a

D

mScarlet-Rab11a Enrichment seen at pronucleus

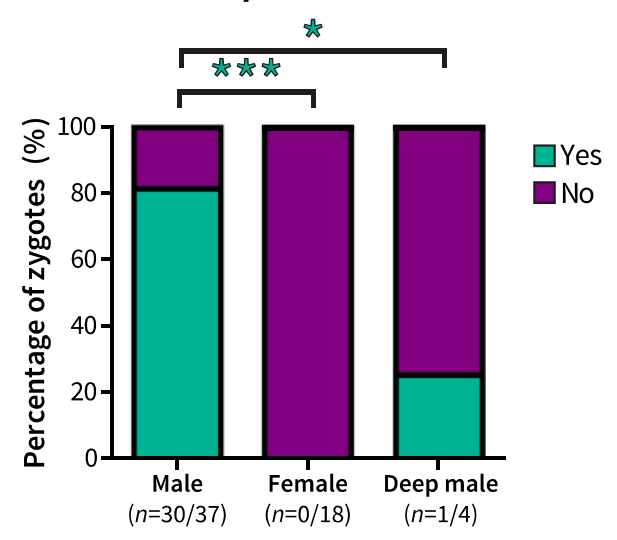


Figure 13: Live imaging of mScarlet-Rab11a in high temporospatial resolution at male and female pronuclei. (A) Confocal images of area surrounding male pronucleus forming close to the cortex of live zygote expressing mScarlet-Rab11a (grey) and H2B-mEGFP (magenta) over time. Single z-planes show plane at which male DNA is central; z-projection is of $15 \times 1.5 \mu \mathrm{m}$ around centre. Cyan arrow indicates Rab11a accumulation as the pronucleus forms adjacent to the cortex. Images taken with a Zeiss LSM-800 microscope. Gaussian blur $1 \mathrm{px}$ applied. Scale bar $=10 \mu \mathrm{m}$. Time shown in hours:minutes relative to pronuclear formation. Representative images from a total of 4 experiments are shown, total $n=37$. (B) Confocal images of area surrounding female pronucleus forming away from the cortex of live zygote expressing mScarlet-Rab11a (grey) and H2BmEGFP (magenta) over time. Single z-planes show plane at which female DNA is central; z-projection is of $15 \times 1.5 \mu m$ around centre. The last time point before pronuclear formation is set as time point 0:00. Images taken with a Zeiss LSM-800 microscope. Gaussian blur 1 px applied. $P B=$ Polar body; Scale bar $=10 \mu \mathrm{m}$. Time shown in hours:minutes. Representative images from a total of 4 experiments are shown, total $n=18$. (C) Confocal images of area surrounding a male pronucleus forming further away from the cortex of live zygote expressing mScarlet-Rab11a (grey) and H2B-mEGFP (magenta) over time. Single z-planes show plane at which male DNA is central; z-projection is of $15 \times 1.5 \mu \mathrm{m}$ around centre. Images taken with a Zeiss LSM-800 microscope. Gaussian blur 1 px applied. Scale bar $=10 \mu \mathrm{m}$. Time shown in hours:minutes relative to pronuclear formation. Representative images from one experiment are shown, $n=4$. (D) Quantification of enrichment seen at male pronuclei shown in (A), female pronuclei shown in (B), and deep male pronuclei shown in (C). Presence of mScarlet-Rab11a enrichment was judged by eye, three separate times per zygote to avoid bias, with a yes/no read-out. Two-tailed Fisher's exact test used to test statistical significance between Male pronucleus group and the other two groups individually; * $p \leq 0.05$, $* * p \leq 0.01, * * * p \leq 0.001$. 


\section{Live imaging of mScarlet-Rab11a at the male pronucleus with Rab11a perturbations}

With the newly optimised settings for high temporospatial imaging of the forming male pronucleus, I next wanted to investigate whether this mScarlet-Rab11a enrichment would be affected by perturbation by brefeldin A and expression of SNAP-Rab11a ${ }^{S 25 N}$ mRNA, like the cytoplasmic Rab11apostive vesicles detected by immunofluorescence. These results are shown in Figure 14. Zygotes expressing mScarlet-Rab11a and H2B-mEGFP mRNA, which were either incubated in methanol/brefeldin A or expressing SNAP-Rab11a ${ }^{W T} / S N A P-R a b 11 a^{S 25 N}$ mRNA, were imaged with a focus on the male pronucleus forming at the cortex.

In Figure 14A, the methanol-treated zygote showed mScarlet-Rab11a enrichment at the fertilisation cone and adjacent to the forming male pronucleus, in particular at time point $0: 12$, as indicated by the cyan arrow. The male pronucleus migrated away from the cortex and the mScarlet-Rab11a enrichment faded. In the example shown of the brefeldin A-treated zygote, the male pronucleus clearly formed, but migrated much slower away from the cortex, and even 30 minutes after pronuclear formation, the male pronucleus was larger but remained at the cortex. This reflected what was observed in the pronuclear migration tracking experiments using brefeldin A by Kathleen Scheffler (see Introduction 1.4). No mScarlet-Rab11a enrichment was seen as the male pronucleus forms, indicating that this perturbation also blocked the formation of this accumulation. However, as brefeldin A blocks coat recruitment at the Trans-Golgi network, other cellular structures were also affected, and fluorescent clusters such as those pointed out by the magenta arrow were seen throughout the cytoplasm and near the cortex in all zygotes imaged. In many cases, this made it even more difficult to analyse by eye whether mScarlet-Rab11a enrichment was present. For this reason, this experiment was not quantified, and also not repeated.

In Figure 14B, the results of this imaging when expressing either SNAP-Rab11 $a^{W T}$ or Rab11 $a^{S 25 N}$ mRNA are shown. In the presence of SNAP-Rab11a ${ }^{\mathrm{WT}}$, a faint accumulation of mScarlet-Rab11a was seen, indicated by the cyan arrow. However, this was fainter than in previous experiments, likely because of the co-expression and resulting co-localisation of the fluorescently tagged mScarlet-Rab11a and the non-fluorescently-tagged SNAP-Rab11a ${ }^{\mathrm{WT}}$, on top of the endogenous protein. In the zygote expressing the dominant-negative version of Rab11a, no accumulation of Rab11a could be seen, indicating that this perturbation of Rab11a also blocked this accumulation. However, as in this case it was very difficult to see the accumulation in wild-type-expressing cells, also this experiment was not quantified nor repeated.

These preliminary experiments did indicate that perturbations of Rab11a likely disrupted the accumulation of mScarlet-Rab11a seen at the cortex as the pronucleus formed, as well as perturbing 
the fast, initial migration of the male pronucleus away from the cortex. However, as quantification of the Rab11a accumulation was already difficult under normal conditions, and the clusters caused by brefeldin $A$ as well as the co-expression of SNAP-Rab11a ${ }^{W T}$ made objective quantification of this enrichment even more difficult, these experiments were not reproduced further. Instead, actinrelated proteins linked to Rab11a vesicles in oocytes, Spire2 and Formin-2, were investigated in the next experiments.

A

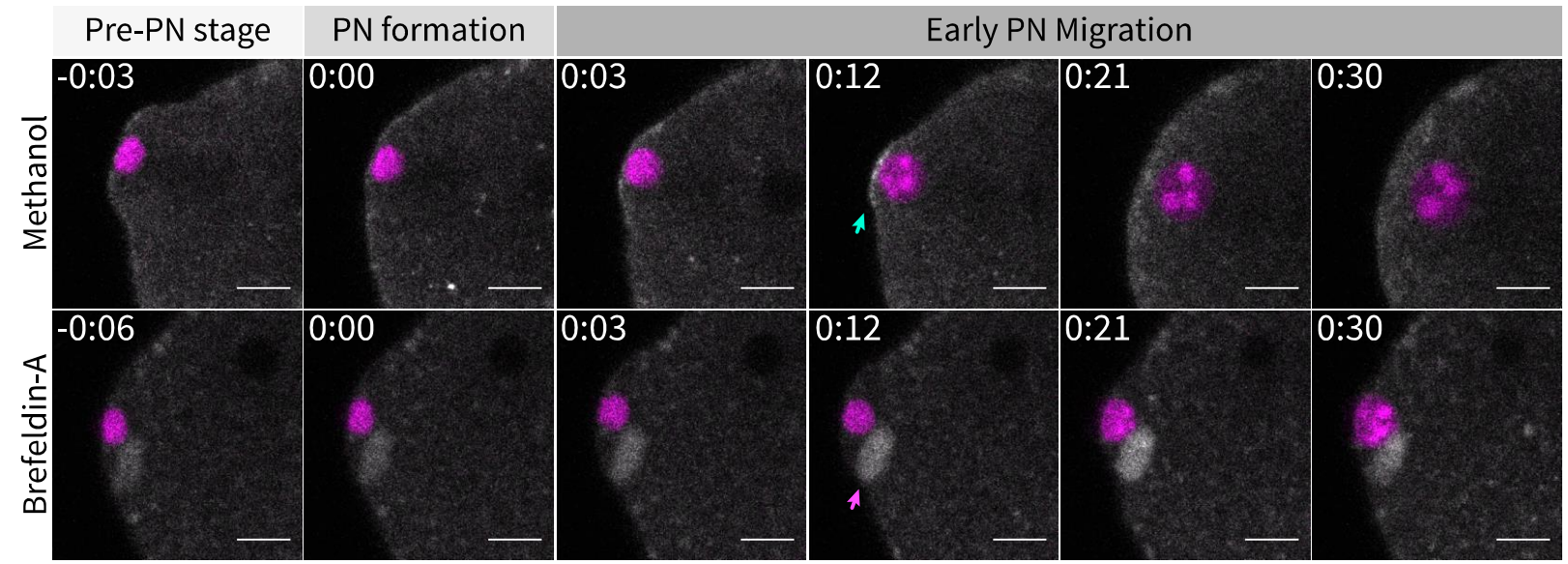

$\square$ H2B-mEGFP $\square$ mScarlet-Rab11a

B

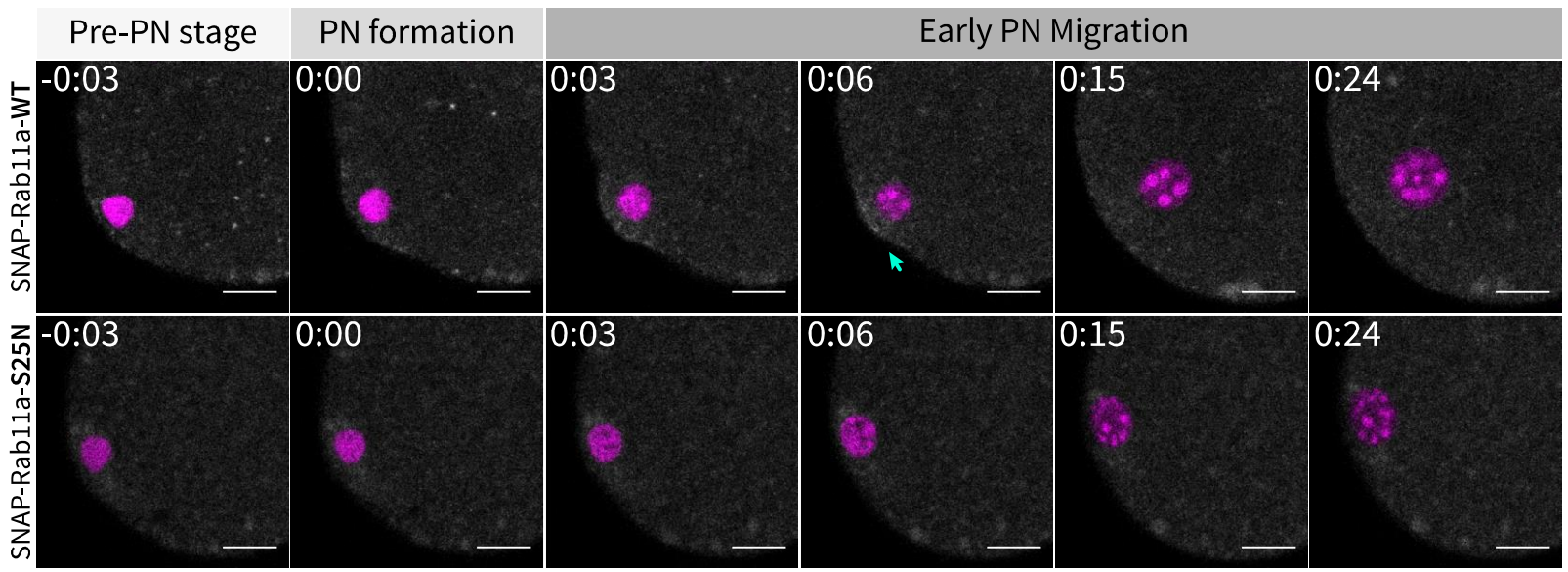

$\square$ H2B-mEGFP $\square$ mScarlet-Rab11a

Figure 14: Live imaging of $\mathrm{mScarlet-Rab11a}$ at the male pronucleus with Rab11a perturbations. (A) Confocal images male pronucleus forming close to the cortex of live zygote expressing mScarlet-Rab11a (grey) and H2B-mEGFP (magenta), treated with either methanol (top) or brefeldin A (bottom), over time. Single z-planes of plane at which male DNA is central is shown. Cyan arrow indicates Rab11a accumulation as the pronucleus forms adjacent to the cortex; magenta arrow indicates cluster in cytoplasm. Images taken with a Zeiss LSM-800 microscope. Gaussian blur 1 px applied. Scale bar $=10 \mu m$. Time shown in hours:minutes relative to pronuclear formation. Representative images from one experiment are shown, with $\mathrm{n}=8$ for methanol, and $\mathrm{n}=10$ for brefeldin $A$. (B) Confocal images male pronucleus forming close to the cortex of live zygote expressing mScarlet-Rab11a (grey) and H2B-mEGFP (magenta), also expressing either SNAP-Rab11aWT (top) or SNAP-Rab11a ${ }^{\mathrm{S} 25 \mathrm{~N}} m R{ }^{\mathrm{WNA}}$ (bottom), over time. Single z-planes of plane at which male DNA is central is shown. Cyan arrow indicates Rab11a accumulation as the pronucleus forms adjacent to the cortex. Images taken with a Zeiss LSM-800 microscope. Gaussian blur $1 \mathrm{px}$ applied. Scale bar $=10 \mu \mathrm{m}$. Time shown in hours:minutes relative to pronuclear formation. Representative images from one experiment are shown, with $\mathrm{n}=8$ for SNAP-Rab11aWT, and $\mathrm{n}=8$ for SNAP-Rab11a ${ }^{\mathrm{S} 25 \mathrm{~N}}$. 


\section{Optimisation of Spire2 and Formin-2 live imaging in the mouse zygote}

To further investigate the role of the Rab11a in pronuclear migration, the localisation of two actin nucleators related to Rab11a in oocytes, Spire2 and Formin-2, was investigated. First, live imaging of mEGFP-Spire2, mCherry-Spire2, and Formin-2-EGFP was tested in zygotes imaged every 15 minutes and with a z-resolution of $3 \mu \mathrm{m}$. The results of this are shown in Figure 15.

First, expression of mEGFP-Spire2 mRNA on its own was tested. As seen in Figure 15A, when mEGFPSpire 2 mRNA with a concentration of $150 \mathrm{ng} / \mu \mathrm{L}$ was expressed in the zygote, it showed only very faint cytoplasmic localisation in the early zygote. However, as the male pronucleus began to form, a bright spot of mEGFP-Spire2 was detected at the cortex next to it, seen at 0:30 and 0:45 and indicated by the cyan arrows. This spot disappeared by one hour post pronuclear formation, and the cytoplasmic levels of Spire2 increased until nuclear envelope breakdown.

Next, Formin-2-EGFP was imaged. I initially imaged this on its own in the zygote, but could not see any specific localisation over time (data not shown). As it was previously shown that Formin-2-EGFP colocalised with mCherry-Spire2 in mouse oocytes (Pfender et al., 2011), I next attempted to co-express them, as seen in Figure 15B. While the Formin-2-EGFP mRNA concentration was kept the same in all experiments, two different mCherry-Spire2 mRNA concentrations were used, to see if higher overexpression would change the Formin-2-EGFP localisation. To see the location of the DNA as well, the zygotes were incubated with SiR-DNA during imaging; note that this allowed only weak visualisation of the DNA before pronuclear formation, and once the pronuclei had formed and migrated inwards, SiR-DNA labelling was too weak to see them. The signal was not sufficient to determine the exact timing of pronuclear formation, and thus, the time is given from the start of imaging; however, it was enough to determine the approximate location of pronuclear formation of the male pronucleus, annotated in the figure.

In zygotes with a lower mCherry-Spire2 mRNA concentration $(150 \mathrm{ng} / \mu \mathrm{L})$, mCherry-Spire2 accumulation at the cortex above the male pronucleus was observed, as pointed out by the cyan arrow in the top panels of Figure 15B. The accumulation was present from 15 minutes after start of imaging, until time point 2:45. This is longer than observed in Figure 15A, which may have been due to coexpression, or the differences in mRNA stock, the fluorophore, and/or imaging settings. However, no specific Formin-2-EGFP localisation was observed. When mCherry-Spire2 mRNA was expressed at a higher concentration of $400 \mathrm{ng} / \mu \mathrm{L}$, this mCherry-Spire2 accumulation stayed at the cortex even longer, with a ring-like structure appearing around the area of the sperm entry site prior to pronuclear formation, which then appeared to constrict into a spot. This spot-like accumulation stayed visible until 4:45. In these zygotes, Formin-2-EGFP was observed to accumulate in the same area as mCherry- 
Spire2, appearing from time point 1:15 in the area where the male pronucleus had formed. It remained there over eight hours, until the end of imaging.

These experiments indicated that Spire2, tagged with either mCherry or mEGFP, could localise to the cortical area adjacent to the forming male pronucleus, to the same area that mScarlet-Rab11a accumulation had been observed. It also showed that Spire 2 mRNA overexpression could alter Formin2-EGFP localisation, as well as the duration of Spire2 accumulation at the cortex.

These results were used to further optimise the next experiments. I did not continue to focus on Formin-2, as it required co-expression with high levels of Spire2 to show a detectable, specific signal. However, Kathleen Scheffler used these preliminary experiments to later image Formin-2 in more detail at the forming male pronucleus, in co-expression with Spire2. These data are shown in Figure 29 in the appendix. I, on the other hand, continued to study Spire2 localisation in the next experiments. My next step was to co-express mEGFP-mSpire2 with mScarlet-Rab11a mRNA to investigate their co-localisation. 
A

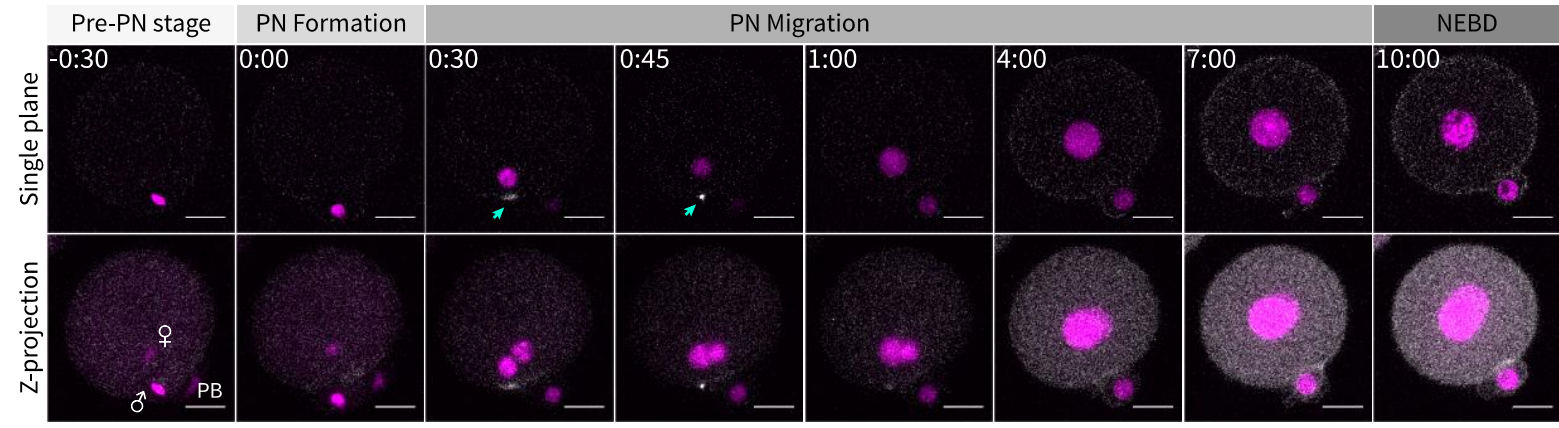

B

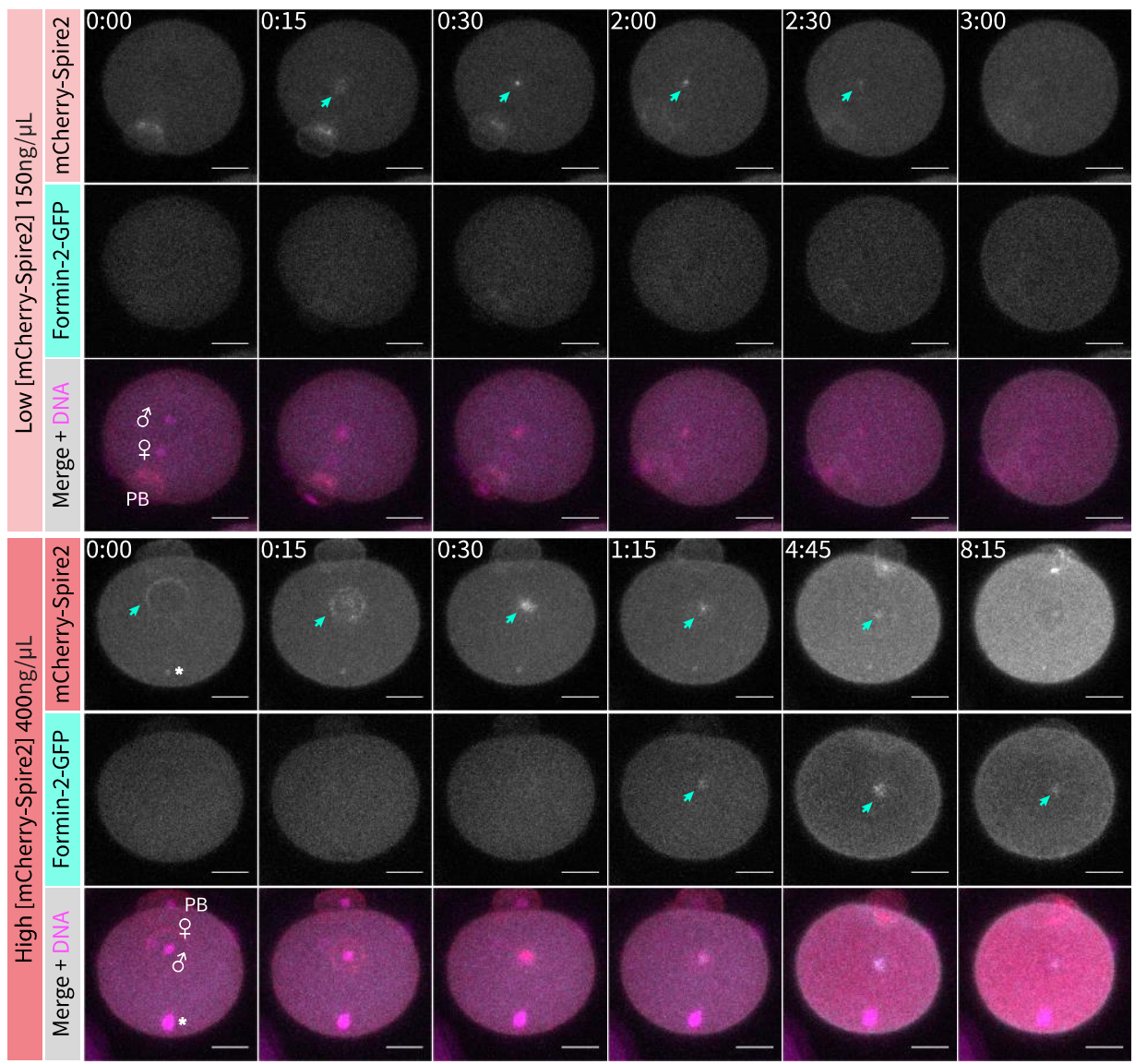

$\square$ mCherry-Spire2 $\square$ Formin-2-EGFP $\square$ SiR-DNA

Figure 15: Optimisation of mEGFP-Spire2, mCherry-Spire2, and Formin-2-EGFP live imaging in mouse zygotes. (A) Confocal images of zygotes expressing mEGFP-Spire2 (grey) and H2B-mCherry mRNA (magenta), over time. Zygotes were imaged every 15 minutes, with a z-resolution of $3 \mu \mathrm{m}$; single plane shows central plane of male pronucleus, and z-projection shows maximum projection of $28 \times 3 \mu \mathrm{m}$. For both, Gaussian blur of $1 \mathrm{px}$ was applied. $P B=$ Polar body, $\sigma^{x}=$ male pronucleus $q=$ female pronucleus. Cyan arrow indicates mEGFP-Spire2 accumulation. Images taken with a Zeiss LSM-880 microscope. Gaussian blur $1 \mathrm{px}$ applied. Scale bar $=20 \mu \mathrm{m}$. Time shown in hours:minutes relative to pronuclear formation. Representative images from one experiment, $\mathrm{n}=9$ zygotes. (B) Co-expression of Formin-2-EGFP (grey in central panels; cyan in merged panels) with low (1:16 dilution) and high (1:6 dilution) of mCherry-Spire2 mRNA (grey in top panels; red in merged panels) in zygotes incubated with SiR-DNA (magenta in merged panels). z-projections of $28 \times 3 \mu \mathrm{m}$ are shown with a Gaussian blur of 1 px applied. $P B=$ Polar body, $\sigma^{7}=$ male pronucleus $q=$ female pronucleus. Cyan arrow indicates mCherry-Spire 2 or Formin-2-EGFP accumulation. Images taken with a Zeiss LSM-880 microscope. Gaussian blur 1 px applied. Scale bar $=20 \mu m$. Time shown in hours:minutes relative to start of imaging. Representative images from one experiment, $\mathrm{n}=11$ for $1: 16$ dilution and $\mathrm{n}=10$ for 1:6 dilution. 


\section{Co-expression of Rab11a and Spire2 at the male pronucleus}

As fluorescently tagged Spire2 localised to the cortical region by the forming male pronucleus, I wanted to compare the pattern and timing of mScarlet-Rab11a and mEGFP-Spire2 enrichment in this area by co-expressing their mRNA and imaging the area surrounding the male pronucleus. The results of this are shown in Figure 16. Zygotes expressing mScarlet-Rab11a and mEGFP-Spire2 were imaged, zoomed-in at the area of the fertilisation cone. In this case, no DNA marker was used. This was because the same experiment was first attempted with SiR-DNA, which has a far-red fluorophore, and the filter/laser settings for the channel excited by the $561 \mathrm{~nm}$ laser had to be set so narrowly to avoid cross-talk between the red- and far-red channels, that the mScarlet-Rab11a signal was very weak and barely visible above the noise (data not shown). Therefore, SiR-DNA was not added in the shown experiment, and the position was set based on locating the sperm entry site in the transmitted light image. Note that the time given is relative to the start of imaging, as opposed to the time of pronuclear formation, as the timing of pronuclear formation could not be clearly determined.

The mEGFP-Spire2 signal was quite noisy, as shown in the upper panels of Figure 16; however, a clear enrichment was seen from 0:24, indicated by the cyan arrow, which soon formed a bright spot seen at 0:33 and 0:42, which was not visible anymore by 1:06. This bright spot had a similar appearance as the enrichment seen in the mEGFP-Spire2 signal shown in Figure 15, where a bright spot was also observed next to the forming male pronucleus.

In the panels of Figure 16 showing mScarlet-Rab11a, an initial faint signal was seen over the fertilisation cone, similar to what was seen in Figure 13, indicated by the cyan arrow at 0:12. As the pronucleus appeared to form below this area, the signal became brighter and more localised, as seen at 0:24. This, again, was similar to the Rab11a enrichment described previously in figure 7 and 13 . However, the mScarlet-Rab11a enrichment previously described in Figure 13 faded after this point; in co-expression with mEGFP-Spire2 shown here, the mScarlet-Rab11a signal did not fade, and instead too formed a bright spot at 0:33 and 0:42. Note that imaging settings were checked to confirm this was not due to a cross-talk between channels - the two channels were imaged on separate tracks.

From this experiment, two conclusions could be drawn: first, that mEGFP-Spire2 was not optimal for imaging, as it gave a very noisy signal; and second, that co-expression of mEGFP-Spire2 appeared to alter the localisation of mScarlet-Rab11a. However, it was not clear whether there was also an effect in the other direction, namely that mScarlet-Rab11a expression altered the localisation of mEGFPSpire2, partly because the mEGFP signal was not very clear. Due to the effect of co-expression, these results did not allow a real comparison of Spire2 and Rab11a localisation. Therefore, our next step was to find a better fluorophore with which to express Spire2, and to focus on visualising this on its own. 


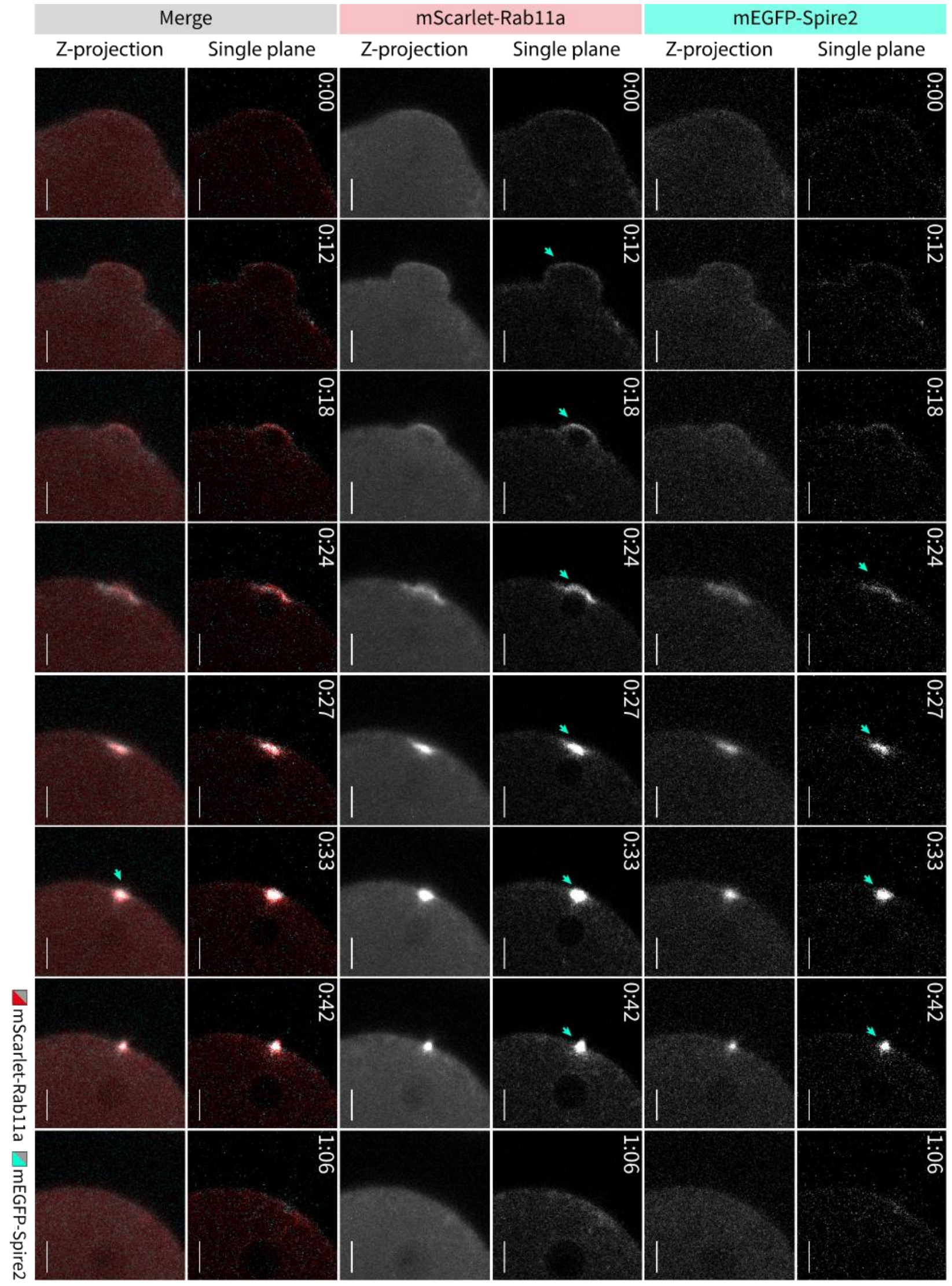


Figure 16: Co-expression of mScarlet-Rab11a and EGFP-Spire2 at the male pronucleus. Confocal images of zygotes coexpressing mEGFP-Spire2 (grey in top panels; cyan in merged panels) with mScarlet-Rab11a mRNA (grey in middle panels; red in merged panels), zoomed into the fertilisation cone area of the zygote. Single planes show the middle plane of the fertilisation cone or of the accumulation signal later; z-projections of $15 \times 1.5 \mu \mathrm{m}$ are shown below single planes; Gaussian blur of $1 \mathrm{px}$ applied to all images. Cyan arrow indicates mEGFP-Spire2 or mScarlet-Rab11a accumulation. Images taken with a Zeiss LSM-800 microscope. Scale bar $=10 \mu \mathrm{m}$. Time shown in hours:minutes relative to start of imaging. Representative images from one experiment, $\mathrm{n}=4$.

\section{Live imaging of mClover3-Spire2 in high temporospatial resolution at the forming pronuclei}

To achieve better live imaging results of Spire2, I constructed the plasmid pGEMHE-mClover3-Spire2 for in vitro expression of mClover3-Spire2 mRNA; see Methods 2.1 for cloning strategy. Based on my previous imaging of mEGFP-Spire2 and mCherry-Spire2 shown in Figure 15, as well as the higher resolution live imaging optimised with mScarlet-Rab11a shown in Figure 13, this mRNA was then used for high temporospatial resolution imaging of male and female pronuclei by Kathleen Scheffler. As these experiments were the result of a collaborative effort, and the results are highly relevant to experiments I will show later, I have included them here in the results section. While I initially helped with the set-up of the imaging to be similar as the mScarlet-Rab11a imaging shown in Figure 13 as possible, the data shown in Figure 17 were collected by Kathleen Scheffler.

In Figure 17A, confocal images of zygotes expressing mClover3-Spire2 and H2B-mCherry mRNA are shown, with both single planes at the central plane of the forming male pronucleus shown above, and z-projection of $15 \times 1.5 \mu \mathrm{m}$ shown below. Nine minutes before pronuclear formation, a faint mClover3Spire2 signal could be observed at the edges of the fertilisation cone, indicated by the cyan arrows. In the z-projection, this localisation appeared almost like a ring surrounding the fertilisation cone, which 'contracted' or became smaller as the pronucleus formed and moved away from the cortex, eventually leaving a bright spot in the cortex. It is clear that the mClover3 signal was much brighter and clearer than the mEGFP signal shown previously. Also, as this signal covered a larger area and the accumulation became very bright, it was also easier to quantify its presence than mScarlet-Rab11a, even if the zygote slightly rotated or the angle was not perfect.

A few female pronuclei were also imaged, to confirm if no such bright accumulation could be seen at their formation, like for mScarlet-Rab11a. These results are seen in Figure 17B. Some bright spots were visible at the edge of the polar body, likely as Spire2 is known to be involved in polar body extrusion. However, as the female pronucleus formed, no clear increase in signal or accumulation was seen, whether in the cortical region overlying it or the adjacent cytoplasm.

The quantification of how often this accumulation was seen is shown in Figure 17C. As mentioned, this signal was brighter and more obvious to the human eye than the mScarlet-Rab11a signal, and $37 / 37(100 \%)$ of zygotes imaged in four separate experiments showed accumulation at the male 
pronucleus. For the four female pronuclei imaged in two of these experiments, none showed any accumulation ( $p<0.001$, two-tailed Fisher's exact test).

Now that we had seen localisation of fluorescently labelled actin-related Rab11a vesicles and the actin nucleator Spire2, the next experiments investigated whether actin nucleation itself was actually increased in this area, by live imaging different actin probes in mouse zygotes. 


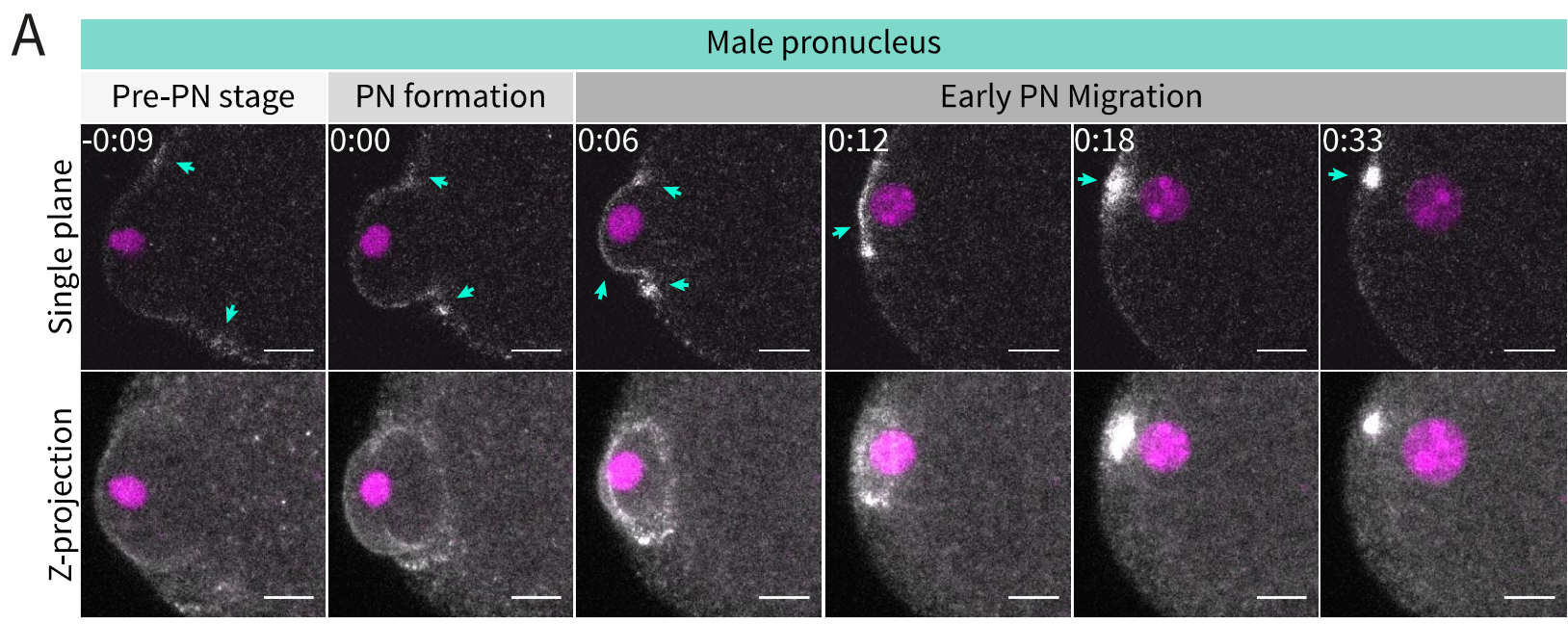

$\square$ H2B-mCherry $\square$ mClover3-Spire2

B

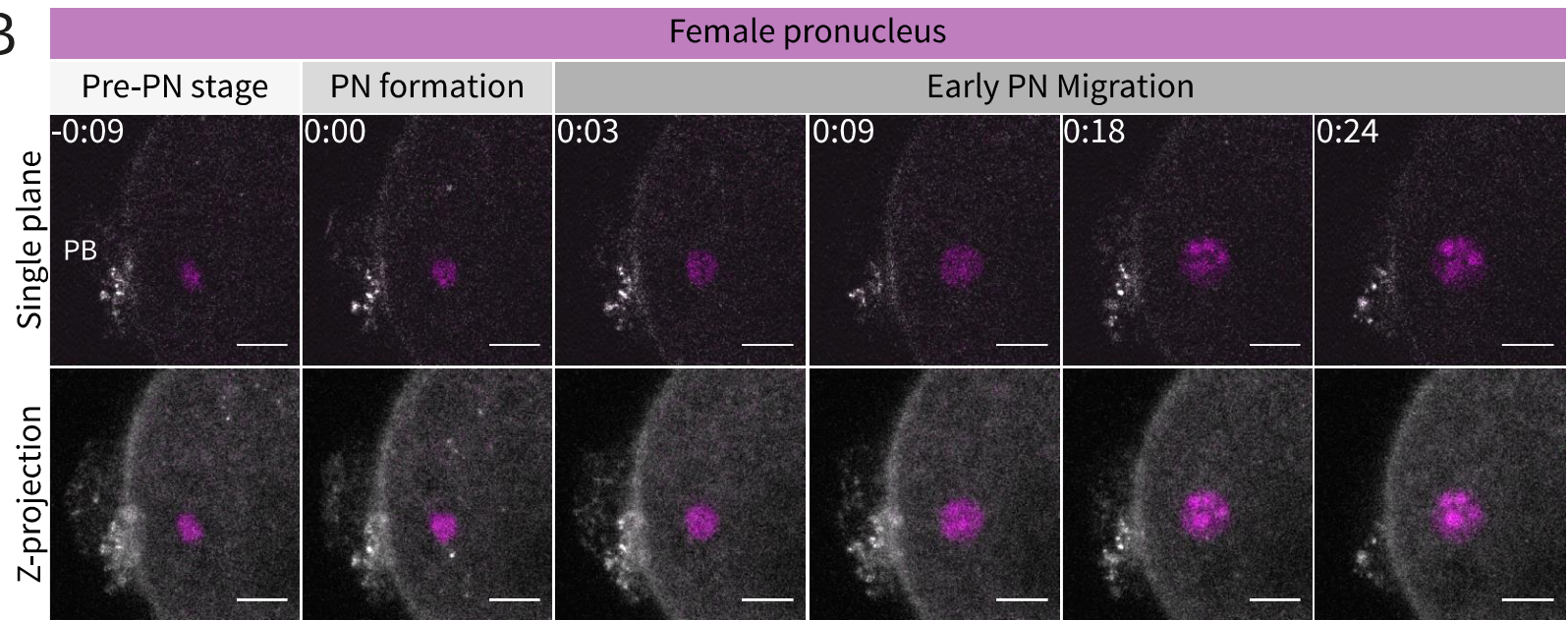

$\square$ H2B-mCherry $\square$ mClover3-Spire2

\section{C mClover3-Spire2 Enrichment seen at pronucleus}

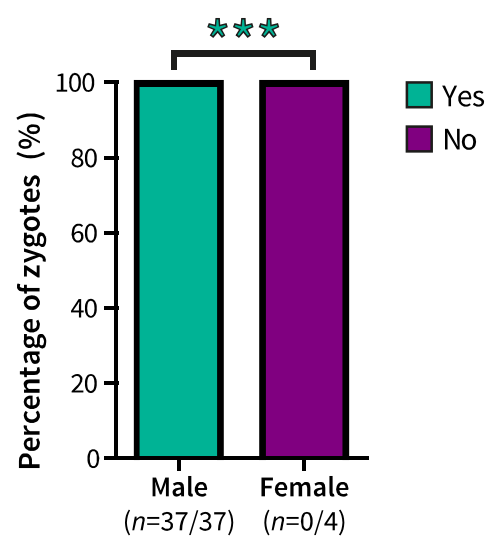

Figure 17: Live imaging of $m$ Clover3-Spire2 in high temporospatial resolution at male and female pronuclei. (A) Confocal images of area surrounding male pronucleus forming close to the cortex of live zygote expressing $m$ Clover3-Spire2 (grey) and H2B-mCherry (magenta) over time. Single z-planes show plane at which male DNA is central; z-projection is of $15 \times 1.5 \mu \mathrm{m}$ around centre. The last time point before pronuclear formation is set as time point 0:00. Cyan arrows indicate Spire2 accumulation as the pronucleus forms adjacent to the cortex. Images taken with a Zeiss LSM-880 microscope. Scale bar = $10 \mu \mathrm{m}$. Representative images from a total of 4 experiments are shown, total $n=37$ zygotes. (B) Confocal images of area surrounding female pronucleus forming away from the cortex of live zygote expressing $m$ Clover3-Spire2 (grey) and $\mathrm{H} 2 \mathrm{~B}$ - 
$m$ Cherry (magenta) over time. Single z-planes show plane at which female DNA is central; z-projection is of $15 \times 1.5 \mu \mathrm{m}$ around centre. The last time point before pronuclear formation is set as time point 0:00. Images taken with a Zeiss LSM-880 microscope. $\mathrm{PB}=$ Polar body; Scale bar $=10 \mu \mathrm{m}$. Representative images from a total of 2 experiments are shown, total $n=4$ zygotes. (C) Quantification of enrichment seen at male pronuclei shown in (A), and female pronuclei shown in (B). Presence of mClover3-Spire2 enrichment was judged by eye with a yes/no read-out. Two-tailed Fisher's exact test used to test statistical significance; $* \leq 0.05, * * p \leq 0.01, * * * p \leq 0.001$. 


\subsection{The functional role of actin nucleation on the fast migration of the male pronucleus}

\section{Optimisation of actin live imaging in the mouse zygote}

To investigate whether the Rab11a- and Spire2- accumulations seen by the forming male pronucleus may be nucleating actin filaments that play a functional role in its fast migration away from the cortex, I next tested different live actin probes in mouse zygotes and oocytes. It was known that actin is present in the fertilisation cone of the freshly fertilised zygote, and that there are actomyosin contractions in the fertilisation cone (Ajduk et al., 2011). However, it was not clear whether there could be a short-term increase in actin nucleation as a result of the acute, localised accumulation of actin-related vesicles and actin nucleators described above. Therefore, my goal was to attempt imaging of actin filaments with very high temporal resolution.

The probes tested were EGFP-tagged Utrophin, a dystrophin-related, actin-binding protein (Winder et al., 1995), which had previously been used to visualise actin in live oocytes (Schuh and Ellenberg, 2008); mClover3-tagged Utrophin, cloned by Nick Chun So in the lab; SiR-actin, a jasplakinolide-based live actin probe from the company Spirochrome; and GFP-tagged Actin Chromobody from the company Chromotek, the coding region of which I cloned into a pGEMHE vector for in vitro mRNA synthesis (see Methods 2.1 for cloning strategy). The full details of these probes can be found in Table 3 of the Methods section. The zygotes (in the case of both Utrophin probes) or GV oocytes (in the case of SiR-actin and the actin chromobody) were imaged not using standard confocal imaging, but rather using Airyscan imaging on the Zeiss LSM800 or LSM880 microscopes to better visualise individual actin filaments. These results are shown in Figure 18.

The first probe tested was EGFP-Utrophin (EGFP-UtrCH), which was tested in two separate experiments, shown in Figure 18A. In the first experiment, different concentrations and laser settings were tested, while the second experiment looked at imaging of the same cell over time to see if live imaging was possible. Four different cells are shown of the first experiment; two with a concentration of $800 \mathrm{ng} / \mu \mathrm{L}$ of the EGFP-UtrCH mRNA, and one each of $320 \mathrm{ng} / \mu \mathrm{L}$ and $200 \mathrm{ng} / \mu \mathrm{L}$. The first image of the $800 \mathrm{ng} / \mu \mathrm{L}$ concentration group shows the area surrounding the male pronucleus shortly after it had formed and began migrating away from the cortex. Overall, actin filaments were clearly visible in the cytoplasm of the cell. A bright EGFP-Utrophin enrichment was seen in the fertilisation cone, as well as some spots between the cone and the nucleus, as indicated by the cyan arrows. The area overlaying the female pronucleus, as shown in the next image, did not have such an enrichment in the $800 \mathrm{ng} / \mu \mathrm{L}$ concentration group. However, one issue with EGFP-Utrophin is that it can lead to cytoskeletal defects as it binds endogenous F-actin, an example of which is shown in the next image, where a $320 \mathrm{ng} / \mu \mathrm{L}$ concentration of the mRNA was expressed. The cyan arrow indicates some EGFP- 
Utrophin-positive blebs in the cytoplasm near the male pronucleus, as well as some bright spots near the cortex. With this lower concentration of EGFP-Utrophin mRNA, the cortical signal appeared weaker, and the cytoplasmic actin filaments were not as clear, though still visible. To see if lower concentrations could avoid such side effects while still allowing visualisation of actin filaments, a concentration of $200 \mathrm{ng} / \mu \mathrm{L}$ was also tested. While the fertilisation cone and cortical actin were clearly visible, no clear filaments could be seen in the cytoplasm, even with a higher laser power of $5 \%$ compared to $1.5-2 \%$ laser power used for the previous images.

Even though EGFP-Utrophin caused some side effects in the cell, I next wanted to test if live imaging with a high temporal resolution was possible, to see if we could detect the dynamics of the actin cytoskeleton as the male pronucleus migrated away from the cortex. For this, a single zygote expressing EGFP-Utrophin mRNA with a concentration of $400 \mathrm{ng} / \mu \mathrm{L}$ was imaged in the second experiment. An Airyscan image was taken every 1.26 seconds, with the first, $21^{\text {st }}$ and $41^{\text {st }}$ time points shown in. While at first, the actin cortex, some filaments and actin nodes indicated by the cyan arrows were visible and highly dynamic, the fluorophore appeared to quickly get bleached by the laser, as within less than 30 seconds, the signal had weakened; see cortex in bottom left of image. However, this temporal resolution was required to capture the dynamics of the actin cytoskeleton as done previously (Holubcová et al., 2013), and without such high laser powers, the filaments and nodes were not visible. Therefore, I decided to try Utrophin tagged with a different fluorophore, mClover3, next. Images of representative zygotes expressing mClover3-Utrophin are shown in Figure 18B. The area around the fertilisation cone from a single zygote expressing mClover3-Utrophin mRNA with a concentration of $400 \mathrm{ng} / \mu \mathrm{L}$ was imaged every 1.25 seconds; time points 1, 21, 41, and 61 are shown. While the mClover3 fluorophore required lower laser powers than EGFP, and some cytoplasmic structures could be identified in the first image, the fluorophore was quickly bleached in this time series. It should be noted that zygotes expressing $800 \mathrm{ng} / \mu \mathrm{L}$ and $200 \mathrm{ng} / \mu \mathrm{L}$ dilutions of this mRNA were also imaged, but did not show anything different, and are therefore not shown here.

As Utrophin was found to cause some side effects when expressed in high concentrations, did not allow visualisation of filaments when expressed in lower concentrations, and was easily bleached whether EGFP- or mClover3-tagged, I decided to try Airyscan imaging of alternative actin probes next. For these initial tests, I used GV oocytes instead of zygotes, as they did not require superovulation to retrieve.

The first alternative actin probe I tried was SiR-actin, a far-red probe based on the actin-inhibiting drug jasplakinolide. SiR-actin is supposed to be cell-permeable, but incubation with SiR-actin was not sufficient to allow observation of any actin structures in GV oocytes (data not shown). Therefore, SiR- 
actin was microinjected directly into the cytoplasm of GV oocytes at different concentrations; undiluted $(1 \mathrm{mM}), 40 \mu \mathrm{M}$, and $4 \mu \mathrm{M}$. These results are shown in Figure 18C. First, two GV oocytes are shown, into which undiluted SiR-actin was injected. While a signal was seen at the cortex and in the cytoplasm, in both cells, a large area of the cytoplasm showed a sort of 'frozen' cytoskeleton as well as large, bright nodules unevenly distributed. What was also apparent was that the side on which the injection had occurred had brighter staining at the cortex. Clearly, undiluted SiR-actin injected straight into the cytoplasm caused defects in the cell. An injection of SiR-actin at a concentration of $40 \mu \mathrm{M}$ did not cause any obvious defects, as shown in the next two cells. However, it also did not allow clear visualisation of the actin filaments inside the cytoplasm, even with laser powers as high as $2-4 \%$. The actin cortex was clearly visible, but in both cells, the signal appeared stronger on the side at which the injection had taken place. This indicated that the SiR-actin probe was not distributed evenly in the cell upon microinjection. Lastly, SiR-actin with a concentration of $4 \mu \mathrm{M}$ was injected into the cytoplasm of a GV oocyte. As can be seen in this Airyscan image, no cytoplasmic filaments were visible; however, the weaker dilution also did not cause an uneven staining of the clear actin cortex. Unfortunately, as we required a probe for fast imaging of small F-actin structures without perturbing the architecture of the cell, SiR-actin was not a feasible solution.

The last actin probe tested was the Actin Chromobody, which is based on a nanobody against the actin protein, bound to a TagGFP probe. While it is intended to be transfected into tissue culture cells, oocytes are transcriptionally silent, which is why mRNA expression is used instead of transfection. For this reason, I first cloned the coding region of the Actin Chromobody plasmid into the PGEMHE vector used for in vitro mRNA synthesis. The undiluted mRNA was injected into the cytoplasm of GV oocytes and expressed for three hours, and the results shown in Figure 18D. While a signal was observed on both the cortex and in the cytoplasm in the two oocytes shown, and no clear deformation or defects were seen in the cell, the cytoplasmic signal did not show filaments, even with Airyscan microscopy. Therefore, this actin probe did not prove optimal for the requirements of imaging actin filaments in high temporal resolution.

None of the tested actin probes were found to be adequate for live-imaging of actin nucleation at the male pronucleus. While the Utrophin-based actin probes confirmed that actin was present in the fertilisation cone, and possibly showed some brighter actin filaments between the forming, migrating male pronucleus and the overlying cortex, they also caused defects in the cell and bleached too quickly to allow imaging with high temporal resolution, and thus tracking of actin filaments. The other two actin probes either created a frozen actin cytoskeleton, or did not allow visualisation of individual actin filaments. Therefore, instead of continuing imaging of actin filaments, the rest of the experiments were focused on functional as opposed to purely observational experiments. 


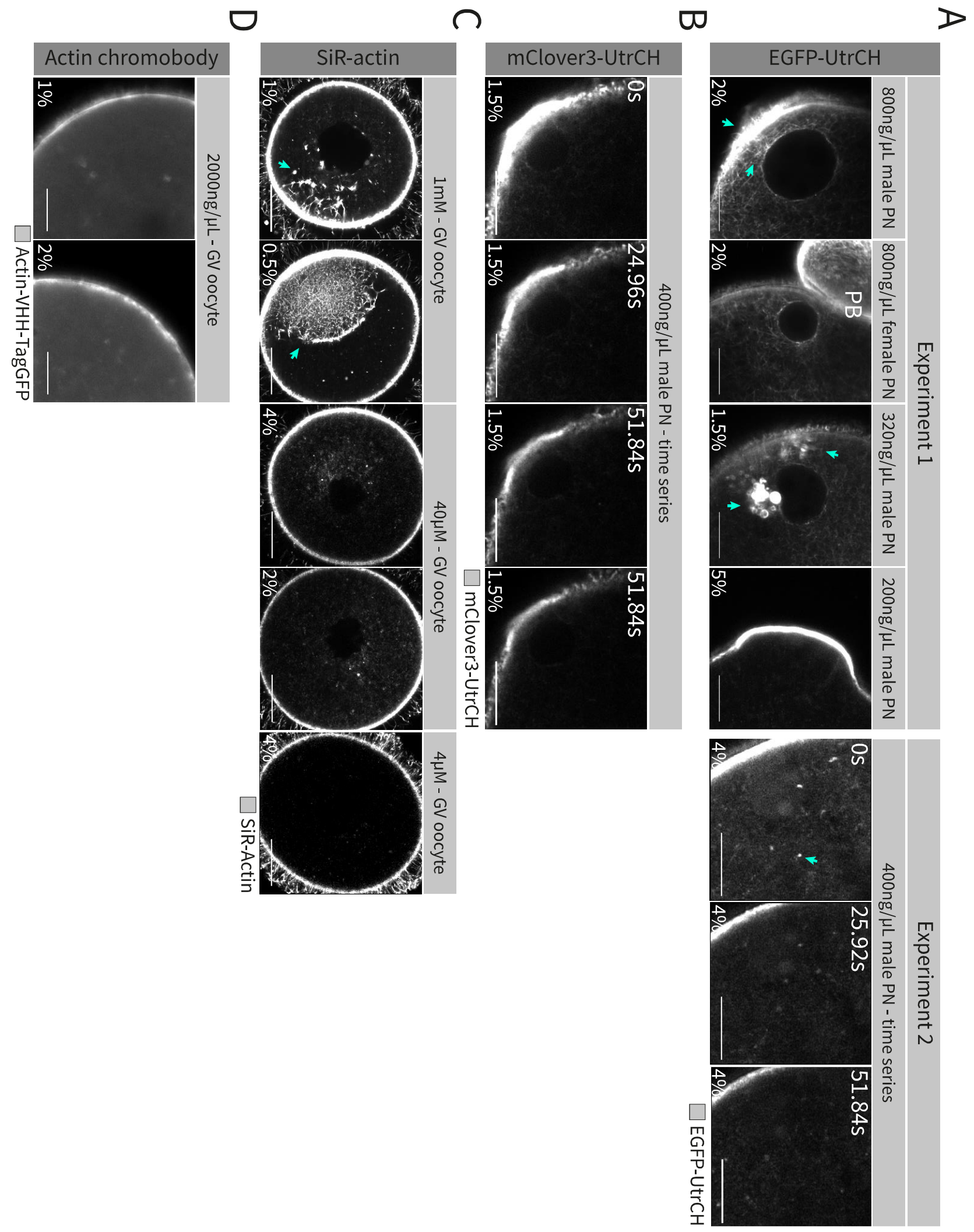

Figure 18: Optimisation of different actin probes for live imaging in mouse zygotes. (A) Airyscan images of zygotes expressing EGFP-Utrophin (EGFP-UtrCH) from two separate experiments, with different mRNA concentrations and laser settings tested. Cyan arrows indicate actin structures of interest, as well as possible defects caused by the expression of the mRNA. Only single z-planes were imaged. For the first experiment, example images are shown from four different zygotes; total number of zygotes imaged was 10. For the second experiment, example time points of one zygote imaged every $1.26 \mathrm{~s}$ are shown, time given in seconds after imaging start. A total of 13 zygotes were imaged in this experiment. Images taken with a Zeiss LSM-880 microscope in the first experiment, and LSM-800 in the second experiment, both using the Airyscan 
mode. $P B=$ Polar body; Scale bar $=10 \mu \mathrm{m}$. Percentage shown in bottom left corner indicates strength of $488 \mathrm{~nm}$ laser. (B) Airyscan images of zygote expressing mClover-Utrophin (mClover-UtrCH) over time, imaged every 1.25s with selected time points shown here, time given in seconds after imaging start. A total of 23 zygotes were imaged in this experiment, which were injected with $800 \mathrm{ng} / \mu \mathrm{L}(\mathrm{n}=7), 400 \mathrm{ng} / \mu \mathrm{L}(\mathrm{n}=9)$ and $200 \mathrm{ng} / \mu \mathrm{L}(\mathrm{n}=7)$ concentrations of the $\mathrm{mRNA}$; an example of a zygote expressing the $400 \mathrm{ng} / \mu \mathrm{L}$ mRNA is shown here. Images taken with a Zeiss LSM-800 microscope using the Airyscan mode. Scale bar $=10 \mu \mathrm{m}$. Percentage shown in bottom left corner indicates strength of 488nm laser. (C) Airyscan images of GV oocytes injected with different concentrations of the far-red SiR-actin probe. Cyan arrows indicate actin structures of interest, as well as possible defects caused by injection of the probe. Only single z-planes were imaged. A total of 6 zygotes were imaged in this experiment, 2 per concentration group, though only one of the $4 \mu \mathrm{M}$ group is shown here. Images taken with a Zeiss LSM880 microscope using the Airyscan mode. Scale bar $=10 \mu \mathrm{m}$. Percentage shown in bottom left corner indicates strength of $633 \mathrm{~nm}$ laser. (D) Airyscan images of GV oocytes expressing undiluted mRNA for the GFP-tagged Actin chromobody. Only single z-planes were imaged. Four GV oocytes were imaged in this experiment, two of which are shown here. Images taken with a Zeiss LSM-800 microscope using the Airyscan mode. Scale bar $=10 \mu \mathrm{m}$. Percentage shown in bottom left corner indicates strength of $488 \mathrm{~nm}$ laser.

\section{Spire2-Formin-2 interaction is required for fast migration of the male pronucleus}

We had previously established that Rab11a has a functional role in the fast migration of the male pronucleus away from the cortex by perturbing Rab11a with brefeldin A (see Figure 4 of the Introduction section 1.4) and Rab11a ${ }^{525 \mathrm{~N}}$ (see Figure 5). As actin nucleator Spire2 localises to Rab11apositive vesicles in oocytes (Schuh, 2013), and fluorescently tagged Rab11a and Spire2 were both found to localise to the cortical region at the male pronucleus as it formed, we next wondered if Spire2 function was also essential for the fast migration. In previous reports, Spire2 function was inhibited in RNAi-treated oocytes grown through follicular culture (Pfender et al., 2011; Schuh, 2013). As this was not an option for our superovulation and IVF protocol, we instead injected the zygotes with purified FH2 domain, which perturbs the interaction of Spire2's KIND Domain with the FH2 domain of Formin2 , and thus perturbs actin nucleation in oocytes (Montaville et al., 2014). Next, we tracked the pronuclear migration of these zygotes, expressing H2B-mCherry and MyrGFP. These experiments were done together with Kathleen Scheffler, as the results were of interest to both of our experiments. The timing of these experiments was also complicated and better to achieve with two people. The results are shown in Figure 19.

Indeed, we found that in the presence of the purified $\mathrm{FH} 2$-His protein, the male pronucleus migrated slower away from the cortex than when MBP-His was injected, thus giving a similar result as Rab11a perturbation. This can be seen in the live imaging panels shown in Figure 19A, where the cyan box highlights the distance of the male pronucleus from the cortex 30 minutes after pronuclear formation. While in the MBP-His-injected zygote, the male pronucleus formed at the cortex and quickly migrated towards the centre, in the $\mathrm{FH} 2$-His-injected zygote, the male pronucleus formed at the cortex but did not migrate quickly towards the centre, rather growing in size still in close proximity to the cortex. As seen in later images, both the male and female pronuclei continued to migrate to the centre in both conditions until nuclear envelope breakdown. 
This migration is quantified in Figure 19B. In the zygotes with MBP-His, the male pronuclei formed with a mean distance of $36.52 \pm 2.31 \mu \mathrm{m}$ from the centre; after 15 minutes, they had migrated to $29.31 \pm 3.68 \mu \mathrm{m}$ from the centre, and by 30 minutes to $25.29 \pm 2.18 \mu \mathrm{m}$ from the centre. In the first 30 minutes, they migrated an average of $11.23 \mu \mathrm{m}$. In the presence of $\mathrm{FH} 2-\mathrm{His}$, the male pronuclei formed with a mean distance of $35.19 \pm 2.20 \mu \mathrm{m}$ from the centre of the zygote; after 15 minutes, they were still $32.63 \pm 1.30 \mu \mathrm{m}$ from the centre ( $p<0.001$ compared to wild-type, Welch's t-test), and even after 30 minutes they had not migrated past $30.88 \pm 1.20 \mu \mathrm{m}$ from the centre ( $p<0.001$ compared to wild-type, Welch's t-test), a mean migration of $4.31 \mu \mathrm{m}$ in 30 minutes. At the last time point before nuclear envelope breakdown, the male pronucleus was on average $10.41 \pm 1.40 \mu \mathrm{m}$ from the centre in MBP-His-injected cells, and $10.92 \pm 1.64 \mu \mathrm{m}$ in FH2-His-injected zygotes ( $p<0.001$, Welch's t-test). The female pronuclei shown on the right-hand-side graph formed closer to the centre than the male pronuclei, whether MBP-His or $\mathrm{FH} 2$-His was injected into the zygote. In the control group, the female pronuclei formed with a mean distance from the centre of $24.57 \pm 4.02 \mu \mathrm{m}$, and before nuclear envelope breakdown, had travelled to $11.41 \pm 1.76 \mu \mathrm{m}$ from the centre of the zygote. In the $\mathrm{FH} 2-\mathrm{His}$ group, the mean distance from the centre of the zygote at pronuclear formation was $23.53 \pm 2.16 \mu \mathrm{m}$, and $11.42 \pm 2.23 \mu \mathrm{m}$ at nuclear envelope breakdown, neither of which was significantly different from the control group.

Next, the average velocities in different time frames were calculated and are shown in Figure 19C. Several of the time frames show a significant difference in the velocity of migration for both the female and male pronuclei; generally, the initial faster migration of the first 30 minutes to one hour is slowed down in the presence of $\mathrm{FH} 2$, while later migration in the last hours before nuclear envelope breakdown are occasionally faster than in control cells. As for dominant-negative Rab11a-expressing zygotes in Figure 5, it is possible that the pronuclei travelling faster later may be to 'catch up' the fact they were slowed down initially, so our focus was the first phase of migration. In the first 30 minutes after pronuclear formation, the male pronucleus migrated at a mean velocity of $0.38 \pm 0.07 \mu \mathrm{m} /$ minute towards the centre in the MBP-His-injected group, and in the dominantnegative group the mean velocity was $0.15 \pm 0.05 \mu \mathrm{m} /$ minute $(p<0.001$, Welch's $t$-test); the female pronucleus moved at a mean velocity of $0.036 \pm 0.091 \mu \mathrm{m} /$ minute in the control cells, and $0.019 \pm 0.046 \mu \mathrm{m} / \mathrm{minute}$ in the dominant-negative group, even moving away from the cortex in this initial phase ( $p=0.0109$, Welch's t-test). While the male pronuclei did not show a significant change in velocity in the $0.5-1 \mathrm{~h}$ or $1-2 \mathrm{~h}$ post pronuclear formation time frames, the female pronuclei did show a significant decrease in velocity at the next two time points. Between $0.5-1 \mathrm{~h}$ after pronuclear migration, female pronuclei of MBP-His-injected zygotes moved at a mean velocity of $0.034 \pm 0.044 \mu \mathrm{m} /$ minute, compared to a mean velocity of $0.000 \pm 0.022 \mu \mathrm{m} /$ minute in the $\mathrm{FH} 2-\mathrm{His}$ 
group, essentially at a standstill. In the next time frame from $1-2 \mathrm{~h}$ post pronuclear formation, female pronuclei moved at a mean velocity of $0.027 \pm 0.026 \mu \mathrm{m} /$ minute towards the centre in control cells, compared to $0.011 \pm 0.023 \mu \mathrm{m} /$ minute in the $\mathrm{FH} 2$-His group.

Overall, these results indicate a role of Spire2-Formin-2 interaction in the early stages of pronuclear migration. In particular, the male pronucleus was unable to migrate as fast away from the cortex in the first 30 minutes when Spire2-Formin-2 interaction and thus actin nucleation was perturbed. Quantification indicated that migration of the female pronucleus was also affected during the first two hours of pronuclear migration, with female pronuclei on average showing an outward migration in the first 30 minutes, followed by barely any migration in the next half hour, and then slow migration inwards. Both male and female pronuclei were able to reach the centre of the zygote with no significant difference in the $\mathrm{FH} 2$-treated groups compared to the control. Indeed, both male and pronuclei migrated faster in the later stages of pronuclear migration in $\mathrm{FH} 2-\mathrm{His}$-injected zygotes compared to control cells. This is possibly due to the fact that the pronuclei still had more distance to cover than in MBP-His-injected zygotes.

With the knowledge that $\mathrm{FH} 2$ inhibits fast migration of pronuclei, my next goal was to investigate the effect of $\mathrm{FH} 2$ on the cortical enrichment we described for both mScarlet-Rab11a and mClover3-Spire2 at the forming male pronucleus. 
A

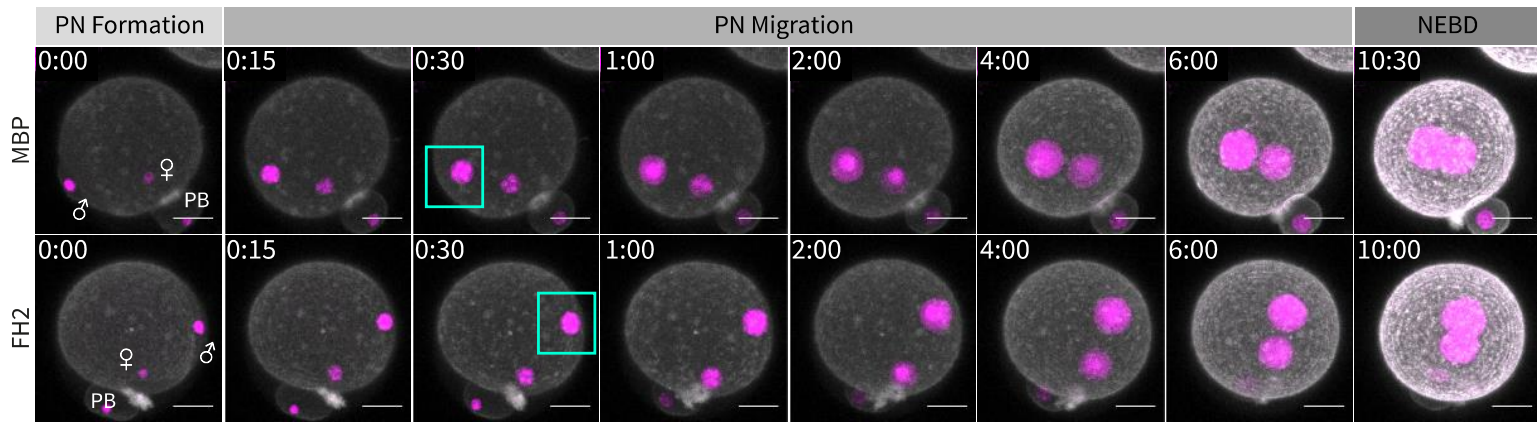

B

Migration of male pronucleus

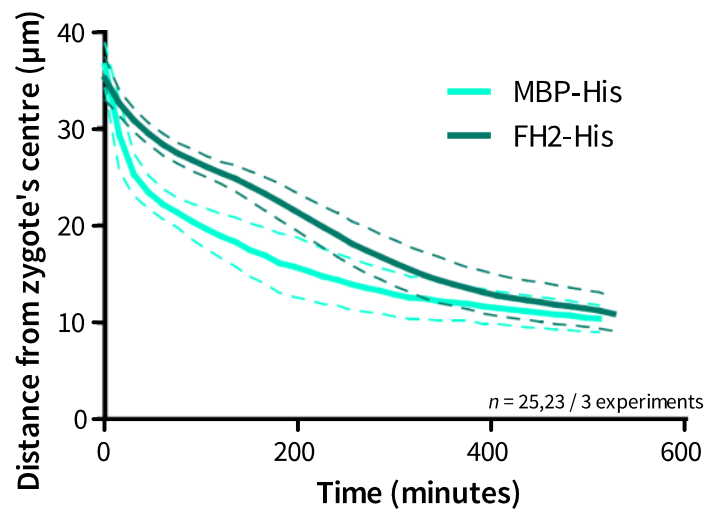

C

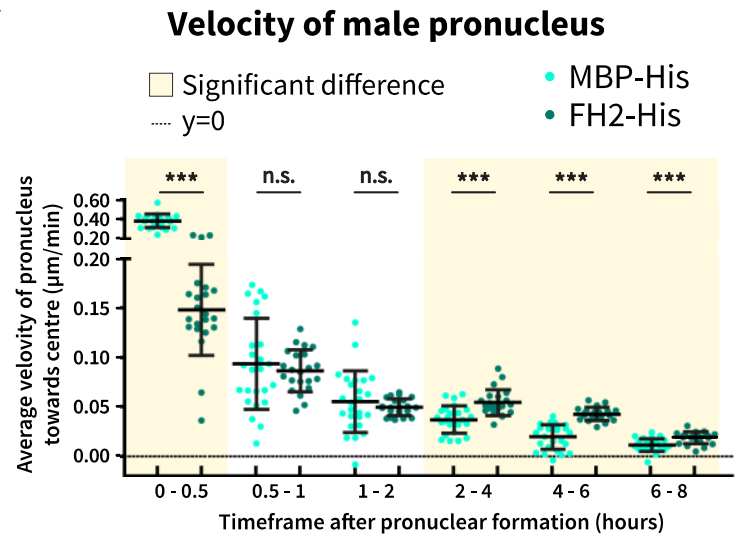

Migration of female pronucleus

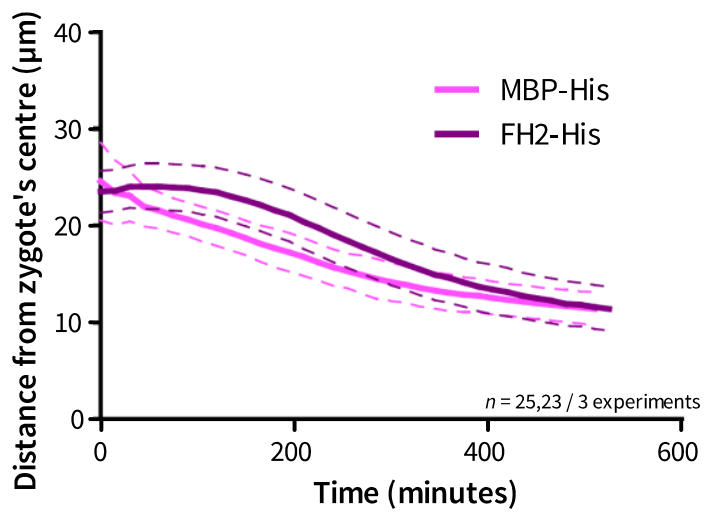

\section{Velocity of female pronucleus}

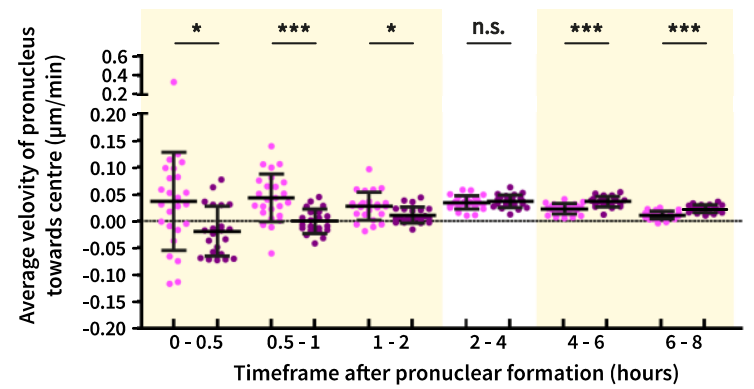

Figure 19: Spire2-Formin-2 interaction is required for fast migration of the male pronucleus. (A) Confocal images of live zygotes injected with either MBP-His or FH2-His purified protein, as well as MyrGFP (grey) and H2B-mCherry (magenta) mRNA. Cyan box highlights position of male pronucleus 30 minutes after pronuclear formation. Images taken every 15 minutes, every $3 \mu \mathrm{m}$ throughout the zygote. $P B=$ Polar body, $\sigma^{7}=$ male pronucleus $q=$ female pronucleus. Images shown are maximum intensity projections of the z-planes with a 1 px Gaussian blur filter applied. Images taken on Zeiss LSM-880 confocal microscope. Scale bar $=20 \mu \mathrm{m}$. Time shown in hours:minutes relative to pronuclear formation. Representative images; total $n=25$ for MBP-His, and $n=23$ for FH2-His from three experiments conducted together with Kathleen Scheffler. (B) Migration of female and male pronucleus over time. The live imaging data were reconstructed in 3D using IMARIS software, and the relative distance of each pronucleus from the centre of the zygote was calculated and plotted over time. On the left, migration of male pronucleus is shown, and on the right, the female, each comparing zygotes injected with MBPHis (light cyan and light magenta respectively), and FH2-His (dark cyan and dark purple respectively); dashed lines = one standard deviation. (C) Velocity of male (left) and female (right) pronucleus in different time frames. The mean velocity of each pronucleus for each time frame was calculated and plotted so that each dot represents one pronucleus; the mean of all pronuclei for each time frame was calculated and is indicated by the black line with error bars signifying one standard deviation. The colour key is identical to (B). Note the differing $y$-axes for male and female pronuclei, and that the dotted black line shows where $y=0$. Shaded yellow area signifies time frame in which there is a significant difference between the MBP-His and FH2-His groups. Statistical significance between conditions calculated with Unpaired, two-tailed t-test with Welch's correction. * $p \leq 0.05, * * p \leq 0.01, * * * p \leq 0.001$. 
mScarlet-Rab11a and mClover3-Spire2 accumulation by the male pronucleus require Spire2-Formin2 interaction

In the last section, we showed that interaction between Spire2 and Formin-2 was required for the first fast migration of pronuclei, indicating that actin nucleation by these two proteins is important for early migration. Earlier, we also described enrichment of mScarlet-Rab11a as well as mClover3-Spire2 adjacent to the fast-moving male pronucleus around the time of its formation, though we were not able to find out if they directly increase actin nucleation in this area. The next question was whether the interaction between Spire2 and Formin-2 was also required for this enrichment. For this, zygotes expressing either $m$ Scarlet-Rab11a (Figure 20A and B) or mClover3-Spire2 (Figure 20C and D) mRNA were imaged during formation of the male pronucleus, as previously described in Figures 13 and 17, in the presence of either MBP-His or FH2-His.

In Figure 20A, the results from the mScarlet-Rab11a imaging are shown. Zygotes expressing $m S c a r l e t-$ Rab11a (grey) and H2B-mEGFP (magenta) were injected with either MBP-His or FH2-His after fertilisation and subsequently imaged. An enrichment of mScarlet-Rab11a was observed adjacent to the forming male pronucleus, as indicated by the cyan arrow, in the zygote injected with MBP-His. It should be noted that an air bubble formed in the immersion oil of the objective around the time point of pronuclear formation, making the overall signal darker, though the enrichment was still visible. No such enrichment could be seen in the zygote injected with $\mathrm{FH} 2$-His, though vesicles were seen in the cytoplasm. The male pronucleus migrated slower away from the cortex compared to the male pronucleus shown above. This experiment was only repeated one time with mScarlet-Rab11a; as mentioned previously, we found that imaging with mClover3-Spire2 gave clearer results, as the enrichment was more obvious to distinguish. Therefore, I instead repeated this experiment three times with mClover3-Spire2 as shown below. However, this one experiment was still quantified, as shown in Figure 20B; 5/6 (83.333\%) of zygotes injected with MBP-His showed a Rab11a enrichment at formation, while $0 \%$ of $\mathrm{FH} 2$-His-injected zygotes showed this enrichment ( $p=0.0476$, two-tailed Fisher's exact test).

In Figure 20C, the results from the mClover3-Spire2 imaging are shown. Zygotes expressing $m$ Clover3Spire2 (grey) and H2B-mCherry (magenta) were injected with either MBP-His or FH2-His after fertilisation and imaged. In the MBP-His-injected zygotes, a clear enrichment was seen, with a weaker signal apparent at the fertilisation cone and a stronger signal at its edges at first, which became a bright accumulation as the male pronucleus formed, as indicated by the cyan arrow at 0:09. At the same time, the male pronucleus migrated quickly away from the cortex. In the FH2-His injected zygote, no such enrichment was detected, though mClover3-Spire2-positive vesicles were visible in the cytoplasm, as indicated by the cyan arrows. The male pronucleus formed but migrated slowly away 
from the cortex. This experiment was repeated three times, and quantifications are shown in Figure 20D; all zygotes injected with MBP-His showed an mClover3-Spire2 enrichment, compared to none of the FH2-His injected zygotes ( $p<0.001$, two-tailed Fisher's exact test).

In these experiments, it was shown that the enrichment of mScarlet-Rab11a and mClover3-Spire2 at the forming male pronucleus was dependent on the interaction between Spire2 and Formin-2. However, these results still did not allow us to distinguish between the role of cytoplasmic actin nucleation by these proteins, and the enrichment seen localised to the forming male pronucleus. Therefore, an overexpression approach was used next to further investigate the mechanisms at play. 

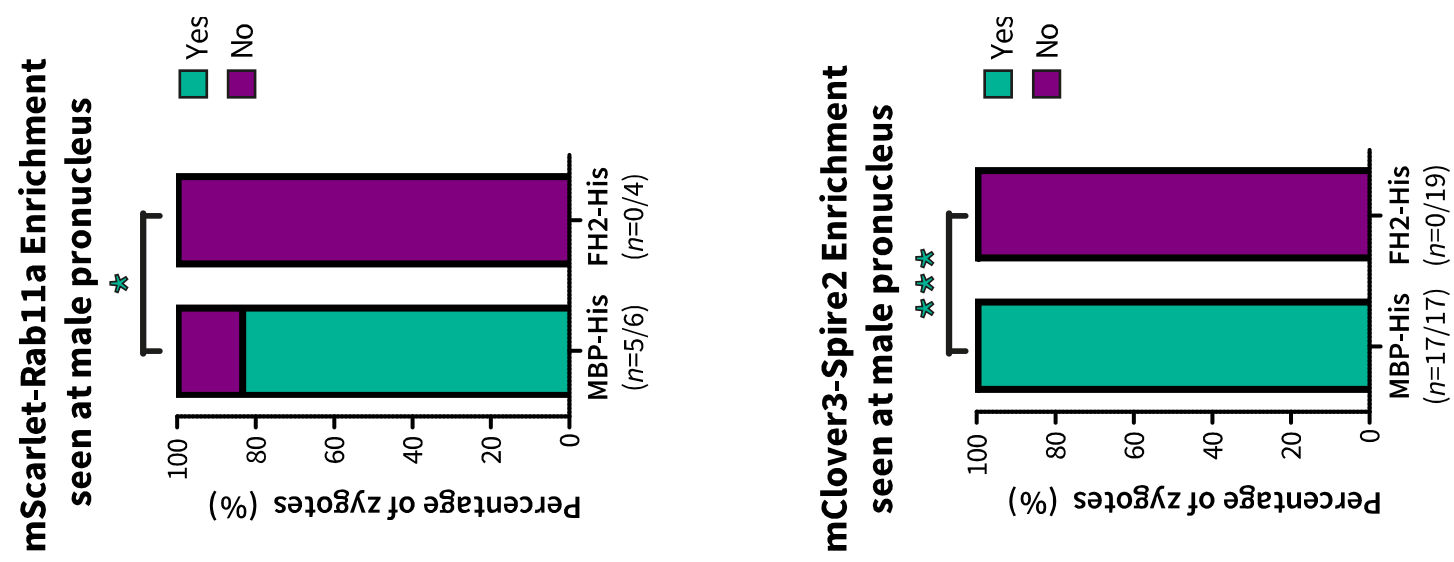

$m$
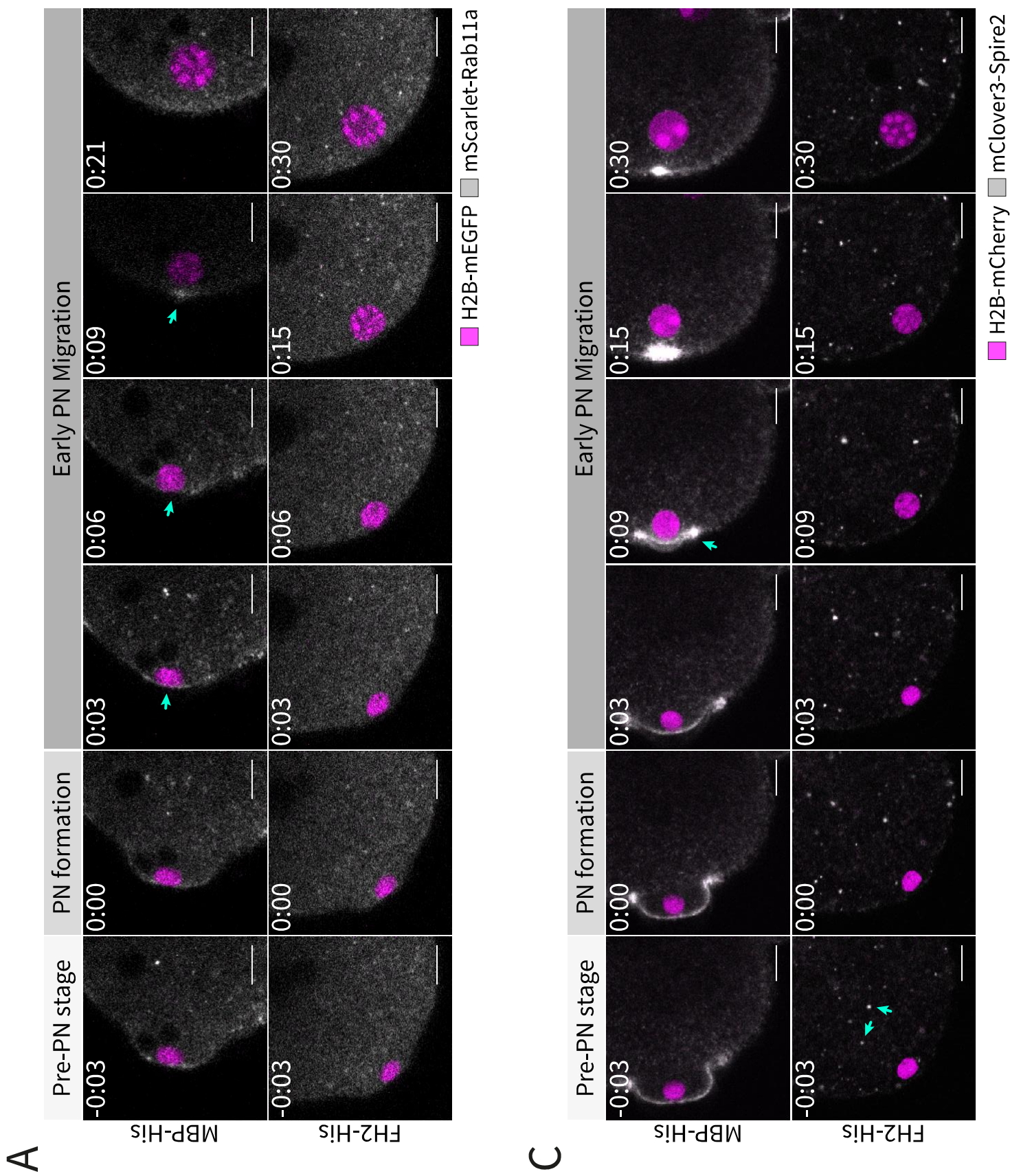
Figure 20: mScarlet-Rab11a and mClover3-Spire2 accumulation by the male pronucleus require Spire2-Formin-2 interaction. (A) Confocal images of male pronucleus forming close to the cortex of live zygote expressing mScarlet-Rab11a (grey) and H2B-mEGFP (magenta), injected with either MBP-His (top) or FH2-His (bottom), over time. Single z-planes of plane at which male DNA is central is shown. Cyan arrow indicates Rab11a accumulation as the pronucleus forms adjacent to the cortex; magenta arrow indicates cluster in cytoplasm. Images taken with a Zeiss LSM-800 microscope. Gaussian blur 1 px applied. Scale bar $=10 \mu \mathrm{m}$. Time shown in hours:minutes relative to pronuclear formation. Representative images from one experiment are shown, with $\mathrm{n}=9$ for $\mathrm{MBP}$-His, and $\mathrm{n}=9$ for $\mathrm{FH} 2$-His. (B) Quantification of enrichment seen at male pronuclei shown in (A). Presence of mScarlet-Rab11a enrichment was judged by eye, three separate times per zygote to avoid bias, with a yes/no read-out. Two-tailed Fisher's exact test used to test statistical significance; * $p \leq 0.05, * * p \leq 0.01, * * * p \leq 0.001$. (C) Confocal images of male pronucleus forming close to the cortex of live zygote expressing mClover3-Spire2 (grey) and H2BmEGFP (magenta), injected with either MBP-His (top) or FH2-His (bottom), over time. Single z-planes of plane at which male DNA is central is shown. Cyan arrow indicates Spire2 accumulation as the pronucleus forms adjacent to the cortex. Images taken with a Zeiss LSM-800 microscope. Gaussian blur 1 px applied. Scale bar $=10 \mu \mathrm{m}$. Time shown in hours:minutes relative to pronuclear formation. Representative images from three experiment are shown, with total $n=17$ for MBP-His, and $n=19$ for FH2-His. (D) Quantification of enrichment seen at male pronuclei shown in (C). Presence of mClover3-Spire2 enrichment was judged by eye, with a yes/no read-out. Two-tailed Fisher's exact test used to test statistical significance; * $p \leq 0.05, * *$ $p \leq 0.01, * * * p \leq 0.001$.

\section{Overexpression of Spire2 elongates fast migration of pronuclei}

To complement the observational and perturbational experiments shown, an overexpression assay was the next step. As described in Introduction section 1.5, Rab11a is small GTPase of the Rab family, specifically involved in the targeting of vesicles to the plasma membrane. As such, it is unlikely that overexpression of only the Rab11a protein would necessarily lead to an increase of Rab11a-positive vesicles or their function, as several additional points of regulation exist in this network (Stenmark, 2009). On the other hand, both Spire2 and Formin-2 overexpression with mRNA have been used previously in mouse oocytes to induce an increase in actin nucleation (Pfender et al., 2011). As Formin2-EGFP shown in Figures 15 and 29 was not visible without Spire2 expression, I thus chose to try Spire2 overexpression in zygotes to see its effect on pronuclear migration. Note that this experiment was done prior to optimisation of mClover3-Spire2, and therefore mEGFP-Spire2 was still used.

For this experiment, mEGFP-Spire2 was overexpressed at two different concentrations of mRNA: $1200 \mathrm{ng} / \mu \mathrm{L}$, and $400 \mathrm{ng} / \mu \mathrm{L}$, together with $H 2 B$-mCherry, and the whole zygotes were imaged every 15 minutes. The results of this are shown in Figure 21. In the first example, of a total of 7 zygotes imaged in this high concentration group, a very large ring of localisation around the forming male pronucleus was seen, indicated by the cyan arrows at $-0: 15$ and 0:00. This had a similar but much larger localisation compared to the pattern seen with imaging of mClover3-Spire2 shown in Figure 17. As the male pronucleus formed, this localisation of mEGFP-Spire2 became more focused above it, as indicated by the cyan arrow at 0:15. In the next 15 minutes, both male and female pronuclei migrated very quickly to the side opposite to the mEGFP-Spire2 localisation, moving all the way to the opposite cortex by 0:30, indicated by the cyan box. While in lower concentrations of Spire2 imaging done previously, this bright spot-like localisation would fade quickly, here it remained even until four hours after pronuclear formation. In addition to the mEGFP-Spire2 localisation seen near the male 
pronucleus, other areas of the cortex also showed bright spots, indicated by the magenta arrows over time. The large area of mEGFP-Spire2 localisation, near the polar body at 1:30, faded over time, with the signal becoming more heterogenous in the cytoplasm. The nuclei began to migrate to the centre as the localised signal faded; however, in this example, mEGFP-Spire2 was later seen as a bright area of spots on one side of the zygote, as indicated by the cyan arrows, with the pronuclei still somewhat on the opposite side.

In the zygote shown in the second panel of Figure 21, the same high level of overexpression of $m E G F P$ Spire2 mRNA also resulted in elongated, fast pronuclear migration, though the extent of this was lower. Again, a ring-like localisation of mEGFP-Spire2 was seen at the cortex, as indicated by the cyan arrows, with quite a distance to the fertilisation cone, and this ring concentrated over time to form a smaller accumulation over the male pronucleus. As in the first example, both the male and female pronuclei migrated quickly over the next hour, not quite reaching the opposite cortex, as indicated by the cyan box. The mEGFP-Spire2 enrichment did not completely fade until over four hours after pronuclear formation. Bright spots of mEGFP-Spire2 were also seen at other areas of the cortex in the first hours of imaging, indicated by the magenta arrows. However, unlike the first example, no bright cortical localisation returned after this point, and the pronuclei migrated to the centre, where they remained until the end of imaging.

In the two lower panels of Figure 21, two examples of zygotes expressing a lower (though still high) concentration of mEGFP-Spire 2 mRNA are shown. In the first example, accumulation of mEGFP-Spire2 appeared around the fertilisation cone, but not as far away from the male pronucleus as in the higher concentrations described above. This constricted to formed a bright spot, which was present until over 3 hours after pronuclear formation. As opposed to the fast migration of both pronuclei to the opposite side of the cortex, the female pronucleus did not appear to be affected by the Spire2 overexpression, while the male pronucleus appeared to move somewhat faster than normal, migrating slightly past the centre of the zygote as indicated by the cyan box. The pronuclei were able to migrate to the centre later, where they stayed until the end of imaging. In this zygote, further bright spots of mEGFP-Spire2 were seen in different cortical regions as indicated by the magenta arrows.

In the second example, from a total of 9 zygotes imaged in this group, the mEGFP-Spire2 enrichment seen was only small by the male pronucleus, as indicated by the cyan arrow, and disappeared around 2:15 post pronuclear formation. The male pronucleus formed and migrates past the centre, as indicated by the cyan boxes. In this example, the pronuclei eventually migrated to the centre, though they appeared to take longer than in previous examples. No additional spots of mEGFP-Spire2 enrichment were observed in this zygote. 
In this experiment, it was found that higher concentrations of mEGFP-Spire2 mRNA expressed in zygotes was sufficient to induce fast migration of both pronuclei away from the localisation of mEGFPSpire2. Furthermore, the localisation of mEGFP-Spire2 was found to form a bright ring quite far away from the fertilisation cone, which became smaller and localised over the male pronucleus as it formed; also, mEGFP-Spire2 signal could be observed at other spots throughout the cortex. At slightly lower concentrations, the male pronucleus would migrate slightly too far in the first hours after pronuclear formation, but the female pronucleus did not appear to be affected. One additional observation made here was that even 15 hours post pronuclear formation, when pronuclei had long reached the centre, none of the zygotes imaged (total of 16 from the two groups) were able to undergo the first mitotic division, likely as Spire2 is also involved in cytokinesis. As this experiment was a preliminary test of the effect of overexpression of Spire2, it was not repeated again; rather, Kathleen Scheffler used these observations and eventually quantified pronuclear migration in response to overexpression of SNAPSpire2 mRNA, as shown in figure 30 in the appendix.

As this experiment provided evidence that Spire2 overexpression could speed up pronuclear migration of both male and female pronuclei, we next investigated whether the mScarlet-Rab11a and mClover3Spire2 enrichments could also be observed if the female pronucleus formed close to the cortex. 


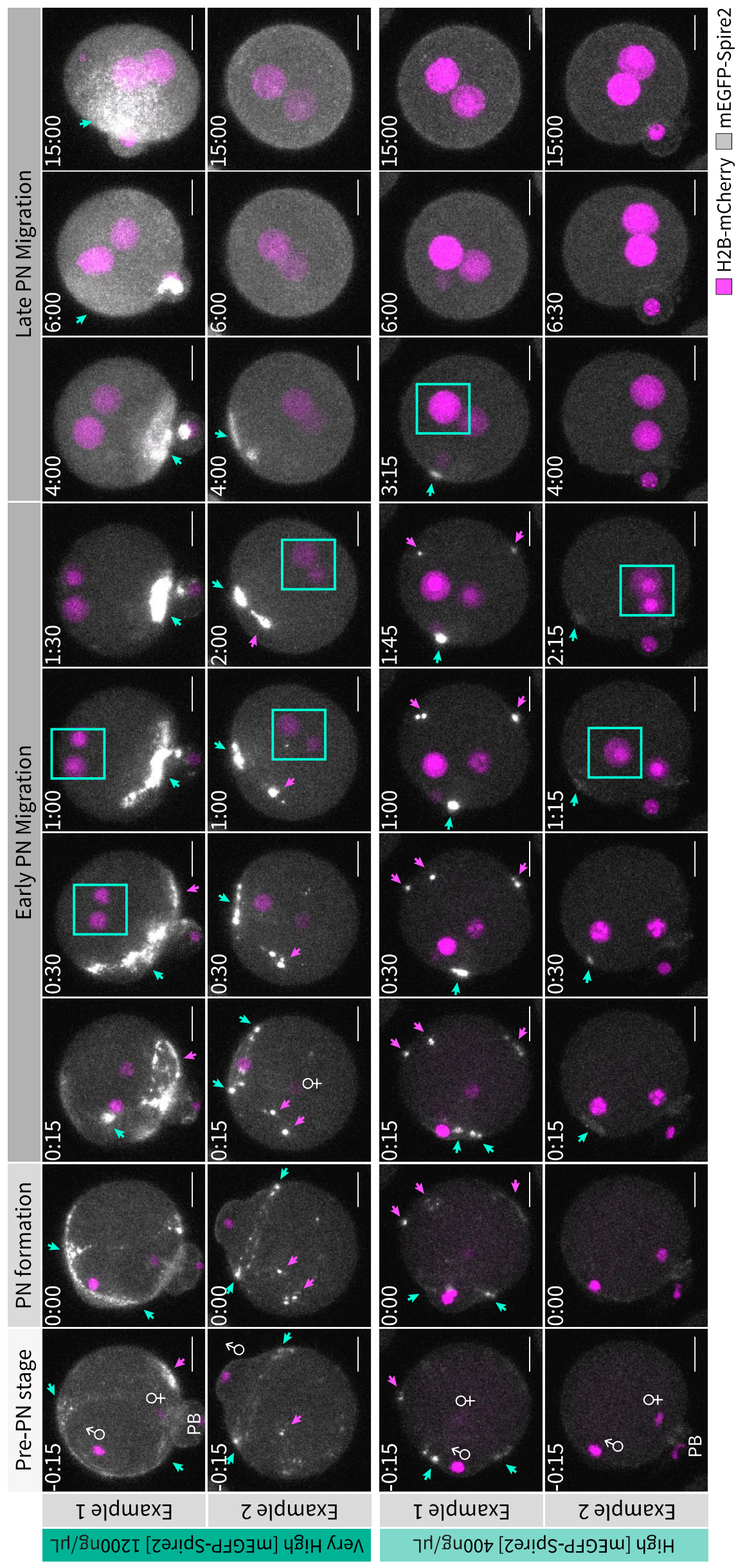


Figure 21: Overexpression of mEGFP-Spire2 elongates fast migration of pronuclei. Confocal images of zygotes expressing different concentrations of mEGFP-Spire2 (grey) and H2B-mCherry mRNA (magenta), over time. Zygotes were imaged every 15 minutes, with a z-resolution of $3 \mu \mathrm{m}$. $z$-projections of $28 \times 3 \mu \mathrm{m}$ are shown with Gaussian blur of $1 \mathrm{px}$. PB= Polar body, $\sigma^{7}=$ male pronucleus $q=$ female pronucleus. Cyan arrows indicate mEGFP-Spire 2 accumulation around forming male pronucleus, magenta arrows indicate other localisations of MEGFP-Spire2. Cyan box indicates position of pronuclei. Images taken with a Zeiss LSM-880 microscope. Gaussian blur 1 px applied. Scale bar $=20 \mu \mathrm{m}$. Time shown in hours:minutes relative to pronuclear formation. Images from two representative zygotes are shown per group, from one experiment; $\mathrm{n}=7$ zygotes for the $1200 \mathrm{ng} / \mathrm{\mu L}$ group, and $\mathrm{n}=6$ for the $400 \mathrm{ng} / \mu \mathrm{L}$ dilution group. 
3.6 Fast migration of pronuclei and enrichment of Spire2, Rab11a are not dependent on sperm entry site, nor the fertilisation cone

The female pronucleus exhibits fast migration and mScarlet-Rab11a, mClover3-Spire2 accumulation when it forms close to the cortex

One key question remaining was whether the very fast migration observed in the first 30 minutes of male pronuclear migration was inherent to the site of sperm entry and the fertilisation cone, or other mechanisms and signalling pathways were involved, not inherent to the male DNA. As explained in the Introduction section 1.4, Kathleen Scheffler made the observation that as a side effect of nocodazole treatment, female pronuclei would often form closer to the cortex, and show a fast inward migration, similar to the male pronuclei. In the next set of experiments, mScarlet-Rab11a and mClover3-Spire2 were imaged in zygotes treated with DMSO or nocodazole, and the formation and migration of female pronuclei were imaged. The results of these experiments are shown in Figure 22.

First, I imaged the localisation of mScarlet-Rab11a in zygotes treated with either DMSO or nocodazole. The example images shown In Figure 22A are from a female pronucleus forming deeply in a DMSOtreated zygote, and a female pronucleus forming peripherally in a nocodazole-treated zygote. When the female pronucleus formed deep in the cell, no mScarlet-Rab11a enrichment was observed. However, in the lower panels, the maternal DNA was still positioned deep in the cell 45 minutes prior to pronuclear formation, but localised to the cortex prior to pronuclear formation. As the pronucleus formed peripherally, mScarlet-Rab11a localised to the cortical area adjacent to it, as indicated by the cyan arrows. It appeared as though a small 'cone' or protrusion formed around the maternal DNA at the cortex, similar to the fertilisation cone at the site of sperm entry. The female pronucleus could then be seen to migrate quickly away from the cortex. It should be noted that most, but not all female pronuclei formed peripherally (at the cortex) in the nocodazole group (18/31), and a few female pronuclei unexpectedly formed at the at the cortex in the DMSO group $(5 / 21)(p=0.0227$, two-tailed Fisher's exact test); the quantification of this are shown in Figure 22B. Thus, to quantify enrichment based on proximity to cortex, both DMSO and nocodazole groups were first sorted by whether the pronucleus formed close to the cortex or not; then, whether or not mScarlet-Rab11a was enriched at the cortex was scored for each zygote. The results of this quantification from four experiments are seen in Figure $22 \mathrm{C}$ and $\mathbf{D}$. In the DMSO group, the majority of cells formed deep inside the cell, and $0 / 16$ of these showed mScarlet-Rab11a enrichment. As mentioned, in five zygotes of the DMSO group, the female pronucleus also formed at the cortex. This was unexpected, but may have been due to slight differences in experimental set-up (see Methods section 2.2) and delay in putting zygotes on the microscope; perhaps they were not kept warm enough, and the cold-sensitive microtubules fell apart, creating the same effect as nocodazole. Regardless of the reason for this difference, it was 
found that in the DMSO group, $5 / 5$ of the zygotes in which the female pronuclei formed peripherally showed mScarlet-Rab11a enrichment ( $p<0.001$ compared to those forming deeply, two-tailed Fisher's exact test). In the nocodazole group, 13 zygotes did not have peripherally forming female pronuclei, which also did not show mScarlet-Rab11a enrichment. However, 17 of 18 of the zygotes in which female pronuclei formed at the cortex in response to nocodazole treatment showed mScarlet-Rab11a accumulation adjacent to the female pronucleus ( $p<0.001$ compared to those forming deeply, twotailed Fisher's exact test).

Next, mClover3-Spire2 was imaged in the same way as mScarlet-Rab11a above, to confirm that it too localised adjacent to the female pronucleus when it formed at the cortex. Note that of four experimental repetitions, one was conducted by me, and three by Kathleen Scheffler; images shown are from my repetition, and quantifications from all experiments pooled together. In the images shown in Figure 22E, the top panels show a female pronucleus forming inside a DMSO-treated zygote. While mClover3-Spire2 was seen at the site of polar body extrusion, no enrichment was seen as the pronucleus formed. In the nocodazole-treated zygote, the maternal DNA was initially inside the cell, as shown 54 minutes prior to pronuclear formation. At this time point, we could also see a side-effect of the nocodazole treatment, as chromosomes began to fall apart slightly, indicated by the magenta arrow. By the time the pronucleus formed, the chromosomes were at the cortex, and a clear cone had formed around them, with mClover3-Spire2 enrichment seen at its periphery, indicated by the cyan arrows. As the pronucleus formed and migrated quickly away from the cortex, the cone constricted, and mClover3-Spire2 enrichment could be seen overlying the female pronucleus in a bright accumulation. In these experiment, extra care was given to avoid DMSO-treated cells also having perturbed microtubules by ensuring they were kept warm at all times, and as can be seen in Figure 22F, only nocodazole-treated zygotes showed peripherally-forming female pronuclei in the four experiments $(p<0.001$, two-tailed Fisher's exact test). The quantifications for DMSO- and nocodazole-treated groups are shown in Figure $\mathbf{2 2} \mathbf{G}$ and $\mathbf{H}$ respectively. While there were no peripherally-forming female pronuclei in the DMSO group, one of the deep forming female pronuclei did show some mClover3-Spire2 enrichment at the cortex; it was not as strong as shown above, but a slight 'ring' of signal could be observed, and it was thus counted as such. However, the majority $(20 / 21)$ did not show any enrichment of mClover3-Spire2 when they formed deep in the cell. In the nocodazole-treated group, none (0/5) of the deep-forming pronuclei were accompanied by any mClover3-Spire2 enrichment, while 100\% (28/28) of peripherally-forming pronuclei showed the same pattern of $m$ Clover3-Spire2 enrichment as shown in the example in Figure $22 E$ ( $p<0.001$, two-tailed Fisher's exact test). 
In these experiments, we were able to find that the mScarlet-Rab11a and mClover3-Spire2 enrichment described at the site of pronuclear formation was not unique to male pronuclei, but indeed could be seen adjacent to female pronuclei when they formed close to the cortex. We continued to investigate the nature of this fast migration in the next set of experiments, which perturbed the actomyosin contractions of the fertilisation cone. 
A

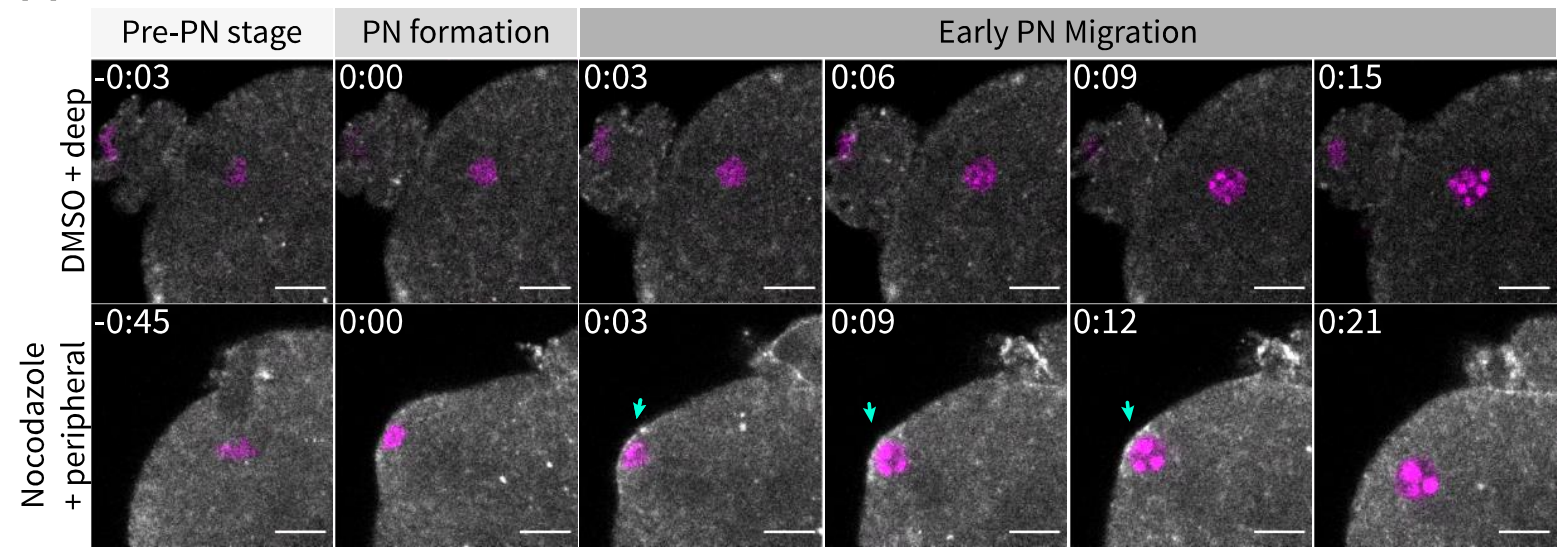

$\square$ H2B-mEGFP $\square$ mScarlet-Rab11a

B

Location of female pronucleus at formation

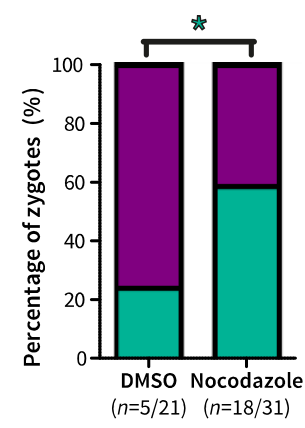

C

mScarlet-Rab11a Enrichment seen at female pronucleus: DMSO group

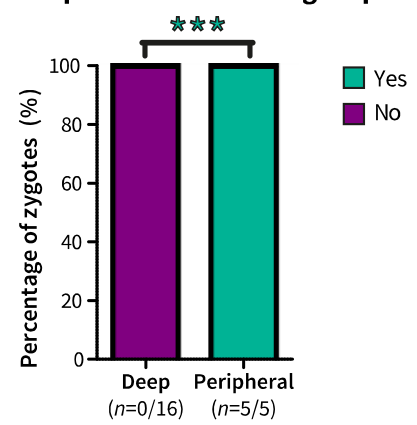

D

mScarlet-Rab11a Enrichment seen at female pronucleus: Nocodazole group

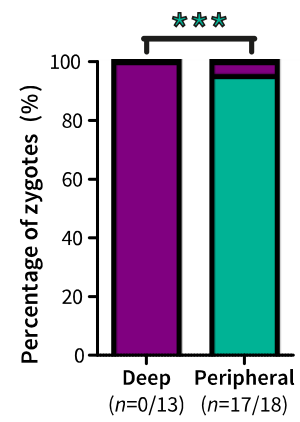

$\mathrm{E}$

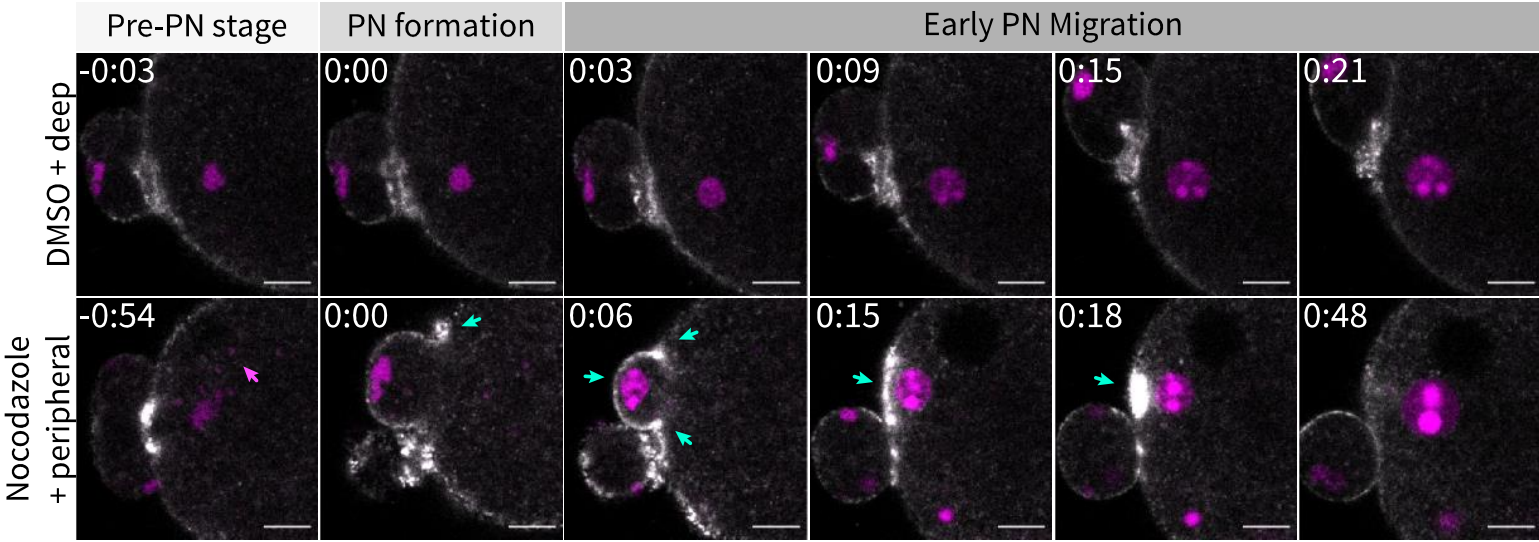

$\square$ H2B-mCherry $\square$ mClover3-Spire2

F

Location of female pronucleus at formation

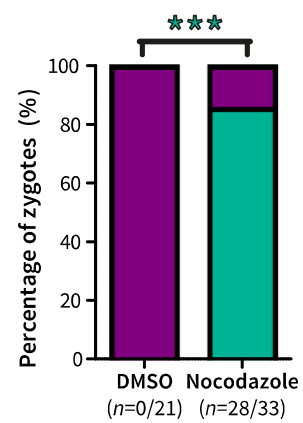

G

mClover3-Spire2 Enrichment seen at female pronucleus: DMSO group

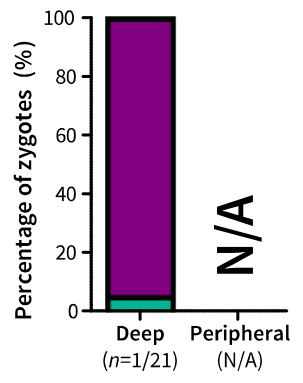

Page 118 of 177
$\mathrm{H}$

mClover3-Spire2 Enrichment seen at female pronucleus: Nocodazole group

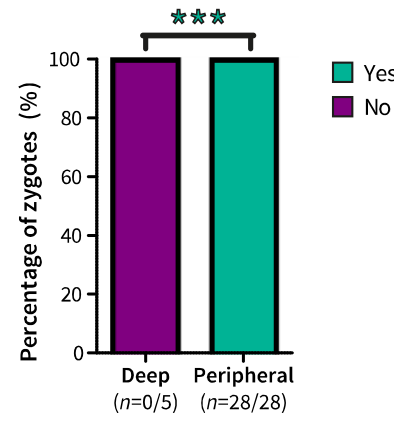


Figure 22: The female pronucleus exhibits fast migration and mScarlet-Rab11a, mClover3-Spire2 accumulation when it forms close to the cortex. (A) Confocal images of forming female pronucleus of live zygote expressing mScarlet-Rab11a (grey) and H2B-mEGFP (magenta), incubated with either DMSO and with deep pronuclear formation (top) or with nocodazole inducing peripheral pronuclear formation (bottom), over time. Single z-planes of plane at which female DNA is central is shown. Cyan arrow indicates Rab11a accumulation as the pronucleus forms adjacent to the cortex. Images taken with a Zeiss LSM-800 microscope. Gaussian blur 1 px applied. Scale bar $=10 \mu \mathrm{m}$. Time shown in hours:minutes relative to pronuclear formation. Representative images from four experiments are shown, with $n=21$ for DMSO, and $n=31$ for nocodazole. (B) Quantification of location of female pronuclear formation in DMSO and nocodazole treated zygotes. Two-tailed Fisher's exact test used to test statistical significance; * $p \leq 0.05, * * p \leq 0.01, * * * p \leq 0.001$. (C and D) Quantification of mScarlet-Rab11a enrichment at female pronuclei in DMSO-treated and nocodazole treated zygotes respectively. Female pronuclei formed either deep in the cell or peripherally at the cortex, and either showed enrichment or did not. Presence of mScarlet-Rab11a enrichment was judged by eye, three separate times per zygote to avoid bias, with a yes/no read-out. Two-tailed Fisher's exact test used to test statistical significance; * $p \leq 0.05, * * p \leq 0.01, * * * p \leq 0.001$. (E) Confocal images of forming female pronucleus of live zygote expressing mClover3-Spire2 (grey) and H2B-mEGFP (magenta), incubated with either DMSO and with deep pronuclear formation (top) or with nocodazole inducing peripheral pronuclear formation (bottom), over time. Single z-planes of plane at which male DNA is central is shown. Cyan arrow indicates Spire2 accumulation as the pronucleus forms adjacent to the cortex; magenta arrow indicates chromosomes falling apart in nocodazole-treated cell. Images taken with a Zeiss LSM-800 microscope. Gaussian blur 1 px applied. Scale bar $=10 \mu \mathrm{m}$. Time shown in hours:minutes relative to pronuclear formation. Representative images from four experiment are shown, with total $n=21$ for DMSO, and $n=33$ for nocodazole. Note that one of four experiments for mClover3-Spire2 was done by me, with the remaining done by Kathleen Scheffler; images are shown from my repetition. (F) Quantification of location of female pronuclear formation in DMSO and nocodazole treated zygotes. Two-tailed Fisher's exact test used to test statistical significance; * $p \leq 0.05,{ }^{* *} p \leq 0.01,{ }^{* * *}$ $p \leq 0.001$. (G and $H$ ) Quantification of mScarlet-Rab11a enrichment at female pronuclei in DMSO-treated and nocodazole treated zygotes respectively. Female pronuclei formed either deep in the cell or peripherally at the cortex, and either showed enrichment or did not; note that no female pronuclei in DMSO-treated cells formed peripherally in these experiments. Presence of mClover3-Spire2 enrichment was judged by eye, with a yes/no read-out. Two-tailed Fisher's exact test used to test statistical significance; * $p \leq 0.05, * * p \leq 0.01, * * * p \leq 0.001$.

\section{Fast pronuclear migration is possible without actomyosin contractions of the fertilisation cone}

In mouse zygotes, the fertilisation cone has been described to show rhythmic actomyosin contractions prior to pronuclear formation, which could be perturbed by MLCK-inhibitor ML-7 (Ajduk et al., 2011). ML-7 selectively inhibits the catalytic nature of MLCK (Saitoh et al., 1987), thus perturbing function of MLCK-dependent myosins, including myosin-II that is active in actomyosin contractions of the fertilisation cone. As it was possible that these actomyosin contractions were involved in the fast migration of pronuclei away from the cortex, I next tracked pronuclear migration in zygotes treated with either DMSO or ML-7. The exact protocol can be found in Methods section 2.2. Note that as opposed to other drug treatment experiments, ML-7 required two changes that worsened overall culture conditions: First, ML-7-containing medium could not be covered with paraffin oil, meaning that evaporation was more likely; and second, to reach a final ML-7 concentration of $30 \mu \mathrm{M}$, a dilution factor of drug and DMSO of 1:333 had to be used, as opposed to 1:1000 for other drug experiments. Furthermore, ML-7 affects not only actomyosin contractions of the fertilisation cone, but has also been shown to alter other myosin-related functions such as cortical tension in oocytes (Larson et al., 2010), and extrusion of the second polar body in zygotes (Matson et al., 2006), and thus may be involved in a number of processes of the zygote.

First, the conditions for ML-7 incubation were optimised. In the literature, ML-7 had been dissolved in either DMSO, or ethanol and water. When this experiment was attempted with ML-7 dissolved in 
ethanol and water, the zygotes developed very poorly, and often showed severe blebbing (data not shown). For this reason, the ML-7 powder was instead dissolved in DMSO, and this was used for the three experiments tracking pronuclear migration shown in Figure 23. While these cells still did not develop optimally as shown in the images, it was significantly better than in the experiments using ethanol and water. To ensure that ML-7 was indeed active during the duration of imaging, immature GV oocytes were placed in the imaging dish with the zygotes; ML-7 disrupts polar body extrusion in mouse oocytes (Matson et al., 2006), and rate of polar body extrusion was counted after imaging of zygotes was completed. For the three experiments conducted, a total of $36 \mathrm{GV}$ oocytes were placed with the DMSO groups, $78 \%$ of which had polar body extrusion; 37 GV oocytes were placed with the ML-7 groups, none of which had polar body extrusion ( $p<0.001$, two-tailed Fisher's exact test). Furthermore, even though zygotes in the ML-7 group showed nuclear envelope breakdown towards the end of imaging, cleavage of the first mitotic division failed, indicating that ML-7 was functional and inhibiting MLCK activity until the end of imaging.

Zygotes expressing MyrGFP and H2B-mCherry mRNA were imaged to allow pronuclear migration. The images can be seen in Figure 23A; one example of a DMSO zygote is shown, and two zygotes from the ML-7 group are shown. In the DMSO group, both pronuclei formed as normal, and the male pronucleus could be seen migrating away from the cortex quickly in the first 30 minutes after pronuclear migration, as indicated by the cyan box. In the later development of the zygote, the pronuclei did not appear to migrate as well into the centre as shown for previous control groups in Figures 5 and 19, possibly due to the higher concentration of DMSO and poor culture conditions required for this experiment. Regardless, by nuclear envelope breakdown, the pronuclei were at the centre, and mitotic division occurred successfully.

Two ML-7 groups are shown, as two different scenarios were seen in ML-7 treated zygotes. As mentioned previously, ML-7 inhibits cytokinesis of polar body extrusion, and thus, if it was added prior to the polar body having formed, both sets of maternal DNAs would remain in the zygote. This case is shown in the first example. Note that for quantification of pronuclear migration, both female pronuclei were tracked in these cases. Interestingly, these sets of maternal DNAs would often form pronuclei at the cortex as a result. In turn, all three forming pronuclei of this zygote showed a fast inwards migration towards the centre of the zygote, indicating that the fast migration of pronuclei was possible in the absence of MLCK activity and actomyosin contractions. In other zygotes, the polar body had already formed, and one of these examples is shown as the second ML-7-treated zygote. As can be seen, the polar body was present, and the female pronucleus formed in its usual place deeper inside the cell. The male pronucleus formed at the cortex, and migrated quickly towards the centre, as indicated by the cyan box. Interestingly, the MyrGFP signal shows an accumulation by the male 
pronucleus, though it is not clear why. While in some zygotes, the polar body stayed intact throughout pronuclear migration, in other zygotes, including this example, the polar body later collapsed; here, this is shown between time points 8:00 and 8:15, when the polar body collapsed and its DNA entered the zygote. Note that these pronuclei appearing later were not quantified for tracking of pronuclear migration. Neither the first nor second ML-7-treated zygote appeared to be developing completely normally, as the pronuclei did not migrate completely to the centre. Also, cortical spots could be seen as MyrGFP expression increased over time, possibly indicating cortical disruptions. As mentioned, nuclear envelope breakdown occurred, but ML-7 treated zygotes were not able to undergo cytokinesis.

This migration is quantified in Figure 23B. Overall, for the migration of the male pronuclei, it appears that the migration patterns of both DMSO and ML-7 groups overlapped fairly well; both showed an early, fast migration towards the centre. In the DMSO-treated zygotes, the male pronuclei formed with a mean distance of $36.14 \pm 2.51 \mu \mathrm{m}$ from the centre; by 30 minutes, they had migrated to $24.70 \pm 1.69 \mu \mathrm{m}$ from the zygote's centre. Thus, in the first 30 minutes, they migrated an average of $11.44 \mu \mathrm{m}$. In the presence of ML-7, the male pronuclei formed with a mean distance of $34.63 \pm 3.89 \mu \mathrm{m}$ from the centre of the zygote, and after 30 minutes, they had migrated to $26.16 \pm 4.45 \mu \mathrm{m}$ from the centre ( $p>0.05$, Welch's test), a mean migration of $8.47 \mu \mathrm{m}$ in 30 minutes. Later, both groups showed a plateau of inward migration, lasting approximately 90 minutes, not usually seen in control cells shown previously. It is possible that this was due to the culture conditions mentioned. Ninety minutes after pronuclear formation, the male pronucleus was on average $20.62 \pm 1.76 \mu \mathrm{m}$ from the centre in DMSO-treated cells, and $21.23 \pm 1.85 \mu \mathrm{m}$ in ML-7 treated zygotes $(p>0.05$, Welch's t-test), and they both did not migrate far the next 90 minutes. However, they did continue to migrate further later on, as by the last time point before nuclear envelope breakdown, the pronuclei of both groups were at similar distances from the centre; in the DMSO group, the mean distance from the centre was $12.14 \pm 2.21 \mu \mathrm{m}$, and in the ML-7 group, it was $11.22 \pm 3.01 \mu \mathrm{m}(p>0.05$, Welch's t-test).

The female pronuclei are shown on the right-hand side graph. As shown in the images of Figure 23A, some female pronuclei formed close to the cortex in ML-7 treated zygotes, but not in DMSO-treated zygotes. In the DMSO group, the female pronuclei formed with a mean distance from the centre of $23.81 \pm 2.69 \mu \mathrm{m}$, compared to $32.39 \pm 7.12 \mu \mathrm{m}$ in the ML-7 group ( $<<0.001$, Welch's t-test), a value much closer to the male pronuclei at this time point. Within the first 30 minutes, the female pronuclei in the ML-7 group had migrated to $24.18 \pm 4.55 \mu \mathrm{m}$, compared to $22.51 \pm 2.38 \mu \mathrm{m}$ in the DMSO group, which still had a significant difference ( $p=0.03$, Welch's t-test). Like the male pronuclei described above, the female pronuclei did not migrate further inward after this fast migration, in fact moving 
slightly outward before continuing migrating towards the centre, which resulted in them not reaching as close to the centre as in DMSO-treated cells. Before nuclear envelope breakdown, female pronuclei in DMSO-treated zygotes had travelled to $13.17 \pm 1.72 \mu \mathrm{m}$ from the centre, and $16.90 \pm 2.34 \mu \mathrm{m}$ in the ML-7 treated group ( $p<0.01$, Welch's t-test).

To further analyse these data, the average velocities in different time frames were calculated and are shown in Figure 23C. The velocities in male pronuclei were significantly different in first and second half hours, but not later time frames. In the first 30 minutes, the male pronucleus moved at a mean velocity of $0.39 \pm 0.05 \mu \mathrm{m} /$ minute towards the centre in the DMSO group, and $0.31 \pm 0.14 \mu \mathrm{m} / \mathrm{minute}$ in the ML-7 group ( $p=0.0068$, Welch's t-test). In the next time frame from 0.5 - 1 hour after pronuclear formation, the DMSO group had an average velocity of $0.085 \pm 0.034 \mu \mathrm{m} /$ minute towards the centre, compared to $0.14 \pm 0.13 \mu \mathrm{m} /$ minute in the ML-7 group ( $p=0.0349$, Welch's t-test). Thus, while ML-7 caused a slower migration of the male pronucleus in the first 30 minutes compared to DMSO, the next 30 minutes were faster in the ML-7 group than in the DMSO group. Overall, it appeared that there was simply a much higher variability to the velocities in the first hour, but male pronuclei migrated as far into the centre within the first hour in ML-7 and DMSO groups. The effect described here was not similar to the effect of Rab11a or actin nucleation perturbations shown in Figure 5 and 19 respectively; many male pronuclei were still able to migrate quickly away from the cortex, even with perturbed MLCK activity, indicating that it was not required for the fast migration, even if it did clearly change some dynamics in the zygote.

When looking at the velocities of female pronuclei, it is further clear that MLCK was not required for the fast migration of pronuclei; in fact, the female pronuclei migrated with an increased velocity only in the ML-7 group, as some of them formed close to the cortex. The female pronuclei moved at a mean velocity of $0.038 \pm 0.060 \mu \mathrm{m} /$ minute in the DMSO group, compared to $0.29 \pm 0.17 \mu \mathrm{m} /$ minute in the ML-7 group ( $p<0.001$, Welch's t-test). The high variability in the ML-7 group can be explained by the fact that some female pronuclei still formed at their usual position inside the cell, thus migrating slowly towards the centre. In the next time frame, the female pronuclei were still faster in the ML-7 group than the DMSO group; in the DMSO group, the mean velocity of female pronuclei was $0.038 \pm 0.048 \mu \mathrm{m} /$ minute, and $0.10 \pm 0.09 \mu \mathrm{m} /$ minute in the ML-7 group ( $p<0.001$, Welch's t-test). The remaining time frames did not show a significant difference between the groups, neither for the male nor female pronuclei.

While these results did show that the culture conditions in ML-7 experiments were likely subpar, and that MLCK is likely involved in many processes in the early zygote, it does not appear that it was absolutely essential for the fast migration of pronuclei that formed close to the cortex. As we had 
observed mClover3-Spire2 localisation to the fertilisation cone, which could be linked to the actomyosin contractions, the next experiments looked at mClover3-Spire2 localisation in zygotes treated with ML-7.

A
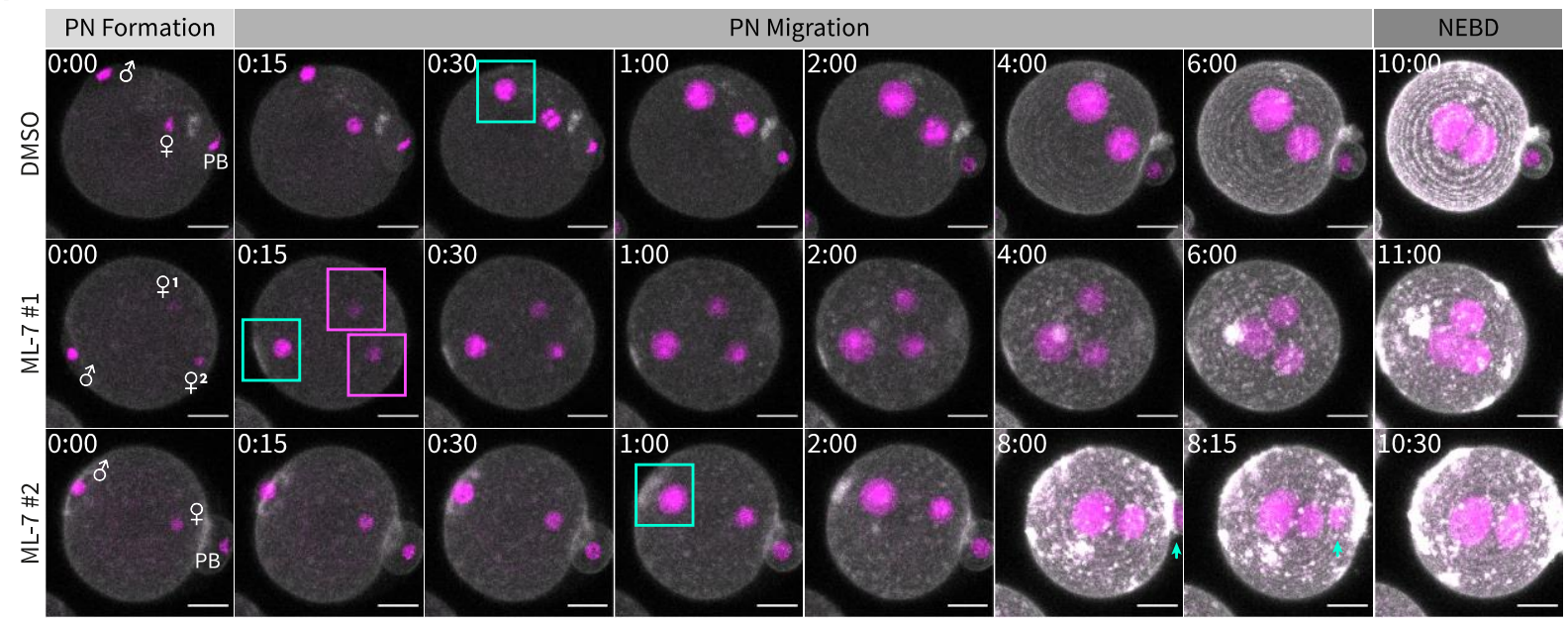

B

Migration of male pronucleus

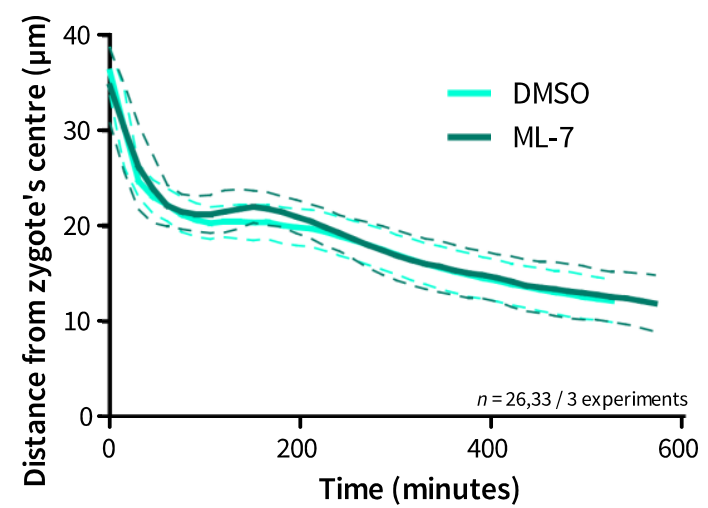

C

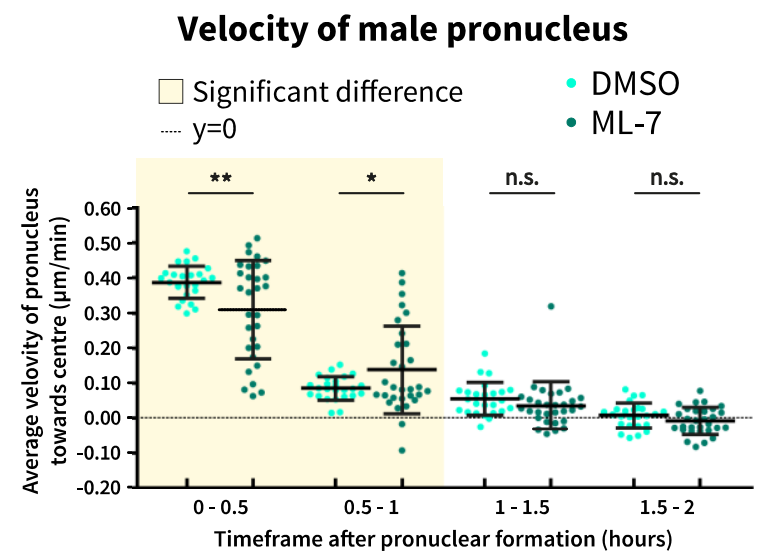

Migration of female pronucleus

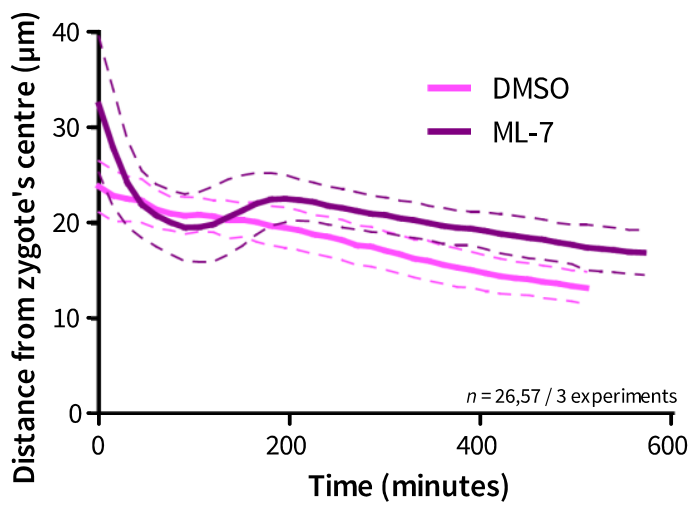

Velocity of female pronucleus
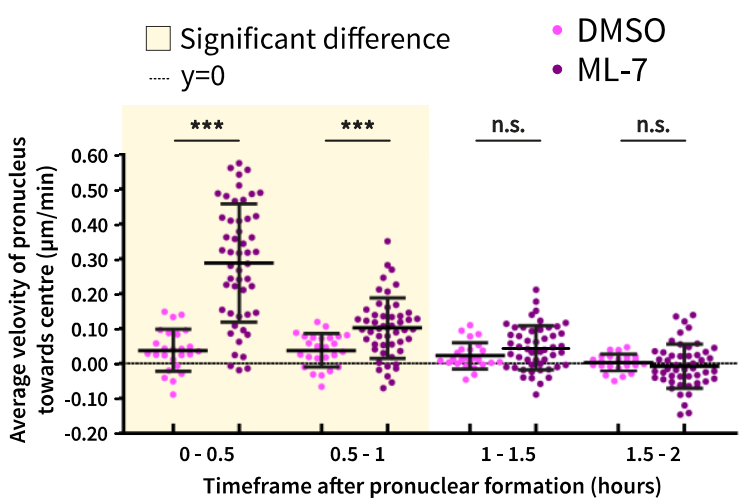
Figure 23: Fast pronuclear migration is possible without actomyosin contractions of the fertilisation cone. (A) Confocal images of live zygotes treated with DMSO or ML-7, expressing MyrGFP (grey) and H2B-mCherry (magenta) mRNA. Cyan box highlights position of male pronucleus as it migrates away from cortex; magenta box highlights position of female pronuclei when the polar body fails to form. Images taken every 15 minutes, every $3 \mu \mathrm{m}$ throughout the zygote. $P B=$ Polar body, $\sigma^{7}=$ male pronucleus $q=$ female pronucleus. Images shown are maximum intensity projections of the z-planes with a 1 px Gaussian blur filter applied. Images taken on Zeiss LSM-880 confocal microscope. Scale bar $=20 \mu \mathrm{m}$. Time shown in hours:minutes relative to pronuclear formation. Representative images; two examples are shown for $M L-7$ as formation of polar body depended on exact time of drug addition. Total number of zygotes $n=26$ for DMSO, and $n=33$ for ML-7 from three experiments. (B) Migration of female and male pronucleus over time. The live imaging data were reconstructed in 3D using IMARIS software, and the relative distance of each pronucleus from the centre of the zygote was calculated and plotted over time. On the left, migration of male pronucleus is shown, and on the right, the female, each comparing zygotes treated with DMSO (light cyan and light magenta respectively), and ML-7 (dark turquoise and dark purple respectively); dashed lines = one standard deviation. (C) Velocity of male (left) and female (right) pronucleus in different time frames. The mean velocity of each pronucleus for each time frame was calculated and plotted so that each dot represents one pronucleus; the mean of all pronuclei for each time frame was calculated and is indicated by the black line with error bars signifying one standard deviation. The colour key is identical to (B). Note the differing $y$-axes for male and female pronuclei, and that the dotted black line shows where $y=0$. Shaded yellow area signifies time frame in which there is a significant difference between the DMSO and ML-7 groups. Statistical significance between conditions calculated with Unpaired, two-tailed t-test with Welch's correction. ${ }^{*} p \leq 0.05, * * p \leq 0.01, * * * p \leq 0.001$.

\section{Spire2 accumulation at the forming male pronucleus persists when actomyosin contractions are} inhibited

In Figure 17, we had described a ring-like accumulation of mClover3-Spire2 at the base of the fertilisation cone; this ring contracted over time to focus over the forming male pronucleus. As contractions of the fertilisation cones have been described around the time of pronuclear formation, with actin and myosin-II localisation described at the base of the fertilisation cone, and the formation of fertilisation cones as well as their contractions were disrupted by inhibiting MLCK function (Ajduk et al., 2011), I next wanted to test if the dynamics of the mClover3-Spire2 localisation were linked to these fertilisation cone contractions. As shown in Figure 23, the fast migration of pronuclei away from the cortex was still possible, though slightly slowed and lengthened, in the presence of ML-7. Next, zygotes expressing mClover3-Spire2 mRNA treated with either DMSO or ML-7 were imaged with high temporospatial resolution at the forming male pronucleus. The results are shown in Figure 24.

In Figure 24A, representative images from a zygote treated with DMSO (top) and a zygote treated with ML-7 (bottom) are shown over time. In the DMSO-treated zygote, a fertilisation cone formed above the forming male pronucleus, and mClover3-Spire2 was clearly visible, as indicated by the cyan arrows. This accumulation concentrated at the cortical region directly overlying the growing male pronucleus, which moved quickly away from the cortex. In the ML-7-treated zygote, no clear protrusion of the fertilisation cone is visible, an effect of ML-7 that had been described in approximately half of the zygotes previously (Ajduk et al., 2011). However, as indicated by the cyan arrows, mClover3-Spire2 is present in these zygotes, and shows similar dynamics as in the DMSO group. The shape of the accumulation appeared slightly different than in control cells, as it was less evenly distributed, possibly 
as a consequence of the fertilisation cone changes in presence of ML-7. The male pronucleus formed and migrated quickly away from the cortex, as in the DMSO-treated zygote shown.

The quantification from three experiments are shown in Figure 24B. All zygotes from both DMSO (14/14) and ML-7 (21/21) groups showed enrichment of mClover3-Spire2 at the forming male pronucleus ( $p>0.05$, two-tailed Fisher's exact test).

This experiment, in conjunction with the results of Figure 23, reveal that MLCK function at the fertilisation cone did not appear to be required for the fast migration of pronuclei away from the cortex, nor for the mClover3-Spire2 accumulation. 

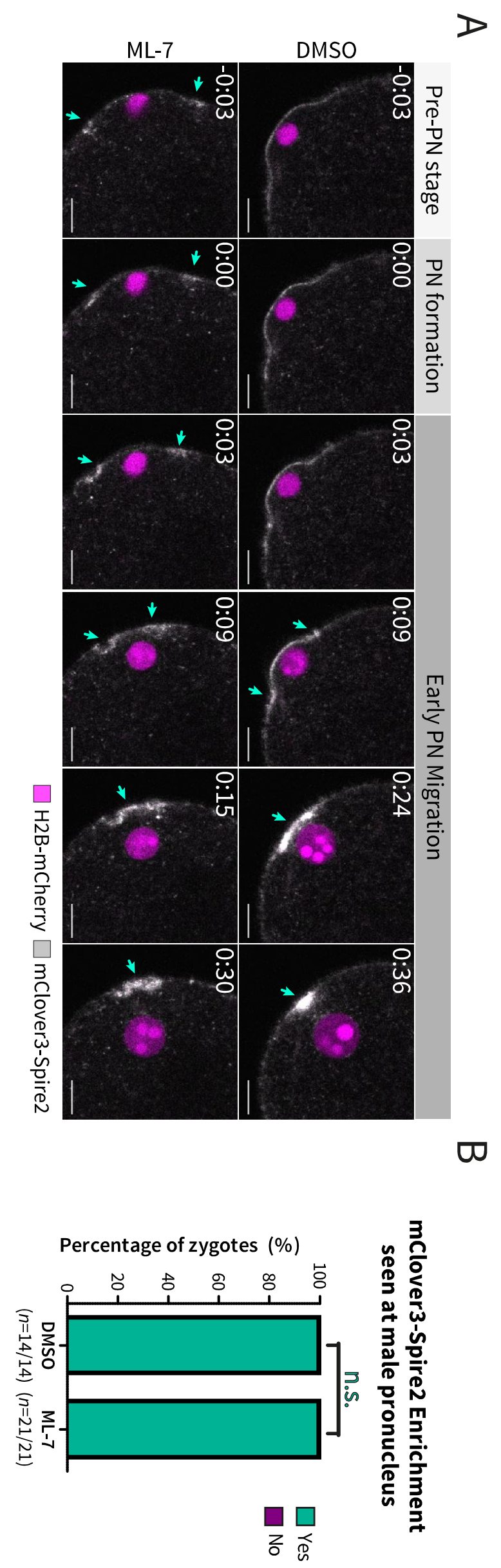

Page 126 of 177 
Figure 24: $\mathrm{mClover3-Spire2}$ accumulation persists when actomyosin contractions are inhibited with ML-7. (A) Confocal images of male pronucleus forming close to the cortex of live zygote expressing mClover3-Spire2 (grey) and H2B-mEGFP (magenta), treated with either DMSO (top) or ML-7 (bottom), over time. Single z-planes of plane at which male DNA is central is shown. Cyan arrow indicates Spire2 accumulation as the pronucleus forms adjacent to the cortex. Images taken with a Zeiss LSM-800 microscope. Gaussian blur $1 \mathrm{px}$ applied. Scale bar $=10 \mu \mathrm{m}$. Time shown in hours:minutes relative to pronuclear formation. Representative images from three experiment are shown, with total $n=14$ for DMSO, and $n=21$ for $M L-7$. (B) Quantification of enrichment seen at male pronuclei shown in (A). Presence of mClover3-Spire2 enrichment was judged by eye, with a yes/no read-out. Two-tailed Fisher's exact test used to test statistical significance; ${ }^{*} p \leq 0.05, * * p \leq 0.01, * * *$ $p \leq 0.001$. 


\subsection{Actin is absolutely essential for pronuclear migration in later pronuclear migration}

Overall, our results so far indicated that Rab11a- and Spire2-related actin nucleation are likely important in the initial stages of pronuclear migration. As shown in the Introduction section 1.4, pronuclei of cytochalasin D-treated zygotes were not able to migrate away from the cortex. It was, however, not clear whether F-actin was only required for pronuclei to migrate away from the cortex, or also for later stages of pronuclear migration, which are not dependent on Rab11a actin vesicles or Formin-2/Spire2-interaction. Therefore, I next investigated the effect of cytochalasin $D$ when it was added after pronuclear formation, and thus after Rab11a- and Spire2-related fast migration away from the cortex. To achieve this, zygotes expressing MyrGFP and H2B-mCherry were imaged from fertilisation. When all pronuclei had formed and the initial migration away from the cortex had occurred, either DMSO or cytochalasin D was added to the imaging dish, and the zygotes were returned to the microscope. These results are shown in Figure 25.

Representative images of zygotes treated with either DMSO or cytochalasin D are shown in Figure 25A. Note that for this experiment, the time could not be set relative to pronuclear formation, but rather, relative to the time point imaging was re-started just after drug addition. In both zygotes shown, male and female pronuclei formed as normal, with the male pronucleus forming at the cortex and migrating quickly inwards, and the female pronucleus forming deeper inside the cell, prior to drug addition. After drug addition, the pronuclei in the DMSO group continued to migrate towards the centre, while in the zygotes to which cytochalasin D was added, the pronuclei stopped migrating towards the centre, and remained in their position as they grew, until the time point of nuclear envelope breakdown.

This migration is quantified in Figure 25B. The migration of both male (left) and female (right) pronuclei is shown, relative to the time of imaging restart after drug addition, indicated by the beige dashed line. Before drug addition, male pronuclei migrated quickly and female pronuclei slowly towards the centre. Then, imaging was paused while the drug was added and zygotes re-positioned, and imaging was re-started. From this point onwards, neither male nor female pronuclei show much if any migration inwards in the cytochalasin D group compared to the DMSO group. At the time point imaging was re-started after drug addition (time point 0), male pronuclei in the DMSO group were at a mean distance of $19.59 \pm 2.98 \mu \mathrm{m}$ from the centre, compared to $22.42 \pm 3.35 \mu \mathrm{m}$ when cytochalasin D had been added ( $p<0.001$, Welch's t-test); evidently, pronuclei in the DMSO group had already continued their migration before imaging was re-started compared to cytochalasin $D$. At the last time point before nuclear envelope breakdown, male pronuclei had migrated to $10.99 \pm 2.03 \mu \mathrm{m}$ from the centre in DMSO-treated zygotes, compared to $19.37 \pm 3.13 \mu \mathrm{m}$ in the cytochalasin $\mathrm{D}$ group 
$(p<0.001, W e l c h ' s t-t e s t)$. The same trend was observed for the female pronuclei. After the drug was added and imaging re-started, female pronuclei in the DMSO group were at a mean distance from the centre of $18.71 \pm 1.77 \mu \mathrm{m}$ compared to $20.11 \pm 2.37 \mu \mathrm{m}$ in the cytochalasin $\mathrm{D}$ group $(p=0.0101$, Welch's t-test). By the end of imaging at nuclear envelope breakdown, female pronuclei had migrated to $10.27 \pm 1.99 \mu \mathrm{m}$ from the centre in DMSO-treated zygotes, but only to $17.47 \pm 1.88 \mu \mathrm{m}$ in those treated with cytochalasin $D(p<0.001$, Welch's t-test).

Next, the average velocities in different time frames were calculated and are shown in Figure 25C. Here, no significant difference was seen between groups before drug addition, but every time frame analysed after drug addition was significantly slower in the cytochalasin D group than in the DMSO group. For example, in the first hours after drug addition, the male pronucleus moved at a mean velocity of $0.035 \pm 0.013 \mu \mathrm{m} /$ minute towards the centre in the DMSO group, and in the cytochalasin D group the mean velocity was $0.011 \pm 0.000 \mu \mathrm{m} /$ minute $(p<0.001$, Welch's t-test). In this time frame, the female pronucleus moved at a mean velocity of $0.036 \pm 0.014 \mu \mathrm{m} /$ minute in the DMSO-treated zygotes, compared to $0.007 \pm 0.006 \mu \mathrm{m} /$ minute in the dominant-negative group ( $p<0.001$, Welch's ttest). This trend was also observed in the later time frames; both male and female pronuclei continued to migrate at a steady pace in DMSO-treated zygotes, compared to near-zero velocities in cytochalasin D-treated zygotes, consistently with a significant difference between the two groups.

These experiments revealed F-actin was important not only in the early, fast migration away from the cortex, but also for later migration of both female and male pronuclei towards the centre. 
A

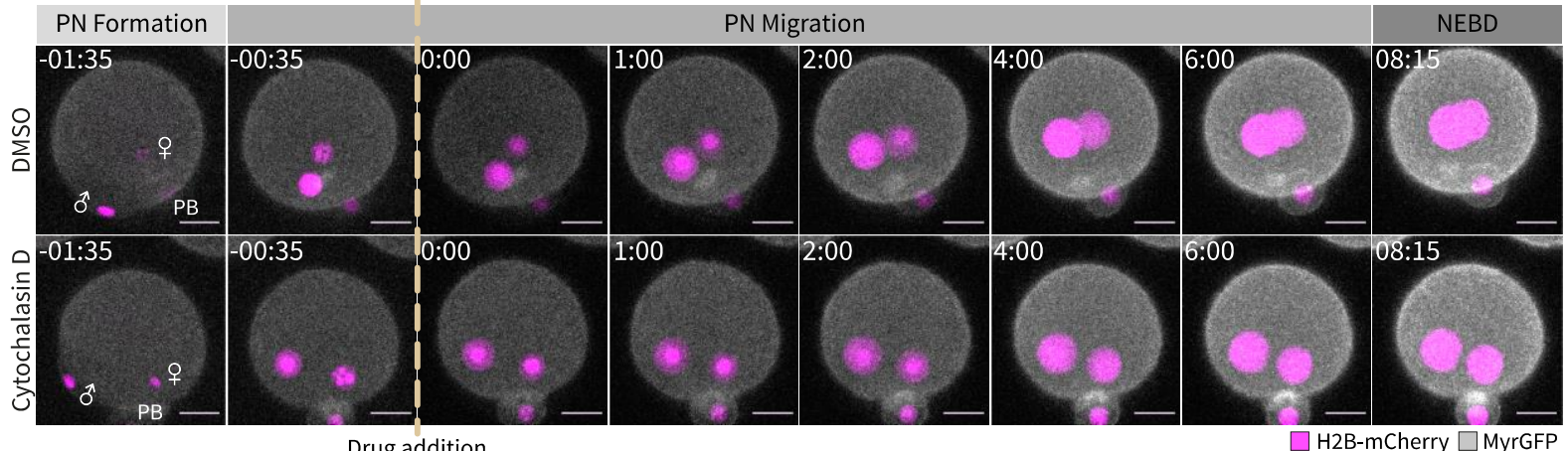

B

Drug addition

$\square$ H2B-mCherry $\square$ MyrGF

Migration of male pronucleus

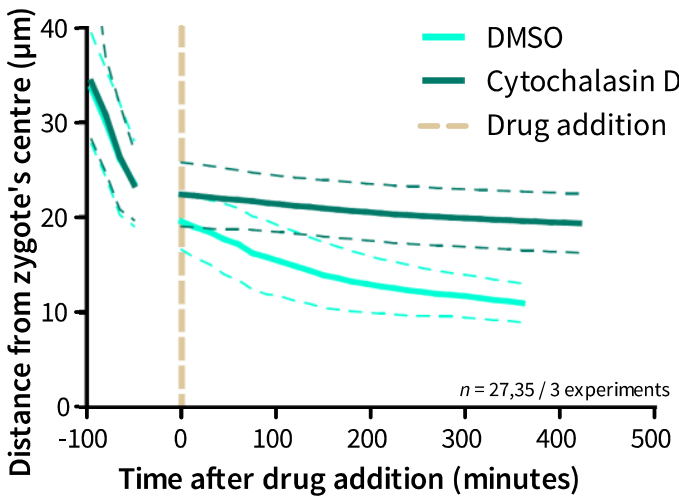

C

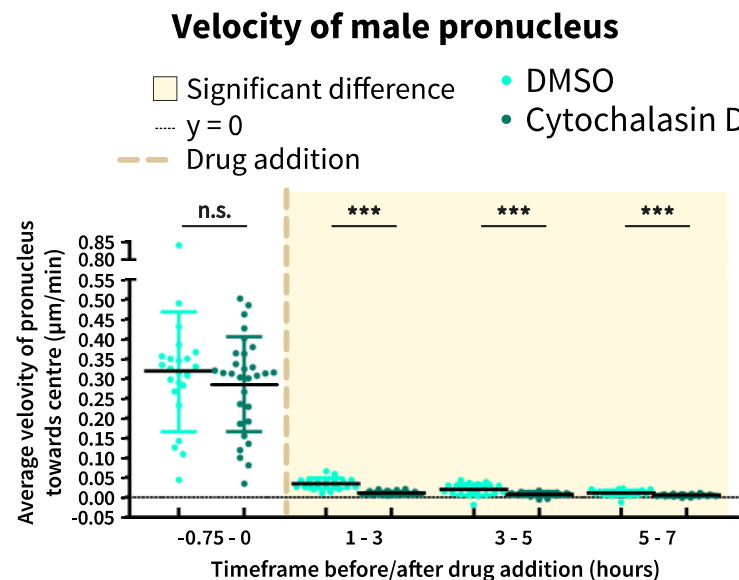

Migration of female pronucleus

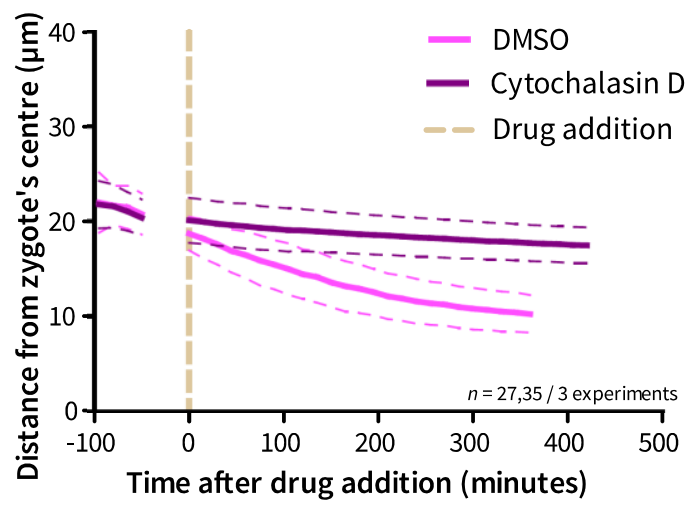

\section{Velocity of female pronucleus}

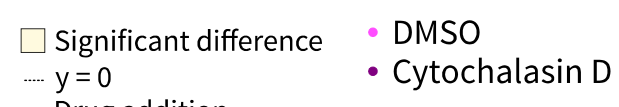

- Drug addition

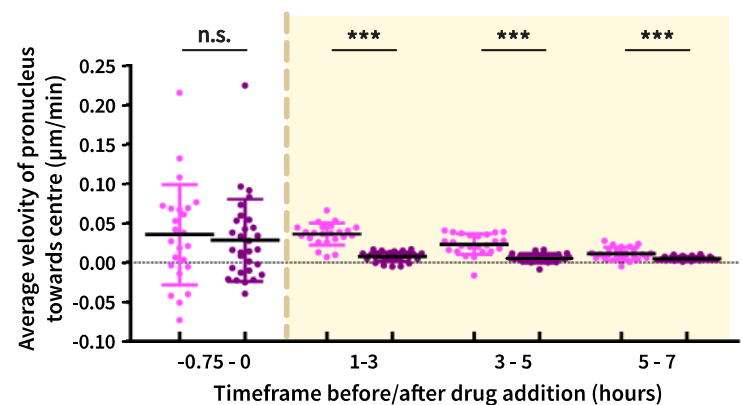

Figure 25: Late pronuclear migration requires F-actin. (A) Confocal images of live zygotes, treated with either DMSO or cytochalasin D after early pronuclear migration was complete, expressing MyrGFP (grey) and H2B-mCherry (magenta) mRNA. Zygotes were imaged until pronuclei had formed and begun migrating; then, either DMSO or cytochalasin D was added, zygotes were re-positioned, and imaging re-started. Images taken every 15 minutes (except during drug addition), every $3 \mu \mathrm{m}$ throughout the zygote. $P B=$ Polar body, $\sigma^{\prime \prime}=$ male pronucleus $q=$ female pronucleus. Images shown are maximum intensity projections of the z-planes with a 1 px Gaussian blur filter applied. Images taken on Zeiss LSM-880 confocal microscope. Scale bar $=20 \mu \mathrm{m}$. Time shown in hours:minutes relative to re-starting of imaging after drug addition, indicated by beige dashed line. Representative images; total number of zygotes $\mathrm{n}=27$ for $D M S O$, and $\mathrm{n}=35$ for cytochalasin $D$, from three experiments. (B) Migration of female and male pronucleus over time. The live imaging data were reconstructed in $3 D$ using IMARIS software, and the relative distance of each pronucleus from the centre of the zygote was calculated and plotted over time. On the left, migration of male pronucleus is shown, and on the right, the female, each comparing zygotes treated with DMSO (light cyan and light magenta respectively), and cytochalasin D (dark turquoise and dark purple respectively); dashed coloured lines = one standard deviation. Beige dashed line = time of drug addition. (C) Velocity of male (left) and female (right) pronucleus in different time frames. The mean velocity of each pronucleus for each time frame was calculated and plotted so that each dot represents one pronucleus; the mean of all pronuclei for each time frame was calculated and is indicated by the 
black line with error bars signifying one standard deviation. The colour key is identical to (B). Note the differing y-axes for male and female pronuclei, and that the dotted black line shows where $y=0$. Shaded yellow area signifies time frame in which there is a significant difference between the DMSO and cytochalasin D groups. Statistical significance between conditions calculated with Unpaired, two-tailed t-test with Welch's correction. ${ }^{*} p \leq 0.05,{ }^{* *} p \leq 0.01,{ }^{* * *} p \leq 0.001$. 
Mechanisms of pronuclear migration in mammalian zygotes: Results

This page is intentionally left blank 


\section{DISCUSSION}

\subsection{Summary of results}

Before I joined this project, Kathleen Scheffler had made a number of fundamental observations about pronuclear migration in mouse zygotes: that male and female pronuclei show different migration dynamics; that filamentous actin is required for pronuclear migration; that microtubules are only important for the slower, later phase of migration; and that brefeldin A inhibits only the early, fast migration of male pronuclei.

My objective for my PhD research was to research in more detail the underlying mechanisms of this fast migration in the first phase of pronuclear migration. Specifically, the localisation and function of Rab11a-positive vesicles as well as Spire- and Formin-type actin nucleators have been investigated, and in this thesis, the results obtained from a variety of observational and functional experiments have been described. On an observational level, fluorescently tagged Rab11a, Spire2 and Formin-2 were all found to localise to the cortical region overlying the forming male pronucleus, with Rab11a localisation described in the most detail using imaging of live and fixed cells. Different perturbation and overexpression experiments revealed that these proteins also play a functional role in the fast migration of pronuclei in their first phase away from the cortex. Interestingly, accumulation of Rab11a and Spire2, as well as fast pronuclear migration away from the cortex was not dependent on the site of sperm entry or actomyosin contractions of the fertilisation cone. As Rab11a, Spire2 and Formin-2 only appeared to be involved in early pronuclear migration, the effect of actin perturbation on later migration was also investigated, and it was found that actin is indeed essential throughout pronuclear migration.

In this next section, the main results leading to these conclusions will be summarised, and where applicable, limitations of the types of experiments used to reach these conclusions will be discussed.

\section{Rab11a, Spire2 and Formin-2 are present at and required for the fast, early pronuclear migration away from the cortex}

The first part of the Results focused solely on the function and localisation of Rab11a throughout zygotic progression. As shown in the introduction, Kathleen Scheffler had previously found that treatment with brefeldin A, which inhibits Golgi function and thus Rab11a vesicle formation, slowed down the initial migration of male pronuclei. As we found the same result in zygotes expressing the dominant-negative form of Rab11a, Rab11a ${ }^{\text {S25N }}$ (Figure 5), we conclude that Rab11a is required for the fast, early migration away from the cortex, but is not essential for later pronuclear migration. 
Optimisation and subsequent imaging of the newly cloned and synthesised mScarlet-Rab11a mRNA in live cells revealed that Rab11a-positive vesicles appeared at fertilisation, increased in number around the time of pronuclear formation, and were gone within about an hour of pronuclear formation (Figures 6 and 7). Optimisation of a commercial Rab11a antibody for immunofluorescence combined with an automated counting method confirmed that endogenous Rab11a-positive vesicles showed the same trend (Figures 8-11). Furthermore, this immunofluorescence and analysis approach allowed confirmation of brefeldin $A$ and Rab11a ${ }^{525 N}$ both perturbing these levels of endogenous Rab11apositive vesicles (Figure 12). It should be noted that the automated counting method I established may still not be perfectly accurate, as volume thresholds had to be set, and it is possible that some non-true vesicles were counted while some true vesicles were not; therefore, absolute number of vesicles in the region analysed may not be precise. Also, an element of circular logic remains in the conclusion that the antibody is indeed specific; the overexpressed fluorescent marker and perturbations were shown to work by using the antibody that was confirmed by co-localisation with the same marker and perturbations. However, it is a somewhat unlikely coincidence that overexpressed Rab11a with different fluorescent markers and the antibody all localise to the same vesicle-like structures that also happen to be significantly perturbed with two different perturbation approaches. Thus, I make the assumption that the antibody is specific, and conclude that Rab11apositive vesicles are present in the cytoplasm at the same time that their perturbation slows down the migration of the male pronucleus.

One observation from live imaging that could not be confirmed by immunofluorescence was the transient accumulation of mScarlet-Rab11a seen at the cortical region overlying the forming male pronucleus. As EGFP-Rab11a observed in the cortical region moments before fixation was not observed in post-fixation imaging, even though cytoplasmic EGFP-Rab11a signal remained, it was not possible to conclude whether Rab11a endogenously accumulated in this region (Figure 10). The preand post-fixation permeabilisation steps are part of the fixation protocol for oocytes and zygotes to allow optimal staining, but it is highly likely that this permeabilisation disrupted membrane structures. This is something that could be optimised in the future, for example by attempting different fixation protocols that have been optimised for membranous structures in other cell types (Stanly et al., 2016). To investigate further what can be observed using live imaging, we decided to optimise high temporospatial resolution imaging of the area surrounding the male pronucleus at formation for fluorescently tagged Rab11a, as well as Spire2 and Formin-2, which are known to co-localise with Rab11a in mouse oocytes (Holubcová et al., 2013). For this, I first optimised high temporospatial resolution imaging of mScarlet-Rab11a, which revealed a transient mScarlet-Rab11a accumulation at the forming male pronucleus in most zygotes imaged (Figure 13). Quantification of this was somewhat 
tricky and subjective, as even a slight rotation of the zygote could make it hard to judge. We did consider automated quantification of this accumulation, and even attempted to measure the intensity of mScarlet-Rab11a in the fertilisation cone over time using regions of interest and kymographs (not shown). As rotation and movement of the zygotes as well as variability in expression between zygotes did not allow automated quantification with the tools available at this time, we found manual quantification by eye to remain our best option. Importantly, the tools used to perturb Rab11a function leading to a slow-down of early pronuclear migration, brefeldin A and Rab11a ${ }^{525 N}$, both appeared to perturb this transient accumulation of mScarlet-Rab11a (Figure 14). However, both brefeldin $A$ and Rab11a ${ }^{525 N}$ made the already-tricky quantification more difficult. In the case of brefeldin A treatment, fluorescent clusters were observed throughout the cell, including in proximity to the fertilisation cone. For the dominant-negative Rab11a $a^{525 N}$, control cells were injected with nonfluorescently-tagged Rab11a, resulting in a competition with mScarlet-Rab11a and a weaker signal. Due to these effects, this experiment was not repeated further.

Next, preliminary data indicated that mCherry-Spire2 also accumulated behind the male pronucleus as it forms, but fluorescently tagged Formin-2 was visible there only when mCherry-Spire 2 was cooverexpressed at higher levels (Figure 15). As co-expression of mEGFP-Spire2 and mScarlet-Rab11a also appeared to change the dynamics of Rab11a accumulation, it seems that Spire2 modulates the dynamics of both Formin-2 and Rab11a, although no clear reverse effect was apparent in these preliminary data (Figure 16). Kathleen Scheffler later used these preliminary results to co-express and image Spire2 and Formin-2 together, which is shown in the appendix (Figure 29). Next, I cloned and synthesised mClover3-Spire2 mRNA, and zygotes expressing this were imaged in the same way as for mScarlet-Rab11a by Kathleen Scheffler (Figure 17). The mClover3 fluorophore was better suited for this imaging, as it allowed clearer observation of the dynamics of Spire2. While all three fluorescently tagged proteins appeared to localise to this area at some point around the time of pronuclear formation, the exact localisation and dynamics differed. Rab11a was first observed as a thin layer in the fertilisation cone membrane, which became brighter directly overlying the forming pronucleus. Spire2 was observed as a ring-like accumulation at the base of the fertilisation cone, which constricted to form a bright spot as the pronucleus migrated away. As shown in the Appendix, when Formin-2 was co-expressed with Spire2 (Figure 29), it was excluded from the fertilisation cone until its constriction, and was then accumulated by the forming male pronucleus. While it would be interesting to visualise these proteins simultaneously in the same cell in order to learn more about the real-time differences in the dynamics of these proteins, this would require co-expression, which can modulate the observed localisation and dynamics. It should be noted that the individual imaging of mScarlet-Rab11a and mClover3-Spire2 still required artificial overexpression, and although concentrations of mRNA were 
kept as low as possible, we cannot exclude that the overexpression itself would affect the observed localisation.

As Formin-2 and Spire2 interact to nucleate actin in other contexts, we used several different methods to investigate whether they were nucleating actin at this site. Imaging of actin live probes caused cytoskeletal defects and/or did not allow detection of individual filaments over time (Figure 18), and therefore, we used a functional approach to disrupt actin nucleation by Formin-2 and Spire2. Indeed, perturbation of Formin-2/Spire2 interaction at the FH2-KIND domain by injection of purified FH2 inhibited the fast migration of pronuclei as seen for brefeldin A and Rab11a ${ }^{525 N}$ (Figure 19), and perturbed the accumulation of Spire2 (Figure 20). Thus, we conclude that actin nucleation through Formin-2 and Spire2 is required for the fast migration of pronuclei. Unfortunately, one issue with the perturbation methods used is that Rab11a, Spire2, and Formin-2 are all required for the dynamic cytoplasmic actin mesh in oocytes along which the Rab11a-positive vesicles travel to the plasma membrane (Holubcová et al., 2013; Pfender et al., 2011; Schuh, 2013); therefore, perturbation of any of these would not only perturb the cortical, transient accumulation we observed, but also the cytoplasmic actin network. Thus, it was difficult to conclude whether fast pronuclear migration was dependent on the cortical, transient accumulations of these proteins, or the cytoplasmic mesh from these results. As we are not aware of an experimental approach to target only the cortical accumulation of these proteins or actin, we instead used an overexpression approach to verify a function of localised actin nucleation. Indeed, higher levels of Spire2 were sufficient to elongate migration away from the more prominent Spire2 accumulation at the overlying cortex, sometimes even of both pronuclei to the other side of the cortex (Figure 21); this migration was also quantified by Kathleen Scheffler (appendix Figure 30). As the pronuclei migrated away from the cortical accumulation of Spire2 past the centre of the cell, we conclude that this strong cortical accumulation of Spire2 was the cause of this elongated migration. We conclude that the accumulation of Rab11a, Spire2 and Formin-2 by the forming male pronucleus drive fast migration away from the cortex, possibly through actin nucleation at the site.

\section{Fast pronuclear migration away from the cortex is not dependent on sperm entry site or the}

\section{fertilisation cone}

As Rab11a, Spire2 and Formin-2 accumulated transiently at the cortical region of the contracting fertilisation cone, we wondered whether this localisation and the fast migration were dependent on the sperm entry site and/or the nature of the fertilisation cone. In this context, an early preliminary result revealed that sperm DNA located deeper in the cortex at formation did not appear to migrate as fast, and Rab11a accumulation was not observed as strongly, if at all (Figure 13 and Kathleen Scheffler's initial results). However, the depth of sperm penetration into the egg could not be 
experimentally controlled, and therefore, the relationship between the distance of the pronucleus from the cortex and the intensity of accumulation could not be quantified with this approach. We instead used the microtubule-depolymerizing drug nocodazole to allow maternal DNA to move to the cortex and form a pronucleus there. While we can only speculate about the mechanisms by which the maternal DNA migrates to the cortex, it is possible that there are cortical flows similar to those observed in oocytes (Yi et al., 2011), and the maternal DNA may be subjected to these flows when the spindle remnant is depolymerised, bringing it closer to the cortex. Importantly, Kathleen Scheffler had found that female pronuclei forming at the cortex showed a fast inward migration (appendix figure 28). High temporospatial resolution imaging revealed that a protrusion formed overlying the cortical maternal DNA, and both Rab11a and Spire2 accumulated as the female pronucleus formed (Figure 22), as was previously seen for the male pronucleus (Figure 13 and 17). I thus conclude that fast migration away from the cortex is not dependent on sperm entry site, but rather on the proximity of the DNA to the overlying cortex.

A previous study described rhythmic actomyosin-dependent contractions of the fertilisation cone that ceased at pronuclear formation (Ajduk et al., 2011), and actomyosin contractions have been shown to be involved in nuclear migration in various contexts (Martini and Valdeolmillos, 2010; Schenk et al., 2009; Tsai et al., 2007). Several observations made in our experiments suggested a possible link between pronuclear migration and these actomyosin contractions: first, they occurred at the appropriate time point to be functionally relevant; second, the ring-like localisation of mClover3Spire2 was similar to the described actin and myosin localisation at the base of the fertilisation cone (Ajduk et al., 2011); and third, a fertilisation-cone-like protrusion and the same Spire2 and Rab11a enrichment were seen when female pronuclei formed close to the cortex. Hence, I wondered whether actin nucleation by Spire2 and Formin-2 could be required for these actomyosin contractions of the fertilisation cone, as opposed to actin nucleation itself acting as the main source of force for fast pronuclear migration. I therefore inhibited MLCK activity with ML-7 (Figure 23), although culture conditions were suboptimal as an oil overlay could not be used to avoid evaporation of the medium, and due to the required high DMSO concentration to reach the ML-7 concentrations as used previously in oocytes and zygotes (Ajduk et al., 2011; Schuh and Ellenberg, 2008). The fast pronuclear migration was only slightly delayed as a result, but male pronuclei travelled as far within the first hour without myosin-II function as in control cells. Furthermore, female pronuclei sometimes formed at the cortex due to failure of polar body extrusion, and as a result, migrated quickly inwards. Fertilisation cone protrusions were not as pronounced as in control cells, but mClover3-Spire2 accumulation persisted (Figure 24). As mentioned, culture conditions in this experiment were not ideal, as $30 \mu \mathrm{M}$ concentration of ML-7 required a high concentration of DMSO, and the dish could not be covered with 
oil. However, ML-7 activity was confirmed by co-incubating oocytes in the same dish during imaging, and found that these failed to extrude polar bodies. Furthermore, zygotes treated with ML-7 were not able to form polar bodies or undergo the first mitotic division. Thus, while myosin-II is likely to be important for many functions of the cell, I conclude that it does not seem to be essential for fast pronuclear migration away from the cortex. It is more likely that actin nucleation by Spire2 and Formin-2, facilitated by Rab11a, or Rab11a vesicle accumulation facilitated by Spire2 and Formin-2, may be the main driver of this fast migration.

\section{Actin is absolutely essential for early and late pronuclear migration}

As mentioned in the introduction, treatment of zygotes with cytochalasin $D$ completely perturbs pronuclear migration. We showed that perturbing actin-related proteins Rab11a, Spire2 and Formin2 slowed down only the initial migration of pronuclei, but they were usually still able to reach the centre by the time of nuclear envelope breakdown. Interestingly, injection of purified FH2 into zygotes also slowed down only the early migration phase, even though it perturbed cytoplasmic actin as much as knock-out of Formin-2 in mice ( $\mathrm{Fmn}^{-\%}$ ) (data by Kathleen Scheffler, data not shown). Therefore, I wanted to confirm that F-actin was indeed required for pronuclear migration past the early phase. Indeed, acute addition of cytochalasin D to zygotes in which pronuclei had already formed and migrated from the cortex perturbed any further migration. Thus, it appears that actin structures that are not dependent on nucleation by Formin-2 and Spire2, but are nonetheless depolymerised by cytochalasin D, are important for later migration of the pronuclei. Kathleen Scheffler further investigated this later migration, and taking our results together, we propose a new model for pronuclear migration in mouse zygotes.

\subsection{A new model for pronuclear migration in mouse zygotes}

The overarching theme of this project has been to explore the mechanisms of pronuclear migration in mouse zygotes, and this thesis focused on the evidence that actin nucleation, mediated by Rab11a, Spire2, and Formin-2, was required for the first, fast migration of pronuclei away from the cortex. In parallel, Kathleen Scheffler focused her investigations mostly on the mechanisms of the slower migration phase. How these combined findings fit in with previous models of pronuclear migration, and what model we now suggest, will be discussed here.

As described in the introduction, in most species studied, pronuclei migrate towards each other and the centre of the cell in a microtubule- and sperm-aster-dependent manner. In mice, mature sperm lack centrioles, and zygotes depend on actin to migrate their pronuclei to the centre. Specifically, a model had been proposed in which a gradient of actin vesicles propels both pronuclei towards the centre. Actin vesicles, identified as the nodes of EGFP-Utrophin, were described to be enriched 
between the pronucleus and cortex throughout the 12-15 hours of pronuclear migration. Inhibition of the actin cytoskeleton or myosin- $\mathrm{Vb}$ disrupted migration of pronuclei towards the centre, and a role for microtubules was mostly disregarded in this model (Almonacid et al., 2018; Chaigne et al., 2016). Several mechanistic details were missing from these results, such as the identity of the actin vesicles and nucleators responsible for this pushing force. Furthermore, it was not clear from the results whether these unidentified actin vesicles were the only mechanism at play, as inhibition of myosin-Vb caused severe shape deformations, making it difficult to quantify the effect as compared to cytochalasin D.

While the mentioned study referred only to actin vesicles, visualised with the actin-binding EGFPUtrophin (Chaigne et al., 2016), we had reason to believe that these may in fact be Rab11a-positive vesicles. In GV oocytes, the same research group described such actin vesicles as forming nodes of the cytoplasmic actin mesh required for nucleus centration in a Formin-2- and myosin-Vb-dependent manner (Almonacid et al., 2015). Previously, our group had described Rab11a vesicles to lie at the actin nodes of GV and MI oocytes, co-localising with and sequestering actin nucleators Formin-2 and Spire2, migrating along actin tracks to the cell periphery in a myosin-Vb related manner (Holubcová et al., 2013; Pfender et al., 2011; Schuh, 2013). Importantly, in contrast to expression of myosin-Vb ${ }^{\text {TAlL }}$ in zygotes (Chaigne et al., 2016), the dominant-negative Rab11a ${ }^{525 \mathrm{~N}}$ variant expressed at the levels performed here did not disrupt cell shape in the majority of cells, and the imaging system established thus allowed precise tracking of pronuclei in the absence of Rab11a-positive vesicles. Perturbation of Rab11a caused a change in pronuclear migration, as the initial faster migration of pronuclei, in particular the male pronucleus, was perturbed. Both expression of Rab11 $11^{\mathrm{S} 25 \mathrm{~N}}$ and injection of purified FH2, disrupting Formin-2/Spire2 interaction, resulted in this phenotype; however, in both cases, pronuclei were later able to continue migration towards the centre, indicating that Rab11a vesicles and Formin-2/Spire2 interaction are not required throughout pronuclear migration. This was consistent with the fact that cytoplasmic Rab11a vesicles appeared at fertilisation but were absent soon after pronuclear formation. Furthermore, our attempts to image actin with EGFP-Utrophin did not confirm the strong accumulation of actin nodes described hours after pronuclear formation (Chaigne et al., 2016), but revealed that overexpression of this construct could lead to artefacts in the cytoplasm. We instead found that Rab11a and its related actin nucleators transiently accumulated at the cortical area overlying the forming male pronucleus. Thus, we firstly propose that the actin vesicles described in the previous model of mouse pronuclear migration (Almonacid et al., 2018) may be Rab11a-positive vesicles, and that these are indeed important for pronuclear migration, but that their function (and that of related actin nucleators Formin-2 and Spire2) is limited to the fast migration phase shortly after pronuclear formation near the cortex. Furthermore, our results indicate that 
accumulation of these proteins and fast pronuclear migration is independent of the sperm entry site, as maternal DNA located near the cortex shows the same enrichment and fast migration away from the cortex (Figure 22). As inhibition of myosin-II and thus actomyosin contractions of the fertilisation cone did not cause the same defect as perturbation of Rab11a or Formin-2/Spire2, we conclude that it is unlikely that these actin nucleators facilitate migration through actomyosin contractions. Rather, fast migration away from the cortex may be facilitated through Rab11a vesicle accumulation or local actin nucleation itself, but we were not able to conclusively distinguish between these two possibilities experimentally. Critically, the last result presented in this thesis showed that while actin vesicles and nucleators tested were not required for later migration of pronuclei, actin itself continued to be important, as acute addition of cytochalasin $\mathrm{D}$ after the fast migration still completely inhibited any further migration. As Kathleen Scheffler had found that disruption of microtubules slowed down this later migration of pronuclei, she investigated how actin and microtubules together facilitate this migration. These results are important for our model of centriole-free pronuclear migration in mouse zygotes, and will be summarised here.

Kathleen Scheffler made key findings that explained how actin and microtubules may interact to facilitate the slow migration phase of pronuclei in the last $\sim 8$ hours before nuclear envelope breakdown. As my work focused on the earlier migration, experiments conducted with or by Kathleen Scheffler critical to this phase are shown in this thesis or its appendix. However, as my contribution to investigations of the later migration was minor (e.g. helping with injections, quantifications, or discussions), detailed figures are not shown, but I will briefly describe the main findings here. Kathleen Scheffler observed that the microtubule network of mouse zygotes was initially sparse, the main structure visible being the spindle remnant. Over time, a dense microtubule network grew, with some pericentrin-positive asters appearing, but no observable clear orientation of microtubules. The pericentrin-positive MTOCs showed an overall inward movement over time, but their disruption with pericentrin TRIM-Away (Clift et al., 2018) did not otherwise affect either the microtubule network or pronuclear migration. On the other hand, disruption of dynein with the dominant-negative P150-CC1 mutant altered the dynamics of the microtubule cytoskeleton, and inhibited inward movement of both MTOCs and pronuclei. Cytochalasin D treatment froze the microtubule network, indicating that actin and microtubules were indeed linked during this stage. High temporospatial imaging of microtubules revealed buckling and bending of microtubules between cortex and pronuclei, possibly providing the key to how actin can facilitate slow migration of pronuclei even in the absence of Formin2/Spire2-nucleated cytoplasmic actin: through its cortex. Critically, this late migration of pronuclei is not passive, as tracking of microinjected oil droplets did not show the same migration patterns. Finally, 
pronuclei appeared flattened on their inner surfaces once they reached the centre, providing further evidence of a pushing force.

These combined results propose a new model of centriole-independent pronuclear migration in mouse zygotes. After fertilisation, the male pronucleus forms closer to the cortex than the female pronucleus. Male and female pronuclei show different dynamics in their migration: the female pronucleus forms and migrates towards the centre at a relatively steady pace, and the male pronucleus is launched from its cortical position inwards at a high velocity in the first hour after pronuclear formation. Rab11a-positive vesicles appear at fertilisation, and, together with actin nucleators Formin-2 and Spire2, transiently localise to the cortical region overlying the male pronucleus. Here, they facilitate the fast migration of the male pronucleus, either directly through their localisation or local actin nucleation, but not through actomyosin contractions of the fertilisation cone. Soon after this first migration phase, Rab11a-positive vesicles disappear from the cytoplasm, and Formin-2/Spire2-related actin nucleation is no longer required for pronuclear migration. Instead, microtubules and dynein coordinate pronuclear migration, though still in an actin-dependent manner; growing microtubules possibly bend against cortical actin, thereby pushing the pronuclei towards the centre. Our model thus describes two separate pathways required for pronuclear migration in mouse zygotes. Localised actin-related vesicles and nucleators first facilitate fast launching from the cortex, and a growing microtubule network then facilitates slow migration to the centre in an actin- and dynein-dependent manner. This new model is shown in Figure 26. 

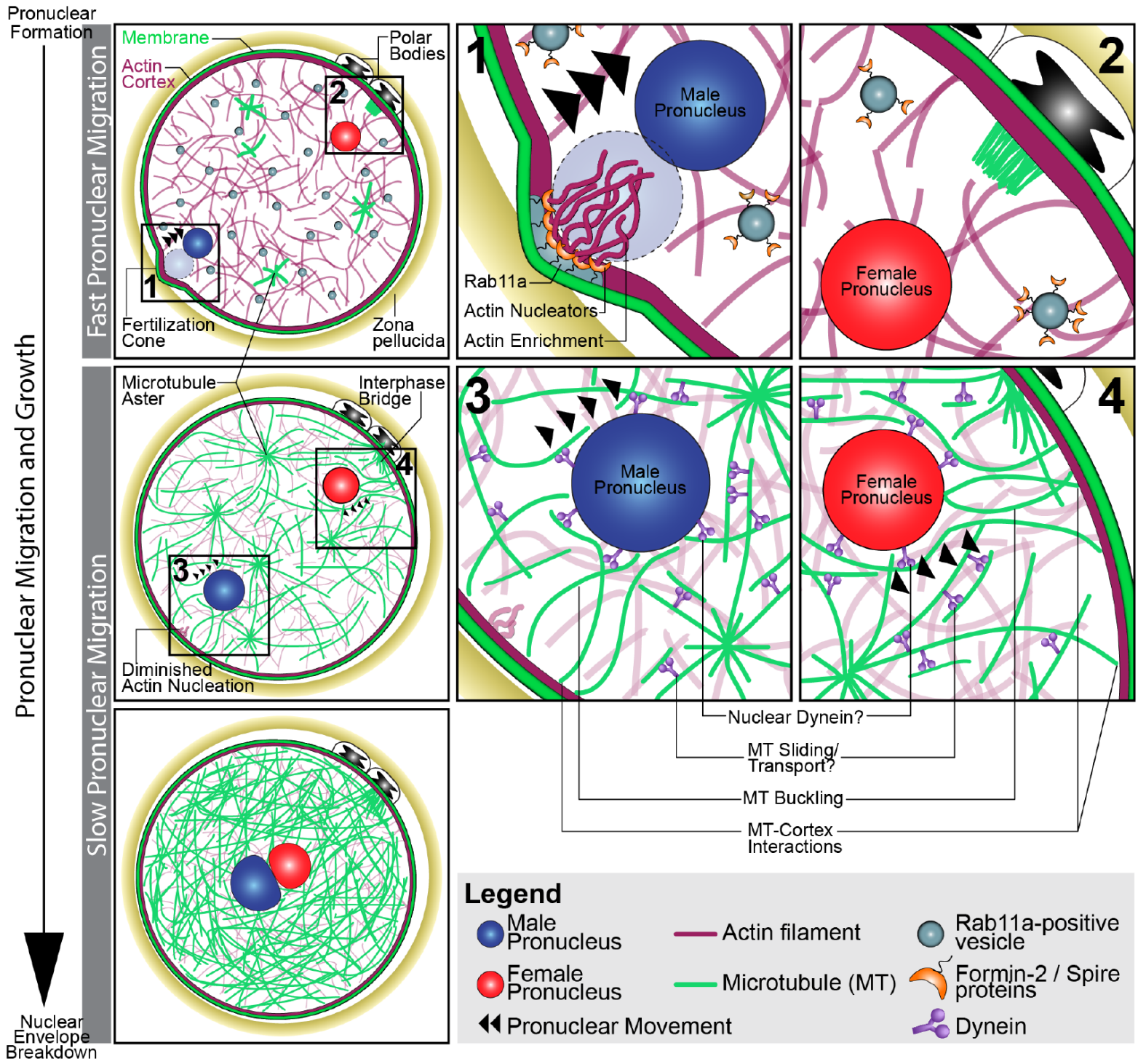

Figure 26: A new model for pronuclear migration in mouse zygotes. Pronuclear migration is initiated by Rab11a-positive vesicles that target Formin-2/Spire actin nucleators to regions of the cell surface adjacent to pronuclei (primarily male pronuclei which assemble close to the cell surface at the site of sperm entry, zoomed-in in panel 1). This promotes short-lived localised actin polymerisation from vesicle-enriched regions that propels fast-phased migration (large arrowheads) of male pronuclei away from the cell surface. In contrast, female pronuclei typically assemble farther away from the cell surface due to remnant microtubules of the meiosis II spindle (green microtubule clusters, zoomed-in in panel 2). This maintains female pronuclei outside the operational range of fast-phased movement. Eventually, pronuclei that are farther away from the cell surface are moved in slow-phased migration mode (small arrowheads) by a dense but highly mobile network of microtubules in a dynein-dependent manner. Together, coordination of actin and microtubules achieves accurate positioning of pronuclei at the centre of zygotes before their disassembly for mitosis. Figure prepared by Kathleen Scheffler for manuscript in preparation. 


\subsection{Open questions \& future experiments to test the model}

We have used new live imaging methods to elucidate the functions of mouse pronuclear migration in far greater detail than had been previously described. This allowed an update to the current model to be proposed, but it has also opened many new questions for further investigation.

First of all, it should be noted that all of the experiments conducted were based on in vitro fertilised eggs from superovulated mice from a specific hybrid strain. The C57BL/6J x CBA/CaOlaHsd F1 mouse strain was used due to hybrid vigour; hybrid strains are more fertile and better-suited for IVF (Silver, 1995). To allow visualisation of specific proteins, many experiments involved overexpression of mRNA in the zygotes. As mouse oocytes are transcriptionally silent, transfection with DNA was not possible for expression of fusion proteins. Therefore, mRNA expression was the best way to allow a wide range of proteins to be investigated without the need to first establish several reporter lines. In the future, it would be interesting to test whether the findings presented in this thesis also apply to other strains of mice, in vitro matured oocytes or zygotes fertilised inside the mouse, as well as zygotes of genetically modified strains in which the endogenous protein is fluorescently tagged.

The experiments shown in this thesis demonstrated that Rab11a vesicles were absent in unfertilised MII oocytes, appearing only at fertilisation. This was a new finding; Rab11a-positive vesicles had previously been described only in GV and MI oocytes using fluorescently-tagged Rab11a (Holubcová et al., 2013; Schuh, 2013). It would be interesting to further investigate the precise timing of Rab11a vesicles loss during meiotic progression, and which regulatory pathways are involved. The Rab11a immunofluorescence and live imaging tools I optimised as part of this project could be applied to investigate this. Understanding the signals that induce Rab11a expression and vesicle formation from fertilisation onwards is another area of further interest. For example, it is possible that Rab11a vesicle formation is induced through the rhythmic calcium signalling induced by sperm entry. The highly dynamic and rhythmic nature of Rab11a vesicles observed when imaging every three minutes indicated that Rab11a vesicle numbers oscillated, possibly with a periodicity of 9-18 minutes; this is similar to the described period of 11-31 minutes of calcium oscillations (Deguchi et al., 2000). Live imaging of Rab11a vesicles with even higher temporal resolution, potentially together with calcium markers that have been used previously, such as calcium green-1 dextran (Deguchi et al., 2000; Kyozuka et al., 2008) or FuraRed (Ajduk et al., 2011), could reveal potential correlations between calcium and Rab11a vesicle oscillations. Furthermore, imaging of mScarlet-Rab11a with higher temporal resolution could reveal possible directionality of Rab11a-positive vesicles, or reveal how Rab11a, Formin-2 and Spire2 accumulate so quickly and transiently at the cortical region overlying the pronucleus. It should be noted that I attempted to image single planes of zygotes expressing $m S c a r l e t-$ 
Rab11a with a time resolution of 3-5 seconds during troubleshooting experiments, and found that individual vesicles were difficult to track due to their migration in different directions at high velocities (data not shown). It may be possible to follow vesicles during live imaging using a tracking macro, such as Autofocuscreen (Rabut and Ellenberg, 2004), MyPiC (Politi et al., 2018) and TipTracker (von Wangenheim et al., 2017), which have been optimised for a variety of moving samples. However, it is possible that the sheer number of vesicles in the cytoplasm would make this difficult. I also attempted self-activation of MII oocytes using strontium chloride (Ma et al., 2005) and subsequent imaging of mScarlet-Rab11a, but lack of real data prevented conclusions to be drawn. Bright mScarlet-Rab11apositive vesicles were seen in the activated oocytes during imaging set-up, but by the time all positions were set up and imaging was started, these vesicles were unfortunately no longer visible (data not shown). In normally fertilised zygotes, vesicles were found in the cytoplasm for several hours after sperm entry until shortly after pronuclear formation, but they persisted here only for approximately 30-45 minutes after addition of strontium chloride to the medium, possibly because of a faster onset of pronuclear formation. Strontium chloride activates eggs to complete meiosis by triggering calcium oscillations (Bos-Mikich et al., 1995; Zhang et al., 2005), and therefore, it is possible that calcium signalling at sperm entry induces Rab11a vesicle formation in the cytoplasm. A more appropriate functional test could be to perturb calcium signalling in fertilised zygotes, for example with roscovitin (Deng and Shen, 2000), to test any causal, mechanistic link between calcium signalling and Rab11a vesicle formation.

The nature of the mScarlet-Rab11a accumulation observed at the cortex overlying the forming pronucleus could be investigated next. It is not clear from the results shown whether this enrichment consists of vesicle-bound Rab11a, or of free Rab11a protein accumulating independent of vesicles. The images shown in this thesis did not have a high enough resolution to judge whether this enrichment consisted of a collection of many small vesicles, or of accumulating, membrane-free protein. Confocal imaging with even higher resolution, or possibly using Airyscan or STED systems, may help to clarify this point. Additionally, electron microscopy of fixed zygotes at this timepoint may be useful in understanding whether membranous vesicles or phase-separated, membrane-free protein is accumulating here. Indeed, electron microscopy was recently used to identify the liquid-like nature of a novel spindle domain in mouse oocytes (So et al., 2019). Tracking of individual vesicles with high temporal resolution, as suggested above, may also help to understand whether vesicles stay intact during their migration to the cortex, or rather release Rab11a protein to transiently accumulate.

Regardless of whether Rab11a accumulated to the cortical region in the form of vesicles or free protein, we were able to demonstrate that fluorescently tagged Rab11a, Spire2 and Formin-2 all transiently localise to the forming male pronucleus, and that both Rab11a function and Formin- 
2/Spire2 interaction are required for fast migration of pronuclei. However, we were not able to investigate the exact hierarchy of this pathway. Firstly, we cannot experimentally distinguish between Rab11a vesicles and sequestered actin nucleators present in the cytoplasm, and the transient accumulations seen at the cortex overlying the pronucleus. This is because a co-dependency appears to exist between Rab11a, Spire2 and Formin-2 that controls their correct dynamics and localisation. Without Rab11a vesicles, actin nucleators are not sequestered properly, leading to changes in the cytoplasmic actin network (Holubcová et al., 2013), and mClover3-Spire2 cannot accumulate to the cortex (data by Kathleen Scheffler, not shown). On the other hand, without Spire2 and Formin-2 actin nucleation, Rab11a-positive vesicles do not have cytoplasmic actin tracks to travel along (Holubcová et al., 2013), and as shown in the results, neither Rab11a nor Spire accumulated at the forming pronucleus when $\mathrm{FH} 2$ was injected. Overexpression of Spire 2 changed the pattern of both Rab11a and Formin-2 localisation with no clear modulation in the other direction, indicating that Spire2 may possibly act upstream of Rab11a and Formin-2 in the transient cortical accumulation; also, Spire2 overexpression is sufficient to elongate fast migration. Our results further showed that fluorescentlytagged Rab11a, Formin-2, and Spire2 all localised to slightly different surface regions of the fertilisation cone, although the exact mechanism or function of this localisation was not further investigated. No location-specific or inducible perturbation of any of these proteins currently exist. It could be interesting to create and optimise a photo-inducible Rab11a ${ }^{525 \mathrm{~N}}$, TRIM-Away (Clift et al., 2018), or other method to perturb function of specific proteins at the cortical region overlying the male pronucleus alone, and to investigate the effects on the other proteins, actin nucleation, and pronuclear migration. For example, such photo-inducible systems have been used to control localised GTPase activity in fibroblast cell cultures (Guntas et al., 2015). However, it is not clear whether spatiotemporal control would be sufficiently precise to make this experimentally feasible in the context of the mouse zygote.

Assuming that cortical accumulation of these proteins is required, important questions remain: are Formin-2 and Spire2 required to allow vesicle localisation to the cortical region adjacent to the pronucleus, and are these vesicles sufficient to create a pressure gradient, as suggested in the previous model of mouse pronuclear migration (Almonacid et al., 2018) - or are Rab11a vesicles required to transport the actin nucleators to the cortical region, where they nucleate actin to facilitate launching of the pronucleus from the cortex? A recent study suggested that Spire2 forms a complex with Rab11 through myosin-V in vitro (Pylypenko et al., 2016), and that this binding facilitates myosin-V and Rab11 activation. It would be interesting to see if this is the case in mouse zygotes, and investigate whether Formin-2 is also associated with this complex. Perturbing this interaction, for example by expressing Spire2 with a mutation at the identified 27-amino-acid-long linker homology region, may provide 
further insight into the functional hierarchy. However, it is likely that this would simply cause the same issue as described above: perturbation of both cytoskeletal and cortical localisation of all three proteins, with no clear answer evident due to the co-dependency. Thus, time- and localisation-specific experimental perturbation of this interaction would still be required.

This hierarchy could also be investigated by testing actin nucleation directly. Imaging of different actin probes was attempted, but it was difficult to draw conclusions from these experiments. Testing further actin probes for high-resolution imaging of actin filaments would be interesting. Several recent studies have used different approaches of visualising newly polymerised actin in live cells, with photoactivatable GFP to track actin filament movement in mouse embryos (Zenker et al., 2018) and by injecting of phalloidin into starfish oocytes to mark already-formed actin networks (Bun et al., 2018). These could be used to investigate whether actin is newly polymerised in the region of Rab11a, Spire2 and Formin-2 accumulation. In addition to testing actin nucleation probes, functional tests could also give additional insight. Actin is required for Rab11a and actin nucleator localisation to the cortical area, and global actin perturbation would therefore perturb both vesicle accumulation and potential resulting actin nucleation. Thus, a localised perturbation of actin nucleation between the pronucleus and the cortical region overlying it would be required. Laser ablation has been used to locally perturb both microtubule and actin filaments in a variety of cell types (Decker and Brugués, 2015; Goulding et al., 2007; Ito et al., 2017; Lomakin et al., 2015); however, this destroys many different structures, and a locally inducible method to perturb actin specifically would be preferable. Colcemid-UV has been used to allow spatial control of microtubules (Hamaguchi and Hiramoto, 1986), but we are not aware of an equivalent light-controlled actin depolymerising drug. However, development of photoinducible control of protein activity (Benedetti et al., 2018; Guntas et al., 2015) may well make such an experiment possible in the future.

As mentioned in the introduction, microtubules and actin can facilitate nuclear migration through a variety of mechanisms, either directly as a pushing mechanism, or through motor proteins and LINC complexes (reviewed by Dupin and Etienne-Manneville, 2011). We were able to exclude a requirement for actomyosin contractions for this fast migration away from the cortex, but we were not able to conclude exactly how the force might be transmitted to the nucleus. It seems feasible that both vesicle accumulation and localised actin nucleation could be sufficient to exert a pushing force behind the pronucleus, and it would be interesting to carry out mathematical modelling of these forces. The fact that pronuclei begin migrating away from the cortex only when they have begun to form and assemble their nuclear envelope might suggest that the nuclear envelope is somehow required for force transmission. Therefore, it is also possible that any growing actin filaments in this region may be coupled directly to the pronuclear envelope, for example through LINC complexes. 
KASH proteins contain actin-binding domains (reviewed by Chang et al., 2015a), and facilitate nuclear migration through actin TAN lines as opposed to microtubules in migrating cells (reviewed by Luxton et al., 2011). While our observations did not show the same pattern as TAN lines, it would be interesting to test the requirement of the nuclear envelope for this fast migration, as well as to investigate the localisation and function of different LINC complex proteins in mouse zygotes.

In order to further test the precise functions and interactions of Rab11a, Spire2, Formin-2 and actin, it would be interesting to investigate how these proteins are transiently recruited to the cortical protrusion overlying the forming pronucleus. As shown in this thesis, female DNA forming a pronucleus at the cortex was able to induce a protrusion of the cortex and the same enrichment of mClover3-Spire2 and mScarlet-Rab11a as observed adjacent to male pronuclei. The most obvious candidate for this recruitment is the Ran-GTP pathway. Ran GEFs localise to chromatin, activating Ran GTPases in a localised gradient, which in turn modify the surrounding cytoskeleton (Dumont et al., 2007b; Kalab et al., 1999). It is critical for mitotic (Kalab et al., 1999) and meiotic (Holubcová et al., 2015) spindle assembly in some species, as well as for the formation of actin-rich protrusions in mouse eggs through the Arp2/3 pathway (Deng et al., 2007; Yi et al., 2011). Paternal (or when induced to localise as such, maternal) DNA adjacent to the cortex could signal to the overlying cortex through the Ran GTPase pathway, inducing both the cortical protrusion and recruitment of Rab11a, Spire2, and Formin-2. The dominant-negative Ran ${ }^{T 24 N}$ could be used to test this, as it has previously been shown to perturb the Ran GTPase pathway in mouse and human oocytes (Baumann et al., 2017; Deng et al., 2007; Holubcová et al., 2015). One relevant observation reported in this thesis is that when fluorescently tagged Spire2 was expressed at very high levels, the described Spire2 'ring' had a much larger diameter surrounding the sperm entry site; the Spire2 accumulation that later constricted into a smaller area at the forming pronucleus started much further away from the sperm entry site than with lower expression levels (Figure 21). The centre of this ring, around which it constricted as the pronucleus formed, remained the sperm entry site, indicating that some signal must be originating from the DNA to coordinate Spire2 accumulation. It is unclear how overexpression of Spire2 would change its localisation if the RanGTP pathway was indeed signalling to the overlying cortex, as one would assume that the gradient of the Ran GEF would be unaffected by Spire2. In the future, it would be interesting to investigate this aspect in more detail, to find out more about the mechanisms of downstream effectors of RanGTP and how these can modulate protein localisation and vice versa, and to investigate other pathways that may be able to signal from the DNA to the surrounding cytoskeleton.

Although the focus of this thesis was early pronuclear migration, several aspects regarding the slower, microtubule- and actin-dependent pronuclear migration in the late zygote require further 
investigation in order to fully complement our new model of pronuclear migration in mouse zygotes. First, the origin of microtubule nucleation in the centriole-free mouse zygote would be of interest. As aMTOCs were not found to be required for this nucleation, it is possible that the Augmin-RanGTP pathway (reviewed by Meunier and Vernos, 2016) or recently described interphase-bridge (Zenker et al., 2017) could contribute to microtubule nucleation. The exact mechanisms of how microtubules facilitate late pronuclear migration would also be of interest. As Kathleen Scheffler's experiments implicated a role for dynein in this migration, the localisation of dynein and possible LINC complexes could be further investigated. In many cells, dynein facilitates directed migration along a polarised microtubule network, i.e. a network in which the minus ends face the direction of desired migration. As Kathleen Scheffler was not able to observe such a polarised microtubule network using different microtubule end markers, dynein may instead be facilitating microtubule crosslinking and sliding (Tanenbaum et al., 2013). Actin was required for microtubule-dependent centration of pronuclei, and it would also be interesting to find out which precise actin structures are important. We have shown that the Formin-2/Spire2-nucleated actin meshwork is dispensable for late pronuclear migration, so testing the function of other actin structures, such as the cortex, could yield further insight into this. While no perturbation has been described that targeted the actin cortex specifically, Arp2/3-mediated actin nucleation is important for subcortical thickening and control of cortical tension in mouse oocytes (Chaigne et al., 2013, 2015). Thus, the effect of Arp2/3 inhibition by CK666 (Hetrick et al., 2013) on pronuclear migration of mouse zygotes could be tested. Alternatively, it is possible that actin and microtubules interact directly. Such actin-microtubule crosstalk, mediated by proteins such as MAPs, plectin, and Tau, has been found to be critical for cell shape, morphogenesis, and other cellular functions in a range of contexts (reviewed by Dogterom and Koenderink, 2019; Mohan and John, 2015). Thus, investigating whether any of these proteins are required for the slower migration of pronuclei could be another future area of research.

In summary, our experiments have revealed a number of details regarding two pathways required for pronuclear migration in mouse zygotes, opening a vast array of future research questions. Importantly, our findings also have relevance outside of the specific context of mouse pronuclear migration.

\subsection{Significance of results in other contexts}

The centriole-free model system of the mouse zygote provides an interesting tool for investigation of alternative cytoskeletal mechanisms, including pronuclear migration, as discussed in this thesis, and spindle assembly (Courtois et al., 2012). Indeed, while pronuclear migration is largely actinindependent in most species (reviewed by Dupin and Etienne-Manneville, 2011 and in the 
introduction of this thesis), actin appears to be the critical cytoskeletal component required for both fast and slow pronuclear migration in mouse. As described in detail in the introduction, actin has been implicated in a variety of nuclear positioning events throughout development; through LINC complexes in wound healing (reviewed by Luxton et al., 2011), in collaboration with microtubules in C. elegans zygotes (Xiong et al., 2011), through actomyosin contractions in cortical interneurons (Martini and Valdeolmillos, 2010), or possibly directly as in the syncytial Drosophila embryo (Huelsmann et al., 2013). Although the exact mechanisms remain to be uncovered in future experiments, the microtubule- and actin-dependent slower migration of pronuclei in the later zygote may be somewhat similar to the situation in the $C$. elegans zygote, where microtubules nucleated by the sperm aster may push off the Arp2/3-dependent cell cortex to allow centration (Xiong et al., 2011). Rab11a, Spire2 and Formin-2 are required for GV centration (Almonacid et al., 2015) and meiotic spindle positioning (Holubcová et al., 2013) in mouse oocytes, but the transient accumulation of these proteins to the cortical region overlying the pronuclei appears to be a novel means of facilitating fast nuclear positioning. This raises a number of questions: How did this alternative mechanism evolve in rodents? Are the described mechanisms present in other centriole-free cells in mouse or other species? Even in centriole-containing systems, could actin be involved in nuclear positioning but disregarded due to the strong requirement for microtubules? Indeed, the continued investigation of nuclear positioning events throughout development in a wide range of species will be critical in defining the context of these new findings.

Nuclear positioning is critical in many clinical contexts beyond its relevance for basic biological research. Pronuclear migration specifically is critical for fertility, as its failure is detrimental to further embryonic development. In mouse embryos, failure of pronuclei to migrate completely to the centre resulted in multinucleation in the blastomeres of the two-cell-stage embryo (Reichmann et al., 2018). Nucleation status is important for further development, as incorrect chromatin localisation can lead to aneuploidy in later mitotic divisions. However, multinucleation itself may not always be detrimental to embryonic development; at the annual meeting of the European Society for Human Reproduction and Embryology (ESHRE), Marcos Meseguer Escriva from the IVIRMA Clinic in Valencia presented unpublished time-lapse images of human embryos able to correct multinucleation before blastocyst formation.

Human sperm contain centrioles and thus provide a sperm aster. As such, it is somewhat unlikely that the mechanisms described in this thesis are also important during human pronuclear migration; nonetheless, confirmation of this would be interesting. However, functional experiments with living human zygotes are limited by ethical and practical issues. Legally, scientific research using surplus human embryos from IVF treatments, or creating zygotes for a purpose other than fertility treatment, 
are strictly forbidden in Germany (Embryonenschutzgesetz (ESChG) §1). In countries where research on early human embryos is legal, surplus embryos are usually only discarded after developmental arrest during culture or at the blastocyst stage, long after pronuclear migration has taken place. Even if zygotes were donated to research, fertility treatment involves either conventional IVF, or ICSI. Conventional IVF requires overnight insemination, during which thousands of sperm are present in the medium, so that imaging of pronuclei at their formation would not be possible. During ICSI, the sperm is injected deep into the egg, so that the male pronucleus would not form at the cortex, preventing the described mechanisms from being tested (reviewed by Elder and Dale, 2011). Given that these experiments may thus be hard to achieve in human zygotes, further animal models must be considered. Mice have long been the standard mammalian model system, but oocytes and zygotes from other mammals such as pig and cow may provide a better system to investigate the clinical relevance of pronuclear migration mechanisms. Indeed, both pig (Kim et al., 1997) and cow (Navara et al., 1994; Payne et al., 2003) sperm have been shown to provide a sperm aster, and thus, a similar imaging approach applied to the zygotes of these animals could tell us whether the dynamics and mechanisms observed are conserved in other mammals. Importantly, while $\mathrm{Fmn}_{2}{ }^{-/}$mice are subfertile due to the requirement of Formin-2 for spindle migration in the oocyte, no mutations in FMN2 have been identified in IVF patients so far (Ryley et al., 2005), although this may be due to small sample size. Mechanisms of nuclear migration are also important for successful embryonic development, another feature relevant to fertility. As mentioned above, nuclear positioning is involved in the development of several organs and tissues in many species, and incorrect nuclear positioning may be a factor in a range of developmental defects and miscarriages, further contributing to fertility-related issues.

In addition to fertility, our findings may have relevance to other clinical areas in which nuclear positioning has been shown to be key, including muscular dystrophy, cardiomyopathy, lissencephaly, and cancer. Nuclei are usually positioned at the periphery of each myofiber cell, and centrallypositioned nuclei have been found in muscles of patients with two common dystrophies, Duchenne Muscular Dystrophy (Wang et al., 2000a) and Emery-Dreifuss Muscular Dystrophy (Gueneau et al., 2009). These muscular dystrophies are genetic in nature; Duchenne Muscular Dystrophy is typically caused by disruptions of the reading frame in the dystrophin gene (Koenig et al., 1989), while EmeryDreifuss Muscular Dystrophy is caused by a variety of genetic mutations. Interestingly, many of these mutations are found in nucleus-related genes rather than in muscle-specific genes (Brown et al., 2008). Mutations in LINC complex components Emerin (Bione et al., 1994), nesprins (Zhang et al., 2007), and lamins (Bonne et al., 1999) have been identified in patients with Emery-Dreifuss Muscular Dystrophy. While all of these findings implicate nuclear migration facilitated by LINC complexes as 
being important in muscular dystrophies, a direct link has not been investigated (reviewed by Folker and Baylies, 2013). Patients with lissencephaly type I have a smooth cerebral cortex, which can be caused by a mutation in the gene LIS1. LIS1 is important for nuclear migration in migrating neuronal precursors (Tsai et al., 2005), and neuronal migration is critical in the development of this disease (Reiner et al., 1993). It is thus possible that nuclear migration defects play a role in lissencephaly type I. Finally, the development of a variety of cancers has been associated with nuclear migration defects or mutations in LINC complexes, including severe epithelial dysplasia in colorectal adenomas (Konishi and Morson, 1982), ovarian and breast cancer (Marmé et al., 2008; Sjöblom et al., 2006), and colorectal cancers (Sjöblom et al., 2006). A causal link between nuclear positioning defects and these diseases has not been directly found, but the mechanisms whereby LINC complex defects, or mutations in other genes involved with nuclear positioning affect the development of a variety of diseases should be focus of future research (reviewed by Gundersen and Worman, 2013). Whether the nuclear positioning mechanisms described in this thesis are also relevant to any human diseases remains to be found. 


\subsection{Conclusions}

The results shown in this thesis, in combination with further work by Kathleen Scheffler, provide evidence for a new model of pronuclear migration in mouse zygotes. Using a novel protocol to image and track pronuclear migration in live zygotes, a variety of experiments revealed that Rab11a, Spire2 and Formin-2 are critical to allow fast migration of pronuclei away from the cortex in the early zygote, and a growing microtubule network takes over and centres the pronuclei in the later zygote. Several open questions remain, and progress in cell biological methods will hopefully allow for more detailed characterisation of the described mechanisms in the future. Furthermore, conservation of these mechanisms throughout evolution and their role in disease remains to be elucidated. 


\section{REFERENCES}

Agop-Nersesian, C., Naissant, B., Rached, F. Ben, Rauch, M., Kretzschmar, A., Thiberge, S., Menard, R., Ferguson, D.J.P., Meissner, M., and Langsley, G. (2009). Rab11A-controlled assembly of the inner membrane complex is required for completion of apicomplexan cytokinesis. PLoS Pathogens 5, e1000270.

Ajduk, A., llozue, T., Windsor, S., Yu, Y., Seres, K.B., Bomphrey, R.J., Tom, B.D., Swann, K., Thomas, A., Graham, C., et al. (2011). Rhythmic actomyosin-driven contractions induced by sperm entry predict mammalian embryo viability. Nature Communications 2:417.

Akhmanova, A., and Steinmetz, M.O. (2015). Control of microtubule organization and dynamics: Two ends in the limelight. Nature Reviews Molecular Cell Biology 16, 711-726.

Albertson, D.G. (1984). Formation of the first cleavage spindle in nematode embryos. Developmental Biology 101, 61-72.

Almonacid, M., Ahmed, W.W., Bussonnier, M., Mailly, P., Betz, T., Voituriez, R., Gov, N.S., and Verlhac, M.-H. (2015). Active diffusion positions the nucleus in mouse oocytes. Nature Cell Biology 17, 470479.

Almonacid, M., Terret, M.E., and Verlhac, M.H. (2018). Control of nucleus positioning in mouse oocytes. Seminars in Cell and Developmental Biology 82, 34-40.

Amorim, M.J., Bruce, E.A., Read, E.K.C., Foeglein, A., Mahen, R., Stuart, A.D., and Digard, P. (2011). A Rab11- and Microtubule-Dependent Mechanism for Cytoplasmic Transport of Influenza A Virus Viral RNA. Journal of Virology 85, 4143-4156.

Aoki, F., Worrad, D.M., and Schultz, R.M. (1997). Regulation of transcriptional activity during the first and second cell cycles in the preimplantation mouse embryo. Developmental Biology 181, 296-307.

Baumann, C., Wang, X., Yang, L., and Viveiros, M.M. (2017). Error-prone meiotic division and subfertility in mice with oocyte-conditional knockdown of pericentrin. Journal of Cell Science 130, $1251-1262$.

Bedford, J.M. (1981). Why mammalian gametes don't mix. Nature 291, 286-288.

Belin, B.J., Lee, T., and Mullins, R.D. (2015). DNA damage induces nuclear actin filament assembly by Formin-2 and Spire-1/2 that promotes efficient DNA repair. ELife 4, e07735.

Bellion, A., Baudoin, J.-P., Alvarez, C., Bornens, M., and Metin, C. (2005). Nucleokinesis in Tangentially 
Migrating Neurons Comprises Two Alternating Phases: Forward Migration of the Golgi/Centrosome Associated with Centrosome Splitting and Myosin Contraction at the Rear. The Journal of Neuroscience 25, 5691-5699.

Benedetti, L., Barentine, A.E.S., Messa, M., Wheeler, H., Bewersdorf, J., and De Camilli, P. (2018). Lightactivated protein interaction with high spatial subcellular confinement. Proceedings of the National Academy of Sciences 115, E2238-E2245.

Bestor, T.H., and Schatten, G. (1981). Anti-tubulin Immunofluorescence Microscopy of Microtubules Present during the Pronuclear Movements of Sea Urchin Fertilization. Developmental Biology 88, 8091.

Bhuin, T., and Roy, J.K. (2011). Rab11 is required for cell adhesion, maintenance of cell shape and actin-cytoskeleton organization during Drosophila wing development. International Journal of Developmental Biology 55, 269-279.

Bianchi, E., and Wright, G.J. (2016). Sperm Meets Egg: The Genetics of Mammalian Fertilization. Annual Review of Genetics 50, 93-111.

Bianchi, E., Doe, B., Goulding, D., and Project, S.M.G. (2014). Juno is the egg Izumo receptor and is essential for mammalian fertilisation. Nature 508, 483-487.

Bione, S., Maestrini, E., Rivella, S., Mancini, M., Regis, S., Romeo, G., and Toniolo, D. (1994). Identification of a novel X-linked gene responsible for Emery- Dreifuss muscular dystrophy. Nature Genetics 8, 323-327.

Blanchoin, L., Boujemaa-Paterski, R., Sykes, C., and Plastino, J. (2014). Actin Dynamics, Architecture, and Mechanics in Cell Motility. Physiological Reviews 94, 235-263.

Bleil, J.D., and Wassarman, P.M. (1980). Structure and Function of the Zona Pellucida: Identification and Characterization of the Proteins of the Mouse Oocyte's Zona Pellucida. Developmental Biology 76, 185-202.

Bleil, J.D., Beall, C.F., and Wassarman, P.M. (1981). Mammalian sperm-egg interaction: Fertilization of mouse eggs triggers modification of the major zona pellucida glycoprotein, ZP2. Developmental Biology 86, 189-197.

Bone, C.R., and Starr, D.A. (2016). Nuclear migration events throughout development. Journal of Cell Science 129, 1951-1961.

Bonne, G., Di Barletta, M.R., Varnous, S., Bécane, H.-M., Hammouda, E.-H., Merlini, L., Muntoni, F., 
Greenberg, C.R., Gary, F., Urtizberea, J.-A., et al. (1999). Mutations in the gene encoding lamin A/C cause autosomal dominant Emery-Dreifuss muscular dystrophy. Nature Genetics 21, 285-288.

Borrego-Pinto, J., Jegou, T., Osorio, D.S., Aurade, F., Gorjanacz, M., Koch, B., Mattaj, I.W., and Gomes, E.R. (2012). Samp1 is a component of TAN lines and is required for nuclear movement. Journal of Cell Science 125, 1099-1105.

Borum, K. (1961). Oogenesis in the Mouse: A Study of the Meiotic Prophase. Experimental Cell Research 24, 495-507.

Bos-Mikich, A., Swann, K., and Whittingham, D.G. (1995). Calcium Oscillations and Protein Synthesis Inhibition Synergistically Activate Mouse Oocytes. Molecular Reproduction and Development 41, 8490.

Bosch, M., Le, K.H.D., Bugyi, B., Correia, J.J., Renault, L., and Carlier, M.F. (2007). Analysis of the Function of Spire in Actin Assembly and Its Synergy with Formin and Profilin. Molecular Cell 28, 555568.

Bouchet, J., del Río-Iñiguez, I., Lasserre, R., Agüera-Gonzalez, S., Cuche, C., Danckaert, A., McCaffrey, M.W., Di Bartolo, V., and Alcover, A. (2016). Rac1-Rab11-FIP3 regulatory hub coordinates vesicle traffic with actin remodeling and T-cell activation. The EMBO Journal 35, 1160-1174.

Bouniol-Baly, C., Hamraoui, L., Guibert, J., Beaujean, N., Szöllösi, M.S., and Debey, P. (1999). Differential Transcriptional Activity Associated with Chromatin Configuration in Fully Grown Mouse Germinal Vesicle Oocytes1. Biology of Reproduction 60, 580-587.

Brouhard, G.J., and Rice, L.M. (2018). Microtubule dynamics: An interplay of biochemistry and mechanics. Nature Reviews Molecular Cell Biology 19, 451-463.

Brown, S.C., Piercy, R.J., Muntoni, F., and Sewry, C.A. (2008). Investigating the pathology of EmeryDreifuss muscular dystrophy. Biochemical Society Transactions 36, 1335-1338.

Brunet, S., and Maro, B. (2007). Germinal vesicle position and meiotic maturation in mouse oocyte. Reproduction 133, 1069-1072.

Brunet, S., and Verlhac, M.H. (2011). Positioning to get out of meiosis: the asymmetry of division. Human Reproduction Update 17, 68-75.

Bultman, S.J., Gebuhr, T.C., Pan, H., Svoboda, P., Schultz, R.M., and Magnuson, T. (2006). Maternal BRG1 regulates zygotic genome activation in the mouse. Genes and Development 20, 1744-1754. 
Bun, P., Dmitrieff, S., Belmonte, J.M., Nédélec, F.J., and Lénárt, P. (2018). A disassembly-driven mechanism explains F-actin-mediated chromosome transport in starfish oocytes. ELife 7, e31469.

Burkart, A.D., Xiong, B., Baibakov, B., Jiménez-Movilla, M., and Dean, J. (2012). Ovastacin, a cortical granule protease, cleaves ZP2 in the zona pellucida to prevent polyspermy. Journal of Cell Biology 197, 37-44.

Chaigne, A., Campillo, C., Gov, N.S., Voituriez, R., Azoury, J., Umaña-Diaz, C., Almonacid, M., Queguiner, I., Nassoy, P., Sykes, C., et al. (2013). A soft cortex is essential for asymmetric spindle positioning in mouse oocytes. Nature Cell Biology 15, 958-966.

Chaigne, A., Campillo, C., Gov, N.S., Voituriez, R., Sykes, C., Verlhac, M.H., and Terret, M.E. (2015). A narrow window of cortical tension guides asymmetric spindle positioning in the mouse oocyte. Nature Communications 6, 6027.

Chaigne, A., Campillo, C., Voituriez, R., Gov, N.S., Sykes, C., Verlhac, M.-H., and Terret, M.-E. (2016). Factin mechanics control spindle centring in the mouse zygote. Nature Communications 7, 10253.

Chalbi, M., Barraud-Lange, V., Ravaux, B., Howan, K., Rodriguez, N., Soule, P., Ndzoudi, A., Boucheix, C., Rubinstein, E., Wolf, J.P., et al. (2014). Binding of sperm protein Izumo1 and its egg receptor Juno drives Cd9 accumulation in the intercellular contact area prior to fusion during mammalian fertilization. Development 141, 3732-3739.

Chambers, E.L. (1939). The movement of the egg nucleus in relation to the sperm aster in the echinoderm egg. Journal of Experimental Biology 16, 409-424.

Chang, W., Folker, E.S., Worman, H.J., and Gundersen, G.G. (2013). Emerin organizes actin flow for nuclear movement and centrosome orientation in migrating fibroblasts. Molecular Biology of the Cell 24, 3869-3880.

Chang, W., Worman, H.J., and Gundersen, G.G. (2015a). Accessorizing and anchoring the LINC complex for multifunctionality. Journal of Cell Biology 208, 11-22.

Chang, W., Antoku, S., Östlund, C., Worman, H.J., and Gundersen, G.G. (2015b). Linker of nucleoskeleton and cytoskeleton (LINC) complex-mediated actin-dependent nuclear positioning orients centrosomes in migrating myoblasts. Nucleus $6,77-88$.

Chang, W., Antoku, S., and Gundersen, G. (2016). Wound-Healing Assays to Study Mechanisms of Nuclear Movement in Fibroblasts and Myoblasts. In The Nuclear Envelope: Methods and Protocols, S. Shakleton, P. Collas, and E. Schirmer, eds. (New York: Humana Press), pp. 255-267. 
Cheeseman, L.P., Boulanger, J., Bond, L.M., and Schuh, M. (2016). Two pathways regulate cortical granule translocation to prevent polyspermy in mouse oocytes. Nature Communications 7, 13726.

Chesarone, M.A., DuPage, A.G., and Goode, B.L. (2010). Unleashing formins to remodel the actin and microtubule cytoskeletons. Nature Reviews Molecular Cell Biology 11, 62-74.

Chew, T.G., Lorthongpanich, C., Ang, W.X., Knowles, B.B., and Solter, D. (2012). Symmetric Cell Division of the Mouse Zygote Requires an Actin Network. Cytoskeleton 69, 1040-1046.

Clift, D., and Schuh, M. (2013). Restarting life: fertilization and the transition from meiosis to mitosis. Nature Reviews Molecular Cell Biology 14, 549-562.

Clift, D., and Schuh, M. (2015). A three-step MTOC fragmentation mechanism facilitates bipolar spindle assembly in mouse oocytes. Nature Communications 6, 7217.

Clift, D., McEwan, W.A., Labzin, L.I., Konieczny, V., Mogessie, B., James, L.C., and Schuh, M. (2018). A Method for the Acute and Rapid Degradation of Endogenous Proteins. Cell 172, 1-15.

Cockell, M.M., Baumer, K., and Gönczy, P. (2004). lis-1 is required for dynein-dependent cell division processes in C. elegans embryos. Journal of Cell Science 117, 4571-4582.

Cooley, L., Verheyen, E., and Ayers, K. (1992). chickadee Encodes a Profilin Required for Intercellular Cytoplasm Transport during Drosophila Oogenesis. Cell 69, 173-184.

Courtois, A., Schuh, M., Ellenberg, J., and Hiiragi, T. (2012). The transition from meiotic to mitotic spindle assembly is gradual during early mammalian development. Journal of Cell Biology 198, 357370.

Dahlgaard, K., Raposo, A.A.S.F., Niccoli, T., and St Johnston, D. (2007). Capu and Spire Assemble a Cytoplasmic Actin Mesh that Maintains Microtubule Organization in the Drosophila Oocyte. Developmental Cell 13, 539-553.

Decker, F., and Brugués, J. (2015). Dissecting microtubule structures by laser ablation. Methods in Cell Biology 125, 61-75.

Deguchi, R., Shirakawa, H., Oda, S., Mohri, T., and Miyazaki, S. (2000). Spatiotemporal analysis of Ca2+ waves in relation to the sperm entry site and animal-vegetal axis during Ca2+oscillations in fertilized mouse eggs. Developmental Biology 218, 299-313.

Deng, M.-Q., and Shen, S.S. (2000). A Specific Inhibitor of p34cdc2/Cyclin B Suppresses FertilizationInduced Calcium Oscillations in Mouse Eggs. Biology of Reproduction 62, 873-878. 
Deng, M., and Li, R. (2009). Sperm chromatin-induced ectopic polar body extrusion in mouse eggs after ICSI and delayed egg activation. PLoS ONE 4, e7171.

Deng, M., Kishikawa, H., Yanagimachi, R., Kopf, G.S., Schultz, R.M., and Williams, C.J. (2003). Chromatin-mediated cortical granule redistribution is responsible for the formation of the cortical granule-free domain in mouse eggs. Developmental Biology 257, 166-176.

Deng, M., Williams, C.J., and Schultz, R.M. (2005). Role of MAP kinase and myosin light chain kinase in chromosome-induced development of mouse egg polarity. Developmental Biology 278, 358-366.

Deng, M., Suraneni, P., Schultz, R.M., and Li, R. (2007). The Ran GTPase Mediates Chromatin Signaling to Control Cortical Polarity during Polar Body Extrusion in Mouse Oocytes. Developmental Cell 12, 301-308.

Dogterom, M., and Koenderink, G.H. (2019). Actin-microtubule crosstalk in cell biology. Nature Reviews Molecular Cell Biology 20, 38-54.

Drummond, D.R., and Cross, R.A. (2000). Dynamics of interphase microtubules in Schizosaccharomyces pombe. Current Biology 10, 766-775.

Ducka, A.M., Joel, P., Popowicz, G.M., Trybus, K.M., Schleicher, M., Noegel, A.A., Huber, R., Holak, T.A., and Sitar, T. (2010). Structures of actin-bound Wiskott-Aldrich syndrome protein homology 2 (WH2) domains of Spire and the implication for filament nucleation. Proceedings of the National Academy of Sciences 107, 11757-11762.

Dumont, J., Million, K., Sunderland, K., Rassinier, P., Lim, H., Leader, B., and Verlhac, M. (2007a). Formin-2 is required for spindle migration and for the late steps of cytokinesis in mouse oocytes. Developmental Biology 301, 254-265.

Dumont, J., Petri, S., Pellegrin, F., Terret, M.E., Bohnsack, M.T., Rassinier, P., Georget, V., Kalab, P., Gruss, O.J., and Verlhac, M.H. (2007b). A centriole- and RanGTP-independent spindle assembly pathway in meiosis I of vertebrate oocytes. The Journal of Cell Biology 176, 295-305.

Dupin, I., and Etienne-Manneville, S. (2011). Nuclear positioning: Mechanisms and functions. International Journal of Biochemistry and Cell Biology 43, 1698-1707.

Eisfeld, A.J., Kawakami, E., Watanabe, T., Neumann, G., and Kawaoka, Y. (2011). RAB11A Is Essential for Transport of the Influenza Virus Genome to the Plasma Membrane. Journal of Virology 85, 61176126.

Elder, K., and Dale, B. (2011). In-Vitro Fertilization (Cambridge, England: Cambridge University Press). 
Emmons, S., Phan, H., Calley, J., Chen, W., and James, B. (1995). cappuccino, a Drosophila maternal effect gene required for polarity of the egg and embryo, is related to the vertebrate limb deforminty locus. Genes \& Development 2482-2494.

Fan, S.-S., and Ready, D.F. (1997). Glued participates in distinct microtubule-based activities in Drosophila eye development. Development 124, 1497-1507.

Firat-Karalar, E.N., and Welch, M.D. (2011). New mechanisms and functions of actin nucleation. Current Opinion in Cell Biology 23, 4-13.

Fischer-Vize, J.A., and Mosley, K.L. (1994). marbles mutants: uncoupling cell determination and nuclear migration in the developing Drosophila eye. Development 120, 2609-2618.

Folker, E.S., and Baylies, M.K. (2013). Nuclear positioning in muscle development and disease. Frontiers in Physiology 4, 363.

Folker, E.S., Ostlund, C., Luxton, G.W.G., Worman, H.J., and Gundersen, G.G. (2011). Lamin A variants that cause striated muscle disease are defective in anchoring transmembrane actin-associated nuclear lines for nuclear movement. Proceedings of the National Academy of Sciences 108, 131-136.

Fridolfsson, H.N., Ly, N., Meyerzon, M., and Starr, D.A. (2010). UNC-83 coordinates kinesin-1 and dynein activities at the nuclear envelope during nuclear migration. Developmental Biology 338, 237250.

Gibson, D.G., Young, L., Chuang, R.-Y., Venter, J.C., Hutchison, C.A., and Smith, H.O. (2009). Enzymatic assembly of DNA molecules up to several hundred kilobases. Nature Methods 6, 343-345.

Gomes, E.R., Jani, S., and Gundersen, G.G. (2005). Nuclear Movement Regulated by Cdc42, MRCK, Myosin, and Actin Flow Establishes MTOC Polarization in Migrating Cells. Cell 121, 451-463.

Gönczy, P., Pichler, S., Kirkham, M., and Hyman, A.A. (1999). Cytoplasmic Dynein Is Required for Distinct Aspects of MTOC Positioning, Including Centrosome Separation, in the One Cell Stage Caenorhabditis elegans Embryo. The Journal of Cell Biology 147, 135-150.

Goodson, H. V, and Jonasson, E.M. (2018). Microtubules and Microtubule-Associated Proteins. Cold Spring Harbor Perspectives in Biology 10, a022608.

Goulding, M.B., Canman, J.C., Senning, E.N., Marcus, A.H., and Bowerman, B. (2007). Control of nuclear centration in the C. elegans zygote by receptor-independent Galpha signaling and myosin II. The Journal of Cell Biology 178, 1177-1191. 
Green, M.R., and Sambrook, J. (2012). Molecular Cloning: A Laboratory Manual (Cold Spring Harbor Laboratory Press).

Griswold, M.D. (2016). Spermatogenesis: The Commitment to Meiosis. Physiological Reviews 96, 117.

Gu, T.P., Guo, F., Yang, H., Wu, H.P., Xu, G.F., Liu, W., Xie, Z.G., Shi, L., He, X., Jin, S.G., et al. (2011). The role of Tet3 DNA dioxygenase in epigenetic reprogramming by oocytes. Nature 477, 606-612.

Gueneau, L., Bertrand, A.T., Jais, J.P., Salih, M.A., Stojkovic, T., Wehnert, M., Hoeltzenbein, M., Spuler, S., Saitoh, S., Verschueren, A., et al. (2009). Mutations of the FHL1 Gene Cause Emery-Dreifuss Muscular Dystrophy. American Journal of Human Genetics 85, 338-353.

Guild, G.M., Connelly, P.S., Shaw, M.K., and Tilney, L.G. (1997). Actin Filament Cables in Drosophila Nurse Cells Are Composed of Modules That Slide Passively Past One Another during Dumping. The Journal of Cell Biology 138, 783-797.

Gundersen, G.G., and Worman, H.J. (2013). Nuclear positioning. Cell 152, 1376-1389.

Guntas, G., Hallett, R.A., Zimmerman, S.P., Williams, T., Yumerefendi, H., Bear, J.E., and Kuhlman, B. (2015). Engineering an improved light-induced dimer (iLID) for controlling the localization and activity of signaling proteins. Proceedings of the National Academy of Sciences of the United States of America $112,112-117$.

Hamaguchi, M.S., and Hiramoto, Y. (1986). Analysis of the role of astral rays in pronuclear migration in sand dollar eggs by the coldemid-UV method. Development Growth \& Differentiation 28, 143-156.

Hansen, D. V., Tung, J.J., and Jackson, P.K. (2006). CaMKII and Polo-like kinase 1 sequentially phosphorylate the cytostatic factor Emi2/XErp1 to trigger its destruction and meiotic exit. Proceedings of the National Academy of Sciences 103, 608-613.

Hartman, M.A., and Spudich, J.A. (2012). The myosin superfamily at a glance. Journal of Cell Science $125,1627-1632$.

Hetrick, B., Han, M.S., Helgeson, L.A., and Nolen, B.J. (2013). Small molecules CK-666 and CK-869 inhibit Arp2/3 complex by blocking an activating conformational change. Chemical Biology 20, 701712.

Hird, S.N., and White, J.G. (1993). Cortical and cytoplasmic flow polarity in early embryonic cells of Caenorhabditis elegans. The Journal of Cell Biology 121, 1343-1355. 
Holubcová, Z., Howard, G., and Schuh, M. (2013). Vesicles modulate an actin network for asymmetric spindle positioning. Nature Cell Biology 15, 937-947.

Holubcová, Z., Blayney, M., Elder, K., and Schuh, M. (2015). Human oocytes. Error-prone chromosomemediated spindle assembly favors chromosome segregation defects in human oocytes. Science 348, $1143-1147$.

Hörandl, E. (2013). Meiosis and the Paradox of Sex in Nature. In Biochemistry, Genetics and Molecular Biology: Meiosis, pp. 17-39.

Hoyer-Fender, S. (2012). Centrosomes in fertilization, early embryonic development, stem cell division, and cancer. Atlas of Genetics and Cytogenetics in Oncology and Haematology 16, 306-319.

Huang, L., Meng, T.G., Ma, X.S., Wang, Z.B., Qi, S.T., Chen, Q., Zhang, Q.H., Liang, Q.X., Wang, Z.W., Hu, M.W., et al. (2019). Rad9a is involved in chromatin decondensation and post-zygotic embryo development in mice. Cell Death and Differentiation 26, 969-980.

Huelsmann, S., Ylänne, J., and Brown, N.H. (2013). Filopodia-like actin cables position nuclei in association with perinuclear actin in Drosophila nurse cells. Developmental Cell 26, 604-615.

Inoue, N., Ikawa, M., Isotani, A., and Okabe, M. (2005). The Immunoglobulin Superfamily Protein Izumo is Required for Sperm to Fuse with Eggs. Nature 434, 234-238.

Ito, S., Okuda, S., Abe, M., Fujimoto, M., Onuki, T., Nishimura, T., and Takeichi, M. (2017). Induced cortical tension restores functional junctions in adhesion-defective carcinoma cells. Nature Communications 8, 1834.

Jackson, C.L., and Casanova, J.E. (2000). Turning on ARF: The Sec7 family of guanine-nucleotideexchange factors. Trends in Cell Biology 10, 60-67.

Jaffe, L.A., and Terasaki, M. (2004). Quantitative Microinjection of Oocytes, Eggs, and Embryos. Methods in Cell Biology 74, 219-242.

Jayo, A., Malboubi, M., Antoku, S., Chang, W., Ortiz-Zapater, E., Groen, C., Pfisterer, K., Tootle, T., Charras, G., Gundersen, G.G., et al. (2016). Fascin Regulates Nuclear Movement and Deformation in Migrating Cells. Developmental Cell 38, 371-383.

Jegou, A., Ziyyat, A., Barraud-Lange, V., Perez, E., Wolf, J.P., Pincet, F., and Gourier, C. (2011). CD9 tetraspanin generates fusion competent sites on the egg membrane for mammalian fertilization. Proceedings of the National Academy of Sciences 108, 10946-10951. 
Ji, H.-H., Yao, L.-L., Liu, C., and Li, X. (2019). Regulation of Myosin-5b by Rab11a and the Rab11 family interacting protein 2. Bioscience Reports 39, BSR20181252.

Jones, K.T., Carroll, J., Merriman, J. a, Whittingham, D.G., and Kono, T. (1995). Repetitive sperminduced $\mathrm{Ca} 2+$ transients in mouse oocytes are cell cycle dependent. Development (Cambridge, England) 121, 3259-3266.

Kalab, P., Pu, R.T., and Dasso, M. (1999). The Ran GTPase regulates mitotic spindle assembly. Current Biology 9, 481-484.

Kim, N.H., Chung, K.S., and Day, B.N. (1997). The distribution and requirements of microtubules and microfilaments during fertilization and parthenogenesis in pig oocytes. Reproduction 111, 143-149.

Kimura, A., and Onami, S. (2005). Computer simulations and image processing reveal lengthdependent pulling force as the primary mechanism for $\mathrm{C}$. elegans male pronuclear migration. Developmental Cell 8, 765-775.

Kimura, K., and Kimura, A. (2011). Intracellular organelles mediate cytoplasmic pulling force for centrosome centration in the Caenorhabditis elegans early embryo. Proceedings of the National Academy of Sciences 108, 137-142.

Kimura, M., Kim, E., Kang, W., Yamashita, M., Saigo, M., Yamazaki, T., Nakanishi, T., Kashiwabara, S., and Baba, T. (2009). Functional Roles of Mouse Sperm Hyaluronidases, HYAL5 and SPAM1, in Fertilization1. Biology of Reproduction 81, 939-947.

Kobayashi, H., Sakurai, T., Imai, M., Takahashi, N., Fukuda, A., Yayoi, O., Sato, S., Nakabayashi, K., Hata, K., Sotomaru, Y., et al. (2012). Contribution of intragenic DNA methylation in mouse gametic DNA methylomes to establish Oocyte-specific heritable marks. PLoS Genetics 8.

Koenig, M., Beggs, A.H., Moyer, M., Scherpf, S., Heindrich, K., Bettecken, T., Meng, G., Müller, C.R., Lindlöf, M., Kaariainen, H., et al. (1989). The Molecular Basis for Duchenne versus Becker Muscular Dystrophy: Correlation of Severity with Type of Deletion. American Journal of Human Genetics 45, 498-506.

Konishi, F., and Morson, B.C. (1982). Pathology of colorectal adenomas: A colonoscopic survey. Journal of Clinical Pathology 35, 830-841.

Kracklauer, M.P., Banks, S.M.L., Xie, X., Wu, Y., and Fischer, J.A. (2007). Drosophila klaroid encodes a SUN domain protein required for klarsicht localization to the nuclear envelope and nuclear migration in the eye. Fly $1,75-85$. 
Kutscheidt, S., Zhu, R., Antoku, S., Luxton, G., Stagljar, I., Fackler, O., and Gundersen, G. (2014). FHOD1 interaction with nesprin-2G mediates TAN line formation and nuclear movement. Nature Cell Biology $16,708-715$.

Kyozuka, K., Chun, J.T., Puppo, A., Gragnaniello, G., Garante, E., and Santella, L. (2008). Actin cytoskeleton modulates calcium signaling during maturation of starfish oocytes. Developmental Biology 320, 426-435.

Lapierre, L.A., Dorn, M.C., Zimmerman, C.F., Navarre, J., Burnette, J.O., and Goldenring, J.R. (2003). Rab11b resides in a vesicular compartment distinct from Rab11a in parietal cells and other epithelial cells. Experimental Cell Research 290, 322-331.

Larose, H., Shami, A.N., Abbott, H., Manske, G., Lei, L., and Hammoud, S.S. (2019). Gametogenesis: A journey from inception to conception (Elsevier Inc.).

Larson, S.M., Lee, H.J., Hung, P., Matthrews, L.M., Robinson, D., and Evans, J. (2010). Cortical Mechanics and Meiosis II Completion in Mammalian Oocytes Are Mediated by Myosin-II and EzrinRadixin-Moesin (ERM) Proteins. Molecular Biology of the Cell 21, 3182-3192.

Leader, B., and Leder, P. (2000). Formin-2, a novel formin homology protein of the cappuccino subfamily, is highly expressed in the developing and adult central nervous system. Mechanisms of Development 93, 221-231.

Leader, B., Lim, H., Carabatsos, M.J., Harrington, A., Ecsedy, J., Pellman, D., Maas, R., and Leder, P. (2002). Formin-2, polyploidy, hypofertility and positioning of the meiotic spindle in mouse oocytes. Nature Cell Biology 4, 921-928.

Lee, Y.L., and Burke, B. (2018). LINC complexes and nuclear positioning. Seminars in Cell and Developmental Biology 82, 67-76.

Lefièvre, L., Conner, S.J., Salpekar, A., Olufowobi, O., Ashton, P., Pavlovic, B., Lenton, W., Afnan, M., Brewis, I.A., Monk, M., et al. (2004). Four zona pellucida glycoproteins are expressed in the human. Human Reproduction 19, 1580-1586.

Lele, T.P., Dickinson, R.B., and Gundersen, G.G. (2018). Mechanical principles of nuclear shaping and positioning. The Journal of Cell Biology 217, 3330-3342.

Li, H., Guo, F., Rubinstein, B., and Li, R. (2008). Actin-driven chromosomal motility leads to symmetry breaking in mammalian meiotic oocytes. Nature Cell Biology 10, 1301-1308.

Liman, E.R., Tytgat, J., and Hess, P. (1992). Subunit stoichiometry of a mammalian K+ channel 
determined by construction of multimeric cDNAs. Neuron 9, 861-871.

Lin, C.-J., Koh, F.M., Wong, P., Conti, M., and Ramalho-Santos, M. (2014). Hira-mediated H3.3 incorporation is required for DNA replication and ribosomal RNA transcription in the mouse zygote. Developmental Cell 30, 268-279.

Lindeman, R.E., and Pelegri, F. (2012). Localized products of futile cycle/Irmp promote centrosomenucleus attachment in the zebrafish zygote. Current Biology 22, 843-851.

Lomakin, A.J., Lee, K.-C., Han, S.J., Bui, D.A., Davidson, M., Mogilner, A., and Danuser, G. (2015). Competition of two distinct actin networks for actin defines a bistable switch for cell polarization HHS Public Access. Nature Cell Biology 17, 1435-1445.

Loppin, B., Dubruille, R., and Horard, B. (2015). The intimate genetics of Drosophila fertilization. Open Biology 5.

Luxton, G., Gomes, E., Folker, E., Vintinner, E., and Gundersen, G. (2010). Linear Arrays of Nuclear Envelope Proteins Harness Retrograde Actin Flow for Nuclear Movement. Science 329, 956-959.

Luxton, G., Gomes, E.R., Folker, E.S., Worman, H.J., and Gundersen, G.G. (2011). TAN lines: A novel nuclear envelope structure involved in nuclear positioning. Nucleus 2, 173-181.

Ma, S.F., Liu, X.Y., Miao, D.Q., Han, Z. Bin, Zhang, X., Miao, Y.L., Yanagimachi, R., and Tan, J.H. (2005). Parthenogenetic activation of mouse oocytes by strontium chloride: A search for the best conditions. Theriogenology 64, 1142-1157.

MacLennan, M., Crichton, J.H., Playfoot, C.J., and Adams, I.R. (2015). Oocyte development, meiosis and aneuploidy. Seminars in Cell and Developmental Biology 45, 68-76.

Madgwick, S., Hansen, D. V., Levasseur, M., Jackson, P.K., and Jones, K.T. (2006). Mouse Emi2 is required to enter meiosis II by reestablishing cyclin B1 during interkinesis. The Journal of Cell Biology $174,791-801$.

Malone, C.J., Misner, L., Bot, N. Le, Tsai, M., Campbell, J.M., Ahringer, J., and White, J.G. (2003). The C. elegans Hook Protein, ZYG-12, Mediates the Essential Attachment between the Centrosome and Nucleus. Cell 115, 825-836.

Mana-Capelli, S., McLean, J.R., Chen, C.-T., Gould, K.L., and McCollum, D. (2012). The kinesin-14 Klp2 is negatively regulated by the SIN for proper spindle elongation and telophase nuclear positioning. Molecular Biology of the Cell 23, 4592-4600. 
Manandhar, G., Schatten, H., and Sutovsky, P. (2005). Centrosome Reduction During Gametogenesis and Its Significance1. Biology of Reproduction 72, 2-13.

Manseau, L.J., and Schüpbach, T. (1989). cappuccino and spire: two unique maternal-effect loci required for both the anteroposterior and dorsoventral patterns of the Drosophila embryo. Genes \& Development 3, 1437-1452.

Marmé, A., Zimmermann, H.P., Moldenhauer, G., Schorpp-Kistner, M., Müller, C., Keberlein, O., Giersch, A., Kretschmer, J., Seib, B., Spiess, E., et al. (2008). Loss of Drop1 expression already at early tumor stages in a wide range of human carcinomas. International Journal of Cancer 123, 2048-2056.

Maro, B., Johnson, M.H., Pickering, S.J., and Flach, A.G. (1984). Changes in actin distribution during fertilization of the mouse egg. Journal of Embryology and Experimental Morphology 81, 211-237.

Martini, F.J., and Valdeolmillos, M. (2010). Actomyosin Contraction at the Cell Rear Drives Nuclear Translocation in Migrating Cortical Interneurons. The Journal of Neuroscience 30, 8660-8670.

Matson, S., Markoulaki, S., and Ducibella, T. (2006). Antagonists of Myosin Light Chain Kinase and of Myosin II Inhibit Specific Events of Egg Activation in Fertilized Mouse Eggs1. Biology of Reproduction 74, 169-176.

Mayer, W., Niveleau, A., Walter, J., Fundele, R., and Haaf, T. (2000). Demethylation of the zygotic paternal genome. Nature 403, 501-502.

McNally, F.J. (2013). Mechanisms of spindle positioning. The Journal of Cell Biology 200, 131-140.

Meunier, S., and Vernos, I. (2016). Acentrosomal Microtubule Assembly in Mitosis: The Where, When, and How. Trends in Cell Biology 26, 80-87.

Miao, Y., Ma, S., Liu, X., Miao, D., Chang, Z., Luo, M., and Tan, J. (2004). Fate of the first polar bodies in mouse oocytes. Molecular Reproduction and Development 69, 66-76.

Miao, Y., Stein, P., Jefferson, W.N., Padilla-Banks, E., and Williams, C.J. (2012). Calcium influx-mediated signaling is required for complete mouse egg activation. Proceedings of the National Academy of Sciences 109, 4169-4174.

Miyado, K., Yoshida, K., Yamagata, K., Sakakibara, K., Okabe, M., Wang, X., Miyamoto, K., Akutsu, H., Kondo, T., Takahashi, Y., et al. (2008). The fusing ability of sperm is bestowed by CD9-containing vesicles released from eggs in mice. Proceedings of the National Academy of Sciences 105, 1292112926. 
Miyazaki, S., Yuzaki, M., Nakada, K., Shirakawa, H., Nakanishi, S., and Nakade, S. (1992). Block of Ca2+ Wave and Ca2+ Oscillation by Antibody to the Inositol 1,4,5-Trisphosphate Receptor in Fertilizes Hamster Eggs. Science 257, 251-255.

Miyazaki, S., Shirakawa, H., Nakada, K., and Honda, Y. (1993). Essential role of the inositol 1,4,5trisphosphate receptor/Ca2+ release channel in $\mathrm{Ca} 2+$ waves and $\mathrm{Ca} 2+$ oscillations at fertilization of mammalian eggs. Developmental Biology 158, 62-78.

Mogessie, B., Scheffler, K., and Schuh, M. (2018). Assembly and Positioning of the Oocyte Meiotic Spindle. Annual Review of Cell and Developmental Biology 34, 381-403.

Mohan, R., and John, A. (2015). Microtubule-associated proteins as direct crosslinkers of actin filaments and microtubules. International Union of Biochemistry and Molecular Biology 67, 395-403.

Montaville, P., Jegou, A., Pernier, J., Compper, C., Berengere, G., Mogessie, B., Schuh, M., RometLemonne, G., and Carlier, M.-F. (2014). Spire and Formin 2 Synergize and Antagonize in Regulating Actin Assembly in Meiosis by a Ping-Pong Mechanism. PLoS Biology 12, e1001795.

Mosley-Bishop, K.L., Li, Q., Patterson, K., and Fischer, J.A. (1999). Molecular analysis of the klarsicht gene and its role in nuclear migration within differentiating cells of the Drosophila eye. Current Biology 9, 1211-1220.

Nakamura, T., Liu, Y.J., Nakashima, H., Umehara, H., Inoue, K., Matoba, S., Tachibana, M., Ogura, A., Shinkai, Y., and Nakano, T. (2012). PGC7 binds histone H3K9me2 to protect against conversion of $5 \mathrm{mC}$ to $5 \mathrm{hmC}$ in early embryos. Nature $486,415-419$.

Navara, C.S., First, N.L., and Schatten, G. (1994). Microtubule Organization in the Cow during Fertilization, Polyspermy, Parthenogenesis, and Nuclear Transfer: The Role of the Sperm Aster. Developmental Biology 162, 29-40.

Nebenfuhr, A., Ritzenthaler, C., and Robinson, D.G. (2002). Brefeldin A: Deciphering an Enigmatic Inhibitor of Secretion. Plant Physiology 130, 1102-1108.

O'Connell, K.F., Maxwell, K.N., and White, J.G. (2000). The spd-2 gene is required for polarization of the anteroposterior axis and formation of the sperm asters in the Caenorhabditis elegans zygote. Developmental Biology 222, 55-70.

O'Donnell, L. (2014). Mechanisms of spermiogenesis and spermiation and how they are disturbed. Spermatogenesis 4, e979623.

Oegema, K., and Hyman, T. (2006). Cell Division (Pasadena (CA): WormBook; 2005-2018). 
Oswald, J., Engemann, S., Lane, N., Mayer, W., Olek, A., Fundele, R., Dean, W., Reik, W., and Walter, J. (2000). Active demethylation of the paternal genome in the mouse zygote. Current Biology 10, 475478.

Otto, S.P., and Lenormand, T. (2002). Resolving the paradox of sex and recombination. Nature Reviews Genetics 3, 252-261.

Panzica, M.T., and McNally, F.J. (2018). Mechanisms that prevent catastrophic interactions between paternal chromosomes and the oocyte meiotic spindle. Cell Cycle 17, 529-534.

Patterson, K., Molofsky, A.B., Robinson, C., Acosta, S., Cater, C., and Fischer, J.A. (2004). The Functions of Klarsicht and Nuclear Lamin in Developmentally Regulated Nuclear Migrations of Photoreceptor Cells in the Drosophila Eye. Molecular Biology of the Cell 15, 600-.

Payne, C., Rawe, V., Ramalho-Santos, J., Simerly, C., and Schatten, G. (2003). Preferentially localized dynein and perinuclear dynactin associate with nuclear pore complex proteins to mediate genomic union during mammalian fertilization. Journal of Cell Science 116, 4727-4738.

Pelham B, H.R. (1991). Multiple targets for brefeldin A . Cell 67:449-451, 449-451.

Pfender, S., Kuznetsov, V., Pleiser, S., Kerkhoff, E., and Schuh, M. (2011). Spire-Type Actin Nucleators Cooperate with Formin-2 to Drive Asymmetric Oocyte Division. Current Biology 21, 955-960.

Pfender, S., Kuznetsov, V., Pasternak, M., Tischer, T., Santhanam, B., and Schuh, M. (2015). Live imaging RNAi screen reveals genes essential for meiosis in mammalian oocytes. Nature 524, 239-242.

Politi, A.Z., Cai, Y., Walther, N., Hossain, M.J., Koch, B., Wachsmuth, M., and Ellenberg, J. (2018). Quantitative mapping of fluorescently tagged cellular proteins using FCS-calibrated four-dimensional imaging. Nature Protocols 13, 1445-1464.

Pollard, T.D. (2016). Actin and Actin-Binding Proteins. Cold Spring Harbor Perspectives in Biology.

Pylypenko, O., Welz, T., Tittel, J., Kollmar, M., Chardon, F., Malherbe, G., Weiss, S., Michel, C.I.L., Samol-Wolf, A., Grasskamp, A.T., et al. (2016). Coordinated recruitment of Spir actin nucleators and myosin $\mathrm{V}$ motors to Rab11 vesicle membranes. ELife 5, 1-25.

Quinlan, M.E., Heuser, J.E., Kerkhoff, E., and Mullins, R.D. (2005). Drosophila Spire is an actin nucleation factor. Nature 433, 382-388.

Rabut, G., and Ellenberg, J. (2004). Automatic real-time three-dimensional cell tracking by fluorescence microscopy. Journal of Microscopy 216, 131-137. 
Rauh, N.R., Schmidt, A., Bormann, J., Nigg, E.A., and Mayer, T.U. (2005). Calcium triggers exit from meiosis II by targeting the APC/C inhibitor XErp1 for degradation. Nature 437, 1048-1052.

Reichmann, J., Nijmeijer, B., Hossain, M.J., Eguren, M., Schneider, I., Politi, A.Z., Roberti, M.J., Hufnagel, L., Hiiragi, T., and Ellenberg, J. (2018). Dual-spindle formation in zygotes keeps parental genomes apart in early mammalian embryos. Science 361, 189-193.

Reiner, O., Carrozzo, R., Shen, Y., Wehnert, M., Faustinella, F., Dobyns, W.B., Caskey, C.T., and Ledbetter, D.H. (1993). Isolation of a Miller-Dicker lissencephaly gene containing G protein $\beta$-subunitlike repeats. Nature $364,717-721$.

Reinsch, S., and Gönczy, P. (1998). Mechanisms of nuclear positioning. Journal of Cell Science 111, 2283-2295.

Riggs, B., Rothwell, W., Mische, S., Hickson, G.R.X., Matheson, J., Hays, T.S., Gould, G.W., and Sullivan, W. (2003). Actin cytoskeleton remodeling during early Drosophila furrow formation requires recycling endosomal components Nuclear-fallout and Rab11. The Journal of Cell Biology 163, 143-154.

Robineau, S., Chabre, M., and Antonny, B. (2000). Binding site of brefeldin A at the interface between the small G protein ADP-ribosylation factor 1 (ARF1) and the nucleotide-exchange factor Sec7 domain. Proceedings of the National Academy of Sciences 97, 9913-9918.

Robinson, D.N., and Cooley, L. (2002). Genetic Analysis of the Actin Cytoskeleton in the Drosophila Ovary . Annual Review of Cell and Developmental Biology 13, 147-170.

Roland, J., Kenworthy, A.K., Peranen, J., Caplan, S., and Goldenring, J.R. (2007). Myosin Vb Interacts with Rab8a on a Tubular Network Containing EHD1 and EHD3. Molecular Biology of the Cell 18, 28382837.

Rougier, N., Bourc'his, D., Molina Gomes, D., Niveleau, A., Plachot, M., Pàldi, A., and Viegas-Péquignot, E. (1998). Chromosome methylation patterns during mammalian preimplantation development. Genes and Development 12, 2108-2113.

Rouvière, C., Houliston, E., Carré, D., Chang, P., and Sardet, C. (1994). Characteristics of pronuclear migration in Beroe ovata. Cell Motility and the Cytoskeleton 29, 301-311.

Ryley, D.A., Wu, H.H., Leader, B., Zimon, A., Reindollar, R.H., and Gray, M.R. (2005). Characterization and mutation analysis of the human FORMIN-2 (FMN2) gene in women with unexplained infertility. Fertility and Sterility 83, 1363-1371.

Saitoh, M., Ishikawa, T., Matsushima, S., Naka, M., and Hidaka, H. (1987). Selective inhibition of 
catalytic activity of smooth muscle myosin light chain kinase. The Journal of Biological Chemistry 262, 7796-7801.

Sakkas, D., Ramalingam, M., Garrido, N., and Barratt, C.L.R. (2015). Sperm selection in natural conception: What can we learn from Mother Nature to improve assisted reproduction outcomes? Human Reproduction Update 21, 711-726.

Sathananthan, A.H., Kola, I., Osborne, J., Trounson, A., Nga, S.C., Bongsot, A., and Ratnamt, S.S. (1991). Centrioles in the beginning of human development (sperm centriole/fertilization/syngamy/pronucleus/ultrastructure). Developmental Biology 88, 4806-4810.

Schaar, B.T., and McConnell, S.K. (2005). Cytoskeletal coordination during neuronal migration. Proceedings of the National Academy of Sciences 102, 13652-13657.

Schatten, G., and Schatten, H. (1981). Effects of Motility Inhibitors During Sea Urchin Fertilization. Experimental Cell Research 135, 311-330.

Schatten, G., Simerly, C., and Schatten, H. (1985). Microtubule configurations during fertilization, mitosis, and early development in the mouse and the requirement for egg microtubule-mediated motility during mammalian fertilization. Proceedings of the National Academy of Sciences 82, 41524156.

Schatten, G., Schatten, H., Spector, I., Cline, C., Paweletz, N., Simerly, C., and Petzelt, C. (1986). Latrunculin inhibits the microfilament-mediated processes during fertilization, cleavage and early development in sea urchins and mice. Experimental Cell Research 166, 191-208.

Schatten, H., Simerly, C., Maul, G., and Schatten, G. (1989). Microtubule assembly is required for the formation of the pronuclei, nuclear lamin acquisition, and DNA synthesis during mouse, but not sea urchin, fertillization. Gamete Research 23, 309-322.

Scheffler, K., Minnes, R., Fraisier, V., Paoletti, A., and Tran, P.T. (2015). Microtubule minus end motors kinesin-14 and dynein drive nuclear congression in parallel pathways. The Journal of Cell Biology 209, 47-58.

Schenk, J., Wilsch-Brauninger, M., Calegari, F., and Huttner, W.B. (2009). Myosin II is required for interkinetic nuclear migration of neural progenitors. Proceedings of the National Academy of Sciences $106,16487-16492$.

Schuh, M. (2013). An actin-dependent mechanism for long range vesicle transport. Nature Cell Biology $13,1431-1436$. 
Schuh, M., and Ellenberg, J. (2007). Self-Organization of MTOCs Replaces Centrosome Function during Acentrosomal Spindle Assembly in Live Mouse Oocytes. Cell 130, 484-498.

Schuh, M., and Ellenberg, J. (2008). A New Model for Asymmetric Spindle Positioning in Mouse Oocytes. Current Biology 1986-1992.

Schulz, K.N., and Harrison, M.M. (2019). Mechanisms regulating zygotic genome activation. Nature Reviews. Genetics 20, 221-234.

Schumacher, N., Borawski, J.M., Leberfinger, C.B., Gessler, M., and Kerkhoff, E. (2004). Overlapping expression pattern of the actin organizers Spir-1 and formin-2 in the developing mouse nervous system and the adult brain. Gene Expression Patterns 4, 249-255.

Sciaky, N., Presley, J., Smith, C., Zaal, K.J.M., Cole, N., Moreira, J.E., Terasaki, M., Siggia, E., and Lippincott-Schwartz, J. (1997). Golgi tubule traffic and the effects of brefeldin A visualized in living cells. The Journal of Cell Biology 139, 1137-1155.

Shelley, M.J. (2015). The Dynamics of Microtubule/Motor-Protein Assemblies in Biology and Physics. Annual Review of Fluid Mechanics 48, 487-506.

Shinohara, R., Thumkeo, D., Kamijo, H., Kaneko, N., Sawamoto, K., Watanabe, K., Takebayashi, H., Kiyonari, H., Ishizaki, T., Furuyashiki, T., et al. (2012). A role for mDia, a Rho-regulated actin nucleator, in tangential migration of interneuron precursors. Nature Neuroscience 15, 373-380.

Shoji, S., Yoshida, N., Amanai, M., Ohgishi, M., Fukui, T., Fujimoto, S., Nakano, Y., Kajikawa, E., and Perry, A.C.F. (2006). Mammalian Emi2 mediates cytostatic arrest and transduces the signal for meiotic exit via Cdc20. EMBO Journal 25, 834-845.

Silver, L.M. (1995). Reproductive Performance: Comparison of Inbred Strains. In Mouse Genetics, (Oxford: Oxford University Press), p. 62.

Simerly, C., Wu, G.-J., Zoran, S., Ord, T., Rawlins, R., Jones, J., Navara, C., Gerrity, M., Rinehart, J., Binor, Z., et al. (1995). The paternal inheritance of the centrosome, the cell's microtubule-organizing center, in humans, and the implications for infertility. Nature Medicine 1, 47-52.

Sjöblom, T., Jones, S., Wood, L.D., Parsons, D.W., Lin, J., Barber, T.D., Mandelker, D., Leary, R.J., Ptak, J., Silliman, N., et al. (2006). The Consensus Coding Sequences of Human Breast and Colorectal Cancers. Science 314, 268-274.

So, C., Seres, K.B., Steyer, A.M., Mönnich, E., Clift, D., Pejkovska, A., Möbius, W., and Schuh, M. (2019). A liquid-like spindle domain promotes acentrosomal spindle assembly in mammalian oocytes. Science 
364 , eaat9557.

Sobajima, T., Yoshimura, S. -i., Iwano, T., Kunii, M., Watanabe, M., Atik, N., Mushiake, S., Morii, E., Koyama, Y., Miyoshi, E., et al. (2015). Rab11a is required for apical protein localisation in the intestine. Biology Open 4, 86-94.

Spang, A., Herrmann, J.M., Hamamoto, S., and Schekman, R. (2001). The ADP Ribosylation FactorNucleotide Exchange Factors Gea1p and Gea2p Have Overlapping, but Not Redundant Functions in Retrograde Transport from the Golgi to the Endoplasmic Reticulum. Molecular Biology of the Cell 12, 1035-1045.

Spiller, C.M., and Bowles, J. (2015). Germ Cell Biology Sex determination in mammalian germ cells. Asian Journal of Andrology 17, 427-432.

Spradling, A.C. (1993). Developmental genetics of oogenesis. In The Development of Drosophila Melanogaster, M. Bate, and A. Martinez Arias, eds. (New York: Cold Spring Harbor Laboratory Press), pp. 1-70.

Stanly, T.A., Fritzsche, M., Banerji, S., Garc, E., Bernardino, J., Serna, D., Jackson, D.G., and Eggeling, C. (2016). Critical importance of appropriate fixation conditions for faithful imaging of receptor microclusters. Biology Open 5, 1343-1350.

Steinecke, A., Gampe, C., Nitzsche, F., and Bolz, J. (2014). DISC1 knockdown impairs the tangential migration of cortical interneurons by affecting the actin cytoskeleton. Frontiers in Cellular Neuroscience 8, 1-13.

Stenmark, H. (2009). Rab GTPases as coordinators of vesicle traffic. Nature Reviews Molecular Cell Biology 10, 513-525.

Stewart-Savage, J., and Grey, R.D. (1982). The Temporal and Spatial Relationships between Cortical Contraction, Sperm Trail Formation, and Pronuclear Migration in Fertilized Xenopus Eggs. Roux's Archives of Developmental Biology 191, 241-245.

Stricker, S.A. (1999). Comparative biology of calcium signaling during fertilization and egg activation in animals. Developmental Biology 211, 157-176.

Strome, S., and Wood, W.B. (1983). Generation of asymmetry and segregation of germ-line granules in early C. elegans embryos. Cell 35, 15-25.

Sun, S.C., Wang, Z.B., Xu, Y.N., Lee, S.E., Cui, X.S., and Kim, N.H. (2011). Arp2/3 complex regulates asymmetric division and cytokinesis in mouse oocytes. PLoS ONE 6. 
Swope, R.E., and Kropf, D.L. (1993). Pronuclear positioning and migration during fertilization in Pelvetia. Developmental Biology 157, 269-276.

Tanenbaum, M.E., Vale, R.D., and McKenney, R.J. (2013). Cytoplasmic dynein crosslinks and slides antiparallel microtubules using its two motor domains. ELife 2, 1-20.

Tanimoto, H., Kimura, A., and Minc, N. (2016). Shape-motion relationships of centering microtubule asters. The Journal of Cell Biology 212, 777-787.

Technau, M., and Roth, S. (2008). The Drosophila KASH domain proteins Msp-300 and Klarsicht and the SUN domain protein klaroid have no essential function during oogenesis. Fly 2, 82-91.

Tokuhiro, K., and Dean, J. (2018). Glycan-independent Gamete Recognition Triggers Egg Zinc Sparks and ZP2 Cleavage to Prevent Polyspermy. Developmental Cell 46, 627-640.

Tomlinson, A. (1985). The cellular dynamics of pattern formation in the eye of Drosophila. Journal of Embryology and Experimental Morphology 89, 313-331.

Torres-Padilla, M.E., Bannister, A.J., Hurd, P.J., Kouzarides, T., and Zernicka-Goetz, M. (2006). Dynamic distribution of the replacement histone variant H3.3 in the mouse oocyte and preimplantation embryos. International Journal of Developmental Biology 50, 455-461.

Tran, P.T., Marsh, L., Doye, V., Inoué, S., and Chang, F. (2001). A mechanism for nuclear positioning in fission yeast based on microtubule pushing. The Journal of Cell Biology 153, 397-411.

Tsafriri, A., Chun, S.Y., Zhang, R., Hsueh, A.J., and Conti, M. (1996). Oocyte maturation involves compartmentalization and opposing changes of cAMP levels in follicular somatic and germ cells: studies using selective phosphodiesterase inhibitors. Developmental Biology 178, 393-402.

Tsai, J.W., Chen, Y., Kriegstein, A.R., and Vallee, R.B. (2005). LIS1 RNA interference blocks neural stem cell division, morphogenesis, and motility at multiple stages. The Journal of Cell Biology 170, 935-945.

Tsai, J.W., Bremner, K.H., and Vallee, R.B. (2007). Dual subcellular roles for LIS1 and dynein in radial neuronal migration in live brain tissue. Nature Neuroscience 10, 970-979.

Ullrich, O., Reinsch, S., Urbé, S., Zerial, M., and Parton, R.G. (1996). Rab11 regulates recycling through the pericentriolar recycling endosome. The Journal of Cell Biology 135, 913-924.

Uraji, J., Scheffler, K., and Schuh, M. (2018). Functions of actin in mouse oocytes at a glance. Journal of Cell Science 131, jcs218099.

Vizcarra, C.L., Kreutz, B., Rodal, A.A., Toms, A. V., Lu, J., Zheng, W., Quinlan, M.E., and Eck, M.J. (2011). 
Structure and function of the interacting domains of Spire and Fmn-family formins. Proceedings of the National Academy of Sciences 108, 11884-11889.

Wang, B., Li, J., and Xiao, X. (2000a). Adeno-associated virus vector carrying human minidystrophin genes effectively ameliorates muscular dystrophy in $\mathrm{mdx}$ mouse model. Proceedings of the National Academy of Sciences 97, 13714-13719.

Wang, L., Wang, Z.B., Zhang, X., FitzHarris, G., Baltz, J.M., Sun, Q.Y., and Liu, X.J. (2008a). Brefeldin A disrupts asymmetric spindle positioning in mouse oocytes. Developmental Biology 313, 155-166.

Wang, X., Kumar, R., Navarre, J., Casanova, J.E., and Goldenring, J.R. (2000b). Regulation of vesicle trafficking in Madin-Darby canine kidney cells by Rab11a and Rab25. The Journal of Biological Chemistry 275, 29138-29146.

Wang, Z., Edwards, J.G., Riley, N., Provance, D.W.J., Karcher, R., Li, X., Davison, I., Ikebe, M., Mercer, J., Kauer, J., et al. (2008b). Myosin Vb Mobilizes Recycling Endosomes and AMPA Receptors for Postsynaptic Plasticity. PLoS ONE 135, 525-548.

von Wangenheim, D., Hauschild, R., Fendrych, M., Barone, V., Benková, E., and Friml, J. (2017). Live tracking of moving samples in confocal microscopy for vertically grown roots. ELife 6 .

Western, P.S., Miles, D.C., Van den Bergen, J.A., Burton, M., and Sinclair, A.H. (2008). Dynamic Regulation of Mitotic Arrest in Fetal Male Germ Cells. Stem Cells 26, 339-347.

Winder, S.J., Hemmings, L., Maciver, S.K., Bolton, S.J., Tinsley, J.M., Davies, K.E., Critchley, D.R., and Kendrick-Jones, J. (1995). Utrophin actin binding domain: analysis of actin binding and cellular targeting. Journal of Cell Science 108 ( Pt 1, 63-71.

Woychik, R.P., Stewart, T.A., Davis, L.G., D'Eustachio, P., and Leder, P. (1985). An inherited limb deformity created by insertional mutagenesis in a transgenic mouse. Nature 318, 36-40.

Woychik, R.P., Maas, R.L., Zeller, R., Vogt, T.F., and Leder, P. (1990). "Formins": proteins deduced from the alternative transcripts of the limb deformity gene. Nature $346,850-853$.

Wühr, M., Tan, E.S., Parker, S.K., Dietrich, W.H.I., and Mitchinson, T.J. (2010). A model for cleavage plane determination in early amphibian and fish embryos. Current Biology 20, 2040-2045.

Xiong, H., Mohler, W.A., and Soto, M.C. (2011). The branched actin nucleator Arp2/3 promotes nuclear migrations and cell polarity in the C. elegans zygote. Developmental Biology 357, 356-369.

Yi, K., Unruh, J.R., Deng, M., Slaughter, B.D., Rubinstein, B., and Li, R. (2011). Dynamic maintenance of 
asymmetric meiotic spindle position through Arp2/3-complex-driven cytoplasmic streaming in mouse oocytes. Nature Cell Biology 13, 1252-1258.

Yi, K., Rubinstein, B., Unruh, J.R., Guo, F., Slaughter, B.D., and Li, R. (2013). Sequential actin-based pushing forces drive meiosis I chromosome migration and symmetry breaking in oocytes. The Journal of Cell Biology 200, 567-576.

Yu, X.-J., Yi, Z., Gao, Z., Qin, D., Zhai, Y., Chen, X., Ou-Yang, Y., Wang, Z.-B., Zheng, P., Zhu, M.-S., et al. (2014). The subcortical maternal complex controls symmetric division of mouse zygotes by regulating F-actin dynamics. Nature Communications 5, 4887.

Zenker, J., White, M.D., Templin, R.M., Parton, R.G., Thorn-Seshold, O., Bissiere, S., and Plachta, N. (2017). A microtubule-organizing center directing intracellular transport in the early mouse embryo. Science 357, 925-928.

Zenker, J., White, M.D., Gasnier, M., Alvarez, Y.D., Lim, H.Y.G., Bissiere, S., Biro, M., and Plachta, N. (2018). Expanding Actin Rings Zipper the Mouse Embryo for Blastocyst Formation. Cell 173, 776791.e17.

Zeth, K., Pechlivanis, M., Samol, A., Pleiser, S., Vonrhein, C., and Kerkhoff, E. (2011). Molecular basis of actin nucleation factor cooperativity: Crystal structure of the Spir-1 kinase non-catalytic C-lobe domain (KIND).formin-2 formin Spir interaction motif (FSI) complex. The Journal of Biological Chemistry 286, 30732-30739.

Zhang, D., Pan, L., Yang, L.H., He, X.K., Huang, X.Y., and Sun, F.Z. (2005). Strontium promotes calcium oscillations in mouse meiotic oocytes and early embryos through InsP3 receptors, and requires activation of phospholipase and the synergistic action of InsP3. Human Reproduction 20, 3053-3061. Zhang, Q., Bethmann, C., Worth, N.F., Davies, J.D., Wasner, C., Feuer, A., Ragnauth, C.D., Yi, Q., Mellad, J.A., Warren, D.T., et al. (2007). Nesprin-1 and -2 are involved in the pathogenesis of Emery - Dreifuss muscular dystrophy and are critical for nuclear envelope integrity. Human Molecular Genetics 16, 2816-2833. 


\section{APPENDIX}

A

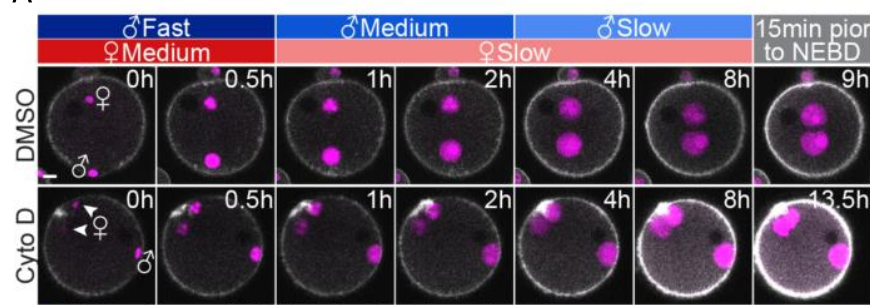

$\square$ H2B-mCherry (DNA) $\square$ MyrGFP (membrane)

D

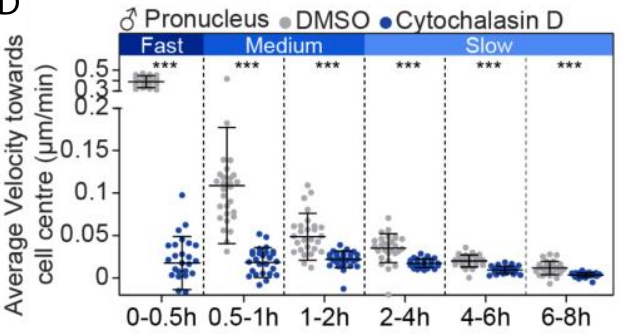

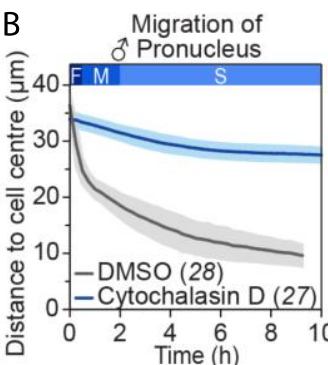

$\mathrm{E}$

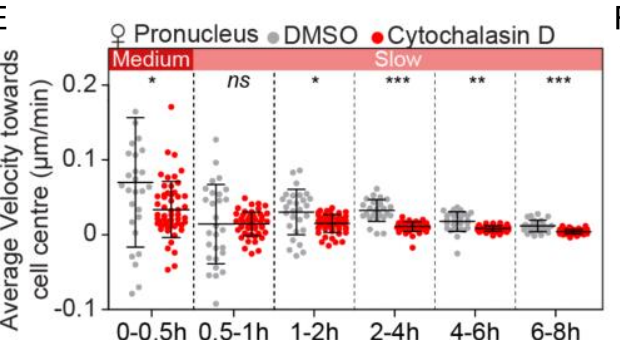

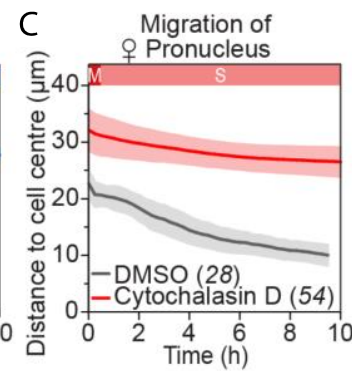

F

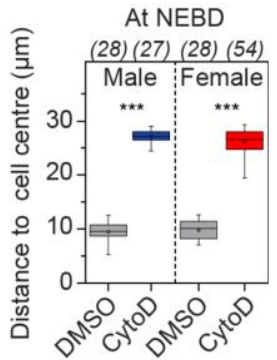

Figure 27: Cytochalasin D treatment completely perturbs pronuclear migration in mouse zygotes. (A) Three-dimensional time-lapse images of pronuclei (H2B-mCherry, magenta) and the cell surface (MyrGFP, white) in zygotes treated with DMSO or $5 \mu \mathrm{g} / \mathrm{mL}$ cytochalasin D. Female ( $(q)$ and male ( $\left.\sigma^{\prime}\right)$ genomes are shown at pronuclear formation (time-point Oh). Movement speeds are classified as in Fig. 1a. Scale bar, $10 \mu \mathrm{m}$. (B, C) The mean distance (thick line) of male ( $\left.\sigma^{\prime},(B)\right)$ and female ( $\left.\$,(C)\right)$ pronuclei to zygote centre during pronuclear migration in DMSO-or cytochalasin D-treated zygotes was calculated from data sets as shown in (A). Movement speeds are classified as in Figure 3. The total number of analysed zygotes specified in italics was pooled from three independent experiments. Standard Deviation shown as shaded areas. (D, E) Statistical plots of average velocities of male $(O,(D))$ and female $(q,(E))$ pronuclei during pronuclear migration in DMSO- or cytochalasin $D$ treated zygotes calculated from (o) and (p). Movement speeds are classified as in Figure 3; statistical plots as in Figure 3. Two-tailed student's t-test was used to test for significance. (F) Distance of pronuclei to zygote centre at NEBD in DMSO- or cytochalasin D-treated zygotes calculated from data sets in (A). All individual values (dots) are overlaid with a box plot. The box plot shows median (line), mean (small square), 5th, 95th (whiskers) and 25th and 75th percentile (boxes enclosing 50\% of the data) calculated from the total number of analysed zygotes specified in italics and pooled from four independent experiments. Two-tailed student's t-test was used to test for significance. Adapted from figure prepared for the unpublished manuscript; experiments and analysis done by Kathleen Scheffler and Julia Uraji. 
A
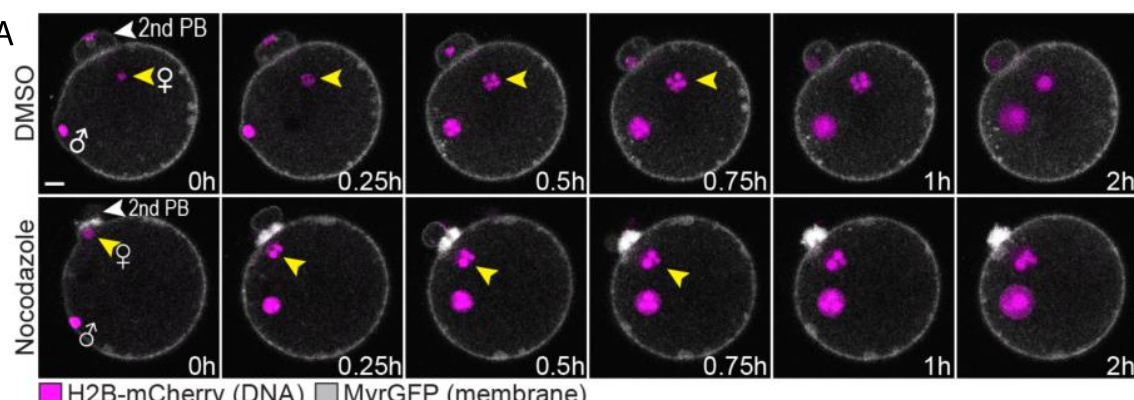

C

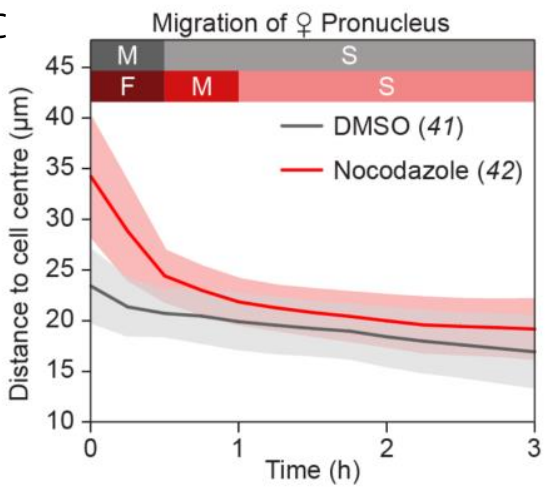

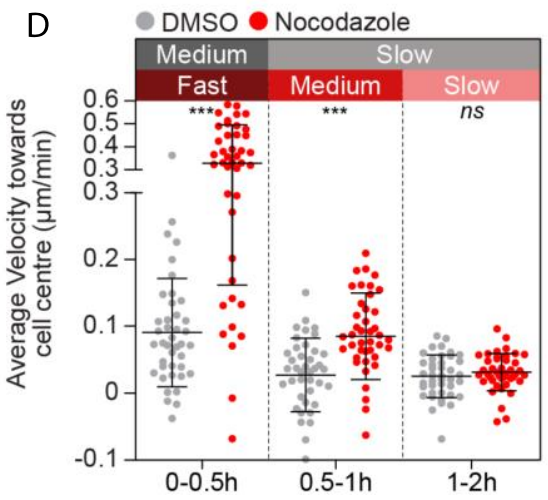

B At + pronuclear formation

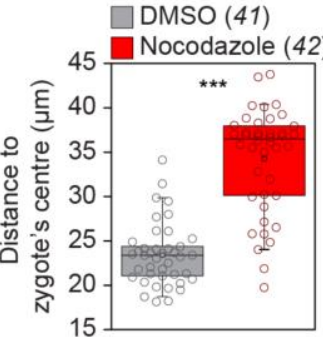

Figure 28: Nocodazole treatment can make the female pronucleus form closer to the cortex. (A) Three-dimensional timelapse images of pronuclei (H2B-mCherry, magenta) and the cell surface (MyrGFP, white) in zygotes treated with DMSO or $1 \mu \mathrm{M}$ nocodazole-treated from pronuclear formation (time-point Oh). Yellow arrows indicate the position of female ( $(\mathrm{)})$ pronuclei. Scale bar, $10 \mu \mathrm{m}$. (B) Distance of female pronuclei formation site from the cell centre in DMSO- or nocodazoletreated zygotes calculated from data sets as shown in (D). All individual values (dots) are overlaid with a box plot. The box plot shows median (line), mean (small square), 5th, 95th (whiskers) and 25th and 75th percentile (boxes enclosing 50\% of the data) calculated from the total number of analysed zygotes specified in italics and pooled from four independent experiments. Two-tailed student's t-test was used to test for significance. (C) The mean distance (black line) of female (f) pronuclei to zygote centre during pronuclear migration in DMSO- or nocodazole-treated zygotes was calculated from data sets as shown in (A). Movement speeds are classified as in Figure 3. Total number of analysed zygotes specified in italics was pooled from four independent experiments. Standard Deviation shown as shaded areas. (D) Statistical plots of average velocities of female (f) pronuclei during pronuclear migration in DMSO- or nocodazole-treated zygotes calculated from (C). Movement speeds and statistical plots are classified as in Figure 3. Two-tailed student's t-test was used to test for significance. Adapted from figure prepared for the unpublished manuscript; experiments and analysis done by Kathleen Scheffler. 


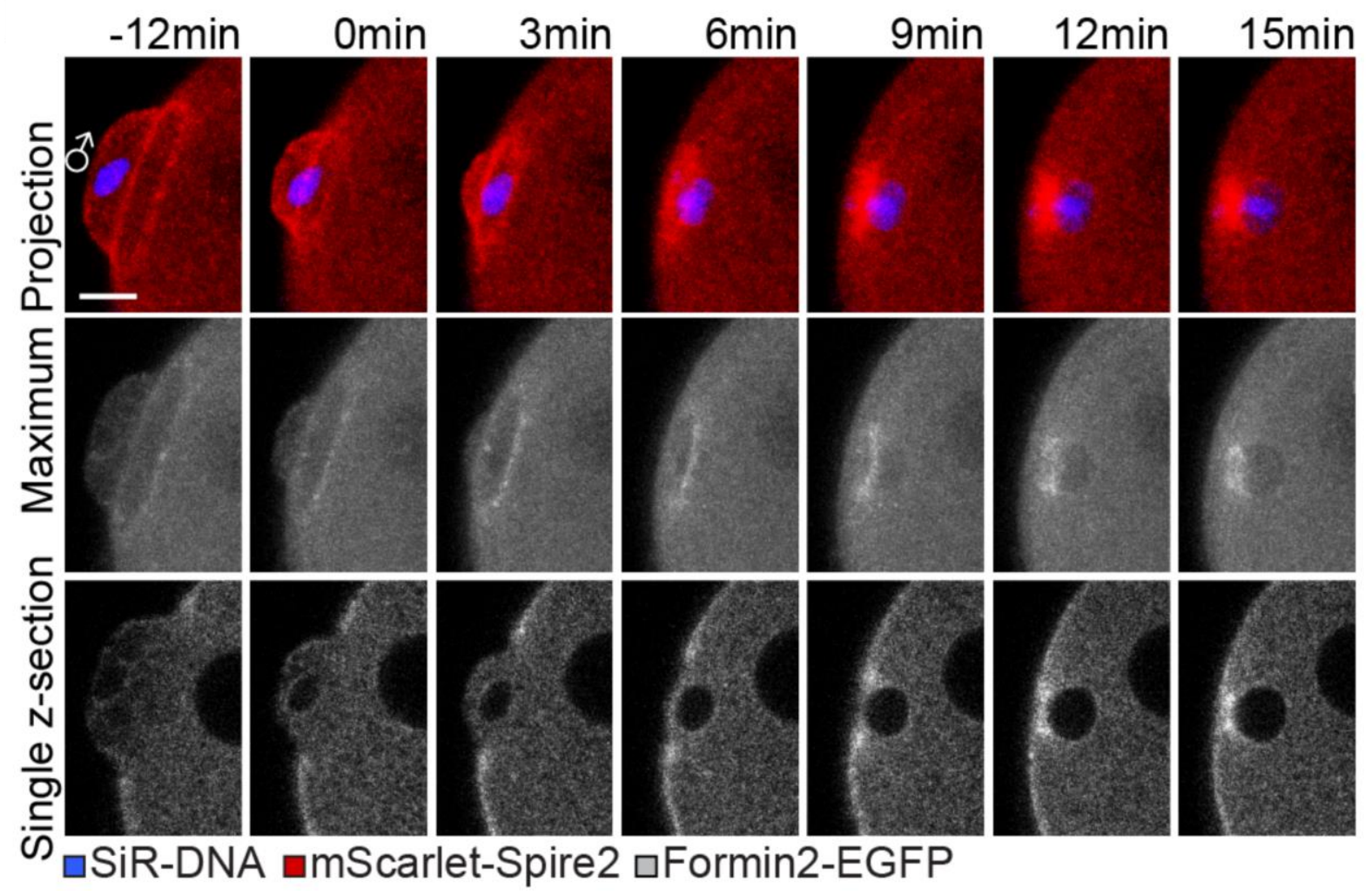

Figure 29: Co-expression of mScarlet-Spire2 and Formin-2-EGFP mRNA. Time-lapse images of mClover3-Spire2 (red), Formin-2-EGFP (white) and male pronucleus (SiR-DNA, blue) in live zygotes displayed as z-projection of 20 sections at $1.5 \mu \mathrm{m}$. Scale bar, $10 \mu \mathrm{m}$. Adapted from figure prepared for the unpublished manuscript; experiments and analysis done by Kathleen Scheffler.
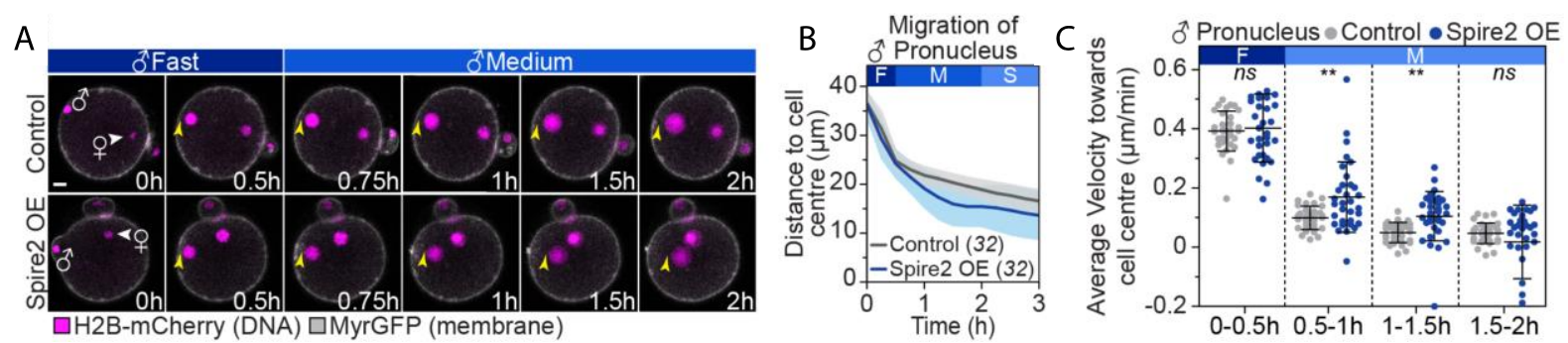

Figure 30: Fast phase of male pronuclear migration is elongated by SNAP-Spire2 Overexpression. (A) Confocal time-lapse images of pronuclei (H2B-mCherry, magenta) and the cell surface (MyrGFP, white) in zygotes expressing SNAP or SNAP-Spire2 mRNA. Female (f) and male ( $\left.\sigma^{\prime \prime}\right)$ genomes are shown at pronuclear formation (time-point Oh). Movement speeds are classified as in Fig. 1a. The yellow arrowheads indicate the gap between the male pronucleus and the cell surface. Scale bar, $10 \mu \mathrm{m}$. (B) The mean distance (thick line) of male $\left(O^{\prime \prime}\right)$ pronuclei to zygote centre during pronuclear migration in zygotes expressing SNAP or SNAP-Spire2 was calculated from data sets as shown in (A). Movement speeds are classified as in Figure 3. The total number of analysed zygotes specified in italics was pooled from three independent experiments. Standard deviation shown as shaded areas. (C) Statistical plots of average velocities of male ( $\left.\sigma^{7}\right)$ pronuclei during pronuclear migration in zygotes expressing SNAP or SNAP-Spire2 calculated from (B). Movement speeds and statistics are classified as in Figure 3. Two-tailed student's t-test was used to test for significance. Adapted from figure prepared for the unpublished manuscript; experiments and analysis done by Kathleen Scheffler. 